\title{
MELCOR Peer Review
}

mas? 1992 
Edited by Karyn Ames, Group IS-11

Composition by Gloria Mirabal, Group N-12

This work zuas supported by the US Nuclear Regulatory Commission, Office of Nuclear Regulatory Resenrch.

An Affirmutizu' Action/Equan Opiportunity Employer

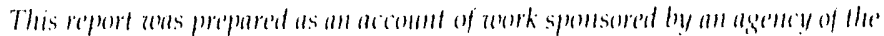

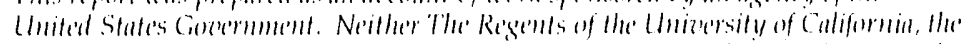

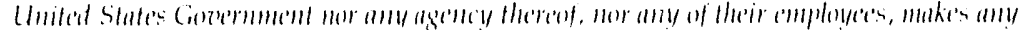

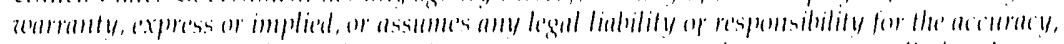

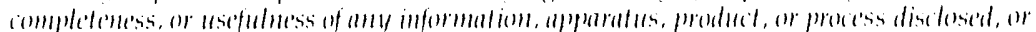

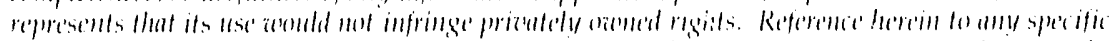

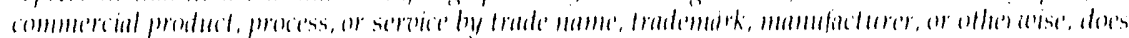

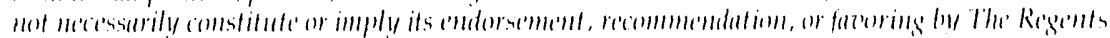

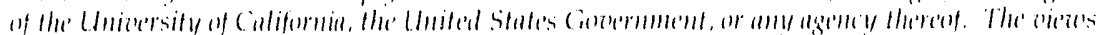

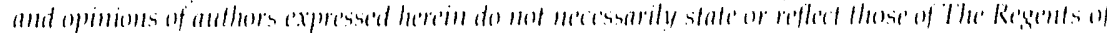

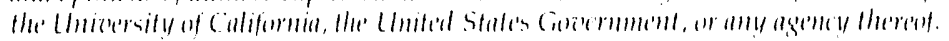




\title{
MELCOR Peer Review
}

\author{
B. E. Boyack \\ V. K. Dhir* \\ J. A. Gieseke** \\ T. J. Haste*** \\ M. A. Kenton \\ M. Khatib-Rahbar ${ }^{\dagger+}$ \\ M. T. Leonard ${ }^{\text {tt }}$ \\ R. Viskantr ${ }^{\dagger}$
}

*244522nd Stredt, Santa Momica CA 9(0405

*Ballu'lle, 505 King' Avemue, Columburs, OH 4.3207

***Winfrith Technology Center, Reactor Safe'y Studie's Departmemt, Dorchester, Dors't DT2 8DH, UNITFD KINGDOM

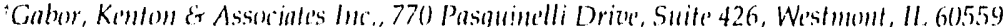

"Energy Re'searsh linc., P.O. B(ox 20134, Rockville, MD 20852

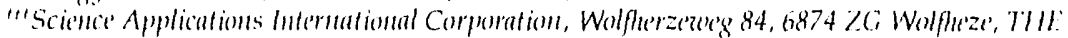
NETHIRLI.ANDS

"Purdur: Unierersity, Heat Transfer Laboratory, 1288 School of Mechanical Engintering. West Lafayetfle, IN 479017 


\section{CONTENTS}

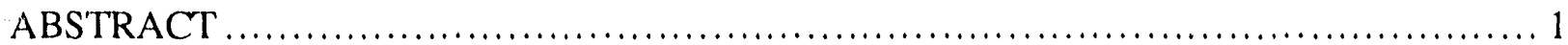

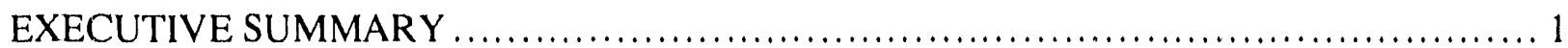

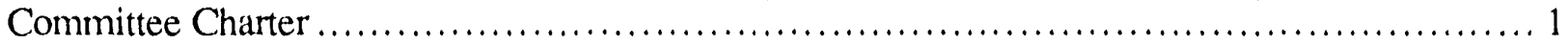

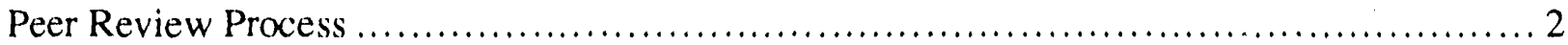

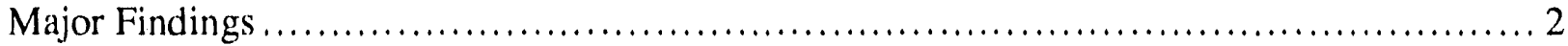

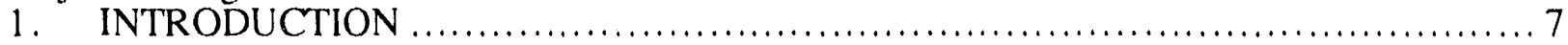

1.1. Committee Charter ..................................................................... 8

1.2. Committee Membership ............................................................... 8

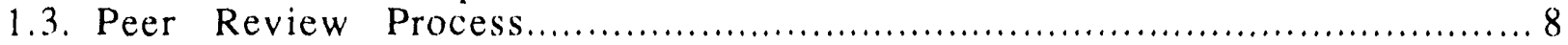

1.4. Presentation of Committee Findings ........................................................... 13

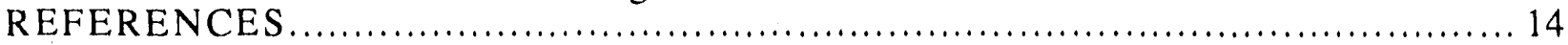

2. BOTTOM-UP REVIEW OF MELCOR PHENOMENOLOGICAL PACKAGES $\ldots \ldots \ldots \ldots 15$

2.1. $\mathrm{CVH} / \mathrm{FL}$ Phenomenological Package Review Summary ........................... 15

2.1.1. Phenomenological Package Description........................................ 15

2.1.2. Qualitative Perspective ............................................. 16

2.1.3. Technical Adequacy .............................................. 16

2.2. HS Phenomenological Package Review Summary ........................................ 17

2.2.1. Phenomenological Package Description.......................................... 17

2.2.2. Qualitative Perspective ................................................ 18

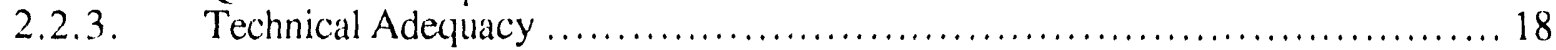

2.3. COR Phenomenological Package Review Summary ..................................... 18

2.3.1. Phenomenological Package Description........................................ 18

2.3.2. Qualitative Perspective ................................................. 19

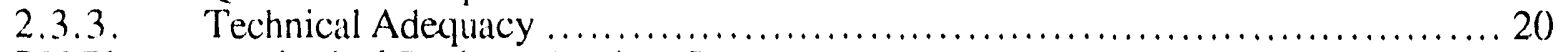

2.4. RN Phenomenological Package Review Summary .................................... 21

2.4.1. Phenomenological Package Description.............................................. 21

2.4.2. Qualitative Perspective ................................................... 21

$2.4 .3 \quad$ Technical Adequacy ................................................... 22

2.5. FDI Phenomenological Package Review Summary ................................... 23

2.5.1. Phenomenological Package Description......................................... 23

2.5.2. Qualitative Perspective .................................................... 23

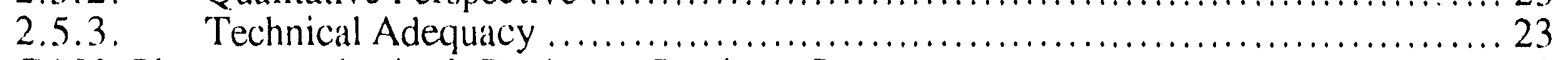

2.6. CAV Phenomenological Package Review Summary ........................................... 23

2.6.1. Phenomenological Package Description............................................ 23

2.6.2. Qualitative Perspective ............................................... 24

2.6.3. Technical Adequacy ........................................................... 24

2.7. BUR Phenomenological Package Review Summary .................................. 25

2.7.1. Phenomenological Package Description............................................. 25

2.7.2. Qualitative Perspective ................................................ 25

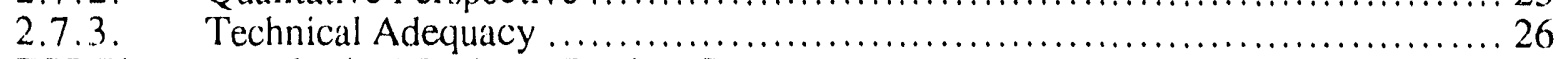

2.8. ESF Phenomenological Package Review Summary ............................................. 26

2.8.1. Phenomenological Package Description................................................. 26

2.8.2. Qualitative Perspective .......................................................... 27

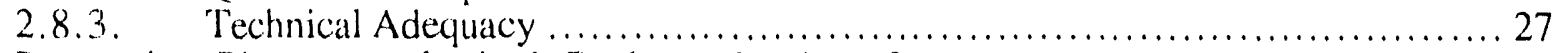

2.9. Properties Phenomenological Package Review Summary ....................................... 27

2.9.1. Phenomenological Package Description......................................... 27

2.9.2. Qualitative Perspective ........................................................ 28

2.9.3. Technical Adequacy .............................................................. 29

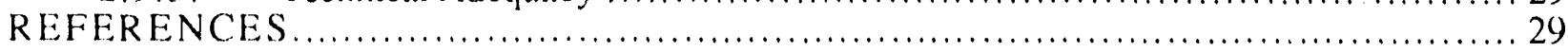


3. FINDINGS RELATIVE TO CODE OBJECTIVES .......................................... 31

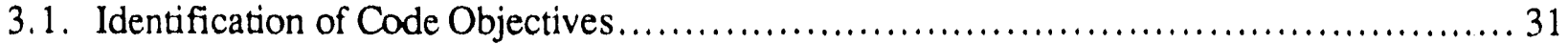

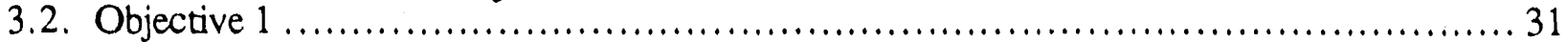

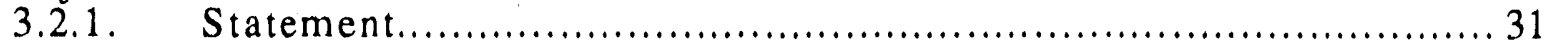

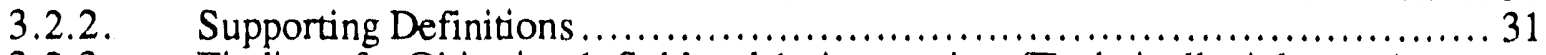

3.2.3. Findings for Objective 1, Sublevel 1: Appropriate (Technically Adequate)

Modeling of Phenomena Essential to the Description of Severe LWR

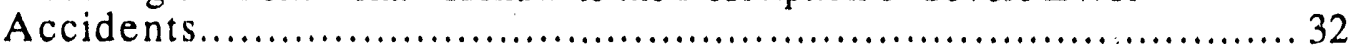

3.2.3.1. Deficient Models for Which Correction is Very Important ...................... 33

3.2.3.2. Deficient Models for Which Correction is Important ...................... 33

3.2.3.3. Deficient Models for Which Correction is Less Important ................. 33

3.2.4. Findings for Objective 1, Sublevel 2: Consistency Between Models and the Phenomenological Packages ....................................... 34

3.2.5. Findings for Objective 1, Sublevel 3: Code Capability to Represent the Plant Components, Systems, and Operator Action Features ...................... 34

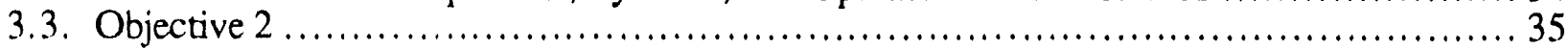

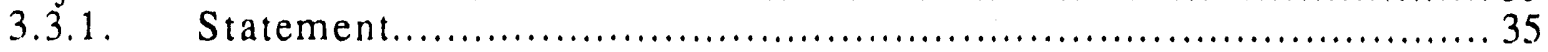

3.3.2. Supporting Definitions ............................................................. 35

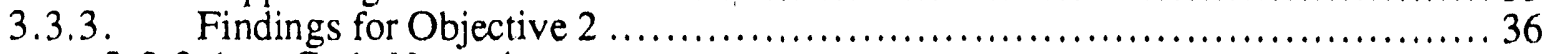

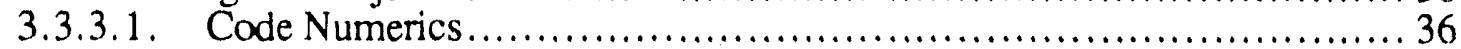

3.3.3.2. Comparison to Integral Benchmarks................................................ 37

3.3.3.3. Code-to-Code Comparisons ............................................ 41

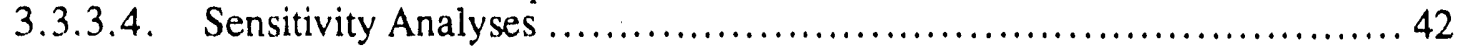

3.3.3.5. Committee Observations ..................................................... 44

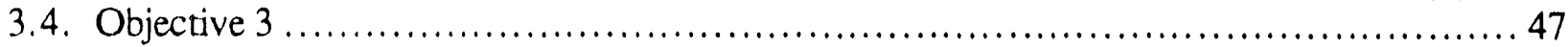

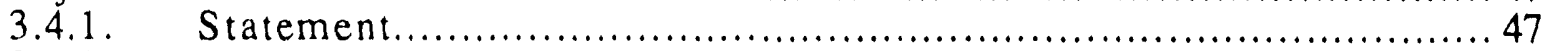

3.4.2. Supporting Definitions ........................................................ 47

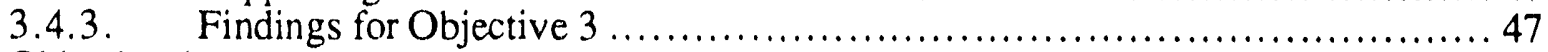

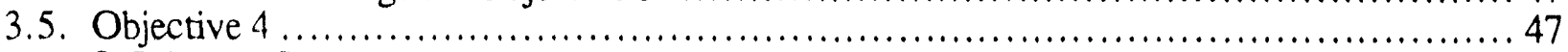

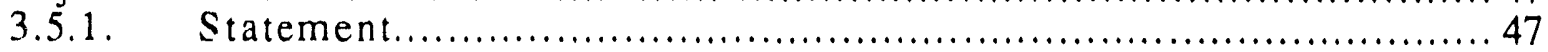

3.5.2. Supporting Definitions ..................................................... 47

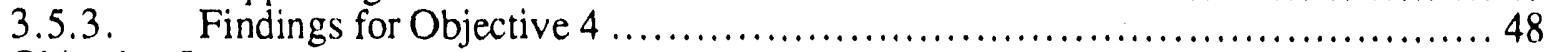

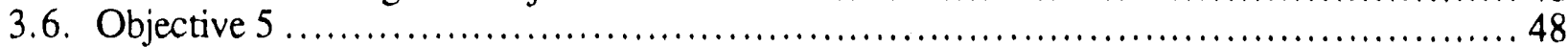

3.6.1. Statement.................................................................... 48

3.6.2. Supporting Definitions .................................................. 48

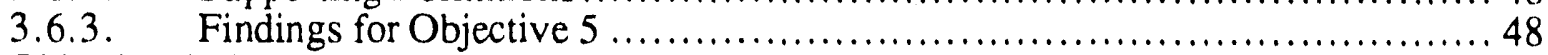

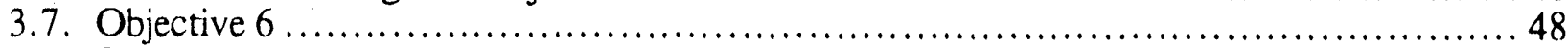

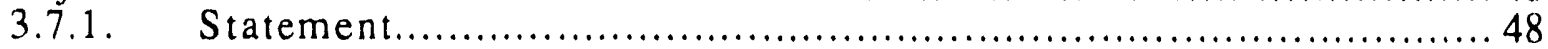

3.7.2. Supporting Definitions ................................................. 48

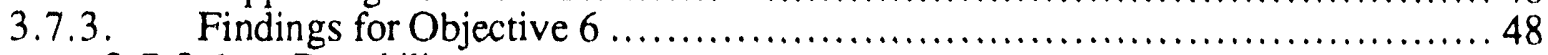

3.7.3.1. Portability ............................................................... 48

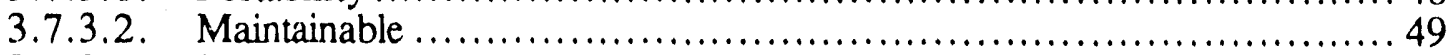

3.7.3.3. Structure that Facilitates Incorporation of New or

Alternative Phenomenological Models....................................... 49

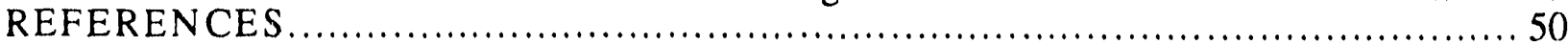

4. FINDINGS RELATIVE TO TARGETED APPLICATIONS …........................ 53

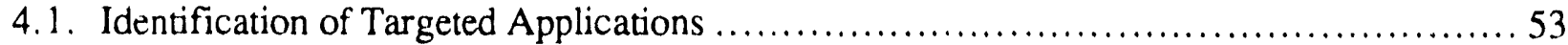

4.2. Screening for Missing Phenomenological Models .......................................... 53

4.3. Missing Models Necessary for PRA and AM Applications ......................... 54

4.4. Missing Models Desirable for PRA and Parametric AM Applications ........................ 56

4.5. Missing Models for Mechanistic AM Applications .................................... 57 
4.6. Conclusions Regarding the Completeness of MELCOR's Modeling

Vis-a-Vis the Targeted Applications...................................................... 59

REFERENCES ....................................................................... 59

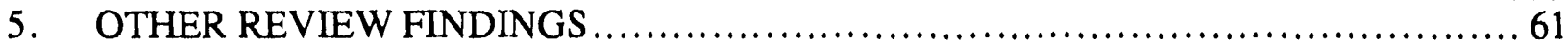

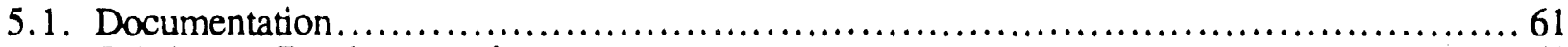

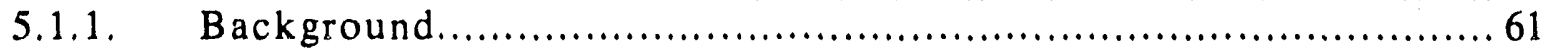

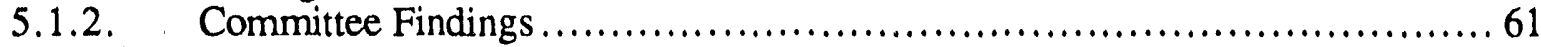

5.2. Inconsistency in Level of Modeling Detail ........................................ 63

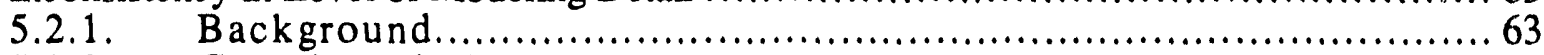

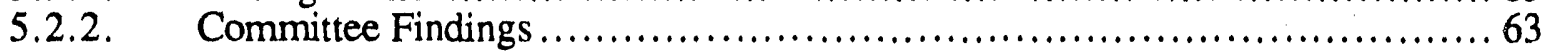

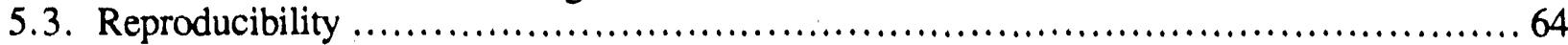

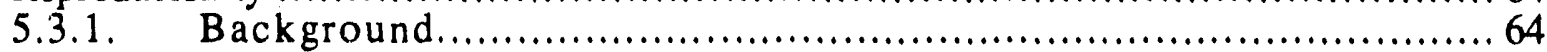

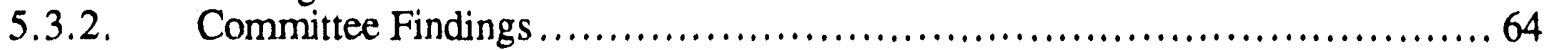

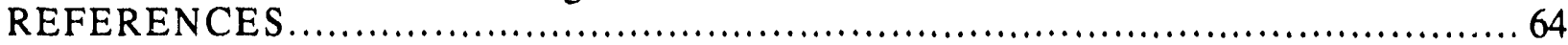

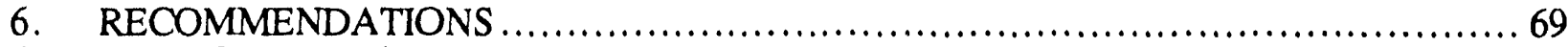

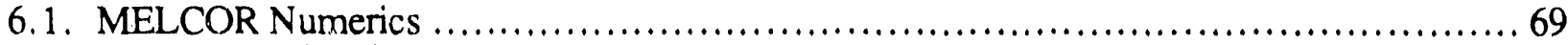

6.2. Models Missing from MELCOR Version 1.8.1 ..................................... 69

6.3. Existing MELCOR Models Needing Revision.............................................. 70

6.4. Need for Expanded MELCOR Assessment .......................................... 71

6.5. Documentation...................................................................... 71

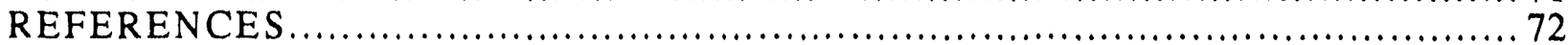

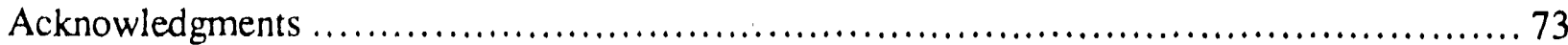

APPENDIX A Members of the MELCOR Peer Review Committee.............................. A-1

APPENDIX B MELCOR Document Data Base ........................................ B-1

APPENDIX C Severe Accident Phenomena ............................................. C.1

APPENDIX D Hydrodynamics Behavior (CVH, FL) Packages.......................... D-1

ANNEX D-1 MELCOR Input for Simulation of GE Vessel

ANNEX Dlowdown Tests...................................... D-47

ANNEX D-2 MELCOR Input for Simulation of a Simple

Condensation Problem................................. D-51

APPENDIX E Heat Structure (HS) Package............................................. E-1

APPENDIX F Core Heatup and Degradation (COR) Package .............................. F-1

APPENDIX G Radionuclide (RN) Package .............................................. G-1

APPENDIX H Core-Concrete Interaction (CAV) Package $\ldots \ldots \ldots \ldots \ldots \ldots \ldots \ldots \ldots \ldots \ldots \ldots . .1$

APPENDIX I Gas Combustion (BUR) Package.......................................... I-1

APPENDIX J Engineered Safety Features (ESF) Package . ............................ J-1

APPENDIX K Properties Packages ................................................. K-1

APPENDIX L Other Models Missing From MELCOR $\ldots \ldots \ldots \ldots \ldots \ldots \ldots \ldots \ldots \ldots \ldots \ldots \ldots$ L 1 


\section{TABLES}

1-I MELCOR Bottom-Up Review Classification of Findings ........................ 13

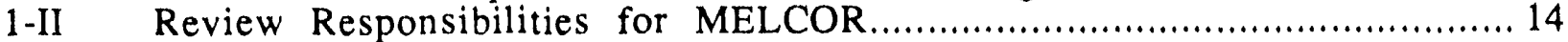

D-I Summary of CVH/FL Parameter and Sensitivity Studies.......................... D-6

D-Ila Summary of Review Findings (Initial Transient, Depletion, and Heatup Interval) .......................................................... 38

D-IIb Summary of Detailed Findings (Core Uncovery Interval) ........................ D-39

D-IIc Summary of Review Findings (Melt Relocation, Slump,

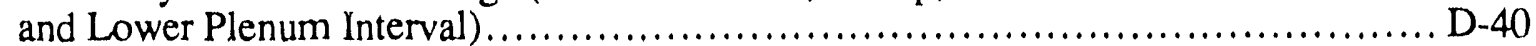

D-IId Summary of Review Findings (Post-Vessel Failure Interval) ...................... D-41

D-IIIa Summary of Hydrodynamics Model and Package Adequacy ........................ D-41

D-IIIb Summary of Hydrodynamics Model and Package Adequacy (Cont.) ................. D-42

D-IIIc Summary of Hydrodynamics Model and Package Adequacy (Cont.)................. D-43

D-IV Prioritization of Review Findings for CVH/FL Packages .......................... D-44

E-I Summary of Phenomenological Models in HS Package .................................. E-3

F-I Summary of Phenomenological Models in COR Package and Model Adequacy .............................................................. F-

H-I Summary of Phenomenological Models in the CAV Package and

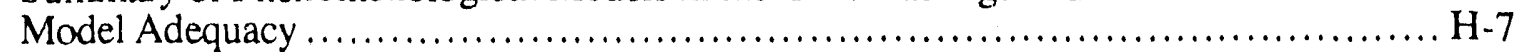

H-II Prioritization of Review Findings for the CAV Package ......................... H-8

K-I Summary of Noncondensable Gas and Water Property Package Model and Package Accuracy...................................................................... K-3

K-II Summary of Material Property Model and Package Accuracy ............................ K-4

K-III Summary of Decay Heat Package Model and Package Accuracy ..................... K-5

\section{FIGURES}

D-1. Schematic of GE large blowdown vessel .................................... D-8

D-2. Comparison of MELCOR pressure predictions and GE Test 5801-13 experimental data (choking factor of 1.0; maximum time step of $2.0 \mathrm{~s}$ ) .......................... D-9

D-3. Comparison of MELCOR pressure predictions and GE Test 5801-13 (choking factor of 0.70 ; maximum time step of $2.0 \mathrm{~s}$ ) ........................... D-10

D-4. CPU time vs real time (choking factor of 1.0; maximum time step of $2.0 \mathrm{~s}$ ) ............ D-11

D-5. Comparison of MELCOR pressure predictions for (a) one-flow path, and (b) two-flow path link (GE Test 5801-13; choking factor of 1.0; maximum time step of $2.0 \mathrm{~s}$ )....... D-12

D-6. Comparison of MELCOR pressure predictions and GE Test 5803-1 experimental data (choking factor of 1.0; maximum time step of $2 \mathrm{~s}$ ) .......................... D-14

D-7. Condensation problem schematic ............................................... 15

D-8. Void fraction vs time (equilibrium thermodynamics; $1-\mathrm{m} / \mathrm{s}$ fill) ..................... D-16

D-9. Pressure vs time (equilibrium thermodynamics; $1-\mathrm{m} / \mathrm{s}$ fill) ........................... D-17

D-10. Vapor temperature vs time (equilibrium thermodynamics; $1-\mathrm{m} / \mathrm{s}$ fill) .................... D-18

D-11. Liquid temperature vs time (equilibrium thermodynamics; $1-\mathrm{m} / \mathrm{s}$ fill) ............... D-19

D-12. Vapor velocity vs time (equilibrium thermodynamics; $1-\mathrm{m} / \mathrm{s}$ fill) ................... D-20)

D-13. Liquid velocity vs time (equilibrium thermodynamics; $1-\mathrm{m} / \mathrm{s}$ fill) ....................... D-21

D-14. Void fraction vs time (nonequilibrium thermodynamics; $1-\mathrm{m} / \mathrm{s}$ fill) $\ldots \ldots \ldots \ldots \ldots \ldots \ldots$. D-22

D-15. Pressure vs time (nonequilibrium thermodynamics; $1-\mathrm{m} / \mathrm{s}$ fill)........................ D-23

D-16. Vapor temperature vs time (nonequilibrium thermodynamics; $1-\mathrm{m} / \mathrm{s}$ fill) $\ldots \ldots \ldots \ldots \ldots \ldots$ D-24

D-17. Liquid temperature vs time (nonequilibrium thermodynamics; $1-\mathrm{m} / \mathrm{s}$ fill)................ D-25

D-18. Vapor velocity vs time (nonequilibrium thermodynamics; $1-\mathrm{m} / \mathrm{s}$ fill) .................... D-26 
D-19. Liquid velocity vs time (nonequilibrium thermodynamics; $1-\mathrm{m} / \mathrm{s}$ fill) $\ldots \ldots \ldots \ldots \ldots \ldots \ldots$ D-27

D-20. Condensation problem schematic .......................................... D-29

D-21. Air-water closed loop-void fraction vs time (cell 10) ............................... D-30

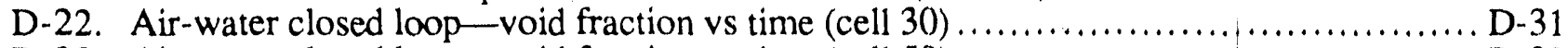

D-23. Air-water closed loop-void fraction vs time (cell 50) ........................... D-32

D-24. Air-water closed loop-void fraction vs time (cell 60) ............................. D-33

D-25. Air-water closed loop-void fraction vs time (cell 80) .......................... D-34

D-26. Air-water closed loop-void fraction vs time (cell 100) .............................. D-35 


\title{
MELCOR PEER REVIEW
}

\author{
by \\ B. E. Boyack, V. K. Dhir, J. A. Gieseke, T. J. Haste, M. A. Kenton, \\ M. Khatib-Rahbar, M. T. Leonard, and R. Viskanta
}

\begin{abstract}
MELCOR is a fully integrated, engineering-level computer code that models the progression of severe accidents in light water reactor nuclear power plants. The newest version of MELCOR is Version 1.8.1, July 1991. MELCOR development has reached the point that the United States Nuclear Regulatory Commission sponsored a broad technical review by recognized experts to determine or confirm the technical adequacy of the code for the serious and complex analyses it is expected to perform. For this purpose, an eight-member MELCOR Peer Review Committee was organized. The Committee has completed its review of the MELCOR code; the review process and findings of the MELCOR Peer Review Committee are documented in this report. The Committee has determined that recommendations in five areas are appropriate: (1) MELCOR numerics, (2) models missing from MELCOR Version 1.8.1, (3) existing MELCOR models needing revision, (4) the need for expanded MELCOR assessment, and (5) documentation.
\end{abstract}

\section{EXECUTIVE SUMMARY}

MELCOR is a fully integrated, engineering-level computer code that models the progression of severe accidents in light water reactor nuclear power plants. MELCOR is being developed at Sandia National Laboratories (SNL) for the United States Nuclear Regulatory Commission (USNRC) as a second-generation plant risk assessment tool and as the successor to the Source Term Code Package.

MELCOR has been under development since 1982. The newest version of MELCOR is Version 1.8.1, July 1991. The code has now reached sufficient maturity that a number of organizations inside and outside the NRC are using or are planning to use the code. Although quality control and valiuation efforts are in progress, there is a need to have a broad technical review by recognized experts to determine or confirm the technical adequacy of the code for the serious and complex analyses it is expected to perform. A peer review committee has been organized using recognized experts from the national laboratories, universities, MELCOR user community, and independent contractors to perform this assessment.

The objective of this report is to document the findings of the MELCOR I、 Review Committee that was formed to fulfill the charter described in the following section.

\section{Committee Charter}

The charter of the MELCOR Peer Review Committee was to (1) provide an independent assessment of the MELCOR code through a peer review process, (2) determine the technical adequacy of MELCOR for the complex analyses it is expected to perform, and (3) issue a final report describing the technical findings of the Committee. 


\section{Peer Review Process}

The Committee developed and followed a multistep process for the MELCOR Peer Review. The steps in the process are as follows:

1. Identify design objectives for the MELCOR code.

2. Identify targeted applications for the MELCOR code.

3. Identify the MELCOR code version to be reviewed.

4. Identify and distribute the MELCOR Document Data Base to Committee members.

5. Select plants and severe accident scenarios.

6. Develop a common Committee perspective regarding technical adequacy

7. Identify dominant phenomena for the plants and scenarios.

8. Define a "Standard of Technical Adequacy" to be used in developing findings.

9. Define a process for reviewing for technical adequacy.

10. Assess technical adequacy of individual models and/or correlations within the MELCOR phenomenological packages (bottom-up review).

11. Assess technical adequacy of the integral code against the MELCOR design objective and the MELCOR-targeted applications (top-down review).

12. Document findings in a summary report. Introduction.

Detailed descriptions of each step in the review process are provided in Chapter 1,

\section{Major Findings}

\section{Perspectives}

The NRC identified both the design objectives and targeted applications for the MELCOR code for the MELCOR Peer Review Committee. After defining a standard of technical adequacy and a process for evaluating technical adequacy, the Committee conducted a thorough review from two perspectives. The Committee first reviewed the individual models and correlations in each MELCOR phenomenological package or major code subdivision (bottom-up review); this resulted in the development of numerous findings. The Committee next reviewed the integral performance of the total code, leading to additional findings (top-down review). The MELCOR Peer Review Committee recognizes that resources for MELCOR development, revision, and enhancement are, and will continue to be, limited. Therefore, the Committee made a concerted effort to prioritize its findings. Those findings presented by way of the Committee recommendations (Chapter 6) are believed to be the minimum set of efforts that will permit MELCOR to fulfill its design objectives and effectively function for its targeted applications.

In developing its recommendations, the Committee, at the direction of the NRC, assigned primary importance to the adequacy of MELCOR for use in probabilistic risk assessment (PRA)targeted applications and considered mechanistic accident management (AM) studies to be of secondary importance for MELCOR application. The Committee screened each identified code deficiency by considering the importance of the deficiency relative to the potential impact on (1) the time of containment failure and (2) the magnitude of fission product release to the environment.

Finally, the Committee concluded that the technical requirements, when satisfied, will result in a technically adequate MELCOR for PRA applications, although the requirements may not always be sufficient for some parametric AM studies. If at a future time the role of the MELCOR code is expanded to include detailed AM studies focusing on timing and the magnitudes of key phenomena, Committee recommendations for needed improvements have been provided in the body of the report. 


\section{Degree of Completion}

The Committee determined that MELCOR is not a completed code and that additional development, as discussed below, is needed before MELCOR can reasonably satisfy its design objectives and be applied with confidence to its targeted applications. Completion of MELCOR can be measured in several ways. First, code completion can be measured relative to the existence of the needed models for all dominant phenomena that are to be predicted. Second, code completion can be measured relative to existence of the documentation needed to understand, appropriately apply, and interpret the code. Third, code completion can be measured relative to its demonstrated technical adequacy. The Committee determined that by each of these measures, further effort will be required before MELCOR can be considered to be complete.

Having made this point, the Committee also finds that considerable progress has been made in developing the MELCOR code. The component parts of MELCOR have been developed and assembled such that integrated calculations of some severe accident sequences in both boiling and pressurized water reactors (BWRs and PWRs) can be completed. Limited benchmarks have been prepared for some of the individual models and correlations and a limited set of benchmarks have been completed for the integrated code. An extensive set of documentation has been prepared, including a code manual, reference manuals for the phenomenological packages, and users' guides.

\section{Recommendations}

The Committee has determined that recommendations in five areas are appropriate: (1) MELCOR numerics, (2) models missing from MELCOR Version 1.8.1, (3) existing MELCOR models needins revision, (4) need for expanded MELCOR assessment, and (5) documentation.

MELCOR Numerics. The Committee concluded that code numerics are the source of a primary concern regarding the technical adequacy of the code. During the course of the MELCOR Peer Review, the results of several time-step sensitivity studies were made available to the Committee. These studies indicated that convergence to limiting values is not currently guaranteed as the time step decreases. In fact, key quantities vary erratically as the time step is changed. This severely compromises an analyst's ability to distinguish differences in calculated results due to physical processes from differences resulting from numerical chaos. The Committee has concluded that an improved understanding of the time-step sensitivities is important, that other input-parameter and modeling sensitivities can be expected, and that correction of the MELCOR numerics problems should be considered to be a high-priority activity.

Models Missing from MELCOR Version 1.8.1. The Committee concludes that models for the following phenomena, not currently modeled, should be given the highest priority for incorporation in MELCOR:

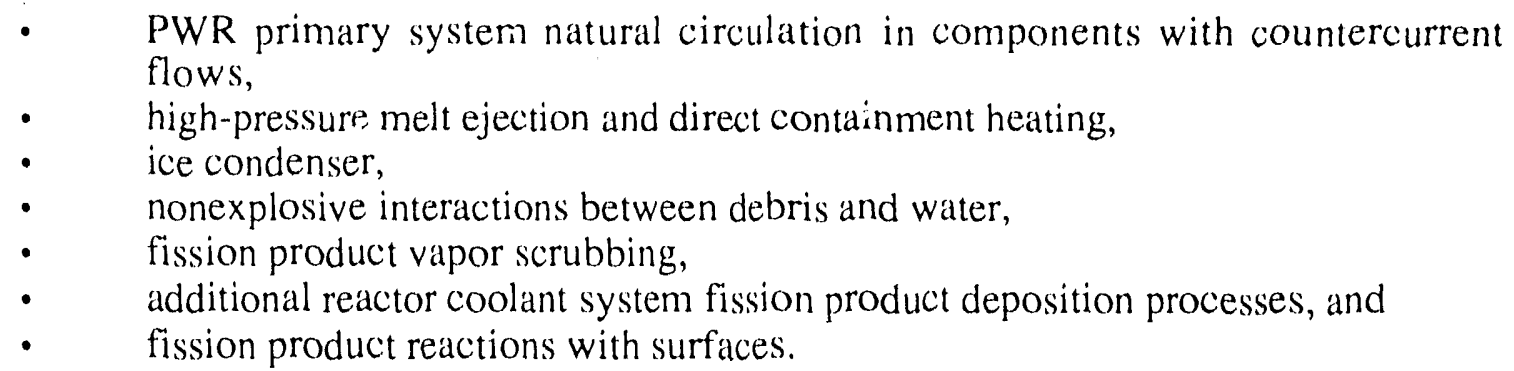

The Committee notes that funded model development activities are currently either planned or under way for either part or all of the PWR primary system natural circulation model, the highpressure melt ejection and direct containment heating model, and the ice condenser model. 
Existing MELCOR Models Needing Revision. The Committee's bottom-up review of the MELCOR phenomenological packages identified individual models that were of concern. The Committee completed a screening activity to determine which models should receive priority attention using as screening criteria (1) the time of containment failure and (2) the magnitude of the source term. The Committee recommends that the following issues, ranked by the Committee as "very important," be given the highest priority.

- An evaluation should be made to determine whether the water condensation/evaporation model used in the Hydrodynamic Behavior (CVH) package is implemented adequately as it supplies model information to the Radionuclide ( $R N$ ) package. Currently, condensation is treated independently in the CVH package from the calculations of aerosol particle growth and deposition in the RN package. The Committee feels that separating condensation growth from other growth calculations is a questionable procedure and the validity of this approach should be demonstrated by comparison with more exact models or data.

- Inconsistencies in treatment of chemical reactions between CORCON and VANESA should be resolved, and improvements should be made to the CORCON/MOD2 phase diagrams. Consolidation of CORCON and VANESA into a unified code as currently planned for CORCON/MOD3 is desirable. The Committee did not review CORCON/MOD3 models; however, based on a brief presentation to the Committee by SNL, it appears this code has the potential for remedying most of these concerns. A peer review of CORCON/MOD3 models would be desirable before its implementation into MELCOR.

- The model for condensation in containment (mass transfer) should be revised. With the existing model, steam condensation rates, predicted for conditions when the thermal resistance of the structure on which condensation occurs does not dominate, will be in serious error. The model used for condensation in the presence of noncondensables is seriously flawed.

- The pool scrubbing model is largely derived from previously available models but assumes spherical bubble shapes while correcting for bubble flattening by using coefficients derived from comparisons to other models. Impaction of particles from steam/air jets entering the pool would be expected to provide significant deposition for larger particles, but the effect is ignored in the current model. Decontamination factors computed with the current model are quite low in comparison with other models and the existing data base.

Need for Expanded MELCOR Assessment. The Committee concluded that the ability of MELCOR to calculate severe accident phenomena is not sufficiently demonstrated. Such a demonstration would be based on a documented collection of (1) sensitivity studies, (2) benchmarking activities using experimental data, and (3) code-to-code assessments.

The Committee has concluded that review of a comprehensive set of well-defined and executed sensitivity analyses is an important and necessary component of the effort to determine technical adequacy. Unfortunately, this comprehensive set of MELCOR sensitivity analyses does not presently exist. Rather, a sparse set of sensitivity studies exists.

By any measure, the MELCOR integral benchmarking effort is very small. The Committee has concluded that a more comprehensive and ongoing integral assessment program is needed. While encouraging an increasing pace for the integral assessment effort, the Committee emphasizes the importance of maintaining an overall perspective about the need to benchmark the individual models by using test data. A complete assessment of the technical adequacy of an integral code considers both the component parts and the adequacy of the integrated coded package. The review of the component parts focuses on the pedigree, applicability, and fidelity of the individual models and correlations. The Committee emphasizes the importance of having technically adequate detailed models and correlations which are, in reality, the building blocks of the integral code. 
Although code-to-code comparisons cannot and should not displace or replace code benchmarking efforts against test data, the Committee concludes that code-to-code comparisons can provide useful supplementary insights. For severe accident phenomena, their value lies not in the absolute, i.e., what is right and what is wrong, but in focusing attention on what is different. The Committee recommends that comparison activities continue in the future.

Documentation. The availability, content, completeness, and qualitv of documentation is an important factor influencing the outcome of a code peer review activity. The NRC has recently prepared and issued documentation guidance to organizations involved in the development of software for the NRC's Office of Nuclear Regulatory Research. Each of the documentation elements identified by the NRC exist in one form of MELCOR documentation or another. The body of existing documentation represents a significant and positive accomplishment. The Committee was able, for example, to accomplish a significant portion of its review using the dccumentation that has already been prepared. The Committee does, however, have concerns about particular aspects of the documentation and these are detailed in the following paragraphs.

The MELCOR summary report and phenomenological package reference manual cover the elements of the code or theory manual. The Committee determined, however, that the level of detail was less than that needed by the user community. After extensive interactions with the SNL staff, the Committee felt that the detailed descriptions of the models and correlations were lacking in some cases. That is, what was modeled was described but the descriptions of pedigree, applicability, and benchmarking were either inadequate or missing. The Committee recommends that careful consideration be given to producing a "Models and Correlations" document for MELCOR equivalent to the similar documents prepared for the NRC's thermal-hydraulic systems codes. At a minimum, the Committee recommends that all new model development be accompanied by detailed documentation of model pedigree, applicability, and fidelity (benchmarking).

A particular area of concern to the Committee is the dispersed nature of model documentation when other computer codes, or parts of other computer codes, have been imported into MELCOR. In several areas, MELCOR documentation simply references the manuals for the parent code without sufficient supporting discussion of which portion(s) of the parent code models have been incorporated unchanged into MELCOR, which have been modified, and how the imported models have been incorporated into the MELCOR framework. The ideal solution would be to incorporate into the MELCOR documentation a complete description of each imported physical model. At a minimum, the Committee recommends that references to parent code documentation in the MELCOR documentation be accompanied by a clear and specific discussions of how and why the imported models have been selected for MELCOR implementation.

The MELCOR developmental assessment documeniation is sparse. The Committee recommends that the MELCOR developmental assessment be expanded and that the results of the assessment effort be thoroughly documented. The Committee recommends that as future assessments are completed, careful consideration be given to documentation of the assessments, individually and collectively, so that the lessons learned in the assessment process are incorporated into the MELCOR development effort and and immediately helpful to the user community.

The design of the MELCOR code, with its strong emphasis on user input for modeling both the facility and parametric studies of the many and complex physical processes being examined, places a uniquely difficult burden on the MELCOR user. Selection of appropriate values for the various parameters used in the phenomenological models is crucial because the representation of physical processes can be markedly influenced by parameter selections. Effective use of MELCOR demands a knowledgeable and well-informed user. Documentation of practicai modeling guidelines is needed. The ongoing collection of user lessons learned or practical guidelines does not seem to be occurring. The Committee recommends that a structured and ongoing process of collecting, documenting, and distributing practical user guidelines to the MELCOR user community be developed and executed. 


\section{INTRODUCTION}

MELCOR (Ref. 1-1) is a fully integrated, engineering-level computer code that models the progression of severe accidents in light water reactor nuclear power plants. MELCOR is being developed at Sandia National Laboratories (SNL) for the United States Nuclear Regulatory Commission (USNRC) as a second-generation plant risk assessment tool and as the successor to the Source Term Code Package. In the two-tier analysis code structure used by the NRC, MELCOR is an upper tier, fast-running integral code in contrast to the mechanistic severe accident codes of the lower tier. A key goal of the MELCOR code is to treat the entire spectrum of se:ere accident phenomena, including reactor coolant system and containment thermal-hydraulic response, core heatup, degradation and relocation, and fission product release and transport, in a unified framework for both boiling water reactors (BWRs) and pressurized water reactors (PWRs). The targeted applications envisioned by the NRC for MELCOR are BWR and PWR probabilistic risk assessment (PRA) studies, audit reviews of Individual Plant Examination (IPE) submittals, studies to develop insights into phenomena and hardware performance, and accident management studies (Ref. 1-2).

MELCOR has been under development since 1982 (Ref, 1-3). ${ }^{*}$ The newest version is 1.8.1, July 1991. The code has now reached sufficient maturity that a number of organizations inside and outside the NRC are using or are planning to use the code. Although the quality control and validation efforts are in progress, there is a need to have a broad technical review by

\footnotetext{
* Reference 1-3, the "MELCOR Primer, Version 1.8.()," is composed of the following individual documents:

- MELCOR Architecture Programmer's Guide

- Machine Dependent Code in MELCOR

- MELCOR/MELGEN

- Users' Guides

- Burn Package

- Cavity Packagc

- Control Function Package

- Corc (COR) Packagc

- Control Volume Hydrodynamics Package

- Decay Heat Package

- Fan Cooler (ESF/FCL) Packagc

- External Data File Package

- FDI Package

- Flow Path

- HS Package

- Matcrial Propertics

- MELPLT Users' Guide

- Non-Condensible Gas EOS

- Radionuclidc Packagc

- Containment Sprays Package

- Tabular Function

- Transfer Process

- Reference Manuals

- Burn Package

- Core (COR) Package

- Thermal Hydraulic Packages (CVG and FL)

- Control Volume Thermodynamics Package (CVT)

- Heat Structure (HS) Package

- Radionuclide Package

- Fan Cooler (ESF/FCL) Package
} 
recognized experts to determine or confirm the technical adequacy of the code for the complex analyses it is expected to perform. A peer review committee has been organized using recognized experts from the national laboratories, universities, MELCOR user community, and independent contractors to perform this assessment.

The objective of this report is to docuraent the findings of the MELCOR Peer Review Committee formed to fulfill the charter described in the following section.

\subsection{Committee Charter}

The charter of the MELCOR Peer Review Committee was to (1) provide an independent assessment of the MELCOR code through a peer review process, (2) determine the technical adequacy of MELCOR for the complex analyses it is expected to perform, and (3) issue a final report describing the technical findings of the Committee.

\subsection{Committee Membership}

The Committee membership was selected with the objective of conducting a broad peer review of the MELCOR code using recognized technical experts from universities, the national laboratories, MELCOR code users, and other technical organizations. There was early recognition by the NRC and the MELCOR Peer Review contractor (Los Alamos National Laboratory) that a variety of backgrounds, skills, and professional experiences must be built into the Committee through its membership. For example, it was recognized that review of the detailed MELCOR models and correlations forming the individual phenomenological packages (e.g., hydrodynamic behavior, condensation, evaporation, conduction, convection, aerosol dynamics, chemistry) was required. The Committee has since called this activity the "bottom-up" or detailed code review. It was also recognized that technical adequacy of the integration of the individual models and correlations into packages and finally into the total code must be assessed. This integration is done within a framework, for example, of the selected conservation equations to be solved, code numerics, and architecture. The Committee has since called this activity the "top-down" or integral code review. Listed below are the Committee members selected to meet these review needs. Brief experience summaries of each Committee member are presented in Appendix A. The committee members are as follows:

Dr. Brent E. Boyack, Los Alamos National Laboratory, Committee Chairman

Dr. Vijay K. Dhir, University of California at Los Angeles

Dr. James A. Gieseke, Battelle Memorial Institute, Columbus, Ohio

Dr. Tim 3 . Haste, United Kingdom Atomic Energy Authority, Winfrith

Dr. Marc A. Kenton, Gabor, Kenton and Associates, Inc.

Dr. Mohsen Khatib-Rahbar, Energy Research, Incorporated

Mr. Mark T. Leunard, Science Applications International Corporation

Dr. Raymond Viskanta, Purdue University

\subsection{Peer Review Process}

The Committee developed and followed a multistep process for the MELCOR Peer Review. A description of the individual steps in the peer review process is provided following a listing of each step in the review process. Although the steps are listed in a logical progression, the Committee notes that some of the steps were visited more than once and some activities were conducted in parallel over a period of time.

1. Identify design objectives for the MELCOR code. The design objectives for the MELCOR code were identified by the NRC in Ref. 1-2. The design objectives for the MELCOR code are as follows: 
MELCOR should appropriately and consistently model phenomena essential to the description of severe light water reactor accidents.

- $\quad$ MELCOR should provide best-estimate predictions of the progression and consequences of severe accidents.

- MELCOR should permit estimates of the uncertainties associated with its predictions of severe core damage accidents to be made without requiring modifications to the code.

- Models incorporated in MELCOR should be in adecuate detail to address the phenomenology but permit a practical running time for accident sequence analysis.

- MELCOR should be applicable for severe accident studies and related plant applications for both PWRs and BWRs.

- $\quad$ MELCOR should be portable, maintainable, and have a structure that facilitates incorporation of new or alternative phenomenological models.

The Committee recognized that definitions of terms such as "appropriately and consistently," "adequate detail," and "practical running time" would need to be developed as a support to individual Committee member efforts to reach joint findings. The Committee did develop supporting definitions to foster common interpretation of the design objectives and these are discussed in Chapter 2.

2. Identify targeted applications for the MELCOR code. The targeted applications for the MELCOR code were identified by the NRC in Ref. 1-2. The targeted applications for the MELCOR code are as follows:

- $\quad$ PRA studies of PWRs and BWRs,

- best-estimate accident sequence studies to develop insights into (1) phenomena and (2) hardware performance,

- NRC audit reviews of IPE submittals,

- $\quad$ accident management studies that analyze the progression of severe accidents and evaluate the effects of given mitigation strategies, both beneficial and detrimental, and

- benchmark simplified source term estimation algorithms such as the NUREG$115)^{\prime} \%$ SOR codes.

The Committee recognized that more detailed descriptions of how MELCOR would be used in each targeted application needed to be developed as a support to individual Committee member efforts to reach joint findings. The Committee did develop more detailed descriptions to foster common interpretation of the design objectives and these are discussed in Chapter 3.

3. Identify the MELCOR code version to be reviewed. The Committee originally planned to focus on a specific code version and did not intend to take into consideration subsequent code revisions subsequent. As it became clear to the Committee that needed and significant code revisions were under way and that development work was likely for several years 
to come, the Committee decided to base its review on a code version issued near the end of the review process, MELCOR Version 1.8.1, July 1991.

4. Identify and distribute the MELCOR Document Data Base to Committee members. An initial MELCOR Document Data Base was compiled at the start of the MELCOR Peer Review process. The MELCOR Document Data Base was updated as new items of information were identified. A listing of the final version of the MELCOR Document Data Base is provided in Appendix B. Included in the MELCOR Document Data Base are (1) Reference Reports, (2) Published SNL Papers, (3) Reports and Papers External to SNL, (4) Correspondence and Memoranda, (5) Other Information, and (6) Committee Documents and Findings.

5. Select plants and severe accident scenarios. The BWR plant selected was LaSalle Unit 2. The PWR plant selected was Surry. The Committee found that there was a limited choice of existing and available MELCOR input decks. The LaSalle Unit 2 MELCOR input deck was developed at SNL; it was used to support the LaSalle PRA effort (Ref. 1-4) and was also used to calculate the BWR severe accident sequence reviewed by the MELCOR Peer Review Committee. The starting point for the Surry MELCOR input deck examined by the MELCOR Peer Review Committee was an input deck developed by the Idaho National Engineering Laboratory (INEL) for a total loss of feedwater transient. SNL acquired and modified the INEL Surry input deck and used the modified input deck to calculate the PWR severe accident sequence reviewed by the MELCOR Peer Review Committee.

As with the selection of BWR and PWR plants, the selection of severe accident scenarios was largely a matter of availability. The LaSalle fully-integrated, full-sequence severe accident scenario selected was a high-pressure, short-term station blackout. This scenario was characterized by (1) loss of all onsite ac and de power, (2) reactor trip and isolation, and (3) failure of all emergency core cooling systems, the automatic depressurization system, and containment heat removal system. This sequence was examined by SNL as part of its involvement in the NRCsponsored Phenomenology and Risk Uncertainty Evaluation Program. The Surry fully integrated, full-sequence severe accident scenario selected was a total loss of feedwater accompanied by a failure of all emergency cor z cooling systems (TMLB' sequence). The original Surry MELCOR input deck created by the INEL was set up for the TMLB' sequence. The SNL Surry calculation was the first full-plant, full-sequence PWR calculation performed with MELCOR by SNL.

6. Develop a common perspective. The Committee members come from a variety of backgrounds and hold a variety of perspectives with respect to deciding "how good is good enough?" "These diverse perspectives are the strength of the peer review committee format. However, it is important that the Committee strive to develop a reasoned perspective regarding expectations of reasonableness. To that end, the Committee identified three important factors related to severe accident phenomena. The development and statement of these factors were an important part of evolving the common perspective needed to review the "technical adequacy" of the MELCOR code. The three factors are as follows.

Knowledge of Physical Processes. The current level of scientific knowledge regarding severe accident processes varies. The physics of some physical processes are well understood while the physics of other physical processes are partially or poorly understood. Stated another way, severe accidents are complex, follow uncertain paths, and include some phenomena that are not well characterized at present. Given the reality that the physics of some severe accident phenomena remain uncertain at present, the Committee cannot expect MELCOR to accurately model that which the scientific community has not yet adequately characterized. The MELCOR code should, therefore, permit the consideration of uncertain physical processes by other means (e.g., sensitivity studies).

Physics. The objective of any modeling effort should be to get the physics correct since incorrect physics cannot predict reality. This objective should apply to any process deemed worthy of modeling in the MELCOR code and for which the physics are known. Models that have incorrect physics but which produce a result close to reality are neither technically adequate or acceptable. The physics of a given phenomena can be modeled at several different levels 
depending on how much of the total characts: of the phenomena is to be captured. The Committee determined that for an integrated severe accident code such as MELCOR, sufficient physics should be present in the model to capture the major features and trends of the dominant phenomena. A model that captures the major features or trends of dominant phenomena and leads to the physically correct end stute was defined by the Committee to be a zeroeth order model. Higher order models improve the characterization of features or trends that affect event timing and magnitude and more accurately represent the passage to the end state.

Importance. Processes occurring during a severe accident are not of equal importance. Some processes are very important in determining the major features and trends of a severe accident while others are of moderate or minimal importance. The issue of importance is taken into consideration when either (1) severe accident physics are incorrectly modeled or (2) the sensitivity of results to uncertain severe accident physics cannot be considered. If either (1) or (2) are the case but it can be determined that the phenomenon is of little importance in influencing the major features, trends, or outcome of a severe accident transient, then the technical adequacy of the code is not adversely affected. However, importance statements such as these have more relevance to affixing priorities as to when these defers should be corrected than to condoning their continuance.

7. Identify dominant phenomena for the plants and scenarios. During the course of the Committee's efforts, a significant amount of time was devoted to identifying dominant phenomena for the plants and scenarios chosen in steps 5 and 6 . The objective of this exercise was (1) to identify and list severe accident phenomena, (2) identify important or dominant severe accident phenomena, and (3) check for the existence of MELCOR models for the dominant severe accident phenomena. Although the goals of this step are important, the Committee did not have the resources to fully implement this step. Rather, having identified and listed the severe accident phenomena, no formal ranking activities were conducted to identify the dominant phenomena. Thus, the phenomena appearing in the full list were used by the Committee members in a less formal manner to determine if a complete set of models was present in each phenomenological package. The list of phenomena generated by the Committee is presented in Appendix C.

\section{Define a "Standard of Technical Adequacy" to be used in developing} findings. The Committee felt that it was important to define a standard against which technical adequacy could be measured. Such a standard, and the discussions that accompanied development of the standard, helped the individual Committee members evolve toward a common definition of technical adequacy. Because a key element of the Committee charter was to "determine the technical adequacy of MELCOR for the complex analyses it is expected to perform," developing a common Committee view of what constituted "technical adequacy" was important. The Committee developed definitions of technical adequacy for the detailed models in MELCOR (the bottom-up view) and for the total or integrated MELCOR code (the top-down view).

The Committee standard for technical adequacy of the individual MELCOR models is that (1) the model pedigree is known, documented, and acceptable, (2) the model is used appropriately or stated in another manner, the application of the model is acceptable, and (3) the prediction of, or fidelity to, the dominan phenomena modeled is acceptable. A brief explanation of each of these elements of technical adequacy of the individual MELCOR models follows.

Model pedigree is based upon the model origin; the basis for the model including physical reasonableness, assumptions, restrictions, simplifications; the original application of the model and its relationship to the MELCOR application; and the experimental or other data base supporting the model.

Model applicability builds on the concept of pedigree, but, in addition, is based upon the relationship of the model to the dominant phenomena being modeled and the existence of acceptable justification if the model is used beyond the original pedigree.

Model fidelity builds on the concepts of pedigree and applicability, but, in addition, is hased upon the ability to predict dominant phenomena with acceptable accuracy. Thus, the key element of fidelity is benchmarking of the model against other standards. The highest level 
standards are exact solutions of well-defined physical problems, followed in descending order of desirability by prototypic data, other data, code-to-code comparisons, and expert opinion.

The Committee standard for technical adequacy of the total or integral code is that (1) the total code is applicable, and (2) the prediction of integral phenomena is acceptable. A brief explanation of each of these elements of technical adequacy of the total MELCOR code follows.

MELCOR applicability is based upon finding that several important features of the integrated code are acceptable. These features are adequacy of the global field or conservation equations, adequacy of the individual models for the dominant phenomena as determined from the bottom-up review, adequacy of the global numeric techniques, and adequacy of the plant/reactor/components/auxiliaries modeling and noding capability.

MELCOR fidelity builds on the concept of MELCOR applicability, but, in addition, is based upon the ability of the code to predict integral plant behavior with acceptable accuracy. Thus, the key element of fidelity is benchmarking of the total code against other standards. Again, the highest level standards are exact solutions of well-defined physical problems, followed in descending order of desirability by prototypic data, other data, code-to-code comparison, and expert opinion. Exact solutions and prototypic integral data are sparse at this time. Thus, it is necessary to rely more heavily on comparisons to experiments having subsets of the full integral behavior being examined, code-to-code comparisons, and expert opinion.

9. Define a process for reviewing for technical adequacy. Having defined the Standard of Technical Adequacy, the process for reviewing for technical adequacy was defined.

A bottom-up review is conducted by examining the pedigree, applicability, and fidelity of the many individual models and closure relationships in MELCOR. This is most easily accomplished if a "Models and Correlations" document exists. Examples of such documents for thermal-hydraulics systems analysis codes are found in Refs. 1-5 and 1-6. For the bottom-up review, the Committee developed a set of classification definitions based upon the elements of perspective discussed in step 6. Findings of Categories 1,2, and 3, as defined in Table 1-I, are findings of technical adequacy. Findings of Categories 4, 5, and 6, as defined in Table 1-1, are findings of technical inadequacy. A finding of Category 7 , as defined in Table 1-I, is a finding of technical inadequacy but low importance.

A top-down review is conducted by examining the applicability and fidelity of the total code. This is most easily accomplished if an extensive benchmarking effort has been conducted and documented for the version of the code being reviewed. In the absence of such information, code-to-ce le comparisons and expert opinions become stronger elements of the lop-down review.

10. Assess technical adequacy of individual models and/or correlations within the MELCOR phenomenological packages (bottom-up review). The Committee conducted the required reviews of the individual models and correlations. Review findings, summarized for each MELCOR phenomenological package, are presented in Chapter 2. More detailed descriptions of the models and correlations within each MELCOR phenomenological package are found in Appendices $D$ through $K$. Lead review responsibilities were assigned for each phenomenological package. Additional Committee members participated in the review of several phenomenological packages. Review responsibilities for the phenomenological packages are tabulated in Table 1-II. 
Table 1-I

MELCOR Bottom-Up Review

Classification of Findings

\begin{tabular}{|c|l|}
\hline Finding & \multicolumn{1}{|c|}{ Definition } \\
\hline Category 1 & $\begin{array}{l}\text { The severe accident phenomena are generally understood and the physics } \\
\text { are correctly represented with at least a zeroth order model as defined in } \\
\text { step } 6 \text { above. }\end{array}$ \\
\hline Category 2 & $\begin{array}{l}\text { Questions exist regarding the severe accident phenomena but reasonable } \\
\text { physics (in the context of expert judgment) are represented with at least a } \\
\text { zeroeth order model. }\end{array}$ \\
\hline Category 3 & $\begin{array}{l}\text { Questions exist regarding the severe accident phenomena and no model is } \\
\text { provided. However, features are available in the code to perform } \\
\text { sensitivity studies to represent the currently understood ranges of } \\
\text { phenomenological behavior. }\end{array}$ \\
\hline Category 4 & $\begin{array}{l}\text { The severe accident phenomena are generally understood but the physics } \\
\text { are not correctly represented by models in the code. }\end{array}$ \\
\hline Category 5 & $\begin{array}{l}\text { Questions exist regarding the severe accident phenomena but reasonable } \\
\text { physics (in the context of expert's judgment) are not represented by } \\
\text { models in the code. }\end{array}$ \\
\hline Category 6 & $\begin{array}{l}\text { Questions exist regarding the severe accident phenomena and no model is } \\
\text { provided. Features are not available in the code to perform sensitivity } \\
\text { studies to reprisent the currently understood ranges of phenomenological } \\
\text { behavior. }\end{array}$ \\
\hline Category 7 & $\begin{array}{l}\text { A finding of either Category 4, 5, or 6 would apply but the phenomenat } \\
\text { being modeled are not of sufficient importance to markedly influence } \\
\text { either the major features or trends of the severe accident. However, } \\
\text { importance statements have more relevance to affixing priorities as to } \\
\text { when defects should be corrected than to condoning their continuance. }\end{array}$ \\
\hline
\end{tabular}

11. Assess technical adequacy of the integral code against the MELCOR design objectives and the MELCOR targeted applications (top-down review). The Committee conducted the required reviews of the total, integrated code. These findings are presented in Chapters 2 and 3 within the context of the MELCOR design objectives and targeted applications. The MELCOR design objectives were previously identified in step 1 above. The MELCOR targeted applications were previously identified in step 2 above.

12. Document findings in a summary report. This report documents the findings of the MELCOR Peer Review Committee and the process used to develop those findings.

\subsection{Presentation of Committee Findings}

The Committee deliberated the manner in which its findings were to be communicated. The Committee decided to present its primary findings relative to the code design objectives and the targeted applications as described by the NRC in Ref. 1-2. The findings relative to the code objectives are presented in Chapters 2 and 3; findings resulting from both the bottom-up and topdown reviews are covered. The findings relative to the targeted applications identified by Ref. $1-2$ are presented in Chapter 4 . Other review findings that could not be naturally placed in Chapters 2 , 3 , or 4 are provided in Chapter 5. Committee recommendations are presented in Chapter 6 . The appendices provide more detailed descriptions of the summary information presented in the body of the report. 
Table 1-II

Review Responsibilities for MELC(OR

\begin{tabular}{|l|l|l|}
\hline MELCOR Package & Lead Reviewer & Other Reviewers \\
\hline Hydrodynamic Behavior & M. Khatib-Rahbar & $\begin{array}{l}\text { D. R. Liles (LANL) } \\
\text { V. K. Dhir }\end{array}$ \\
\hline Heat Structure & V. K. Dhir & R. Viskanta \\
\hline Core Heatup and Degradation & R. Viskanta & $\begin{array}{l}\text { T. J. Haste } \\
\text { M. Khatib-Rahbar }\end{array}$ \\
\hline $\begin{array}{l}\text { Fission Product and } \\
\text { Aerosol Transport }\end{array}$ & J. A. Gieseke & $\begin{array}{l}\text { M. T. Leonard } \\
\text { M. A. Kenton } \\
\text { C. Alexander (BCL), chemistry }\end{array}$ \\
\hline Fuel Dispersal Interactions & V. K. Dhir & V. K. Dhir \\
\hline Core-Concrete Interactions & M. Khatib-Rahbar & Riskanta \\
\hline Gas Combustion & M. A. Kenton & M. T. Leonard \\
\hline Engineered Safety Features & M. A. Kenton & \\
\hline Properties & T. J. Haste & D. R. Liles (LANL) \\
\hline Transfer Processes & T. J. Haste & \\
\hline & $\begin{array}{l}\text { M. A. Kenton } \\
\text { M. T. Leonard } \\
\text { B. E. Boyack }\end{array}$ & Remaining Committee members \\
\hline Integral Code &
\end{tabular}

\section{REFERENCES}

1-1. R.M. Summers, R.K. Cole, Jr., E.A. Boucheron, M.K. Carmel, S.E. Dingman, J.E. Kelly, "MELCOR 1.8.(): A Computer Code for Severe Nuclear Reactor Accident Source Term and Risk Assessment Analyses," Sandia National Laboratories report SAND-9()0364 (NUREG/CR-5531) (January 1991).

1-2. F. Eltawila, Nuclear Regulatory Commission, letter regarding NRC statement of MELCOR objectives and targeted applications to B. E. Boyack, Los Alamos National Laboratory (November 2, 199() ).

1-3. R. M. Summers, "MELCOR Primer, Version 1.8.0," Sandia National Laboratories unnumbered report (February 17, 1989).

1-4. "Integrated Risk Assessment for the LaSalle Unit 2 Nuclear Power Plant: Phenomenology and Risk Uncertainty Evaluation Program (PUREP), Volume 3: MELCOR Calculations," Sandia National Laboratories report SAND90-2765RX, (NUREG/CR-5305) (December 1990)).

1-5. D. R. Liles, J. W. Spore, T. D. Knight, R. A. Nelson, M. W. Cappiello, K. O. Pasamehmetoglu et al., "TRAC-PF1/MOD1 Correlations and Models," Los Alamos National Laboratory report LA-11208-MS; (NUREG/CR-5069) (December 1988).

1-6. "RELAP5/MOD3 Code Manual, Vol IV: Models and Correlations," EG\&G Inc. report EGG-2596 (NUREG/CR-55.5) (June 199()). 


\section{BO'TTOM-UP REVIEW OF MELCOR PHENOMENOLOGICAL PACKAGES}

Detailed reviews of each MELCOR phenomenological package are presented in Appendices D through $K$. However, the Committee decided that a summary of the phenomenological package reviews was advisable and the objective of this chapter is to present such reviews of each phenomenological package. Each summary review is divided into three elements: (1) a brief description of the phenomenological package, (2) a qualitative perspective that considers, for example, factors such as the level of modeling detail, whether the models were developed for MELCOR or imported, and which parts of the package are judged to be either adequate or inadequate, and (3) a discussion of technical adequacy issues including a list of highpriority Category 4 and 5 models (defined in Table 1-1) and high-priority missing models needed for the PRA-targeted application. The order in which the phenomenological package summaries are presented is as follows:

1. Control Volume Hydrodynamics (CVH)/Flow Path (FL)

2. Heat Structure (HS)

3. Core (COR)

4. Radionuclide (RN)

5. Fuel Dispersal Interaction (FDI)

6. Core-Concrete Interactions (CAV)

7. Gas Combustion/Burn (BUR)

8. Engineered Safety Features (ESF)

9. Properties

\subsection{CVH/FL Phenomenological Package Review Summar,}

\subsubsection{Phenomenological Package Description}

In MELCOR, the thermal-hydraulic processes are modeled by the Control Volume Hydrodynamics (CVH) and Flow Path (FL) packages, while the thermodynamic calculations are performed within the Control Volume Thermodynamics (CVT) package. The CVH/FL packages are based on a highly versatile architecture and a general control volume hydrodynamic network concept that provide thermal-hydraulic boundary conditions to other MELCOR phenomenological packages.

A general "volume/altitude" and "virtual volume" approach is employed to define, through user input, the control volume geometry. Hence, component and subsystem models must be built in through user input. This concept provides a valuable tool for application of MELCOR to a variety of nuclear reactor designs.

Control volumes are interconnected via "flow paths" through which hydrodynamic material may pass without any residence time (assumption of negligible volume). Flow path area can be modified by input to model valves, obstructions, etc. The material and energy contents of both coolant and noncondensable gases are assumed to reside within control volumes.

Mass and energy sources and sinks are treated as boundary conditions to CVH/FL. This includes decay heat, heat from structures, water from condensation and evaporation on structures, and noncondensable gas sources from core-concrete-interactions (CAV), oxidation, and other sources.

In CVH/FL, hydrodynamic materials are assumed to separate by gravity into a lower pool region (which may contain steam bubbles but not noncondensables), and an overlying atmosphere (which may contain liguid droplets, gases, and vapor). The pool and atmosphere velocities and directions may be different.

The mass exchange models include options for (1) a thermal and mechanical equilibrium model, which assumes the same pressure and temperature for both pool and atmosphere, and (2) a thermal noneguilibrium model, which assumes the same pressure but different temperatures for pool and atmosphere (vapor superhealt and licyuid subcooling). 
The basic hydrodynamics methods embodied within the CVH/FL package are closer in principle to those of RELAP4 than TRAC or RELAP5 computer codes. The CVH/FL models are an adaptation of the approach employed by HECTR and CONTAIN containment analysis codes.

\subsubsection{Qualitative Perspective}

The inherent limitations of the well-mixed control volume approach in simulation of truly multidimensional phenomena need to be made very clear as part of the CVH/FL documentation and manuals. Other issues potentially impacting CVH/FL predictions include:

- modeling of transport in pipe-like geometries is susceptible to numerical diffusion, which is typical of most computer codes which use similar methods;

- difficulties in defining control volume velocities for use in closure relations, when several flow paths are connected to any control volume;

- difficulties in defining characteristic dimensions, loss coefficients, etc, when dividing a large, unobstructed volume into several interconnected control volumes and flow paths; and

- difficulties in predicting stratification (with and without noncondensables) using the $\mathrm{CVH} / \mathrm{FL}$, requiring sufficiently detailed nodalization together with some knowledge of expected flow patterns.

Calculations performed to date for both BWRs and PWRs have shown that the CVH/FL modeling approach appears to be adequate under most severe accident conditions; hovever, considerable insights on the part of code users are required. The reliability of MELCOR-calculated results is highly questionable in light of the existing numerical problems, because it is often difficult to distinguish between numerical vs physical behavior (the origin of these numerical problems is not clearly known, even though some potential problems may exist in the CVH/FL package numerics, especially in terms of CVH/FL coupling to the other MELCOR phenomenological modules).

\subsubsection{Technical Adequacy}

A prioritized summary list of Categories 4 and 5 as well as other important review findings follows:

Numerical Problem and Code Reliability Numerical approximations, averaging schemes, and absence of spatial derivative flux terms of the momentum equations, together with "loose" coupling between the $\mathrm{CVH} / \mathrm{FL}$ and other MELCOR phenomenological packages can exacerbate the MELCOR numerical problems reported by several users.

Natural Circulation. The present model does not include provisions for simulation of buoyancy-induced recirculation within the core and the upper plenum, countercurrent flows in pipes, and circulation/mixing within steam generator tubes and plena.

Impact of Blockage Formation on Flow Diversion. Impact of blockage formation within the core region following core degradation on hydrodynamic calculations can only be partially treated |flow area changes through the control functions/tabular functions (CFs/TFs)|. This can strongly impact in-vessel flow circulation, hydrogen generation, and core melt progression.

Condensation at Pool/Atmosphere Interface. The present pool/atmosphere steam condensation model is incorrect and needs to be corrected. Overprediction in condensation rates is expected for conditions such as a steam atmosphere overlying a large quiescent water pol.

Pressurizer Drainage Model. The present approach to momentum transfer between pool and atmosphere phases within flow paths needs further assessment. This model is inadeyuate for prediction of pressurizer drainage under PWR severe accident conditions. 
Additional ESF Model. Specific models for pressurizer sprays, BWR core sprays, and flows through ice beds are not currently available within CVH/FL or other MELCOR phenomenological packages.

Reflond. The present hydrodynamics model cannot model reflood properly. This limits applications of MELCOR to assessment of success criteria and accident managements (AM).

Compendium of Sensitivity Studies, Parametric sensitivity studies with CVH/FL in particular, and MELCOR in general, are for the most part not available. Various model options should be tested and the results documented for use by MELCOR code users.

Experimental and Code Benchmarking. There is insufficient experimental benchmarking of the CVH/FL packages. The ultimate judgment for determination of the adequacy of hydrodynamic models requires more extensive comparisons with both separate-effects and integral tests.

Improved Documentation. Specific guidance to users is essential. For example, the junction opening heights determine what flows through a flow path. Specification of junction opening heights is subject to some interpretation, requiring additional guidance in order to avoid potential misinterpretations. A better documentation of bases for correlations and models is also essential.

\subsection{HS Phenomenological Package Review Summary}

\subsubsection{Phenomenological Package Description}

The Heat Structure (HS) package is used in the MELCOR code to determine the thermal response of the structures present in the reactor coolant system (RCS) and in the containment. The package serves as a building block in several other packages in the code. The geometry, physical dimensions, and thermophysical properties of heat structures are user input. The one-dimensional transient conduction equation with volumetric heating is solved using a finite-difference scheme to obtain the temperature distribution in the structures. A provision for specifying temperature, heat flux, or convective boundary condition exists. For convective boundary conditions, steady-state correlations well documented in standard heat-transfer text books have been used. The submodels included in this package are as follow:.

Thermal Properties, Only thermal conductivity and volumetric heat capacity are considered. Temperature-dependent values are obtained from the Material Properties Package. Radiative properties are specified through user input.

Atmosoheric Convection Heat Transfer. Both internal and external convection under free and forced convection in rectangular, cylindrical, and spherical geometries are considered. Correlations for fully developed steady-state flows are used.

Atmospheric Radiation Heat Transfer Equivalent band or gray gas model options are employed. View (shape) factors are arbitrarily specified.

Power Sources. Both external and internal heat sources in a heat structure are included. The internal heat sources can be spatial and time dependent. Surface heat fluxes are user specified.

Pool Fractions. The fraction of surface that is exposed to liquid pool and environment is obtained through geometrical relationships. The concept of critical pool fractions is used to distinguish between environment and the pool.

Pool Boiling Heat Transfer. Correlations for saturated nucleate boiling, maximum heat flux, minimum heat flux, and film boiling are used. Transition boiling heat fluxes are interpolated between critical and minimum heat fluxes.

Mass Transfer Condensation of pure vapor, condensation, and evaporation in the presence of noncondensables and flashing of superheated liquid layers present on the heat structure are considered. For condensation in the presence of noncondensables, a low mass-transfer limit is invoked. 
Liquid Film. Steady-state and transient thickness of liquid film on a structure are obtained through Nusselt-type film condensation coefficients. Arbitrurily specified default values are used for initial film thickness and for the maximum film thickness.

Stored Energy. Energy stored in the structure and in the liquid film on the suiface is calculated. The energy content is obtained by integrating over the volume, the product of temperature, density, and specific heat.

Degassing Model. Gas release rate during decomposition of a solid is calculated. Over the temperature range of decomposition, the gas release rate is assumed to vary linearly.

\subsubsection{Qualitative Perspective}

The correlations used are well tested and are based on large amounts of laboratory data obtained under a variety of conditions. However, the correlations may not always be applicable to the physical situation being modeled. Since accident progression is a transient process, use of steady-state correlations can always be questioned especially when the transients are severe. A similar comment can be made with respect to geometrical differences that may exist between the bases of the correlations and the applications. Some of the correlations are dated, but could easily be updated without significant effort. Correlations for some of the situations such as flow boiling and mixed convection are not available in the package, but could be added with little effort. Once a boundary condition is specified, the fidelity of the results depends on the numerics. Transient calculation in one of the sample problems (semi-infinite slab) showed oscillation in temperature. The magnitude of the temperature oscillations was dependent on the spatial node and time-step size.

\subsubsection{Technical Adequacy}

The modeling of condensation in the presence of noncondensables is physically not correct as film thermal resistance is neglected. Also, for all situations the low mass-transfer limit is invoked. This model should be corrected, otherwise the results from the code with respect to containment response will always be suspect.

At high temperatures radiation heat exchange between structures can be very important witl, respect to the determination of the heatup rate of RCS. In the package no models exist for calculation of shape factors. The models or correlation for shape factors should be added.

Several of the correlations used in the package are dated. Possibility of improper use of steady-state fully developed flow correlations to transient and developing flows exists. Also, no guidance is given to the user as to what constitutes an appropriate characteristic length.

Numerical oscillations observed in the test problem may affect calculations in other packages since the HS package is a building block. The oscillations may worsen if the convective boundary condition (e.g., flashing) represents a rapid transient situation.

\subsection{COR Phenomenological Package Review Summary}

\subsubsection{Phenomenological Package Description}

COR is one of the most important phenomenological packages of the MELCOR code. The package describes the thermal response of structures in the core/lower plenum regions and the heat transfer from these structures to the surrounding thermal-hydraulic volumes, the melting and relocation of the core materials within the core and the lower plenum, and the lower head penetration failure and debris ejection. The COR package treats all important modes of heat transfer in the core and calculates decaly heat generation rates. In addition, the package also considers metal oxidation, core debris formation, and relocation. Lower head heatup, breach, and debris ejection are also modeled. The treatment of the phenomena is rather uneven. As expected, well-understood phenomena are modeled realistically, whereas poorly understood phenomena are handled parametrically. (See Table F-I in Appendix F for summary of phenomenological models.) 
The COR package models dominant phenomena involved in core degradation during a severe nuclear reactor accident. The phenomenological and parametric models describe core heatup, metal oxidation and hydrogen generation, cladding failure and fission product gap releases, core melting, slumping and blockage, debris bed formation, lower head and penetration failure, and molten debris ejection from the breached vessel. Some of the more important phenomena treated by the package are highlighted in the following.

Intact Core Heatup Heat sources include fission power, decay power, and oxidation heat generation. Heat transfer paths include conduction inside core structures, convection between structures and coolant, and radiation exchange among the structures and the surrounding steam. Local fluid temperature is calculated using a one-dimensional model for assumed unidirectional flow) to provide axial fluid temperature distribution within a coarse hydrodynamic cell.

Metal Oxidation. Oxidation of Zircalloy and steel in a high-temperature steam environment is modeled using parabolic kinetics with appropriate reaction rate constants. A boron carbide oxidation model is included but is currently disabled. The control function may be used to shut off oxidation to simulate the effects of flow blockage.

Hydrogen Generation. Hydrogen generated from the oxidation reaction in hightemperature steam environment is modeled. Steam starvation may not be predicted correctly because of the use of coarse hydrodynamic cells, multidimensional flow resulting from flow blockage, etc.

Cladding Failure Cladding failure and fission product gap releases are described by the temperature criterion through user input in the RN package. There are no cladding deformation, ballooning, and shattering models in the COR package.

Core Melting Progression. Melting of a core material occurs at an individual melting temperature of the material. Candling of molten core material, formation of flow blockage, relocation of intact core material with the molten material, and holdup of molten material by the protective oxide shell are handled parametrically. Downward relocation of particulate debris by gravitational settling is modeled not mechanistically but by a logical sequence of processes through consideration of volume, porosity, and support constraints. Oxide shell is assumed to fail if the oxide thickness is reduced below a user-specified value and if a user-specified critical temperature is exceeded.

Lower Head Failure, Heat transfer from a uniform temperature debris bed to the lower head is modeled parametrically through user-specified heat-transfer coefficients. The lower head is heated in an essentially one-dimensional manner, and lateral debris movement and head curvature are not modeled. Thermal (not thermo-mechanical) failure of lower head and penetrations is modeled. The user may specify control functions to simulate the lower head failure caused by direct over-pressure shock from lower plenum steam explosion if the steam explosion can be modeled in some other calculation package.

De ris Ejection. The head is assumed to fail and produce an opening with the initial diameter specified by the user whenever the temperature of the penetration or innermost lower head node reaches a failure temperature specified by the user. Mechanical loading of the head is not considered in the failure mode, although this phenomenon could be modeled using control functions. The velocity of the material ejected is calculated from the Bernoulli equation with a user-specified discharge coefficient. An attempt is made to update the diameter of the penetration failure with time.

\subsubsection{Qualitative Perspective}

Many of the identified core degradation and in-vessel melt progression phenomena are modeled in the COR package but treatment of some important phenomena is missing. The treatment of modeled physical processes is uneven. Well-understood phenomena are treated realistically, but some known processes are modeled only parametrically or not at all.

Some of the models are well established with very little uncertainty. Heat conduction, convection heat transfer for intact geometry, and fission power generation belong to this category (Category 1 ). 
For some phenomena, detailed phenomenological models and experimental data exist; however, because of the code design philosophy and mission (e.g., a fast-running PRA and AM tool), much simpler models are used in the package. The axial heat conduction model across the gas/liquid interface, pool boiling curve correlation for convective heat transfer, metal oxidation, and gravitational settling models should be assigned to this category (Category 2 ).

The models that describe severe core disruption (melting, melt progression, relocation, and blockage) are very crude and parametric in nature. They are not more than educated guesses and represent code developers' views of the phenomena. These models contain little physics because of inherently incoherent processes, describe complicated geometry changes during core degradation and physical/chemical interactions, and lack a definitive experimental data base. These models are mere shells for performing parametric calculations. Models for molten material relocation, blockage formation and holdup by oxide shells, particulate debris formation, solid material transport mechanics of particulate debris, and characteristics of ejected debris should be assigned to this category (Category 3 ).

Natural circulation in the melt pool and crust rupture and melt-down are not modeled in the COR package. Besides the lower head/penetration failure, no other failure location in the RCS pressure boundary is modeled in the package. For the high pressure TMLB' sequence, natural circulation might result in the creep failure of the hot leg, hot-leg nozzle or the surge line before failure of a lower head in a PWR. Thus, the potential of lower pressure melt ejection upon the lower head failure might not lead to direct containment h(ating (DCH). The initial cladding failure, fission product gap release, the potential oxidation of the inner cladding surface, cladding ballooning, and the potential coolant flow diversion because of cladding ballooning are not modeled in the COR package. These issues and those of the melt crust rupture as well as the lower RPV failure mode belong to Category 4.

The transport of solid (unmolten) materials $\left(\mathrm{ZrO}_{2}, \mathrm{UO}_{2}\right.$, steel oxide, or control poison) via the candling process ignores eutectic formation through reaction between pairs of materials such as $\mathrm{UO}_{2} /$ Zircalloy and Zircalloy/Inconel (spacer grids), which lowers the temperature at which melt formation starts and affects the amount of material relocated. For example, the former reaction leads to liquefaction of $\mathrm{UO}_{2} 1000 \mathrm{~K}$ below its melting temperature. The eutectic formation of $\mathrm{U}-\mathrm{Zr}-\mathrm{O}$ will result in the relocation of dissolved fuel with the liquefied molten metal cladding upon breach of the protective $\mathrm{ZrO}_{2}$ shell. The fuel content in the relocated debris will affect the later phase debris heatup, debris oxidation, and fission product release. Although the solid material transport model has a provision to simulate the relocation of fuel accompanied with the molten cladding, the approach does not account for the physical processes. The gap release is only modeled by the use of an input cladding temperature (in the RN package). In BWR control blades, a eutectic alloy can form between $\mathrm{B}_{4} \mathrm{C}$ and stainless steel that liquefies the cladding at temperatures as low as $200 \mathrm{~K}$ below the stainless steel melting point. The subsequent relocation of the liquefied material might form blockages and inhibit boron oxidation. The $\mathrm{B}_{4} \mathrm{C} /$ stainless steel eutectic formation is also not modeled in the COR package. Issues of the eutectic formation, melt crust rupture and the RPV failure mode belong to Category 5.

The core melt progression, slumping, and blockage models are less detailed, whereas the lower head, penetration, and debris ejection models are similar in detail to the models in SCDAP/RELAP5 for treating severe accidents. Although detailed comparison of MELCOR and SCDAP/RELAP5 predictions have not been made for LWR accident sequences, except for TMI-2, some comparisons have been made for small-scale experiments. Such comparisons should be considered with caution since SCDAP/RELAP5 has not been fully benchmarked or validated for transient conditions.

\subsubsection{Technical Adequacy}

Our current state of knowledge with respect to key phenomenological issues related to invessel core melt progression, core debris relocation, lower head attack, and breach are not reflected in the COR package. It should be noted, however, that some of the data has been obtained since the code development was initiated. The main technical findings related to the package can be summarized as follows. 
- Parametric models dealing with core melt progression, core debris relocation into the lower plenum, and lower head attack and failure have no record of peer review. Benchmarking and validation of these models against test data or more detailed phienomenological models do not exist.

- Parametric models are deficient in such areas as time resolution and dynamics. They cannot be validated or improved even after test data become available.

- In many models assumptions and idealizations that have been made are not clearly articulated, and in other cases information needed is not provided in the documents.

- Inadequate modeling of important phenomena in the COR package, or perhaps even worse, areas where models do not currently exist adversely impact performance of PRAs.

\subsection{RN Phenomenological Package Review Summary}

\subsubsection{Phenomenological Package Description}

The Radionuclide (RN) package calculates the release and transport of fission products throughout the core region, the primary system, containments, and the reactor and auxiliary buildings. In addition to tracking fission products, any fuel or structural materials including portions of concrete vaporized or aerosolized during in-vessel melting or during core-concrete interactions are also tracked. The package generates information on airborne concentrations of vapors and aerosols as well as deposited amounts by material class. Material classes are groupings of fission products that have similar properties. The transfer of mass among the vapor, aerosol, and deposited fission product states is modeled as well.

A number of independent but interfaced models comprise the RN package. The package requires input from other MELCOR packages, particularly to obtain information about geometries, flow rates, temperatures (e.g., fuel, pool, surfaces, gases), pressure, physical properties (e.g., gas viscosity), mass transfer coefficients, and amount of water to be condensed in a control volume. The models provide analyses in the major technical areas of (1) fission product mapping, location, and releases, (2) aerosol dynamics, (3) vapor condensation and evaporation, (4) decay heat power, and (5) fission product chemistry.

\subsubsection{Qualitative Perspective}

Fission product mapping, location, and release models deal primarily with the initial set up of the problem, specification of material classes, release from the fuel, and release during core-concrete interactions. The procedures for specifying initial fission product locations and groups are straightforward. Release from the fuel is predicted with the CORSOR/CORSOR-M code which is based on older data and is of limited versatility (Ref. 2-1). Newer data and correlations based on these data are now becoming available. These new correlations shouid handle time-dependent releases more accurately. Further improvements to allow for fuel burn-up and a more representative modification to Te release based on zirconium oxidations should be straightforward. Extension of the available release models to slumped or pooled fuel is questionable because there seems to be a lack of data for release from other than intact fuel pin geometries. Release from core-concrete interactions is treated by a very early version of VANESA known to have errors and limitations. Upgrade to a newer version is recommended. The VANESA code computes the vaporization of fission products into bubbles rising through the melt. The condensation of these vapors is the major source of aerosols released during core concrete interactions although the bursting of the bubbles at the melt surface is an atomization process and a contributor to the total release. A major limitation is the uncertainty in activity coefficients for fission products in the melt. These coefficients can control predictions of release rates. It is likely that sufficient data may never be available on activity coefficients to give validation of releases for all materials during core-concrete interactions; however, ongoing studies continue to provide data 
and guidance. An additional uncertainty arises from the need for oxygen potential in the VANESA release calculations and the fact that $\mathrm{H}_{2} / \mathrm{H}_{2} \mathrm{O}$ ratics may not te accurately predicted.

Aerosol dynamics covers particle ag,glomeration, water condensation or evaporation, deposition, and resuspension. The agglomeration process is well modeled by traditional methods and the MAEROS code has been shown to be adequate in this regard (Refs. 2-2, 2-3, and 2-4). A potential problem is MELCOR's separation of the water condensation or evaporation calculation from the aerosol growth calculation. The CVH package determines the water to be condensed and this value is input to the RN package. A validation of this calculational process has not been pursued but could and should be by checking against more accurate calculations and by comparison with data. In addition, the effect of dissolved ions (vapor pressure reduction) on water condensation should be evaluated for importance and probably added to the calculational procedure.

Particle deposition models used in the various calculations are suitable for large volumes such as a containment. Models for particle deposition from the flows and geometries characteristic of a primary system or reactor vessel internals are not included in MELCOR. These deposition processes include inertial deposition from turbulent flow, diffusional deposition from turbulent flow, impaction from flow direction changes, and impaction on obstacles (e.g., in reactor vessel, steam separator, and steam dryer). Such models are available in the literature and should be added. Other significant omissions are the effect on vapor pressure of dilution in mixed deposits, and the interaction of these deposits with surfaces (chemisorption). Approximate or simple models for these effects could be implemented easily in MELCOR. The flow-induced resuspension model is currently not implemented but this is not serious because resuspension in this manner is expected to be insignificant.

Vapor condensation and evaporation is treated as a straightforward mass-transfer process based on an analogy to heat transfer. The mass-transfer coefficients are imported from the HS package. The analysis is adequate but could be improved by allowing for dilution effects that reduce the vapor pressure of deposits and by correcting the implementation in MELCOR.

Decay heat power modeling is based on an intuitive and user-specified distribution of thermal energy which uses the amounts of decay heat available in the deposit and in the gas, and allows for their apportionment according to surface area and the location of the heat source. The model seems adequate and permits the use of parameters to simulate a variety of assumed situations.

Fission product chemistry is not modeled in a kinetic sense. Allocations of materials into groups with specific chemical and physical properties are handled in an ad hoc fashion. Without a kinetic evaluation of chemistry, the current apprach is adequate. However, there is currently a considerable amount of information available on aqueous chemistry that should form the basis for a first-order model at least for iodine.

\subsubsection{Technical Adequacy}

The $\mathrm{RN}$ package is a mixture of models ranging from highly sophisticated and accurate to very simple representations of physical or chemical processes. In some cases adequate models are missing and unavailable, and improved models are available and could be inserted as improvements. Among the potentially significant models noted as missing but available are those correlations that permit predictions of particle deposition in the reactor coolant system or on reactor vessel internals from directed flows. Important models that are in need of assessment include those that treat water vapor condensation and evaporation since these processes impact strongly on fission product behavior. Also at issue are the condensation/evaporation rates of the fission products themselves where questions exist regarding the reduction of vapor pressures at surfaces by dilution and chemisorption effects as well as the implementation of the basic equations within MELCOR. Modeis for fission product release from the fuel lack time dependence and are outdated. Prediction of release rates of fission products during core-concrete interactions would benefit from model improvements and from a closer coupling to the thermodynamics calculations.

The general approach taken in the RN package seems to be adequate, but improvements having substantial impact while requiring only relatively small efforts could and should be made. 
A potential problem area regards the lack of a process to verify the conservation of mass by the RN package. This has been demonstrated to be a problem and needs attention. The models most in need of attention are those that deal with particle deposition from flows, which are missing, those that interface water condensation/ evaporation to particle growth, which need to be assessed, those that compute fission product condensation/evaporation, which are incorrectly implemented, and those that deal with fission product release both in-vessel and during core-concrete interactions.

\subsection{FDI Phenomenological Package Review Summary}

\subsubsection{Phenomenological Package Description}

Only a limited model of fuel dispersal interactions (FDI) is contained in the MELCOR code. The mixing phase of the interaction of the low-pressure melt ejected from the vessel with the water in the cavity is considered. A Weber number criterion is used to determine the size of the broken up molten material. Heat transfer from the molten material to water is assumed to occur by radiation. All of the energy is utilized in evaporation of water. No heat loss from the molten material is considered if water is not present. This model interacts with the COR and CAV packages.

\subsubsection{Qualitative Perspective}

A Weber number criterion does represent a logical approach to describe ex-vessel breakup of molten material due only to hydrodynamic interactions. However, modeling is deficient in that breakup of material due to the impact of a jets; quenching and fragmentation of debris are not considered. A model for breakup of core material in the vessel is also not included. There are no available results of either model benchmarking/validation activities or parameter sensitivity studies.

\subsubsection{Technical Adequacy}

Models for in-vessel steam explosions, ex-vessel steam explosions, and direct containment heating are not included. Even in the absence of energetic interactions, lack of models for debris fragmentation and quenching before and after settling of the debris in a liquid pool represent significant limitations of the code with respect to modeling of severe accident phenomena. Thus, the models are incomplete and as such are incapable of describing the physical processes. Lack of models for steam explosions, direct containment heating, and debris fragmentation and quenching before or after settling of the debris in a liquid pool represents a significant limitation of the code with respect to modeling severe accident phenomena. Development of models for ex-vessel steam explosions and direct containment heating is currently planned.

\subsection{CAV Phenomenological Package Review Summary}

\subsubsection{Phenomenological Package Description}

In MELCOR, the interactions of core debris with the basemat concrete is modeled by the CAV package. CAV integrates the CORCON/MOD2 code into the MELCOR architecture.

CORCON/MOD2 models the thermal attack of core debris on concrete using a quasistatic, multilayered approach consisting of the core debris pool, the concrete cavity, and the water and/or atmosphere above the debris pool. The decay and chemical reaction energy generated within the debris pool is transferred to the concrete basemat and sidewalls, as well as the overlaying water pool and/or atmosphere.

Upon release onto the cavity basemat, the core debris is assumed to separate into layers of oxides and metals. Layer orders is determined by their relative densities (heaviest on the 
bottom). These layers consist of the dense oxide component which is rich in $\mathrm{UO}_{2}$ fuel, the metallic layer, and the light oxide layer containing mostly concrete and steel oxides. Water is allowed to exist only on the top surface.

The partition of heat transfer is via corresponding thermal resistances (upward losses are sources to $\mathrm{CVH} / \mathrm{FL}$ and HS modules). Concrete ablation and decomposition generates gases and molten oxides. These decomposition gases are reduced as a result of interactions with the debris pool metallic constituents to produce $\mathrm{H}_{2}$ and carbon monoxide gases. Both the reacted and unreacted decomposition gases eventually enter the atmosphere above the pool as sources to the $\mathrm{CVH} / \mathrm{FL}$ package.

The CAV-calculated concrete decomposition gases (rates and constituents) together with the layer temperatures are passed to the VANESA code (RN package) for calculation of fission product releases during core-concrete interactions.

\subsubsection{Qualitative Perspective}

Several deficiencies have been identified in various models included as part of the CAV package; however, the overall modeling approach appears adequate especially for dry cavity conditions.

\subsubsection{Technical Adequacy}

A prioritized summary list of Categories 4 and 5 as well as other important review findings follows:

Heat Transfer to Concrete. The present gas film flow path length in calculating heattransfer coefficient on inclined surfaces is incorrect. The approach used to calculate heat-transfer coefficients on surfaces having an angle of inclination between () and 30 degrees is not mechanistically justified. The ratio of downward to radial heat transfer to concrete has also been found to underpredict experimental data.

Pool Surface Heat Transfer. The radiative effects of aerosols must be included in the pool surface heat-transfer model to account for changes in the atmospheric opacity as a function of aerosol concentration.

Heat Transfer to Water. The modeling of thermal and hydrodynamic interactions with the overlying water pool is deficient. Models are needed to treat the potential for crust breakup, water penetration, fragmentation, quench, debris dryout, and remelt.

Debris Spreading. This is an important phenomenon, especially for BWRs with MARK-I and II containments. The current CAV model assumes an instantaneous spreading of the core debris over the entire user-specified floor area. Debris thermal interactions with metallic structural surfaces are not included. Simple spreading models are available in the literature (e.g., Moody's model, MELTSPREAD model, etc.) and should be evaluated for implementation into the MELCOR/CAV package. Any spreading model should also include potential effects of flooding and direct contact interactions with metallic surfaces.

Qxidic and Metallic Phase Diagrams. Effects of eutectic formation on debris layer melting and solidification behavior are important. Improvements in CORCON/MOD2 phase diagrams are essential. Current incompatibilities in material properties between the CAV and other MELCOR packages need to be eliminated.

Concrete Response. The present model neglects energy transfer to concrete if debris temperature is below concrete ablation temperature (nonablative attack). Transient conduction is important both during the very early and late phases of interactions.

Interlayer Mixing and Separation. The current layers separation model is not based on a mechanistic quantitative criterion (layers are assumed to form). During the initial hightemperature phase of interactions, gas release from concrete is very vigorous, leading to intermixing of oxidic and metallic constituents. Interlayer mixing criteria, along with a well-mixed debris pool model, need to be developed. 
CORCON/VANESA Consolidation. The current MELCOR integration of CORCON and VANESA is a considerable improvement over the Source Term Code Package (STCP); nevertheless, the consolidation of these modules into a unified code as currently planned for CORCON-MOD3 is desirable. This should also resolve the existing problem of duplicative but inconsistent chemistry between CORCON and VANESA codes.

\subsection{BUR Phenomenological Package Review Summary}

\subsubsection{Phenomenological Package Description}

The MELCOR combustion model is very similar to the treatment in Version 1.5 of the HECTR code (Ref. 2-5). However, the "continuous burn" option in HECTR, which is used to model combustion induced by high temperatures, is notable by its absence. Briefly, the MELCOR model calculates flammability, combustion completeness, burn time, and burn propagation to adjacent volumes using empirical formulas and a lumped parameter formulation. A summary of the approach used in each of these areas will be given below. Only deflagrations will be discussed, since detonations are not modeled.

Elammability Model. The correlations for flammability are based on experimental data. Only gas composition and the presence of igniters are considered. The user can alter the default constants used in the correlations, but control functions cannot be used.

Burn Completeness. The default burn completeness correlations are also empirical and consider only the concentrations of the various gases. The user can override the default correlations with a control function. In fact, SNL regards this an area of such high uncertainty that there is consideration being given to removing the default correlations from MELCOR entirely.

Combustion Rate. The default combustion rate model follows in a relatively straightforward way from a calculated final burn time. This in turn is computed from an empirical correlation for flame speed and a user-supplied characteristic dimension of the compartment where the burn is occurring.

Burn Propagation. Whether burns can propagate from a compartment to adjacent compartments is determined in MELCOR by logical rules. These rules are based on the combustible gas concentration in the downstream volume and the relative orientation of the two volumes.

\subsubsection{Qualitative Perspective}

Elammability Model. While adequate for many situations, the limitations of the flammability model mean that it is difficult for a user to model either auto-ignition of gases in hightemperature control volumes or the ignition of high-temperature jets leaving an inerted compartment and entering an air-bearing volume. Moreover, the documentation does not alert the user to consider these possibilities or suggest means by which to simulate the phenomena.

Burn Completeness. Based on published benchmarks of the very similar HECTR model, the MELCOR correlations will tend to overpredict burn completeness when there is a significant concentration of steam, e.g., greater than $30 \%$ by volume (Ref. 2-6). Such conditions are quite common in severe accident calculations, and overprediction of burn completeness will increase the predicted containment loads. The default model also will not model diffusion flames at igniters.

Combustion Rate. While the correlations for flame speed are stated by the documentation to be suspect if high inertant concentrations exist, the model is probably important only if very long burn times are comruted. It is not believed that this occurs frequently in practice.

Burn Propagation. A key implicit assumption in the burn propagation model is that the volume in which the burn is occurring is always well-mixed, i.e., a burn will displace both burned and unburned gases in proportion to their average concentrations in the volume (so-called "homogeneous burn" approximation). This assumption clearly introduces error, and Sandia has stated that this is considered a serious limitation by the HECTR development staff. 


\subsubsection{Technical Adequacy}

As a whole, the BUR package is considered adequate for most PRA applications. However, localized burns induced by high temperatures or the operation of igniters, which can greatly reduce the magnitude of the pressure loadings on containment, are explicitly modeled only if the user chooses to go beyond the code defaults and then only with difficulty. To a lesser degree, a concern also exists that the severity of global deflagrations occurring in the presence of significant inertant (e.g., steam) concentration is overpredicted.

With respect to the individual submodels, the flammability model is assigned to Category 2 for applications not involving high-temperature-induced burns, but for those situations that do involve such burns, the model is considered to be Category 6 . With regards to the latter, it is recognized that a skilled user can in some cases perform sensitivity analyses by using tricks, but it was judged that this is made too difficult by the current structure of the model to warran: any other assignment.

For burns caused by ordinary deflagrations, the burn completeness model is assigned to Category 2 in view of the uncertainties surrounding the effects of inertants on burn completeness; for je burning or burns at igniters the model is considered Category 3.

The combustion rate model is only a rough approximation but is considered reasonable and is assigned to Category 2. Similarly, while the well-mixed assumption does clearly introduce error into the calculation of burn propagation to adjacent volumes, this model is also judged to be reasonable and is considered Category 2 . The Committee recommends that

- the flammability/ignition limit modeling be generalized to allow at least parametric consideration of the effects of temperature, and

- the documentation be enhanced to alert the user to the need to consider the effects of diffusion flames, burning of high-temperature jets, and auto-ignition in hightemperature regions. The documentation should also note the general overprediction of burn completeness at high steam fractions.

\subsection{ESF Phenomenological Package Review Summary}

\subsubsection{Phenomenological Package Description}

The Engineered Safety Feature (ESF) package consists of two independent models. These will be discussed separately below.

Ean Cooler Model. The fan cooler model is intended to calculate the total heat removal rate and the steam condensation rate from the containment atmosphere resulting from the operation of PWR fan coolers. The MELCOR model has also been used to model BWR reactor building room coolers and would presumably be used if one was to model drywell coolers.

The model is a modified version of the model in the MARCH code (Ref. 2-7) and is quite simple. The formulation is based on correlations to fan cooler test data given in a previous revision of the Oconee Final Safety Analysis Report (FSAR) (Ref, 2-8). Separate heat-transfer coefficients are inferred from this data for sensible heat transfer and condensation. The former is constant and the latter depends only on the steam fraction.

Spray Model. The spray model is virtually identical to the model in the HECTR code (Ref 2-5). Textbook correlations are used to track the changes in temperature and mass of one or more representative spray droplets as they fall through the containment atmosphere. In practice, only one droplet size is nomally treated due to limitations in the RN package. 


\subsubsection{Qualitative Perspective}

Ean Cooler Model. The model has a number of major shortcomings. First, the heattransfer data on which the model is based have been removed from the FSAR; it is believed that the utility concluded that a mechanistic model was necessary for predicting fan cooler performance. Extrapolating design basis test data to severe accident conditions is also questionable. Fiven if the data are taken at face value, the means by which the data were transformed into a condensation heat-transfer coefficient correlation and the way that this is used in the fan cooler model are both nonphysical. As a result, the relative contributions of condensation and sensible heat removal to the total heat removal in the fan cooler are likely to be in error.

On the other hand, safety-grade fan coolers typically have far more capacity than is needed to remove decay heat. Further, in many plants the fan coolers are not safety grade and would not ordinarily be credited. For these reasons, the shortcomings of the fan cooler model are not considered especially serious for PRA work.

The use of the model for accident management work is more problematical. In recovery scenarios, expected errors in condensation rate could well impact assessments of the dangers of deinerting the containment atmosphere and calusing burns. We would also be concerned if relatively low-capacity units (room coolers and nonsafety-grade fan coolers used for normal heat loads) were thought to be important. For example, such equipment might be modeled to evaluate environmental conditions for equipment survivability assessments.

Spray Model. The most important shortcoming of the spraty model is that it cannot be used in a very high steam fraction environment, such as will occur in the primary system and, perhaps, in the drywell. Sandia has stated that the model was never intended for use in the primary system, so it is perhaps more appropriate to consider a primary system spray model as a "missing" model. The spray model also neglects radiation to droplets, which may lead to errors for short periods of time if the gas temperature is very high, e.g., if sprays are activated during or before a burn or if spray flow is small.

\subsubsection{Technical Adequacy}

Fan Cooler Model, For most PRA applications, it is judged that errors in fan cooler modeling are relatively unimportant for the reasons cited above. Thus, the model is assigned to Category 7 for PRA applications.

For recovery scenarios investigated as part of accident management work, however, expected errors in condensation rate relative to sensible heat transfer are of concern. The model is not adequate for such applications and is assigned to Category 4 . In view of other priorities, no work is recommended at this time, however.

Spray Model. For typical PRA and AM applications, the spray model is judged to be adequate for containment applications and is thus assigned to Category 1.

\subsection{Properties Phenomenological Package Review Summary}

\subsubsection{Phenomenological Package Description}

General. The MELCOR properties packages supply material property and decay heat data to the core and structural models in the rest of the code. Thermodynamic data required by the hydrodynamic models in the control volumes are supplied through the control volume thermodynamics (CV'T) package, which itself makes use of the non-condensable gas (NCG) and water properties (H2O) packages to obtain the basic data. The partitioning of the control volumes each into pool and atmosphere components is described briefly, but the assessment of this formulation is taken into account elsewhere in the section devoted to the control volume hydrodynamics (CVH) package, with which the CV'T package is closely linked. Physical properties of materials are evaluated by the materials properties (MP) package, while decay heat is calculated in the DCH package. The transfer process (TP) package controls the transfer of core 
material between pairs of packages but itself contains no physical modeling; it is noted that some modules, such as CORCON, use their own set of material data. The packages are described in the relevant sections of Refs. 2-9 and 2-10.

Control Volume Thermodynamies. In the MELCOR formulation, each control volume in the system model is composed of a pool and an atmosphere. In the 'equilibrium' option in the code, these are in thermal and mechanical equilibrium; in the 'nonequilibrium' option (usually used) only mechanical equilibrium exists. In the latter option, each part is in equilibrium model internally; it is not a true disequilibrlum model (which would support, for example, the presence of superheated steam and subcooled water droplets).

The thermodynamic properties of water (liquid or steam) are taken from the $\mathrm{H} 2 \mathrm{O}$ package, which is based on an analytic formulation of the Keenan and Keyes equation of state extended beyond $1598 \mathrm{~K}$ by JANAF data, as discussed in Ref. 2-10.

Thermodynamic properties of a range of noncondensable gases are available from the NCG package. The gases are modeled as general ideal gases, with temperature-dependent specific heats. The gases covered include nitrogen and hydrogen, while user-defined gases may also be specified. Numerical methods involve analytic fitting to temperature-dependent properties.

Material Properties. The material properties (MP) package evaluates the specific heat, melting temperature, heat of fusion, and thermal conductivity and density for fuel and structural materials such as Zircalloy and $\mathrm{UO}_{2}$, as required by the core (COR) and heat structure (HS) packages. Thermal conductivities of fluids such as water/steam and air are also available, as are binary diffusivities. User-defined materials may be specified.

Decay Heat. The DCH package provides decay heat powers for other packages, in the initial heatup, core uncovery, and melting stages of a postulated accident up to and including fission product release. It does not treat fission product transport or chemical interactions. Whole core options are available along with an elemental group treatment based on ORIGEN calculations. In the latter option, 29 elemental groups are divided among 13 chemical classes; a major assumption is that daughter isotopes are transported along with their parents. The whole core options include summation of decay heat data from ORIGEN calculations and use of the ANS-5.11979 standard. There is a considerable amount of flexibility for the user both in selection of model options and in the ability to carry out sensitivity studies.

Transfer Process Package. The transfer process package provides the means to transfer the mass and enthalpy of materials among the COR, FDI, CAV, and RN packages. The data are managed through a central data base that accepts and distributes the information as required. The identities of materials may change across the package; for sxample, steel may be broken down into its constituent elements. Energy errors can occur if the equations of state for a material differ in different modules (as, for example, between the COR and CORCON models); these are noted in the code output.

\subsubsection{Qualitative Perspective}

General. The modeling in the properties package follows well-established principles and contains appropriate detail with respect to the packages in the rest of the code for which data are supplied. The treatment is for the most part state-of-the-art and the package as a whole is judged to be near the end of its development.

Control Volume Thermodrnamics. In the thermal-hydrallic area, the data base and methods used in the water properties package are standard and well established. The analytic formulation used here ensures good consistency but is computationally expensive. In the NCG package, while the origin of some of the preset data is obscure, the principles of the modeling are again judged sound.

Material Properties. Turning to the materials area, there appear to be no serious errors in the thermal properties of major reactor materials. The principal needs are for some tidying-up of the documentation, identifying clearly the sources of the data, and checking the consistency of user-specified data. Deficiencies in the calculation of gas mixture properties identified in Version 1.8.() of the code were rectified in Version 1.8.1. More sensitivity 
coefficients would be useful, e.g. for binary diffusivities. If eutectic reactions are included in the COR package, properties for eutectic mixtures will be needed from MP; data in this area are sparse.

Decay Heat. In the decay heal area, the user can choose among a number of physically reasonable, state-of-the-art options with the facility to carry out sensitivity studies. The level of detail is appropriate for the application.

Transfer Process Package. The treatment in this area is judged adequale. The proposed move to make the transfer processes more transparent to the user is welcomed.

\subsubsection{Technical Adequacy}

There are no major technicul adequacy issues (Category 4 or 5 findings) or major missing models associated with the properties package since as a whole it is judged satisfactory. In the long term, it would be desirable to have a common set of material properties in use throughout the code. The few minor deficiencies in individual areas are unlikely to give rise to significant errors in a PRA context either in the calculation of the time to containment failure or in the estimation of the source term.

\section{REFERENCES}

2-1. M. R. Kuhlman, D. J. Lehmicke, and R. O. Meyer, "CORSOR User's Munual," Battelle Columbus Laboratories report BMI-2122 (NUREG/CR-4173) (March 1985).

2-2. J. C. Helton, R. L. Iman, C. D. Leigh, and J, D. Johnson, "Uncertainty and Sensitivity Analysis of an Upper Plenum Test Problem for the MAEROS Aerosol Model," Aerosicol Science and Technology 7, p. 199-216(1987).

2-3. J. C. Helton, R. L. Iman, J. D. Johnson, and C. D. Leigh, "Uncertainty and Sensitivily Analysis of a Dry Containment Test Problem for the MAEROS Aerosol Model," Nuclear Science and Engineering 102, p. 22-42 (1989).

2-4. J. C. Helton, R. L. Iman, J. D. Johnson, and C. D. Leigh, "Uncertainty and Sensitivity Analysis of a Model for Multicomponent Aerosol Dynamics," Nuclear Technology 73, p. 32()-342 (June 1986).

2-5. S. E. Dingman, A. L. Camp, C. C. Wong, D. B. King, and R. D. Gasser, "HEC'TR Version 1.5 User's Manual," Siandia National Laboratories report SAND86-()1()1 (NUREG/CR-4507) (February 1986).

2-6. C. C. Wong, "HECIR Analyses of the Nevada Test Site (NTS Premixed Combustion Experiments," Sandia National Laboratories report NUREG/CR-4916 (November 1988).

2-7. R. O. Wooton, P. Cybulskis, and S. F. Quayle, "MARCH 2. (Meltdown Accident Response Characteristics) Code Description and User's Manual," Battelle Columbus Laboratories report BMI-2115 (NUREG/CR-3988) (September 1984).

2-8. Duke Power Company, "Oconee Nuclear Station Units 1, 2, and 3: Final Safety Analysis Report" (last version).

2-9. R. M. Summers, R. K. Cole, Jr., E. A. Boucheron, M. K. Carmel, S. E. Dingman, I. E. Kelly, "MELCOR 1.8.(): A Computer Code for Severe Nuclear Reactor Accident Source Term and Risk Assessment Analyses," Sandia National Laboratories report SAND9()-(0.364 (NUREG/CR-55.31) (January 1991).

2-10. R. M. Summers, "MELCOR Primer, Version 1.8.()," Sandia National Laboratories unnumbered report (February 1\%, 1989). 


\section{FINDINGS RELATIVE TO CODE OBJECTIVES}

\subsection{Identification of Code Objectives}

As identifted by the NRC in Ref. 3-1, the code design objectives are as follows.

Objective 1: MELCOR should appropriately and consistently model phenomena essential to the description of severe light water reactor (LWR) accidents and should allow representation by the user of plant components and systems essential to the manngement of severe LWR accidents, including effects of equipment response and operator actions on accident progression and mitigation strategies.

Objective 2: MELCOR should provide rensonable predictions of the progression and consequences of severe accidents.

Objective 3: MELCOR should permit estimates of the uncertainties associated with its predictions of severe core damage accidents to be made without requiring modifications to the code.

Objective 4: Models incorporated in MELCOR should be in adecunte detail to address important phenomenology but permit a practical running time for accident sejuence analysis.

Objective 5: MELCOR should be applicuble for severe accident studies and related plant applicutions for both PWRs and BWRs.

Objective 6: MELCOR should be portable, maintainable, and have a structure that facilitates incorporation of new or alternative phenomenological models.

Access to a statement of code objectives was an important and necessary part of the Committee review process. However, many of the terms appearing in the code objective statements are, in themselves, open to a vilriety of interpretations. Examples of such terms are "appropriately and consistently," "phenomena essential to the description of severe light water reactor accidents," and "realsonable predictions." Thus, the Committee found it necessary to prepare definitions of these terms so that the individual Committee members could prepare findings relative to a set of standard definitions. As the Committee findings regarding each code objective are presented in the following, the appropriate Committee definitions that further interpret and develop the objective statements will be introduced.

\subsection{Objective 1}

\subsubsection{Statement}

MELCOR should appropriately and consistently model phenomena essential to the description of severe LWR accidents and should allow representation by the user of plant components and systems essential to the management of severe LWR accidents, including effects of eyuipment response and operator actions on accident progression and mitigation strategies.

\subsubsection{Supporting Definitions}

Objective 1 contains three sublevel objectives; some are closely related to the bottom-up review activities of the Committee while others are related to the top-down or integrated features of MELCOR.

The Commiltee interpreted the first sublevel term "appropriately" to be synonym for "technical adeguacy." As discussed in Chapter 1, a finding that a model is technically adeguate 
(appropriate) is based upon (1) model pedigree, (2) model upplicability, and (3) model fïdelity 'The definitions from Chapter 1 are repeated here for convenience.

Model pedigree is based upon the model origin; the basis for the model including physical reasonableness, assumptions, restrictions, simplifications; the original application of the model and its relationship to the MELCOR application; and the experimental or other dala supporting the model.

Model aoplicability bullds on the concept of pedigree, but, in addition, is based upon the relationship of the model to the dominant phenomena being modeled and the existence of and acceptable justification if the model is used beyond the original pedigree.

Model fidelity builds on the concepts of pedigree and applicability, but, in addition, is based upon the ability to predict dominant phenomena with acceptable accuracy. Thus, the key element of fidelity is benchmarking of the model against other standards. The highest level standards are exact solutions, followed in descending order of desirability by prototypical data, other data, code-to-code comparisons, and expert opinion.

Because these definitions are focused on the models and correlations in MELCOR, findings of appropriateness are most closely related to the bottom-up part of the Committee review.

The Committee interpreted the second sublevel term "consistently" to relate to the topdown review of MELCOR. 'The code is consistent if code parameters and physics have the same definition and usuge throughout the code. A parameter calculated in one code package must have the same physical definition in other packages to which it is transferred if the phenomena is modeled consistently. Thus, individual phenomenological packages should not be based upon contridictory assumptions.

The third sublevel objective focuses on the code's capability to represent the plant components, systems, and operator actions features in the plants of interest, BWRs, and PWRs.

\subsubsection{Findings for Objective 1, Sublevel 1: Appropriate ('Technically Adequate) Modeling of Phenomena Essential to the Description of Severe LWR Accidents}

Detailed or bottom-up review findings for each phenomenological package are provided in Appendices D through K. Summaries of the hottom-up findings regarding the technical adecuacy of the models and correlations contained in each MELCOR phenomenological package are provided in Chapter 2. For some of the phenomenological packages, deficiencies in the modeling of phenomena essential to the description of severe light water accidents were identified; all such deficiencies are described in the appropriate appendix. The Chapter 2 summaries of each phenomenological package list the three or four most important model deficiencies for that phenomenological palckage in the judgment of the package reviewers (see 'able 1-II).

However, the Committee felt that it was necessary to judge, in some equitable and consistent manner, the relative importance of each deficiency and thereby provide recommendations regarding the order in which deficient models should be corrected. In assigning priorities, the Committee focused on the PRA application using as figures of merit the relative importance of the potential impact of the deficiency on (1) the time of containment failure, and (2) the magnitude of lission product release to the environment. Relative to these two figures of merit, the Committee evaluated eiach of the deficient models listed in the Chapter 2 summaries and assigned each model to one of three categories: very important, important, and less important. The Committee recommends that those models assigned to the "very important" category be corrected as soon as possible. Commillee reconmendations regarding these models are carried forward to Chapter 6, Recommendations. The Committee recommends that deficient models identified as "important" or "less important" be corrected as resources permit. Committee findings regarding 
the importance of identified model deficiencies within the MELCOR phenomenological packages are provided in the following sections.

3.2.3.1. Deficient Models for Which Coryetion is Very Important. An evaluation should be made to determine whether the water condensation/evaporation model used in the CVH package is implemented adequately, Currently, condensation is treated independently in the CVH package from the calculations of aerosol particle growth and deposition in the RN package. The condensation-related growth of aerosols is in competition with wall surfaces for uvailable water and hence the two processes are not independent processes. Because the size change for particles (droplets) as a result of condensation may have an overriding effect on aerosol deposition rates, the correct allocation of condensing water is a controlling factor. Therefore, the acceptability of the current model should be demonstrated (see Appendix G for further details).

Improvements in the oxidic and metallic phase diagrams used in the CAV package are needed because the effects of eutectic formation on debris layer melting and solidification behavior are important. Current incompatibilities in material properties packages between CAV and other MELCOR packages need to be eliminated. A planned consolidation of CORCON and VANESA into a unified code should resolve existing problems of duplicative but inconsistent chemistry between these two codes. It is the Committee's understanding that improvements leading to CORCON-MOD3, currently in progress, are intended to resolve these modeling problems as well as other shortcomings identified by the Committee assessed as having lesser priority (for details about the CAV package, see Appendix H).

The model for condensation in containment (mass transfer) should be revised. The modeling of condensation in the presence of noncondensables is physically incorrect as film thermal resistance is neglected. With the existing model, steam condensation rates predicted for conditions when the thermal resistance of the structure on which condensation occurs does not dominate the total resistance will be in serious error. The model used for condensation in the presence of noncondensables is seriously flawed (see Appendix E for further details).

The aerosol pool scrubbing model is largely derived from previously available models but assumes spherical bubble shapes while correcting for bubble flattening by using coefficients derived from comparisons to other models. Impaction of particles from steam/air jets entering the pool would be expected to provide significant deposition for larger particles but the effect is ignored in the current model. Decontamination factors computed with the current model are quite low in comparison with other models and the existing data base (see Appendix $G$ for further details).

3.2.3.2. Deficient Models for Which Correction is Important. Condensation and evaporation of fission product species are treated as a straightforward mass-transfer process based on an analogy to heat transfer. The mass-transfer coefficients are imported from the HS package. The analysis is adequate but could be improved by allowing for dilution effects which reduce the vapor pressure of deposits (see Appendix $G$ for further details).

The present modeling of the pool-side boundary layer and its impact on condensation at pool/atmosphere interface is incorrect and should be corrected. Overprediction of condensation rates can be expected for conditions such as a steam atmosphere overlying a large quiescent water pool (see Appendix D for further details).

3.2.3.3. Deficient Models for Which Correction is Less Important. The fission product release model is predicted with the CORSOR/CORSOR-M code which is based on older data and is of limited versatility. Newer data and correlations based on these data are now becoming available, which should model the time dependence of the releases more accurately. Extension of the release models to slumped or pooled fuel may be possible with correlations based upon newer datia. Release from core-concrete interactions is treated by a very early version of VANESA known to have errors and limitations and upgrade to a newer version is recommended (see Appendix (i for further details).

At high cemperatures, radiation exchange between structures can be very important with respect to the determination of the heatup rate of the reactor cooling system. Currently, the user is 
required to provide shape factors. Models or correlations for shape factors should be added to the code as a user option (see Appendix E for further details).

\subsubsection{Findings for Objective 1, Sublevel 2: Consistency Between Models and the Phenomenological Packages}

The objective of consistent treatment of important phenomena or plant features has been central to the MELCOR development effort since its beginning. For example, Ref, 3-2 (p, xi) documents an attempt to "gather the information from the experts in the various fields that will be needed by the MELCOR code developers to define a consistent set of models to be used" in MELCOR. Initially, there was also a clear development philosophy. Before beginning code development, SNL staff stated the following (Ref. 3-2, p. 1.1-22): "It should be recognized that MELCOR is not intended to be designed as a tool for the resolution of such uncertainties (e.g., modeling of ATWS sequences, multiple SGTRs, 'feed and bleed' capability, late emergency coolant injection onto a partially damaged core, and determination of success criteria). Existing best-estimate codes such as TRAC and RELAP5 as well as future detailed phenomenological codes such as SCDAP and MELPROG are more appropriate tools for resolving such uncertainties. In fact, the detailed neutronic and thermal-hydraulic capabilities associated with such codes would conflict with the objective that MELCOR be a fast-running code with proven models. It is recognized that less detailed modeling may impose certain limitations on MELCOR."

However, the development philosophy has changed through the intervening years. The recently issued MELCOR summary manual states the following (Ref. 3-6, p. 1-5): "Initially, the developers and the NRC believed that relatively simple parametric models would be adequate in most areas. However, the initial assessment of phenomenology and modeling indicated that this would not be acceptable to the reactor safety community. In fact, each contributing expert tended to recommend near state-of-the-art modeling. Therefore, most MELCOR models are mechanistic, with capabilities approaching those of the most detailed codes of a few years ago."

This switch in the MELCOR development philosophy has led to some inconsistencies within MELCOR related to its modeling of phenomena and physical features. At least some of these inconsistencies arose when existing codes such as CORCON were incorporated into MELCOR. It is probably true that the Committee has not identified all such inconsistencies. It has, however, identified enough inconsistencies to determine that achievement of the objective of consistency has not been completely fulfilled. Examples of several inconsistencies identified by the Committee are as follows: (1) relocated materials, while remaining in the $\mathrm{RV}$ lower head, are characterized by a set of material properties; once these materials have exited the vessel, the CORCON phenomenological package takes over and a new set of material properties within CORCON are used; (2) CORCON assumes stratified solid materials on concrete while VANESA does not assume stratified materials; (3) fission products settling on a dry floor retain their position even after the subsequent addition of water while fission products settling into a pool will flow with the water; (4) the treatment of debris/water interactions in the lower plenum and in the containment are different with large heat-transfer rates to particulate debris in the lower plenum and negligible heat transfer in the containment; and (5) debris particle size is a user input in the vessel, ex-vessel calculations invoking FDI are based upon a fragmentation model, and CORCON assumes an impenetrable mass.

To provide perspective, the Committee notes that considerable progress has been made in the area of consistency when MELCOR is compared to STCP.

\subsubsection{Findings for Objective 1, Sublevel 3: Code Capability to Represent the Plant Components, Systems, and Operator Action Features}

This objective addresses the capability of the MELCOR code to represent the physical and operational features of LWRs. This objective lists the physical and operational features to be modeled; they are the plant hardware, the plant systems, and operator actions. Examples of the plant hardware are the reactor vessel, reactor core, piping, valves, steam generators, pressurizers, accumulators, containment, etc. Examples of the plant systems are the emergency core cooling 
system (ECCS), the residual heat removal system (RHRS), and the plant protection system (PPS), These systems may consist of a combination of hardware, electronics, and software. Examples of operator actions are throttling flows, closing valves, and tripping the reactor.

The Committee has concluded that the MELCOR code satisfies the objective of providing a code capable of adequately representing the physical and operational features of LWRs components, systems, and operator actions with only limited exceptions, notably ice condenser plants.

Components are modeled using a generic building-block approach. The hydrodynamic characteristics of the reactor are modeled using control volumes and connecting paths. Plant modeling involves creating input to define the interconnection of a number of control volumes by flow paths. Generalized heat structures are available to model solid materials such as pressure vessel internals and walls, containment structures and walls, fuel rods with nuclear or electrical heating, steam generator tubes, piping walls, etc. Those materials subject to degradation and material relocation processes must be specifically identified at the time the MELCOR input deck is developed and represented in COR rather than HS. Where possible, MELCOR also uses a generic builuiv-block approach to modeling systems through the use of control volumes, flow paths, heat structures, and control functions. The capability to develop component models has been previously discussed. However, the systems frequently require control elements. MELCOR provides a broad capability, through its control function modeling, to define arbitrary functions of variables and make these control functions available to the individual phenomenological packages in MELCOR for use in controlling various models. Thus, the control function capability can be used to simulate the operation of various electronic and software controls in L.WRs.

The control function capability can also be used to model virtually all operator actions that can be defined.

\subsection{Objective 2}

\subsubsection{Statement}

MELCOR should provide reasonable predictions of the progression and consequences of severe accidents.

\subsubsection{Supporting Definitions}

The thrust of this objective is the code's capability to calculate the integrated phenomena of severe accident sequences with a degree of accuracy identified as "reasonable." Committee findings regarding Objective 2 naturally fall in the category of top-down or integrated code capabilities.

Any Committee attempt to prepare findings of technical adequacy relative to Objective 2 must be based upon a realistic expectation of the state of the art with respect to knowledge of severe accident phenomena and modeling of these phenomena. The Committee considered this issue as reported in Section 1.3, Item 7. That perspective, related to "Knowledge of Physical Processes," is repeated here for convenience.

The Committee acknowledges that the current level of scientific knowledge regarding severe accident processes varies. The physics of some physical processes are well understood while the physics of other physical processes are partially or poorly understood, i.e., severe accidents are complex. follow uncertain paths, and include some phenomena that are not well characterized at present. Given the reality that the physics of some severe accident phenomena remain uncertain at present, the Committee cannot expect MELCOR to accurately model that which the scientific community has not been able to adequately characterize. The MELCOR code should, therefore, permit the 
consideration of uncertain physical processes by other means (e.g., sensitivity studies).

Given this caveat, the Committee has prepared findings relative to Objective 2 in five areas: (1) code numerics, (2) comparison to integral benchimarks, (3) code-to-code comparisons, (4) sensitivity analyses, and (5) the reasoned judgments of Committee members.

\subsubsection{Findings for Objective 2}

In the following, Committee findings regarding each of the five areas previously identified are provided.

3.3.3.1. Code Numerics.' In a large code having the architecture (phenomenological package construction) of MELCOR, an evaluation of the overall code numerics is problematical. Difficulties in evaluating root causes may arise from several sources such as, for example, (1) numeric techniques that vary from package to package, (2) explicit coupling of the packages at every time step, (3) the difficulty of discerning whether instabilities arise from natural phenomena or the numerics, (4) the lack of documented test cases that isolate and describe the performance of the code numerics, and (5) the unavailability of extensive code sensitivity studies.

The Committee feels that code numerics are a primary concern regarding the technical adequacy of the code. During the course of the MELCOR Peer Review, the results of several timestep sensitivity studies were made available to the Committee. Madni (Ref. 3-3) reports that the selection of the maximum time step, $\Delta t_{\text {max }}$, and its impact on the calculational behavior of MELCOR "is an area of lingering uncertainty in the use of MELCOR." The impact of varying the maximum time-step specification was explored for a station blackout transient in Peach Bottom using MELCOR Version $1.8 B C$ and $\Delta t_{\text {max }}$ values of $10 \mathrm{~s}$ (reference calculation), $5 \mathrm{~s}$, and $3 \mathrm{~s}$. Madni's results do not display a trend towards convergence. Rather, the largest deviations relative to the reference time step were associated with $\Delta t_{\max }=5 \mathrm{~s}$ and the deviations were smaller for the 3-s time step. Thus, a trend towards convergence was not observed.

Khatib-Rahbar (see presentation 3-8 of Ref. 3-4) discussed similar time-step sensitivity results and reported "reductions in time step do not necessarily lead to a converged solution" although "bottom line source term results /are) generally less sensitive to time step sizes." The calculations were performed for a BWR with a Mark-I containment using $\Delta \mathrm{t}_{\text {max }}$ values of 5,3 , and $1 \mathrm{~s}$. The reported times of lower vessel head penetration failures (in MELCOR model ring 1) were calculated as $18,8(0), 10,700$, and $19,700 \mathrm{~s}$ for $\Delta t_{\max }$ values of 5,3 , and $1 \mathrm{~s}$, respectively. Again, no trend to convergence with reduced maximum time step was observed. Related results were reported by the Swiss Federal Nuclear Safety Inspectorate in Ref. 3-5.

SNL notes that several users have noted sensitivities to time-step size in some calculations and states "Some of these sensitivities are believed to be unavoidable because of the threshold nature of some models and the complex interplay between competing processes, but others are not yet fully understood" (Ref. 3-6, p. 6-4). One example of these interactions is detailed by Hyman (Ref. 3-7). Hyman observed that the instabilities were related to the interlayer heat-transfer algorithms HTRLAY and INTEMP for the in-pedestal CORCON calculations. He also noted that the numerical problems might be related to either inadequate modeling of the interlayer physics or a numerical solution scheme that is not sufficiently stable. It should be recognized that this observation applied to the stand-alone CORCON code and may not be applicable to CORCON as implemented in MELCOR.

Similar numerical sensitivities have been encountered with the integral severe accident code MAAP. In a recent presentation (Ref. 3-8), the Electrical Power Research Institute (EPRI) noted sensitivities to small changes in input parameters that produced unacceptably large and "chaotic" changes in, for example, containment failure time and core debris distribution between the cavity and lower compartment. EPRI also noted poor reproducibility of results from one computer to another and between single- and double-precision calculations. EPRI noted that these problems were observed in the PWR version of the code but were not identified in the BWR version of the code. All these problems have also been observed with MELCOR. EPRI reported 
good progress in solving these problems but emphasized that a large effort was required to improve the numerical performance of the MAAP code.

The Committee has concluded that an improved understanding of the time-step sensitivities is important and should be considered to be a high-priority activity. As Madni stated, "Note that [the best] $\Delta t$ is problem dependent and a $\Delta t_{\max }$ found most appropriate for benchmarking MELCOR with an integral experiment will not, in general, be the most appropriate for a full-plant simulation. Again, the most appropriate $\Delta t_{\max }$ can vary from one plant simulation to another, from one sequence to another, and within a sequence, from one stage of the transient to another." Given this circumstance, the Committee concluded that it is difficult to have the requisite level of confidence in the outcomes calculated for a given MELCOR application. In addition, such seemingly random behavior severely compromises the ability of the code to perform meaningful sensitivity analyses. The Committee has concluded that an improved understanding of the sensitivities is important and that correction of the MELCOR numerics problems should be considered to be a high-priority activity.

3.3.3.2 Comparison to Integral Benchmarks. As previously described, a complete assessment of the technical adequacy of an integral code considers both the component parts (bottom-up review) and the adequacy of the integrated coded package (top-down review). The detailed or bottom-up review focuses on the pedigree, applicability, and fidelity of the individual models and correlations. The Committee emphasizes the importance of having technically adequate detailed models and correlations. Thus, the importance of having technically adequate detailed models cannot be overemphasized.

If the detailed models and correlations are technically adequate (i.e., have acceptable pedigrees, are applicable, and have acceptable fidelity), the next step is to ensure that the individual models and correlations are successfully integrated into the overall code architecture and numerical solution scheme. It is within this context (i.e., technically adequate building blocks and appropriate integration of the individual models and correlations within the code architecture and numerical solution scheme) that the comparison of code calculations to semi-integral or integral benchmarks assumes its proper role. Thus, the capability of the code to deliver accurate integral results is the ultimate measure of technical adequacy given the complex manner in which the numerous individual models and correlations interact. Within this document, the term integral benchmark is used to describe all experiments having an integrated character including those in which a subset of the total phenomena expected in a severe accident is simulated.

Before turning to an evaluation of the integral benchmarks performed with MELCOR, several points should be considered. First, the number of integrated severe accident experiments is limited. Certainly, the severe accident at Three Mile Island (TMI-2), although unplanned, falls into this category. The LOFT FP-2 experiment is another example. The remaining integral experiments usually consider only a portion of an integrated accident sequence. Second, all benchmarks are not repeated for each released code version. Thus, the reported integral benchmarks have often been performed with earlier versions of the code. That is certainly the case with MELCOR Version 1.8.1, July 1991. If significant code revisions have been made between the time of the integral benchmark calculation and the assessment of the current code version, it is not clear how much credit to allow for an integral benchmark performed with the earlier released version of the code. Third, because MELCOR is still under active development and there are so few integral benchmarks, it is likely that the existing integral benchmarks have been extensively used in the development process.

TMI-2. MELCOR was used by SNL staff to analyze the TMI-2 accident in a standard problem assessment activity. The benchmarking activity continued over an extended period of time and is appropriately described as developmental assessment. All integral phenomena assessed were in-vessel. For consistency of the standard problem benchmarking activity, the TMI-2 transient was divided into four phases. Phase 1 covered the thermal-hydraulic transient from () to $100 \mathrm{~min}$. Phase 2 covered the boildown and early core degradation from 100 to $174 \mathrm{~min}$. Phase 3 covered the pump transient and debris heatup occurring between 174 and 224 min. Phase 4 covered the period of molten core debris relocation from 224 to 230 min. Originally, MELCOR Version 1.7.1 was used to analyze the first two phases of the TMI-2 accident (Ref. 3-9). With the 
release of Version 1.8, the first two phases were recalculated. The Phase 1 calculation was successfully run but difficulties were encountered with the Phase 2 calculation, which yielded different results than those obtained with Version 1.7.1. SNL staff then developed a more coarsely noded model that would run more rapidly and permit easier diagnosis of model interactions.

It seems evident that the TMI-2 standard problem was a valuable effort. It also seems evident that the effort was less closely related to benchmarking and more closely related to model development. Several of the modeling challenges related to the analysis of Phases 3 and 4 were provided in presentation 1-19 of Ref. 3-10. They were the lack of a core shattering and debris formation model, the lack of an associated hydrogen production model, debris heat transfer models that were too simple, no radial core material relocation, and the availability of only a gross failure specification capability for the core support plate. Answers to Committee questions during presentation 1-19 of Ref. 3-10 clearly indicated that the ability to adjust sensitivity parameters to obtain improved comparisons with data was an important factor. In some cases, it appears parametric changes were necessary because of inadequate models, e.g., the incorrect treatment of the effects of noncondensable gases on condensation rate in the steam generators. Many important modeling insights were gained from this effort which have found their way into subsequent code improvement efforts. However, although all Committee members agree that the TMI-2 effort produced important code-related information, the developmental assessment character of this effort is also noted because significant noding and sensitivity parameter adjustments were necessary during the course of the activity.

PHEBUS Severe Fuel Damage (SFD) Test B9t, MELCOR was used by SNL staff to calculate the core degradation phenomena of the PHEBUS SFD experiment, B9+, which has been selected as International Standard Problem 28 or ISP28 (Ref. 3-11). MELCOR Version 1.8EA, a developmental version of the 1.8.0 code released at a later date, was used. In addition, special code modifications were required to model the PHEBUS fuel bundle configuration as discussed in Ref. 3-11. All integral phenomena assessed were in-vessel. Several calculations were performed. The results calculated with the initial input model were not sufficiently close to the measured results. Several sensitivity studies were performed and input values changed. All changes were stated to be within assessed uncertainties. Several of the changes were (1) higher thermal conductivity of the porous $\mathrm{ZrO}_{2}$ shroud, (2) increased inlet steam flow rate of $5 \%,(3)$ decreased core fission power of $5 \%,(4)$ use of a radiation view factors of 1.0 between radial rings (higher than used in full LWR core simulations), and (5) increasing the convective heat-transfer coefficient by $50 \%$, which was justified by noting that the uncertainty of the input hydraulic diameters. A major departure of the calculated and measured bundle temperatures after $8370 \mathrm{~s}$ during the helium injection phase of the test was traced to the specified mixture material properties and the cladding emissivities used in the calculation. Overall, the calculation of material temperatures were found to be in reasonable agreement with data until the helium injection phase. The postulated reasons for disagreement during the helium injection phase has been previously discussed. Results of the bundle degradation calculations are presented but discussions of their comparison to test results are limited. It seems evident that so few integral benchmarks have been completed to date that the learning curve associated with each benchmark activity is high. Reference 3-11, for example, discusses lessons learned about user guidelines, modeling uncertainties, code errors uncovered, modeling inconsistencies, and missing models. In doing so, the value of integral assessment activities is clearly demonstrated.

Power Burst Facility (PBF) SFD Test 1-1. MELCOR Version 1.7.1 was used by Madni of Brookhaven National Laboratory (BNL) to simulate PBF SFD test 1-1 (Ref. 3-12). SFD test 1-1 was designed to simulate the heatup and resulting fuel damage in the upper half of a PWR core following a small-break loss-of-coolant accident (LOCA) at a time when the core is approximately $75 \%$ uncovered. All integral phenomena assessed were in-vessel. The MELCOR calculated results were compared to measured values of the two-phase test vessel water level, power transferred to the bypass coolant, cladding temperatures at several axial levels, a fuel temperature, average in-vessel steam temperatures, and integral hydrogen production. Madni noted the several sources of uncertainty within the test, but he concluded "the calculated results showed good overall agreement with test data." He also reported that "fission product release is 
the only result that is substantially overpredicted" and concluded that the CORSOR and CORSOR$M$ models in MELCOR are not appropriate for the trace irradiated fuel used in SFT test 1-1. During the test, cladding oxidation and melting, shroud inner liner failure, fuel liquefaction, and material relocation occurred. No information regarding the MELCOR simulation of melting and material relocation phenomena were presented.

Power Burst Facility (PBF) SED Test 1-4. MELCOR Version 1.8 was used by Madni to simulate PBF SFD test 1-4 (Ref. 3-13). SFD test 1-4 was designed to investigate fuel rod and core response and the release of fission products and hydrogen during degraded core accidents. All integral phenomena assessed were in-vessel. The MELCOR calculated results were compared to measured bundle water level, power transferred to the bypass coolant, cladding temperatures, shroud inner liner temperatures, hydrogen release, and fission product release. Madni notes that there were many sources of uncertainties in the performance of the tests and cites the failure of the shroud inner liner, thermocouple failures, and measurement uncertainties in hydrogen generation, bundle liquid level, and fission product release. He also notes that there are several model uncertainties and simplifications in MELCOR. Among these are lack of a clad ballooning model, use of a default temperature as the clad rupture limit, and lack of a model for eutectic (U-Zr-O) formation. Despite both the test and modeling uncertainties, Madni concludes that "in general, MELCOR Version 1.8 calculations have represented the bundle behavior during the test reasonably well, showing the same trends as . . the measured data." During the test, cladding oxidation and melting, shroud inner liner failure, fuel liquefaction, and material relocation occurred. No information regarding the MELCOR simulation of melting and material relocation phonomena was presented.

ELHT-2. MELCOR Version 1.8 DN was used by BNL staff to simulate Full-Length High-Temperature (FLHT) facility experiment 2 (Ref. 3-14). All integral phenomena assessed were in-vessel. The FLHT-2 test was performed by Pacific Northwest Laboratory in the National Research Universal Reactor at Chalk River, Ontario, Canada. The FLHT-2 test bundle consisted of 12 full-length unirradiated fuel rods typical of a PWR design and was enclosed in an insulated Zircalloy shroud. The authors note the test uncertainties as including fission power, heat transfer to the bypass, hydrogen generation, licuid level in the bundle, and inlet water flow rate. They also discussed several model uncertainties and simplifications in MELCOR. However, the authors concluded, "In general, MELCOR version 1.8 DN calculations have represented the bundlebehavior during the test reasonably well, showing similar trends to measured data."

Several differences between the calculated results and experimental data were identified. MELCOR underpredicts the sharp temperature rise resulting from accelerated Zircalloy oxidation and predicts the fuel temperature peak later than observed. The factors identified as contributing to these discrepancies are partially attributed to the lack of a clad ballooning model, the absence of oxidation on the inside of the clad, and the treatment of the shroud Zircalloy inner liner as a heat structure, which is not currently allowed to oxidize in MELCOR. For this test, the calculated end state is compared to the state identified from posttest examinations of the test bundle. No noticeable blockage was seen during the postirradiation examination of the FLHT-2 bundle; the MELCOR calculation predicted the same result.

Battelle Model Containment (BMC) E2 Experiment. Phase 1. MELCOR, version $1.8 \mathrm{BC}$ was used by Robertson of the United Kingdom Atomic Energy Authority, AEA Technology, to simulate Phase I of BMC test F2 (Refs. 3-15 and 3-16). The BMC facility is a 1:4 linear scale model of the Biblis 'B' PWR containment. The containment has internal concrete structures that subdivide the containment volume into rooms. During Phase I of BMC test F2, the pressure in the containment was increased step-wise by controlling the injection of steam into one of the containment compartments. The main characteristic of the Phase I test was the establishment of a horizontal steam/air interface in the upper dome of the facility which moved down with time to the lower parts of the facility as more steam was added. All integral phenomena assessed were exvessel and in-containment. Robertson reported that "MELCOR has been shown to be capable of performing multi-volume containment thermal-hydraulic calculations. Furthermore, notwithstanding the sensitivities which have been observed and discussed, the code has been shown to be able to reproduce the observed stratification in certain thermal-hydraulic parameters such as atmosphere temperature." Robertson also observed that the time-step choice was an 
important parameter in the BMC F2 Phase I experiments. Too large a time step resulted in numerical instabilities, which led to mixing of the constituents of connection cells, thus predicting incorrect results. For the calculations for Phase 1 of the BMC-F2 experiment, a maximum time step of about $2 \mathrm{~s}$ eliminated the numerical instability. Robertson concluded that the algorithm used in the code for time step choice is not adequate and should be investigated further.

HDR Tests 31.4 and 31.5. MELCOR Version 1.7.1 was used by SNL staff to simulate ISP-23 and the hydrogen mixing standard problem following ISP-23. The containment used in this test is that of the decommissioned HDR reactor facility near Frankfurt, Germany. The HDR containment is enclosed by a cylindrical steel shell with an overall height of $60 \mathrm{~m}$ and a diameter of $20 \mathrm{~m}$. An outer concrete containment surrounds the steel shell leaving an annular space between the primary and secondary containments. The primary containment is subdivided by concrete walls into 62 subcompartments containing a large number of internal metallic structures. The HDR tests 31.4 and 31.5 calculations were recently performed "blind" by SNL staff (Ref. 317). Subsequent posttest calculations of ISP-23 are also reported by Martinez in Ref. 3-17. Martinez concluded that the ". . ability of MELCOR to calculate the thermal-hydraulic behavior of a very large multi-compartment containment for a large pipe rupture simulation has been demonstrated."

HDR Test V44. MELCOR Version 1.6 was used by SNL staff to simulate the HDR experiment V44. Experiment V44 is one of a series of six water and steam blowdown experiments conducted to simulate full-scale LOCAs. Because this benchmark was performed with a MELCOR code now dated by several subsequent releases, the applicability of this assessment to the current code is problematical. However, the results of this assessment activity were factored into subsequent development activities. In summarizing this assessment activity, Shaffer (Refs. 3-18 and 3-19) reported that MELCOR predicted the peak containment pressure to be about $24 \%$ higher than measured with the longer term calculated pressures in good agreement with the measured pressures. Shaffer suggested that MELCOR's heat-transfer correlations, which in Version 1.6 were consistent with then accepted contaimment blowdown coefficient correlations, calculated heattransfer coefficients that were too small to accurately predict the dynamic containment behavior during blowdown. It could also be that steady-state correlations were used to model a dynamic process. All integral phenomena assessed during this assessment were ex-vessel and incontainment.

Qther Tests. Several additional assessments having some integral characteristics are reported in Refs. 3-18 and 3-19. However, these tests were performed with earlier versions of MELCOR (1.5 to 1.()) and are not discussed further here. The Committee also notes the existence of a considerable body of assessment information for stand-alone codes such as CORCON that have been incorporated either partially or wholly inte MELCOR. The Committee found it difficult to assimilate such assessment information given yuestions about code versions and how the parent code may differ from the version incorporated into MELCOR.

Summary Comments. By any measure, the MELCOR integral benchmarking effort is extremely small. The limited scope of the MELCOR validation effort is apparent if one notes that more than 10() assessments of integral thermal-hydraulic tests were performed with various versions of the TRAC-PF1 code. This disparity becomes even more evident when one considers that the thermal-hydraulic phenomena against which TRAC-PF1 was assessed are a subset of the more complex and numerous severe accident phenomena. The Committee concluded that a more comprehensive and ongoing integral assessment program is needed. This program would (1) provide an increased flow of needed information to the code developers regarding code deficiencies, (2) promote the development of user guidelines, and (3) improve the level of knowledge regarding the performance of the code relative to its targeted applications. While encouraging an increasing pace to the integral assessment effort, the Committee emphasizes the importance of maintaining an overall perspective on the role of integral benchmarks vs the qualification of individual models. As previously discussed, a complete assessment of the technical adequacy of an integral code considers both the component parts (bottom-up review) and the adequacy of the integrated coded package (top-down review). The detailed or bottom-up review focuses on the pedigree, applicability, and fidelity of the individual models and correlations. The Committee emphasizes the importance of having technically adequate detailed 
models and correlations which are, in reality, the building blocks of the integral code. Thus, the importance of having technically adequate detailed models cannot be overemphasized as this is a necessary condition for having a technically adequate integral code. References 3-18 and 3-19 contain comparisons to both simple analytical and simple experimental tests. This effort should be continued and expanded where possible.

3.3.3.3. Code-to-Code Comparisons. The most appropriate and direct technique for assessing technical adequacy is by comparison of code-calculated results to analytical solutions or full-scale, prototypical experimental data. In the context of assessing the technical adecuatcy of the total or integrated code, several such assessments have been discussed in the previous section. However, the desired analytical solutions exist for only a relatively few and simple physical processes. Experimental data are also frequently limited, particularly prototypical data for severe accident phenomena. In the absence of the needed benchmarking information, code-to-code comparisons have some value. For severe accident phenomena, their value lies not in the absolute, i.e., what is right and what is wrong, but in focusing attention on what is different. When properly conducted, such efforts can identify and highlight, for example, models that are either incorrect, not applicable for the phenomena being analyzed, or otherwise deficient.

Several code-to-code comparisons have been completed with MELCOR as one of the codes. Results of these comparison studies are abstracted in this report: the Committee did not perform independent code-to-code atssessments. Madni (Refs. 3-20 and 3-3) reports MELCOR and $\mathrm{STCP}$ results for simulations of a long-term station blackout at Peach Bottom. The timing of key events, pressure and temperature response in the reactor vessel and containment, hydrogen production, and the release of source terms to the environment were compared. The timing of key events calculated by the code were, for the most part, in reasonable agreement. However, vessel failure occurred later in MELCOR than in STCP. Debris was immediately ejected into the cavity in the STCP calculation while debris injection was delayed for about $65 \mathrm{~min}$ in the MELCOR calculation. Significant differences in environmental releases were calculated with the two codes. These differences were explored and discussed. Madni noted that MELCOR predicted much lower environmental release fractions of strontium, lanthanum, cesium, and barium, and somewhat higher release fractions of iodine, cesium, and ruthenium than STCP. The higher environmental release fractions of iodine and cesium from MELCOR were attributed to late revaporization from the reactor cooling system after the core debris penetrated the reactor vessel. This phenomena was not modeled in STCP. Madni concluded that the revaporization model in MELCOR "represents an important advance in modeling capability."

Madni also reports the comparison of MELCOR, STCP, and SCDAP results for PBF SFD test 1-1 (Ref, 3-12). Comparisons were performed for bundle water level, power transferred to the bypass coolant, cladding temperatures, fuel temperatures, and hydrogen production. Comparisons to test results were also performed; these comparisons have previously been discussed in Chapter 2. MELCOR and STCP displayed similar calculated trends for the bundle water level. Since flow rates were provided as input to SCDAP, a comparison was not appropriate for bundle water level. All three codes predicted similar power transfers to the bypass coolant. Both MELCOR and STCP displayed similar overpredictions of the integral hydrogen generation. Thus, the focus on hydrogen generation, in this case, was less code-to-code and more on the codeto-data comparison.

Madni continued his code-to-code comparisons by considering MELCOR and SCDAP/RELAP5 calculations of PBF SFD test 1-4 (Ref, 3-13). He concluded that MELCOR Version 1.8 calculations have represented the bundle behavior during the test reasonably well, showing the same trends as SCDAP/RELAP5. Since SCDAP/RELAP5 is the NRC's detailed mechanistic code, such assessments increase confidence in the ability of the integrated analysis code MELCOR to predict in-vessel phenomena. This confidence is strengthened, in this case, because the calculated results of both codes are also compared to experimental data.

Madni's most recent code-to-code comparison effort is reported in Ref. 3-14. In this document, results of MELCOR Version 1.8 DN and SCDAP calculations are compared to each other and to experimental data from FLH'T' test 2. Madni reports that both MEL COR and SCDAP underpredict the sharp temperature rise due to accelerated Zircalloy oxidation and each also predicts 
a delay in the timing of the peak cladding temperalure. Given the avalability of experimental data, the matural focus of Madni's evaluation efforts is on the code-to-data comparisons. Thus, evaluation of code-10-code modeling differences is minimal.

An extensive code-to-code comparison activity involving MELCOR is currently in progress. The industry-sponsored MAAP code is being evaluated by the NRC and comparison of MAAP and MELCOR models as well as full-plant MAAP-calculated results and MELCORcalculated results is a part of this effort. A thorough effort to compare detailed models has been documented in Refs. 3-21 through 3-24. This effout has laid the base for subsecuent evaluations of calculations performed for each code of one BWR accident seyuence (Peach Bottom accelerated station blackout seculence) and one PWR accident sequence (Zion small-break I.OCA sequence). The analysis teams using cach code carried out a coordinated effort to use, where possible, input files for the two codes that were identical. Although some of the comparison calculations have been completed at the time this report is being written, the evaluation of results is still in progress. Even at this preliminary stage, the value of the comparison activity can be seen. Kenton (Ref. 325) reports, for example, that differences in the calculated BWR results focused attention on differing approaches 10 modeling boundary conditions, in-contaimmont heat-transfer processes, and debris heat-transfer processes. Differences in the calculated PWR results focused attention on modeling of the break phenomena and retention of some radionuclides in the primary system.

Although code-to-code comparison cannot and should not displace or replace code benchmarking efforts against test data, the Committee concludes that such comparisons can provide useful supplementary insights. As previously stated, for severe accident phenomena, their value lies not in the absolute, i.e., what is right and what is wrong, but in focusing attention on what is different. When properly conducted, such efforts can identify and highlight, for example, models that are either incorrect, not applicable for the phenomena being analyzed, or otherwise deficient. The Committe recommends that such comparison activities continue in the future.

3.3.3.4. Sensitivity Analyses. The Committee has concluded that review of a comprehensive set of well defined and executed sensitivity analyses is an important and necessary component of the effort to determine technical adequacy. Unfortunately, this comprehensive set of MELCOR sensitivity analyses does not presently exist. Rather, a sparse set of sensitivity studies exist, and several of these only became available during the conduct of the MELCOR Peer Review effort. Two categories of sensitivity analyses are of particular interest and importance in assessing code technical adequacy. Time-step sensitivity stud: as are thought by the Committee to be of the umost importance. Noding sensitivity studies are also believed to be important.

Time-Step Sensilivity Studies. A limited number of time-step sensitivity studies were available to the Committee. Several of these are of recent vintage and highlight a key concern of the Committee. That concern is that the MELCOR code has not been dem.nnstrated to consistently produce a clear trend toward convergence with a monotonically decreasing time step. Several of these points were previously described in Chapter 2 and are repeated here for sonvenience.

SNL notes that several users have observed sensitivities to time-step. increments in some calculations and states "Some of these sensitivities are believed to be unavoitable because of the threshold nature of some models and the complex interplay between competing processes, but others are not yet fully understood" (Ref. 3-23, p. 6-4).

Madni (Ref. 3-3) reports that the selection of the maximum time step, $\Delta t_{\text {max }}$ and its impact on the calculational behavior of MELCOR "is an area of lingering uncertainty in the use of MEL.COR." The impact of varying the maximum time-step specification was explored for a station blackout transient in Peach Bottom using MELCOR Version 1.8BC and $\triangle$ tmax values of $10 \mathrm{~s}$ (reference calculation), $5 \mathrm{~s}$, and $3 \mathrm{~s}$. Madni's results do not display a trend towards convergence. Rather, the largest deviations relative to the reference time step are were associated with $\Delta t_{\max }=5$ $s$ and the deviations were smaller for the 3-s lime step. Thus, $\quad$ trend towards convergence was not observed.

In the most recent sensitivity study reported by Madni (Ref. 3-14), time-step sensitivity was again evaluated. Madni used $\Lambda \mathrm{t}_{\max }=5 .(), 3 .(), 1 .()$ and $0.5 \mathrm{~s}$ beginning at $2(0)$ - $\mathrm{s}$ transient time. Before 2()() s the same $\Lambda t_{m a x}$ was used for each calculation. Madni concluded that the calculations appeared to converge with the selection of smaller $\Delta$ lmax values, "the difference 
between $\Delta t_{\max }=1$ () sec and (0.5 sec being very small." Madni altributed the observed convergence to the fact that the experiment did not involve competing and threshold phenomenat as in a full plant calculation.

Khatib-Rahbar (see presentation 3-8 of Ref, 3-26) discussed time-step sensitivity results and reported "reductions in time step do not necessurily lead to a converged solution" although "bottom line source term results /are / generally less sensitive to time step sizes." The ci, ulations were performed for a BWR with a Mark-I containment using $\Delta t_{\text {max }}$ values of 5,3, and $1 \mathrm{~s}$. The reported times of lower vessel head penetration failures (in MELCOR model ring 1) were calculated as $18,8()(), 10,7()()$, and $19,7()()$ s for $\Delta t_{\max }$ values of 5,3 , and 1 s, respectively. Again, no trend to convergence with reduced maximum time step was observed. Related results were reported in Ref., 3-5.

Robertson (Refs, 3-15 and 3-16) concluded that the MELCOR predictions for BMC-F2 are inaccurate when $\Delta t_{\max }$ is too large. Robertson performed calculations with time steps of $3(0.0$, 3.() , 2.(), 1.0, and $0.5 \mathrm{~s}$. For the calculations performed by Robertson, this deficiency in the predictions was overcome by limiting $\Delta \mathrm{t}_{\max }$ to $2 .($ ) $\mathrm{s}$.

The MELCOR time-step sensitivities observed in the few sensitivity studies performed to date are of major concern to the Committee. This problem should be explored more fully in the near future. Most desirable would be a demonstration thail MELCOR consistently converges once $\Delta t_{\text {max }}$ has been reduced below a certain threshold value. If such a demonstration cannot be provided, guidelines must be developed that will permit the user to obtatin code-calculated results that can be used to satisfy the analysis objectives of the given effort. Such guidelines would, most likely, result from an extensive set of integral benchmarks. There is also the suspicion that timestep sensitivities may indicate other parameter sensitivities and that the time-step sensitivities may mask or overwhelm real physical sensitivities.

Noding Sensitivity Sludies. In concept there are many similarities between the importance of time-step sensitivities and noding sensitivities. However, noding sensitivity studies are more costly in time and effort because the input model and associated input deck must be modified for each noding study. Alteration of $\Delta t_{\max }$ is simple in that only a single input value is changed for each calculation. Many more values must be changed for each noding study. The Committee did not have access to many noding studies. Madni (Ref. 3-14) performed calculations of the FLHT-2 experiment using three different axial nodalizations of the active bundle region (.3), 2(), 10, and 5 axial segments). He reported that there was a noticeable difference between the sensitivity calculations. The axial nodalizations of 3() and 2() segments yielded predictions of key parameters (bundle licuuid level, heat transfer to bypass fluid, hydrogen production, and cladding temperatures) that were acceptably close to the meatsured values. The calculations with 30 and 20 segments were also very similar. Madni reported a noticeable difference between the 20) segment noding and the coarser nodings and selected the 20 segment noding ats the reference noding for his calculations.

A full plant noding sensitivity study was presented to the Committee (Ref. 3-10, presentation 1-19). Two nodalizations were prepared. "The original nodalization and a "simplified" nodalization had about one-half the number of control volumes and flow paths. Ultimately, and after some effort, the understanding gained from working with the simplified model was used to revise the original, more finely noded model. The Commitlee notes, however, that the currently available documentation does not discuss the lessons learned from this activity (Refs. 3-9 and 327 ) and reports only the results of the simplified model. At the current stage of MELCOR development and application, the Committee believes that it is important for all such information be documented and made available to the user community. In this context, that which did not work well is as important as that which did work well, becallse each contributes to an understanding of the limits of applicability of the code.

Because so few noding sensitivity studies have been completed to date, it is difficult for the Committee to make findings in this area. It has heen possible for the Committee to conclude that noding sensitivity studies are an area that should be further explored in order to develop user guidelines.

Qther Sensitivity Studies. Martinez (Ref, 3-11) describes sensitivity studies conducted to assist in the selection of MELCOR impul paramelers. She reports that "several adjustments in 
boundary conditions, heat transfer parameters, and material properties were necessary to improve the temperature comparisons of the MELCOR calculation with bundle temperature data." All such adjustments were limited to the range of uncertanties for the affected paramelers. 'The Commiltee recognizes the existence of uncertainties related to defintion of the values of input parameters required by MELCOR. The Committee also recognizes that input parameters can be adjusted to improve the comparison to measured data and, in doing so, mask real problems by compensating for their impact through input parameter adjustments. 'The Committee recommends that user guidelines be prepared that require full disclosure of any such input parameter adjustments and a discussion of their impact in released documentation.

3.3.3.5. Committee Observations. MELCOR Objective 2 states that "MEL.COR should provide reasonable predictions of the progression and consequences of severe accidents." The Committee collected and reviewed the available information regarding (1) numerics, (2) comparison to integral benchmarks, (3) code-to-code comparisons, and (4) sensitivity analyses. Findings relative to these subjects have been reported in previous sections of this report. If a thorough and complete set of studies had been completed, documented, and available, the preparation of definitive findings regarding the accomplishment of MELCOR OLjective 2 would be relatively straightorward. 'This statement assumes the existence of the information needed to complete a thorough bottom-up review of the individual models and correlations which, taken together, comprise the integral or total code. However, such is not the case and the information available to the Committee related to the behavior of the integrated code was limited. Thus, af fifth source of information is provided, the observations of the collective MELCOR Peer Review Committee and its individual members. Although such opinions will never adeyuately substitute for "hard" information in the four categories listed above, it is hoped that they will be of some value in the environment of limited "hard" information.

Review of a Full-Scope MELCOR BWR Calculation Results of a MELCOR calculation of a postulated BWR short-term station blackout accident sejuence were provided to the Committee on two occasions. The calculations represented the physical configuration of the LaSalle County Station, A BWR/5 with a Mark-II containment structure, and were full scope in their evaluation of severe accident behavior. That is, the calculations addressed in-and ex-vessel aspects of core melt progression, the accompanying containment thermal-hydraulic response, and altendant fission product release and transport to the environment.

The first calculation was performed with MELCOR Version 1.8.(). It was provided to the Committee very early in the review process as an introduction to the corde's capabilities (Ref. 310). Overall, the results of this calculation appeared reasonable in that the calculated seyuence of events and values of many important parameters, such as total hydrogen production, appear to be consistent with those made in earlier studies with other computational tools. However, several deficiencies in selected portions of the calculation were observed by the Committee, many of which had already been identified by SNL staff. Some of these deficiencies have subseguently been addressed via modifications to physical process models and/or their reduction to coding, and are incorporated in code changes used to generate MELCOR Version 1.8.1, July 1991.

The second calculation addressed the same BWR accident scenario and was performed at a later time with a preliminary version of 1.8.1. The combination of these two calculations illustrate the strengths and remaining weaknesses of the current code models and underscore the developmental status of MELCOR. Noteworthy findings or observations from the Committee's review of these calculations are outlined below.

1. Substantial differences in important calculated results from the MELCOR Version 1.8.() and preliminary 1.8.1 calculations were observed. Some of these differences indicate improvements in code models or their implementation. For example, large energy errors in the COR package and mass balance deficiencies in the RN package observed in the MELCOR Version 1.8.() calculation appear to have been eliminated or reduced in the subsequent MELCOR Version 1.8.1 calculation. Other differences, however, clearly illustrate the lack of maturity (or continuing development) of some MELCOR models. For example, in-vessel hydrogen production differed by $17 \%$ in the two calculations, 
the to containment failure chunged from $48,86,3 \mathrm{~s}$ in the first calculation $1024,6,31 \mathrm{~s}$ in the second calculation, and radionuclide release to the enviromment decreased by a fiactor of 2 to 10, depending on species from the first to the second calculation.

2. In both calculations, the reactor vessel failed via a penetration fiallure approximately 2 min after molten debris began to relocate to the lower plenum. "This occurred in spite of a relatively small mass of molten $\mathrm{UO}_{2}$ (approximately $140 \mathrm{~kg}$ ) entering approximately $75 \times 10)^{3} \mathrm{~kg}$ of water in the lower plenum and reflects the lack of an in-vessel molten debris-coolant interaction model.

3. Some details of the calculated in-vessel core melt progression (in particular, results related to material relocation) are surprising and warran further inspection. For example, when the first ring of the lower core support plate failed, $\mathrm{UO}_{2}$ mass in the cell containing the core support plate decreased to zero, reflecting its relocintion to the lower plenum. $\mathrm{At}$ the same time, however, the Zircalloy mass in the same cell sharply increased and remained constant for nearly $4(0)()$ s before decreasing to zero. Over the same period, a comparison of temperatures of the debris field in the COR material cells immediately above the lower core support plate and the temperature of debris arriving in the lower head suggests that the latter originated in COR cells substantially higher than the one containing the support plate.

4. Large temporal variations in the airborne mass and size distribution of aerosols throughout the problem are calculated and appear to have no physical explanation. In particular, the aerosol mass in virtually all "sections" of the size distribution is observed to change in a near-oscillatory fashion during a period of the accident when relatively little else is occurring, i.e., the period between drywell pedestal floor failure and containment fuilure.

5. Finally, SNL's approach to this BWR calculation, as well as the experiences of at least two Committee members with the application of MELCOR 1.8.(), suggest substantial and, perhaps, an excessive need for the code user to "adjust" input parameters to yield a plausible sequence of in-vessel events. Melting, relocation, and refreezing of BWR fuel canister and control blade materials are observed to be strongly influenced by, among other things, the user's selection of criteria for cladding oxide shell failure, debris and lower core support plate porosity, and selected melt-structure heatt-transfer coefficients. In the absence of clear guidance for, at a minimum, a reasonable range of appropriate values for such parameters, an inexperienced user is placed in the position of arbitrarily choosing values that generate his/her view of a "correct" answer.

Review of a Full-Scope MELCOR PWR Calculation. At the request of the Committee, SNL performed a calculation of a Surry station blackout (SBO) accident with MELCOR, A similar calculation, a Zion small LOCA transient, is currently being conducted all BNL as part of a MAAP/MELCOR comparison exercise (Ref. 3-2.5). These are the first fully integrated PWR severe accident calculations that have been performed with the code. As such, the Committee was very interested in reviewing the Surry results, since they presumably could provide an overall or top-down impression of the ability of the code to model PWR accident seyuences.

In general, the Committee was favorably impressed with the results of the Surry calculation. Based on observations of SBO predictions made with other codes, the results appear "reasonable," with some exceptions that will be discussed below. We were able to review both plotted output and a computer-generated "movie" of the accident progression. SNL has clearly devoted considerable effort to the development of techniques for displaying MELCOR results; this is of great value when trying to digest the nearly overwhelming amount of informattion generated in an integral severe accident calculation.

As in several of the other calculations presented to the Committee, SNL, found it expedient to first simplify the inpul deck, which in this case was based on a model developed for RELAP/SCDAP at INEL. Both the number of control volumes and the number of healt sinks were reduced. This appears to reflect both a desire to simplify the process of model shakedown and to speed up execution. After this was done, there were evidently no severe problems in getling the calculation to run. Run times were aceeptable at $6(2 \%$ of real time on a $\vee A X 87())$. Of course, 
SNI. was responsible for this calculation, and they related to the Commillece sente "lore" that they used when constructing the input deck to obtain aceeplable results. For example, the opening height used on the junction that represents the pressuriger PORV was made rensomuble lange (().5 m was used in the Surry model) to obuln necephable run times. Nol surprisingly, informal feedback from the MAAP/MLiL COR comparison project indicales that less experienced asers may have more dillicully (Reli, .3-2.5).

We did not perform an exhanstive review of the Surry results, primarily because the Commilted did not allocale suffictent resoureses and time to this effort. Based on our brief review, some of the results were staprising or at least noteworthy, and hese are listed below.

1. Alter the stenm generators dried out, the pressurizer level rose and remained within $2 \mathrm{~m}$ of the top of the unit until rencelor vessed failure. RELAP results (Ref, 3-28) and hand calculations indicale that entrainment of waler when the power-operated rellef valves (PORVs) lift should lower the water level further than this after the surge line uncovers. Counterentent flow of waler and steam in the surge line, which is modeled cerlodely by CVH by using a flooding correlation, should also reduce water level in the long term. Neither effect is very significant in the MELCOR calculation.

2. Primary system gats cemperatures were yuite low, especially in line coolant lexps. Part of this is explained by the face that core/upper plenum and upper plenum/steam generator matural circulation before reactor vessel failure are not currently represented in MELCOR. In addition, it appears that the loop seals in the reactor coolant pump suction piping did not clearr, even long after vessel failure, so that unidirectional circulation around the entire primary system was precluded. While not unreasonable, this is a striking result.

3. In-vessel hydrogen production was yuite low, only 19() $\mathrm{kg}$. 'This may be due, in part, to steam starvation, callsed by the failure to model matural circulation between the core and the upper plemum.

4. The reactor vessed failed very soon after debris relocated to the lower plenum. This reflects a modeling change in Version 1.8.1. Still, it should be recognized that the code does not model the breakup of molen debris as it enters the lower plenum, so there is appatrently no way 10 mechanistically avoid prompt RPV failure in this sequence.

5. One of the advantages of a unified modeling approach is evident in the steam generator results. After the steam generators dried out, a noticeable natural convection flow was calculated between the downeomer and tube bundle. Given the heat load from fission products deposited on the primary side of the tubes, this is to be expected, but other severe accident coles that use a more coarse modeling could not explicilly predict this effect. Such a capability would be useful for making estimates of peak tube temperuture in studies evaluating inducted steam generator tube ruptures (Ref. 3-29). This illustrates the potential advantages offered by a flexible, fully integrated code such as MELCOR.

6. It is clear that the containment results would be greatly affected if debris dispersal from the reactor cavity, which cannot be modeled currently, was taken into account. The same is true for induced hot-leg or surge-line rupture.

7. Cavity and upper contaimment waller mass fluctuated in an erratic fashion after vessel failure. 'The reason for this was not known.

In summary, the Committee was favorably impressed by the overall performance of MEICCOR, especially considering the fact that this is one of the very first integral PWR calculations that has been performed with the code. Still, some of the results are not understood and others illustrate the importance of adding some of the key models that are currently missing. Having found even this brief review to be quite valuable, the Committee recommends that additional integral assessments of MLILCOR be performed, including comparisons to other codes. 


\subsection{Objective 3}

\subsubsection{Statement}

MELCOR should permit estimates of the uncertainties associated with its predictions of severe core damage accidents to be made without requiring modifications to the code.

\subsubsection{Supporting Definitions}

The Committee interpreted this requirement as the ability to perform sensitivity studies without requiring modifications to the code.

\subsubsection{Findings for Objective 3}

The Committee has found that Objective 3 has been satisfled. SNL has implemented a flexible sensitivity analysis capability. Other parameters in correlations, which are hardwired constants in many codes, are implemented in MELCOR as variables called sensitivity coefficients, which can be changed by the user through imput (Ref. 3-23). Although the calpability to perform such sensitivity and uncertainty studes exists, it is less clear that this capability has been used as envisioned by the objective statement. As discussed above, few sensitivity analyses, particularly those envisioned by the objective statement, have been conducted to date. There is also a suggestion that the assessment of MELCOR uncertainties has not been done (Ref. 3-3()). The anthors comment that "many models considered in practice are far more complex than the three models used in this comparison. For example, the MELCOR computer program indicated in the introduction is such a model."

\subsection{Objective 4}

\subsubsection{Statement}

Models incorporated in MELCOR should be in adeyuate detail to address important phenomenology but permit a practical rumning time for accident sequence analysis.

\subsubsection{Supporting Definitions}

The commitlee chose to interpret this statement in the context of total resource commitment. More specifically, the committee feels that the resource commitment is the summation of four components: (1) the cost to develop the plant model/input file, (2) the cost associated with getting to the final calculation (e,g., how difficult is it to obtain the correct results and produce the calculation that is relatined as the answer), (3) the cost per final calculation, and (4) the cost 10 analyze and interpret the results. The commillee yuestioned if the "practical running lime" specification was developed a number of years ago when computational costs were higher. SNL noted that MELCOR was operational on many systems from a supercomputer (Cray), to midsize (VAX), to desk-top equipment (IBM-RISC and Sun), Given that computation times are rapidly decreasing, the costs associaled with items 1, 2, and 4, above, are now more important than ilem ?.

The Committe also considered several alternative and more yuantitative specifications that are specific to the "practical running time" question. For example, it was proposed that for a specified noding (sily 1.5-20 control volumes) and a given computer (Cray), the run time be less than $2 \mathrm{~h}$. Another proposial was that the code run faster than real time, which would also require a noding and computer specification. The Committee chose to not accept these alternate and more yuantitative specifications. 


\subsubsection{Findings for Objective 4}

The Committee concluded that the code currently satisfies the practical running lime objective as modified in Item 2, above. The Committee notes, however, that this finding is based on the assumption that the code is applied by the targeted user community; the NRC has identified this user community as the NRC itself and the national laboratories. Finally, it should be noted, however, that some of the important phenomenology are not currently modeled in MELCOR. Missing models identified by the Committee are discussed in Chapter 4.

\subsection{Objective 5}

\subsubsection{Statement}

MELCOR should be appia:-ahle for severe accident studies and related plant applications for both PWRs and BWRs.

\subsubsection{Supporting Definitions}

This objective applies to the cotal or integral performance of the code. The applicability of the individual models and correlations (bottom-up) review of the code is covered in the discussion of Objective 1.

\subsubsection{Findings for Objective 5}

Coverage of the important features related to Objective 5 are found elsewhere in the Committee's report. As noted above, Committee findings regarding Objective 2 maturally fall in the category of top-down or integrated code capabilities. In addition, Objective 5, as stated, is closely related to the subject of targeted applications discussed in Chapter 4. The reader is referred to these discussions for findings regarding Objective 5 .

\subsection{Objective 6}

\subsubsection{Statement}

MELCOR should be portable, maintainable, and have a structure that facilitates incorporation of new or alternative phenomenological models.

\subsubsection{Supporting Definitions}

This objective has no supporting definitions.

\subsubsection{Findings for Objective 6} follows.

The Committee finds that Objective 6 has been satisfied. The basis for this finding

3.7.3.1. Portability. To enhance portability, ANSI Standard FORTRAN 77 was followed (Ref. 3-23) during MELCOR programming activities. Nonstandard or processordependent (i.e., non-ANSI) coding has been used only where unavoidable (e.g., calls to system routines to obtain time/date and central processing unit information, for which no standard exists). Any nonstandard coding has also been identified by appropriate comments and bracketed by appropriate conditional symbols required by UPDA'TE. 
3.7.3.2. Maintainable. In a sense, Committee conclusions related to maintainability of MELCOR must be inferred. The Committee notes that SNL has implemented a system to control maintenance of the code. MELCOR maintenance procedures are described in Ref. 3-23. Elements of the maintenance effort include basing maintenance on UPDATE, a system utility on Cray and CDC; HISTORIAN, a commercially available emulator; and Code Maintenance Package or CMP, which is a SNL-developed package of FORTRAN utilities. All additions, corrections, and improvements to MELCOR are tracked using Defect Investigation Reruests (DIRs). The steps in the DIR procedure are (1) request, (2) diagnosis, (3) plan, (4) changes/testing, and (5) implementation. SNL is the designated custodian of all official MELCOR code versions. The Committee concludied that the combination of code characteristics and SNL procedures are sufficient to demonstrate successful completion of this subobjective.

3.7.3.3. Structure that Facilitates Incorporation of New or Alternative Phenomenological Medels. The Committee was provided sufficient evidence to conclude that MELCOR does have a structure that facilitates incorporation of new or alternative phenomenological models. As described in Ref. 3-23, a modular, highly structured, and userfriendly architecture was required to facilitate both the initial development of the code and the later modification and/or addition of phenomenological models. A well-defined, top-down code structure was developed with careful attention given to the structure of the MELCOR data base and the data-base manager routines. Basic structural guidelines and coding conventions were established to promote modularity, readability, and ease of modification.

Several examples that demonstrate satisfaction of this subobjective exist. The MELCOR code was recently used in support of the N Reactor PRA (Refs. 3-31 and 3-32). The authors note that although MELCOR was able to model the logic, the differences between the N Reactor and a commercial LWR core required several modification efforts. The first area of effort was adaptation of support and noncore physics modules. The authors note that the restrictions associated with the MELCOR coding conventions would not allow for all the new $N$ Reactor required models to be included in the revised core module (package). Instead, several MELCOR support modules were modified to model phenomena only relevant to the $\mathrm{N}$ Reactor core module. The modified support modules included the executive, radionuclide, material properties, and decay heat modules. The authors concluded. "The extreme differences between $N$ Reactor and typical LWRs made the modification of the existing MELCOR Core package impractical. Instead, a new module was written to describe $N$ Reactor core phenomena. MELCOR's highly modular structure made possible the rapid integration of the new core model with the existing hydraulic and radionuclide models." The successful completion of this effor supports the conclusion that this MELCOR subobjective has been satisfied.

A second example of an extensive MELCOR modification effort exists. MELCOR has been modified for the analysis of heavy water moderated, U-Al fuel reactors (Ref. 3-33). The authors note that MELCOR is modular in structure; that is, the FORTRAN representation of process models is separated into several distinct packages, each of which addre's a 'well-defined group of closely related phenomena. They further state, "This architecture facilitates the incorporation of additional or alternative phenomenological models." The modifications to MELCOR for the heavy' water reactor application were extensive. A number of phenomenological packages were modified. BUR was modified by including $\mathrm{D}_{2}$ as a combustible gas. CAV was modified to account for heavy water reactor materials and chemistry. As with the $N$ Reactor effort, a new COR package was written. A default decay heat curve applicable to the heavy water reactor was added to DCH. Parametric models representing the energetics and fission product release from debris-coolant interactions of various magnitudes were added to FDI. Enhancements to the radiation heat-transfer models were made to $\mathrm{HS}$. Thermophysical properties of Al, U-Al, Cd, Li$A l$, and $D_{2}$ (gas) were added $10 \mathrm{MP}$. $\mathrm{D}_{2}$ gas was added to the noncondensable gas flow field in NCG. A new fission release model for molten pools of $U$-Al, reaction chemistry in VANESA, and enhancements to fission product deposition models in RN were prepared. Finally, augmented filter models were provided. The successful completion of this effort supports the conclusion that this MELCOR subobiective has been satisfied. 


\section{REFERENCES}

3-1. F. Eltawila, Accident Evaluation Branch, Division of Systems Research, Office of Nuclear Regulatory Research letter about MELCOR objectives and targeted applications to B. E. Boyack, Los Alamos National Laboratory (November 2, 1996).

3-2. G.G. Weigand, "Thermal-Hydraulic Process Modeling in Risk Analysis: An Assessment of the Relevant Systems, Structures, and Phenomena," Sandia National Laboratories report SAND84-1219 (NUREG/CR-3986) (August 1984).

3-3. I. K. Madni, "MELCOR 'Lessons Learned': A User Perspective," Brookhaven National Laboratory draft letter report to R. Foulds, United States Nuclear Regulatory Commission (January 9, 1991).

3-4. B. E. Boyack and J. Richardson "Final Minutes of the Third MELCOR Peer Review Meeting," Los Alamos National Laboratory Group N-12 letter N-12-91-171 (March 20, $1991)$.

3-5. S. L. Chan, H. P. Isaak, U. Schmocker, and M. Khatib-Rahbar, "Characterization of Severe Accidents Using MELCOR: A Perspective," Swiss Federal Nuclear Safety Inspectorate, Presentation at the Cooperative Severe Accident Research Program SemiAnnual Review Meeting, Bethesda, Maryland (May 6-10, 1991).

3-6. R.M. Summers, R.K. Cole, Jr., E.A. Boucheron, M.K. Carmel, S.E. Dingman, and J.E. Kelly, "MELCOR 1.8.(): A Computer Code for Severe Nuclear Reactor Accident Source Term and Risk Assessment Analyses," Sandia National Laboratories report SAND9()-(0364 (NUREG/CR-5531) (January 1991).

3-7. C. R. Hyman, "Current Limitations in the Application of "CONTAIN/ CORCON to BWR Mark I Severe Accident Analyses," Oak Ridge National Laboratory document (January 27, 1989).

3-8. E. L. Fuller and J. A. Maly, "Progress in MAAP Development and Use as Illustrated by Station Blackout Accident Studies," Electric Power Research Institute, Presentation at the Cooperative Severe Accident Research Program Semi-Annual Review Meeting, Bethesda, Maryland (May 6-10, 1991).

3-9. E.A. Boucheron and J.E. Kelly, "MELCOR Analysis of the Three Mile Island Unit 2 Accident," Nuclear Technology 87 (December 1989) p. 1050.

3-10. B. E. Boyack and J. Richardson, "Final Minutes of the First MELCOR Peer Review Meeting," Los Alamos National Laboratory Group N-12 letter N-12-90)-518 (September $5,199()$.

3-11. G. M. Martinez, "MELCOR Calculations of ISP28 SFD PHEBUS Test: B9+," Sandia National Laboratories letter report transmitted to B. Adroguer (December 14, 1990).

3-12. I.R. Madni, 'MELCOR Simulation of the PBF Severe Fuel Damage Test 1-1," AICHE Symposium series, Proceedings of the 26th National Heat Transfer Conference, Philadelphia, Pennsylvania (1989).

3-13. I. K. Madni, "MELCOR Modeling of the PBF Severe Fuel Damage Test 1-4," Brookhaven National Laboratory document BNL-NUREG-445()3 (1990). 
3-14. I. K. Madni and X. D. Guo, "MELCOR Simulation of the Full-Length HighTemperature 2 Experiment," Brookhaven National Laboratory Safety Integration Group document (1991).

3-15. M.1. Robertson, "MELCOR-A Modelling Overview and Calculations for Phase 1 of the BMC-F2 Experiment," United Kingdom Atomic Energy Authority, Safety and Reliability Directorate report PWR/SAWG/P(90)578, PWR/FPERG/P(90)108 (May 1990).

3-16. M. I. Robertson, "Validation of Control Volume Thermal-Hydraulic Modeling," CSNI/CEC Workshop on Aerosol Behaviour in the Containment, Fontenay-aux-Roses, France (November 1990).

3-17. G. M. Martinez, "MELCOR Post-Test Calculations of the HOR Experiment," Sandia National Laboratories letter report to R. B. Foulds (September 29, 1989).

3-18. C.D. Leigh, "MELCOR Validation and Verification; 1986 Papers," Sandia National Laboratories report SAND86-2689 (NUREG/CR-4830) (March 1987).

3-19. C.D. Leigh, R.K. Byers, and C. J. Shaffer, "MELCOR Validation Results," Proceedings of the 14 th Water Reactor Safety Research Information Meeting," Gaithersburg, Maryland, Sandia National Laboratories paper SAND86-2128C (NUREG/CP-()()82) (October 27-31, 1986).

3-20. I. K. Madni, "MELCOR Simulation of Long-Term Station Blackout at Peach Bottom," Proceedings of the 18th Water Reactor Safety Information Meeting, Brookhaven National Laboratory document NUREG/CP-()113 (October 1990).

3-21. J. W. Yang and D. Mirkovic, "MAAP 3.()B Review/PWR Review, Part 1: RCS Thermal Hydraulics and Heat Transfer, Application for Success Criteria," Safety and Risk Evaluation Division, Department of Nuclear Energy, Brookhaven National Laboratory draft document (May 1990).

3-22. J. W. Yang, "MAAP 3.0B Review/PWR Review, Part 2: In-Vessel Melt Progression," Safety and Risk Evaluation Division, Department of Nuclear Energy, Brookhaven National Laboratory draft document (August 1990).

3-23. J. U. Valente, "MAAP 3.0B/BWR Review, Technical Evaluation Report 3: Severe Accident Containment Modeling," Safety and Risk Evaluation Division, Department of Nuclear Energy, Brookhaven National Laboratory unnumbered document (October 199()$)$.

3-24. J. W. Yang, "MAAP 3.()B Review/PWR Review, Part 4: Fission Product Release and Transport," Safety and Risk Evaluation Division, Department of Nuclear Energy, Brookhaven National Laboratory draft document (December 199()).

3-25. M. Kenton, "Transmittal of Viewgraphs on MAAP/MELCOR Comparison Calculations," Gabor, Kenton and Associates. Inc. letter to B. Boyack (April 9, 1991).

3-26. B. L. Boyack and J. Richardson, "Final Minutes of the Fourth MELCOR Peer Review Meeting," Los Alamos National Laboratory Group N-12 letter N-12-91-382 (June 4, $1991)$.

3-27. E.A. Boucheron and J.E. Kelly, "MELCOR Analysis of the TMI-2 Accident," Transactions of the American Nuclear Society and the European Nuclear Society 1988 International Conference on Nuclear Fission: Fifty Years of Progress in Energy Security 
and Topical Meeting on TMI-2 Accident: Materials Behavior and Plant Recovery Technology 57, Washington, DC, Sandia National Laboratories paper SAND88-1258C (October 30-November 4, 1988) p. 435-436.

3-28. P. D. Bayless and R. Chambers, "Analysis of a Station Blackout Transient at the Seabrook Nuclear Power Plant," EG\&G Idaho, Inc. document EGG-NTP-67(0) (September 1984).

3-29. K. N. Fleming, et. al., Risk Management Actions to Assure Containment Effectiveness at Seabrook Station," Pickard, Lowe, and Garrick, Inc, document PLG-()55() (July 1987).

3-30. R. L. Iman and J. C. Helton, "An Investigation of Uncertainty and Sensitivity Analysis Techniques for Computer Models," Risk Analysis 8, No. 1 (1988) p. 71-90.

3-31. L.A. Miller, G.D. Wyss, D.M. Kunsman, S.E. Dingman, E.A. Boucheron, M.K. Carmel, and C.J. Shaffer, "N Reactor Probabilistic Risk Assessment Supporting Calculations," Volume 1: Main Report, Sandia National Laboratories report SAND892101 (June 1990).

3-32. G.D. Wyss, R.M. Summers, and L.A. Miller, "MELCOR Adaptation and Validation for Modeling of $\mathrm{N}$ Reactor Core Phenomena," to be published in Proceedings of the International Topical Meeting on the Safety, Status and Future of Non-Commercial Reactors and Irradiation Facilities, Boise, Idaho, Sandia National Laboratories paper SAND90-0115C (September 30-October 4, 1990).

3-33. M. T. Leonard, K. A. Williams, and J. P. Church, "Modifications to MELCOR for the Analysis of Heavy-Water Moderated, U-Al Fuel Reactors," Proceedings of the International Topical Meeting on the Safety, Status and Future of Non-Commercial Reactors and Irradiation Facilities, Boise, Idaho (September 31-October 4, 199()), American Nuclear Society accession number ISBN ()-89448-155-X. 


\section{FINDINGS RELATIVE TO TARGETED APPLICATIONS}

\subsection{Identification of Targeted Applications}

As identified by NRC (Ref. 4-1), the key applications of MELCOR are as follows:

1. PRAs and reviews of PRAs,

2. audit reviews of IPEs,

3. accident sequence studies to develop insights into phenomena and hardware performance, and

4. AM studies.

For our purpose here, the first two of these are equivalent, and we will direct much of our attention to MELCOR's capabilities in these areas. Accident sequence studies directed toward investigating phenomena are not substantially different than PRA applications. Hardware performance studies are an important subset of AM studies. With these considerations in mind, the Committee chose to focus on PRA and AM, reasoning that explicit treatment of the other applications would not substantially change any conclusions.

The entire area of accident management is rather ill-defined at present, and this presented problems for making findings in this area. Some people in the severe accident research community, including some members of the Committee, view accident management studies as necessarily involving mechanistic calculations of difficult phenomena such as would be encountered by reflooding a damaged core. In Ref. 4-2, however, a senior NRC official stated that MELCOR's usage for accident management would not include such difficult-to-model areas. Instead, the code would be used to provide general insights on the effects of different strategies for coping with a severe accident. It was further stated that a parametric capability would suffice for phenomena that are poorly understood.

Written direction to the Committee (Ref. 4-1) explicitly states that MELCOR is to be used to evaluate the effectiveness of various recovery strategies and the consequences of alternative mitigation strategies. As discussed above, this was read by some Committee members to require that the code should be able to, for example, estimate the amount of hydrogen that would be produced upon reflooding a damaged core or be able to evaluate whether containment flooding can prevent liner melt-through in a Mark-I contaimment. To resolve this matter, the Committee ultimately chose to briefly address such capabilities, reasoning that MELCOR might well be applied in such an arena whether this was officially endorsed or not. The major focus of the Committee's deliberations was to PRA, however.

\subsection{Screening for Missing Phenomenological Models}

The AM area notwithstanding, the most important set of MELCOR applications today and in the near future is for PRA. MELCOR's ability to support either the performance of PRAs or outside reviews of completed PRAs is adversely impacted by inadequate modeling of important phenomena, or, perhaps even worse, areas where models do not currently exist at all. The former deficiencies were identified by separately reviewing the separate packages from the bottom up; the individual package reviews that resulted were discussed earlier.

Our emphasis in this section is on listing models that are not currently in the code but are important for the targeted applications. To provide the sharpest possible focus when screening for missing models, the Committee required that phenomena associated with a nonexistent model had to be important when assessed against specific figures of merit. In the case of PRA applications, the lack of the model had to significantly compromise either calculations of the time of contaimment failure or the environmental release of fission products. Together, these two yuantities determine the consequences of a given accident sequence.

The Committee believes that all the models necessary for PRA are also necessary for AM studies. The converse is not always true, especially if one considers mechanistic calculations of accident recovery, and the Committee separately identified missing models that only impact the use 
of the code for such studies. As discussed above, it is not clear at this juncture whether this is, in fact, a likely role for MELCOR. These missing models are listed below after the PRA issues. The figures of merit used to screen for AM model importance were that the model had to significantly impact the calculation of the key times of the accident sequence, e.g., the time of core damage, core relocation into the lower plenum or vessel breach, or that the missing model was important in calculating the effects on the accident progression of various recovery strategies.

An additional group of missing models, which would be desirable for PRA and important for even parametric AM applications, was also identified. Finally, a number of other models missing from MELCOR, of less importance than those listed here, were also identified. These are noted in Appendix L.

In this context, it should be noted that in a few cases the models are "less important" only in that it was felt that an integrated code such as MELCOR was not the place for the model. For example, hydrogen detonation is clearly a very important issue. Nevertheless, given the lumpedparameter structure of the code, the Committee judged it more appropriate that more detailed models be exercised outside of the MELCOR code structure and that no detonation model be incorporated into the code. Where appropriate, the results of a hypothesized detonation, e.g., prompt containment failure, can be input to the code to estimate the effects of such an occurrence; this is typical PRA practice. MELCOR predictions of hydrogen distribution could be used to provide boundary conditions for such outside analyses. Thus, the list of models in Appendix $\mathrm{L}$ is not meant to imply a serious lack of completeness, but rather is intended to put MELCOR results in perspective by indicating some phenomena that may in certain circumstances be important, but which are not explicitly modeled.

All told, the Committee identified seven models that were considered important for performing either PRA or AM studies that were missing from the code. These will be discussed separately below. In each case, the types of accident sequences most affected by the lack of the model are identified. In a few cases, efforts are already funded at SNL to develop the missing models; this is noted in the discussion. The adequacy of models currently under development was not assessed. The ordering of the models within each of the categories is arbitrary and should not be read to imply a prioritization.

As stated above, when the screening process was over, the Committee found that missing models could be naturally divided into the following three categories.

1. There are missing models that are necessary for PRA applications.

2. There are missing models that, while less important, would significantly improve the code's capabilities for PRA and for parametric accident management studies. These models, in the Committee's judgment, could be practically developed in a relatively short time within the limitations of the existing severe accident data base.

3. There is a missing model that would be needed if MELCOR was ever to perform mechanistic AM calculations of the effects of various recovery strategies. In some cases, it was felt that the development of such models was problematical given the existing level of understanding of severe accident phenomena.

\subsection{Missing Models Necessary for PRA and AM Applications}

The following phenomena were judged to be necessary for both PRA and AM studies and are not currently modeled in MELCOR.

1. PWR primary system natural circulation: Natural circulation is represented in MELCOR for cases in which flow only passes through a given junction in one direction at a time (unidirectional flow). Based on scale model simulations of high-pressure PWR accident sequences and analytical results from other codf:s, situations exist in which countercurrent flows are important. For example, in PWR station blackout transients, steam and hydrogen can flow toward the steam generators in the top of the hot leg and return from the steam generators 
in the bottom. Similarly, rather complex flow patterns have been seen to develop in PWR steam generators in such sequences, i.e, there is flow away from the inlet plenum in a fraction of the tubes and back to the inlet plenum in the remainder of the tubes. Finally, core to upper plenum natural circulation flows can develop in which gas passes down the outer fuel channels and up the inner channels. These flow patterns cannot be credibly simulated within the current MELCOR CVH and control volume temperature distribution (DTDZ) schemes in our judgment. Failure to model such flows seriously impacts the calculation of structure temperatures, hydrogen production, and fission product retention in high-pressure PWR sequences. SNL has stated that a modeling effort to address the core-upper plenum portion of the overall problem is funded and will begin in fiscal year (FY) 1991 and has indicated their belief that the existing $\mathrm{CVH}$ model can deal with the hot leg and steam generator flow patterns. No effort has been identified to either demonstrate an existing capability to model the hot leg and steam generator flows or to develop additional models.

2. High-pressure melt ejection (HPME) and direct containment heating: If the reactor pressure vessel (RPV) lower head fails at high pressure, there is a wealth of data that implies that the debris will not normally remain within the volume underneath the RPV (i.e., pedestal or reactor ('avity). The fact that the debris is removed from this volume by itself greatly alters the subsequent accident progression by changing the boundary conditions for core-concrete and core-water pool interactions. More controversial is whe her the debris will be transported into the bulk of containment as a relatively coherent mass or whether it will be dispersed in the gas stream as fine droplets. The latter, of course, would give rise to the direct containment heating phenomenon, which potentially can impose a large heat and hydrogen load on the containment. MELCOR cannot currently model either process, although a modeling effort is stated to be funded and will begin in FY 1991. This omission compromises the code's use for some important PRA sequences as well as for audit calculations of the results of other codes.

3. Ice condenser: No model currently exists for PWR ice condensers. This obviously prevents the application of MELCOR to this important class of plants. An effort to rectify this omission is to begin in FY 1991.

4. Nonexplosive interactions between debris and water: MELCOR's treatment of the interactions between debris and water is quite limited and internally inconsistent. For example, quenching of particulate debris beds is calculated within the RPV, but MELCOR does not allow this to occur in containment volumes. Contrariwise, fragmentation of molten debris passing through water is only calculated in the containment, not in the primary system. The potential for breakup of a debris crust, which forms on molten debris pools covered by water in the containment, is not treated either. These are areas of great importance and uncertainty, and the lack of even a parametric capability to consistently model such phenomena signilicantly reduces MELCOR's capabilities in both PRA and AM for all accident sequences. The inability to simulate debris-yuenching can in some sequences produce an overstatement in the time of containment failure and can actually predict smaller fission product releases than if quenching was assumed. When a model for these processes is added to the code, it will also be necessary to model the remelting of debris beds if they subseguently dry out.

5. Fission product vapor scrubbing: While some yuestions were raised in the botom-up review, MEI.COR has a relatively sophisticated model for scrubbing aerosols in water pool. In some sequences, however, gas temperatures in the primary system maly be sufficiently high that volatile fission product vapors, rather than aterosols, would be discharged into pools. MELCOR does not 
represent the removal of these vapors as they condense to aerosols and attempt to pass through the pool. Examples of sequences where this process would be important would include low pressure BWR sequences with discharge of vapor through the safety relief valve (SRV) lines to the suppression pool or lowpressure PWR containment bypass sequences with discharge to a water pool in the auxiliary building. In such sequences, this phenomenon could be of umost importance for the calculation of the source term, especially when other fission product removal mechanisms are weak.

6. Models for some RCS deposition processes: MELCOR's fission product deposition models are adapted from the MAEROS containment model. As such, certain processes which are not generally important in the containment have been neglected. These include impaction and turbulent deposition of aerosols. Experimental data on containment bypass sequences performed for EPRI (Ref. 4-3), as well as calculations using more comprehensive aerosol deposition models, indicate that the neglect of these processes may result in a significant understatement of the retention of aerosols in the primury system, especially for low-pressure sequences in which gas velocities are high.

7. Fission product reactions with surfaces: Chemical reactions between setlled aerosols and vapors and heat sinks in the primary system can greatly affect deposition (chemisorption) and revaporization rates. The MELCOR RN package contains a framework by which reactions between vapors and surfaces can be represented, but it is up to the user to perform the actual modeling. The lack of explicit modeling applies to all accident sequences and is particularly serious for cesium hydroxide and tellurium compounds.

\subsection{Missing Models Desirable for PRA and Parametric AM Applications}

The models in this group are considered less critical than those listed above but are still highly desirable for improving the accuracy of source term calculations in PRAs. For AM studies, including those which are parametric in nature, the models are considered even more important. In all cases, the Committee judged that the existing knowledge base is sufficient for developing simple yet credible models for all of these phenomena.

1. Fission product pool chemistry: The chief concern is that the release of iodine to the environment may be understated by MELCOR because the code neglects processes that can occur in water pools to transform cesium iodide into more volatile forms of iodine. This affects all accident sequences, especially lowpressure BWR sequences with substantial deposition in the suppression pool, but is expected to be a rather slow process.

2. Core, pressurizer, and once-through steam generator sprays: The MELCOR containment spray model cannot be used in an all-steam environment. Thus, certain PRA sequences cannot be calculated. This is even more important for $A M$, in which recovery actions to lower primary system pressure are of great interest. Further, if MELCOR is ever to be used for PRA success criteria development, an application that the developers do not currently endorse (F$15)$, such a model would be needed for these calculations also.

3. Representation of Zircalloy oxide shells: The COR model in MELCOR assumes that the fuel fragments or breaks apart when the metal content of a node is reduced to a small, user-input number. Judging from experiment, this is not necessarily the case, and the ability of oxide shells to hold fuel pellets erect for extended periods would impact the heat load imposed on the RCS as well as the timing of debris relocation and vessel failure. The heat load issue applies especially to PWR high-pressure accident sequences in which natural circulation between the core and upper plenum is important. Such phenomena are particularly important for AM studies, in which we are especially concerned 
with estimating the likelihood of hot-leg, steam generator tube, or surge line failure prior to lower head failure.

4. Structure-to-structure radiative hent exchange: MELCOR represents radiation between fluids and structures but does not account for structure-to-structure radiation exchange except in the COR package. This is judged to be of importance in certain sequences likely to be considered in AM studies in which the period of core overheating is greatly prolonged, and the temperatures of hot leg and other structures is of importance.

5. Time-dependent spreading of molten debris as it leaves the RPV: Lack of a model compromises the flexibility and scope of MELCOR evaluations of containment performance for some BWRs. The extent of debris spreading can impact the likelihood of debris quenching, the rate of concrete attack, and the potential for direct interactions between melt and containment structures. Also, a current limitation of MELCOR models for ex-vessel debris behavior (addressing only debris/concrete interactions) precludes mechanistic studies of some important BWR containment failure mechanisms. For example, direct melt-through of a Mark-I containment shell, melt penetration of steel downcomer structure in Mark-II containments, and similar melt/structure interactions in other containment designs, cannot be addressed with the current MELCOR models.

6. Vessel failure modeling, including natural circulation in molten debris pools: MELCOR contains a simple model for the heat load imposed on the lower RPV head from relocated debris. Notably, this model does not include consideration of natural circulation of a molten debris pool in the lower head. Such pools can form if the debris temperature and relocation rate are such as to prevent early failure of bottom head penetrations, or indeed if the plant (like some PWRs) hats no such penetrations. In such a case, the ability to assess the timing and mode of vessel failure, which in turn will have a first-order impact on the containment loads, is compromised by the inability to realistically predict heat loads on the lower head. This affects all accident sequences but is of the greatest concern in high-pressure seyuences in which one is most concerned with predicting the blowdown of the RCS and its effect on the containment.

It is proposed that simple mechanistic models to assess the failure modes of the vessel lower head be developed. The models should include freezing of core material in the instrumentation penetrations and remelting and development of melt release pathways at the penetrations. Transient one-dimensional models should be developed to calculate the conditions leading 10 crust formation, crust growth, and remelting with and without external cooling of the vessel. The molten pool temperature should be calculated as a function of time while allowing for addition of mass into the pool, heat loss by conduction or natural convection to the pool boundaries, and radiative and convective heat loss from the pool free surface to the surrounding structures. A knowledge of the temperature distribution in the steel shell will allow determination of either the thermal or thermo-mechanical failure of the vessel.

\subsection{Missing Models for Mechanistic AM Applications}

As stated above, any proposed application of MELCOR 10 mechanistic AM-related studies places much more difficult burdens on the code than do PRA and other parametric applications. In many cases, a model, which is regarded as second-order for the purpose of computing containment failure timing or source term in unmitigated accident sequences, maly be much more important for assessing the efficacy of recovery stralegies. For this reason, the Committee elected to separately list missing models that could impact mechanistic AM applications, e.g., the prediction of the effects of recovery strategies. In fairness, it should be noted that 
MELCOR's limitations in this area are for the most part also shared by other eodes. Also, as stated earlier, the NRC does not currently sanction use of MELCOR for other than parametric $\triangle M$ studies.

The following phenomena, which are not currently modeled in MLLCOOR, were judged to be necessary for mechanistic AM studies. Some of the phenomena could have an important effect on PRA results also, However, the Commiltee judged that the code's existing parametric capabilities are sufficient for PRA applications for the foreseenble future, Development of some of these models would be seriously compromised by the complexily of the phenomena and limitations in the severe accident knowledge base.

1. Qxidation of steel heal sinks: In high-pressure accident sequences, above-core structures malattain temperatures sufficiently high that oxidation becomes important. This can only be represented in MELCOR if the structures in yuestion are modeled by the COR package instead of the HS package. However, if this is done, other phenomena such as fission product deposition on the structures are then neglected.

2. Multidimensional heal sink model:MELCCOR's heat structure package models heal sinks in only one dimension; that is, only gradients in the throughthickness direction are compuled. This occasionally caluses problems when waler levels atre varying in PRA applications, though a clever user can usually work around this limitalion. For seguences such as attempted recoveries in an AM context, where there is a water source to a volume containing a hot heal sink, the Committee was not as confident that the problems can always be worked around.

3. Pressurizer drainage: In high-pressure PWR sequences, water may remain in the pressurizer for an extended period, even after the RCS side of the surge line uncovers, due to countercurrent limitations on the rate of drainage. MELCOR has no explicit model for this phenomenon. As such, the code may provide inappropriate guidance for the development of $\triangle M$ procedures related to detection of core damage in the RCS and may not impose accurate boundary conditions for core damage processes.

4. Energetic fuel coolime interactions: MELCOR has no model for steam explosions or related phenomena. Consideration of these phenomena is important when mechanistically assessing the deleterious effects of possible recovery strategies in both the RCS (low-pressure sequences) and containment.

5. Milterials interactions. Mlid.COR has no model for chemical interactions between $\mathrm{UO}_{2}$ and zirconium, or Inconnel and zirconium. The Committee felt that this is an important deficiency when assessing accident timing and the efficalcy of in-core recovery actions. It has been stated that a modeling effort in this area is funded to begin in FY 1991.

6. Core reflood and in-core debris suenching: 'To evaluate the potential to avoid RPV failure by reflooding an overheated core or to assess the possibly deleterious elfects of such an action, it is clearly necessary to have a model for credibly predicting the interactions between water and overheated fuel rods or core debris. This involves the ability to predict yuenching rates in the geometries of interest, the shattering of overheated fuel during yuench, the occurrence and effects of clad ballooning, and the possibility of forming a molten pool. None of these processes is currently modeled.

7. Effects of degradation in core geometry on flow: MELCOR does not explicitly model the effects of degradation in core geometry on the flow rate of steam and hydrogen in the various coolant channels. This is another difficult area to treat definitively, but the onset of ballooning or melting will tend to divert flow to other, less damaged areas, especially in PWRs. This can be expected to reduce hydrogen production and fission product release rates, and influence the heating of above-core structures. At present, the user can only construct an ad hoc 
control function that affects the overall flow resistance of the entire core as seen by $\mathrm{CVH}$, not the individual channel or nodal flow resistances.

8. Formation and breach of molten pools of core debris: The TMI aceident suggests that, under some circumstances at least, a crust can form to inhibit the downward progression of the core degradation process. Modeling the formation of such pools and the ultimate failure of the crust is important if the rate and timing of material relocation to the lower plenum are to be accurately estimuted.

\subsection{Conclusions Regarding the Completeness of MELCOR's Modeling Vis-a-Vis the Targeted Applications}

Clearly mechanistic AM studies put very great burdens on MELCOR. In view of the many models that are missing from MELCOR but appear necessary for comprehensive, mechanistic AM studies, in the near term applications of the code must be focussed on rather broad issues in which limited accuracy or purely parametric results are tolerable. The NRC envisions that MELCOR will, in fact, be restricted to such parametric applications. Even parametric studies of the effects of various recovery strategies are compromised by the lack of many missing and important models.

The situation with respect to PRA is less clear cut. If we assume that all the modeling efforts scheduled to begin in FY 1991 are successfully completed, and this cannot be regarded as assured given the inherent difficulties involved, many of the serious modeling omissions will have been addressed. With proper allowance for the phenomena that will remain unmodeled, the code can in our judgment be applied to many accident sequences without the lack of models being of overriding concern. However, here will remain very important classes of accident sequences, i.e., those impacted by the models listed in the first category above which are not funded, in which the code will remain inadequate since it neglects the phenomena which are driving the key figures of merit. It goes without saying that our concerns in this area would be considerably greater if the modeling efforts scheduled to begin in FY 1991 are not expeditiously completed.

\section{REFERENCES}

4-1. F. Elawila, Accident Evaluation Branch, Division of Systems Research, Office of Nuclear Regulatory Research letter about MELCOR objectives and larget applications to B. E. Boyack (November 2, 199()).

4.2. B. E. Boyack and J. Richardson, "Final Minutes of the Fifth MELCOR Peer Review Meeting," Los Alamos National Laboratory Group N-12 letter N-12-91-480) (July 1.5, 1991).

4-3. F. J. Rahn, "LWR Aerosol Containment Experiments (LACE) Project," Electric Power Research Institute report NP-6(194-D) (November 1988). 


\section{O'THER REVIEW FINDINGS}

\subsection{Documentation}

\subsubsection{Background}

The avallability, content, completeness, and quality of documentation is an importunt factor influencing the outcome of a code peer review activity. The Commillee review was based, in large measure, upon the body of the MELCOR document data base presented in Appendix B. More importantly, access to adequate documentation is generally recognized as a prerequisite to effective understanding and application of a complex computer program. In recognition of this need, the NRC has recently provided guidance to organizations involved in the development of software for the NRC's Office of Nuclear Regulatory Reseurch (Ref, 5-1). Documentation of completed software is to consist of four elements, which cinn either be separute volumes or combined, depending upon the size and utility of the code: (1) a code manual, (2) a user's manual, (3) a developmental assessment manual, and (4) an independent assessment manual. The NRC required documentation comprise a useful, although not unique, description of the records needed for a code such as MELCOR.

The code manual is to describe the physical problem, the formulation of the equations that address the problem, the finite-difference and numerical solution method, the structure of the code, the models and/or empirical correlations used including their underlying data base and range of applicability, the applications and limitations of the code, etc.

The user's manual describes to the code user how to prepare the necessary input description for the facility to be modeled. It also provides modeling guidance for different facility configurations, including nodalization where appropriate.

The developmental assessment document is prepared by the code development group as a part of the development process. The documentation includes a description of the basis for and composition of the developmental assessment matrix and comparisons with code calculations.

The independent assessment document contains descriptions of assessment efforts performed external to the code development group on a frozen code, that is, one whose capabilities and structure, including models and correlations, are essentially fixed, but which may still be subject to correction of errors.

\subsubsection{Committee Findings}

Each of the documentation elements identified in Ref. 5-1 exist in one form of MELCOR documentation or another. The body of existing documentation represents a significant and positive accomplishment. The Committee was able, for example, to accomplish a significant portion of its review using the documentation that has already been prepared. The Committee does, however, have concerns about particular aspects of the documentation, and these are detailed in the following paragraphs.

The MELCOR summary report (Ref, 5-2) and phenomenological package reference manuals of Ref. 5-3 cover the elements of the code or theory manual. The Committee determined, however, that the level of detail was less than that reguired to prepare complete findings about the detailed models and correlations using the "Standard of Technical Adequacy" defined in Chapter 1. In absence of the needed documentation of the detailed models and correlations, the Committee obtained the needed information via interrogatories and SNL responses (Refs. 5-4 through 5-12). In some cases, the SNL responses directed Committee members to an already documented source of the requested information. In other cases, however, the Committee felt that the detailed descriptions of the models and correlations were lacking. 'That is, the models in the code were described but the descriptions of pedigree, applicability, and benchmarking were either inadeguate or missing. The NRC has recently requested and received documentition of the models and correlations in its thermal-hydraulic systems codes. The detail in these documents (e,g., Ref, 513) is that which is needed to complete a thorough review for technical adequacy. The Committee recommends that careful consideration be given to producing an equivalent document for the 
MELCOR code. At a minimum, the Committee recommends that all new model development be accompanted by detalled documentation of model pedigree, applicubillty, and fidelity (benchmarking).

A particular area of concern to the Committee is the dispersed nature of model documentation when other computer codes, or parts of other computer codes, have been imported into MELCOR. In several areas, MELCOR documentation simply references the manuals for the parent code without sufficient supporting discussion of which portion(s) of the parent code models have been incorporated anchanged into MELCOR, which have been modifled, and how the imported models have been incorporated into the MELCOR frumework. Examples are the aerosol mechanics models of MAEROS, the core-concrete interaction models of CORCON-MOD2, the exvessel fuel release model from VANESA, and the suppression pool bubble phenomena model from SPARC. The ideal solution would be to incorporate into the MELCCOR documentation a complete description of ench imported physical model. At a minimum, the Committee recommends that references to parent code documentation in the MELCOR documentation be accompanied by clear and specific discussions of how and why the imported models have been selected for MELCOR implementation. The discussion should explicitly identify, by reference to the parent code documentation if necessary, the specific models, equations, applicability discussions, and benchmarking pertaining to use of the imported model(s) in MELCOR.

The MELCOR developmental assessment documentation is sparse, Reference 5-14, as prepared by SNL, is one developmental assessment document. It collects and documents a number of assessment activities for 1986. The abstract of Ref. 5-14 states, "It is intended that a report of this nature be published annually." Since 1986, however, only a few developmental assessment studies have been published (e.g., Refs, 5-15 through 5-17). The Committee recommends that the MELCOR developmental assessment be expanded and the results of the assessment effort be thoroughly documented. Reference 5-18 describes the currently planned MELCOR assessment plan. The Committee recommends that as this or an alternative plan is enacted, careful consideration be given to documentation of the assessments, individually and collectively, so that the lessons learned in the assessment process are incorporated into the MELCOR development effort and and immediately helpful to the user community. This latter objective could be accomplished if each assessment included a section describing modeling code application lessons learned. Independent assessment of MELCOR has been under waly at BNL for several years. Individual independent studies are conducted and documented (e.g., Ref's. 5-19 through 5-22). Independent assessment studies have not been collected, to date, into a summary document. As all developmental assessments and a substantial fraction of the independent assessments are NRC funded, the Committee recommends that all such assessments be documented and collected in a format that promote accessibility and vallue to the user community.

The design of the MELCOR code, with its strong emphasis on user input for modeling both the facility and parametric studies of the many and complex physical processes being examined, places a uniquely difficult burden on the MELCOR user. Effective use of MELCOR demands a knowledgeable user. The Committee believes the design philosophy implemented by SNL carries with it a direct and ongoing responsibility; i.e., that the MELCOR code development staff identify, develop, and document user guidelines and find an effective way to provide these guidelines to the MELCOR user community. SNL, has prepared user guides, which are included as part of Ref. 5-3. However, these guides do not provide enough information. In some cases, defaults recommended in documentation are recognized as being either not workable or not the best choice (Ref, 5-23). The Committee recommends that there be an ongoing effort to remedy this deficiency. In addition, there are many difficult modeling decisions to be made and much of the practical guidance, which helps the user to make these decisions is not readily available. Documentation of practical modeling gudelines is needed. Finally, the ongoing collection of user lessons learned or practical guddelines does not seem to be oceurring. The Commiltee recommends that a structured and ongoing process of collecting, documenting, and distributing practical user guidelines to the MELCOR user community be developed and executed. 


\subsection{Inconsistency in Level of Modeling Detail}

\subsubsection{Background}

The NRC has pursued a strillegy defined to be "two-tier" in developing compuler codes for severe accident simulation and analysis (Ref, 5-24). A collection of "mechanistic" codes has either been developed, or is under development, that addresses selected portions of severe accident behavior in substantial detail. "Integral" computer codes have been and continue to be developed to characterize the entire spectrum of severe accident phenomena with the intent of capturing important synergistic effects (Ref', 5-25). An important application of the integral codes is to PRA studies, which required that these codes be sufficiently fast running to facilitate practical evaluations of numerous accident scenarios and concomitant sensitivity studies. The conflleting attributes of comprehensive modeling and fast running requires application of simpler (i.e., less detailed or parametric) models for many phenomena in the integral codes. One objective, then, of the mechanistic eodes is to provide a mechanism for henchmarking or validating simple models that would be used in the integral codes. The Source Term Code Package (STCP) was the first integral code developed by the NRC: MELCCOR is its designated replacement.

Over the course of MELCOR's development (from 1983 to the present), the conceptual line between the two tiers of codes has blarred. Several models from mechanistic codes were implemented in MELCCOR and, in some aneas, entire mechanistic codes or code modules were entirely absorbed or imported by MELCOR, In many other areas, however, models have been developed for MELCOR that are clearly simpler than those used in corresponding mechanistic codes. Examples of these include models for fission product release from fuel (CORSOR vs FASTGRAS) and fuel melting, and relexation and melting (COR package vs SCDAP).

\subsubsection{Committee Findings}

The Commiltee recognizes and aceepts that decisions were made to adopt mechanistic models (in total or parts) into MLE COR for a variety of reasons, both technical and programmatic. The Committe finds no major lechnical problems that result from the overlap of the two tiers. In fact, the Committee generally endorses an attitude that when practical (i.e., when it does not adversely affect MELCOR's ability to meet its design objectives), including additional physical detail is desirable. Committee recommendations for additional modeling detail are provided in Chapter 5.

Nevertheless, the overlap in model development strategy underscores the importance of Committee findings in all least two other areas, MELCOR validation and documentation. Specific comments in this regand cian be made as follows:

1. For the two-tier stralegy to work effectively, limely demonstration of a simple model's ability to capture important trends and to generate yuantitative results that are within the major uncertainties of mechanistic evaluations is an important means of developing confidence in calculated results. Direct validation of simple models (e.g., against exact or experimental solutions) are suitable alternative approaches but as discussed in Chapter 2 , the Committee finds validation of these two lypes to be yuite limited.

2. Where major portions of a mechanistic code have been imported into MELCOR, it is not sufficient to simply adopt the validation base and model documentation of the parent code as validation and documentation of the corresponding MELCOR model(s). In all current cases where mechanistic codes have been imported into MELCOR, at least some modifications to parent code have been required to accommodate the MELCOR framework. Corresponding changes to the form, range, and diversity of initial and boundary conditions ma alter a model's performance. The Committee notes, therefore, that some level of revalidation (not defined by the Committee) is warranted. Corresponding checks into the completeness and suitability of parent code documentation is also warranted. 


\subsection{Reproducibility}

\subsubsection{Background}

In several discussions, the Committee was briefly exposed to information that sl.ggested different calculated results were obtained for the same problem (and input deck) when MELCOR was run on different computers. Reference 5-23, presentation 3-8, contains results of calculations for the same input deck on a VAX-9000 (a 32-bit machine) and a CRAY X-MP/28 (a 64-bit machine). One example provided was the timing of the first deflagration in a particular control volume; 59,300 s was calculated on the VAX and 38,400 s on the Cray. Lower head penetration was calculated at $18,800 \mathrm{~s}$ on the VAX and $8820 \mathrm{~s}$ on the Cray. Additional results may be found in Ref. 5-26.

\subsubsection{Committee Findings}

Machine-to-machine dependencies are not unusual and have been observed with other large codes. Nevertheless, the Committee concludes that such machine dependencies are of concern. In the final analysis, the user applies a code in the expectation that "the" result will be obtained for a given input deck regardless of the machine used to run the code. Thus, the reality that several results can be obtained depending upon the computer eliminates that expectation and the user is left to ask which of the solutions is "the" solution. The Committee notes that this issue may be another manifestation of the numerics problems discussed in Chapter 2.

\section{REFERENCES}

5-1. B. W. Sheron, "NRC/RES Software Documentation Guidance," US Nuclear Regulatory Commission Division of Systems Research letter to T. Hirons, Los Alamos National Laboratory (April 18, 1991).

5-2. R.M. Summers, R.K. Cole, Jr., E.A. Boucheron, M.K. Carmel, S.E. Dingman, and J.E. Kelly, "MELCOR 1.8.0: A Computer Code for Severe Nuclear Reactor Accident Source Term and Risk Assessment Analyses," Sandia National Laboratories report SAND90-(0364 (NUREG/CR-5531) (January 1991).

5-3. R. M. Summers, "MELCOR Primer, Version 1.8.0," Sandia National Laboratories unnumbered report (February 17, 1989).

5-4. Questions and Answers, "Heat Structure Thermal Response"

Questions by V. Dhir (November 8, 1990)

Answers by M. K. Carmel, Sandia National Laboratories, Second MELCOR Peer Review Meeting, Albuquerque, New Mexico (November 5-7, 1990).

5-5. Questions and Answers, "Core/Concrete Interactions"

Questions by V. Dhir (November 12, 1990)

Answers by R. K. Cole, Sandia National Laboratories, Second MELCOR Peer Review Meeting, Albuquerque, New Mexico (November 5-7, 199()). 
5-6. Questions and Answers, "Heat Structures (HS) Package" and "Core Heatup and Degradation (COR) Package"

Questions by R. Viskanta, Letter to B. E. Boyack Re: Questions Concerning MELCOR to be addressed by SNL at the November 5-7, 1990 Committee Meeting. (September 24, 1990)

Answers by M. K. Carmel, Sandia National Laboratories, Second MELCOR Peer Review Meeting, Albuquerque, New Mexico (November 5-7, 1990)

Answers, "Core Heatup and Degradation (COR) Package" by R. M. Summers, Sandia National Laboratories, Second MELCOR Peer Review Meeting, Albuquerque, New Mexico (November 5-7, 1990).

5-7. Questions and Answers, "MELCOR Hydrodynamics" and "Core-Concrete Models" Questions by M. Khatib-Rahbar, Letter to B. E. Boyack Re: Preliminary Questions on MELCOR Hydrodynamics and Core-Concrete Models (October 2, 1990)

Answers, "Responses to Dr. Khatib-Rahbar's comments on MELCOR CVH/FL Package by R. K. Cole, Sandia National Laboratories, Second MELCOR Peer Review Meeting, Albuquerque, New Mexico (November 5-7, 1990)

Answers, "Responses to Dr. Khatib-Rahbar's comments on MELCOR CAV package," by R. K. Cole, Sandia National Laboratories, Second MELCOR Peer Review Meeting, Albuquerque, New Mexico (November 5-7, 1990).

5-8. Questions and Answers, "Core Modeling" and "Material Properties"

Questions by T. J. Haste, Letter to B. E. Boyack Re: MELCOR Peer Review-Second Meeting (September 27, 1990)

Answers, "Answers to Tim Haste's Questions on COR Package Modeling" by R. M. Summers, Sandia National Laboratories, Second MELCOR Peer Review Meeting, Albuquerque, NM (November 5-7, 199())

Answers, "Responses to Additional Haste Questions on COR Package Modeling" by R. M. Summers, Third MELCOR Peer Review Meeting, Albuquerque, New Mexico (January 11, 1991).

5-9. Questions and Answers, "BUR Package"

Questions by M. Kenton, Second MELCOR Peer Review Meeting (November 5-7, 1990)

Answers, "Responses to Kenton on BUR Package Modeling" by R. M. Summers and S. Dingman, Sandia National Laboratories, Third MELCOR Peer Review Meeting, Albuquerque, New Mexico (January 14-16, 1991).

5-10. Questions and Answers, "SPR and FCL Packages"

Questions by M. Kenton, Second MELCOR Peer Review Meeting (November 5-7, 1990)

Answers, "Responses to Kenton Questions on SPR and FCL Package Modeling" by R. M. Summers, Sandia National Laboratories, Third MELCOR Peer Review Meeting, Albuquerque, New Mexico (January 14-16, 1991).

5-11. Questions and Answers, "HS and COR Packages"

Questions by R. Visanta, letter to B. E. Boyack (December 27, 1990)

Answer, "Responses to Additional Viskanta Questions on HS Package Modeling" by R. M. Summers, Sandia National Laboratories, Third MELCOR Peer Review Meeting, Albuquerque, New Mexico (January 11, 1991).

Answer, "Responses to Additional Viskanta Questions on COR Package Modeling" by R. M. Summers, Sandia National Laboratories, Third MELCOR Peer Review Meeting, Albuquerque, New ivexico (january i i, i99i). 
5-12. Questions and Answers, "RN Package"

Questions by R. S. Denning, Letter to B. E. Boyack (October 16, 1990)

Answers, "Issues to be Addressed for RN Package" by E. Boucheron, Sandia National Laboratories, Third MELCOR Peer Review Meeting, Albuquerque, New Mexico (January 14-16, 1991).

5-13. D. R. Liles, J. W. Spore, T. D. Knight, R. A. Nelson, M. W. Cappiello, K. O. Pasamehmetoglu, J. H. Mahaffy, L. A. Guffee, H. J. Stumpf, P. J. Dotson, R. G. Steinke, P. R. Shire, S. E. Greiner, and K. B. Sherwood, "TRAC-PF1/MOD1 Correlations and Models," Los Alamos National Laboratory report LA-11208-MS (NUREG/CR-5069) (December 1988).

5-14. C.D. Leigh, Editor, "MELCOR Validation and Verification; 1986 Papers," Sandia National Laboratories report SAND86-2689 (NUREG/CR-4830) (March 1987)

5-15. E.A. Boucheron and J.E. Kelly, "MELCOR Analysis of the TMI-2 Accident," Transactions of the American Nuclear Society and the European Nuclear Society 1988 International Conference on Nuclear Fission: Fifty Years of Progress in Energy Security and Topical Meeting on TMI-2 Accident: Materials Behavior and Plant Recovery Technology, Vol. 57, p. 435-436, Washington, DC, Sandia National Laboratories paper SAND88-1258C (October 30-November 4, 1988).

5-16. G. M. Martinez, "MELCOR Calculations of ISP28 SFD PHEBUS Test: B9+," Sandia National Laboratories unnumbered report to B. Adroguer (December 14, 1990).

5-17. S. Dingman, "MELCOR Blind Calculations for HDR Tests 31.4 and 31.5," Sandia National Laboratories Thermal/Hydraulic Analysis Division document (undated).

5-18. L. N. Kmetyk, "MELCOR Assessment Plan," Sandia National Laboratories draft document (March 1, 1991).

5-19. I.R. Madni, "MELCOR Simulation of the PBF Severe Fuel Damage Test 1-1," AICHE Symposium Series, Proceedings of the 26th National Heat Transfer Conference, Philadelphia, Pennsylvania (1989).

5-20. M.I. Robertson, "MELCOR-A Modelling View and Calculations for Phase 1 of the BMC-F2 Experiment," United Kingdom Atomic Energy Authority, Safety and Reliability Directorate report PWR/SAWG/P(90)578, PWR/FPERG/P(90)108 (May 1990).

5-21. I. K. Madni, "MELCOR Modeling of the PBF Severe Fuel Damage Test 1-4," Brookhaven National Laboratory document BNL-NUREG-44503 (1990).

5-22. I. K. Madni and X. D. Guo, "MELCOR Simulation of the Full-Length -Temperature 2 Experiment," Brookhaven National Laboratory Safety Integration Group report (1991).

5-23. B. E. Boyack and I. Richardson, "Final Minutes of the Third MELCOR Peer Review Meeting," Los Alamos National Laboratory Group N-12 letter N-12-91-171 (March 20, 1991).

5-24. F. Eltawila, Accident Evaluation Branch, Division of Systems Research, Office of Nuclear Regulatory Research letter to B. E. Boyack (November 2, 199()).

5-25. "Revised Severe Accident Research Program Plan," U. S. Nuclear Regulatory Commiscion document NIIRFGi-1365 (August 1989). 
5-26. S. L. Chan, H. P. Isaak, U. Schmocker, and M. Khatib-Rahbar, "Characterization of Severe Accidents Using MELCOR: A Perspective," Swiss Federal Nuclear Safety Inspectorate, Presentation at the Cooperative Severe Accident Research Program SemiAnnual review meeting, Bethesda, Maryland (May 6-10, 1991). 


\section{RECOMMENDATIONS}

The MELCOR Peer Review Committee has determined that recommendations in five areas are appropriate: (1) MELCOR numerics, (2) models missing from MELCOR Version 1.8.1, (3) existing MELCOR models needing revision, (4) demonstrated MELCOR adequacy, and (5) documentation. These recommendations are the summary outcome of the Committee's review efforts and have resulted from the screening of a larger set of issues detailed in the body of this report.

The MELCOR Peer Review Committee recognizes that resources for MELCOR development, revision, and enhancement are and will continue to be limited. Therefore, we have made a concerted effort to prioritize our findings. In this chapter, we present only those recommendations that we feel are necessary for MELCOR to successfully fulfill its design objectives and satisfactorily perform in its targeted applications. We have focused on the PRA application. In identifying our priority recommendations, we have considered the importance of each identified deficiency relative to the potential impact on (1) the time of containment failure, and (2) the magnitude of fission product release to the environment. We did not consider the AM application separately. We assumed that a code suitable for the PRA application would also be suitable for the type of AM studies endorsed by NRC management, i.e., those focused on scoping studies (Ref. 6*1).

\subsection{MELCOR Numerics}

The Committee detailed its concerns in Chapter 3. The Committee concluded that code numerics are the source of a primary concern regarding the technical adequacy of the code. The Committee's concern about the MELCOR numerics arises from several sources including sensitivity to input parameters, noding, and time step. For example, during the course of the MELCOR Peer Review, the results of several time-step sensitivity studies were made available to the Committee. These studies indicated that convergence to limiting values is not currently guaranteed as the time step decreases. In fact, key quantities vary erratically as the time step is changed. In some cases, such as those in which substantial variation in results occurs because of changes in maximum time step, it is obvious that the observed parameter sensitivity is an artifact of the numerics. This leads to a concern that sensitivities that are observed for other, more physically based parameters, might also be at least partially the result of numerics. Because sensitivity analyses are central to most severe accident studies, failure to resolve this matter seriously compromises MELCOR's use for its targeted applications. The Committee concludes that an improved understanding of parameter sensitivities is important and that correction of the MELCOR numerics problems should be considered to be a high-priority activity.

\subsection{Models Missing from MELCOR Version 1.8.1}

After completing its screening process, the Committee concluded that missing models could naturally be divided into three categories: (1) missing models necessary for PRA applications, (2) missing models that, while less important, would significantly improve MELCOR's capability for PRA and parametric accident management studies, and (3) missing models needed if MELCOR were ever to perform mechanistic accident management calculations. As only the first of the three categories satisfies the screening logic, the Committee concludes that inclusion of models for the following phenomena should be given the highest priority:

- PWR primary system natural circulation within components of the primary coolant system (e.g., recirculation within the vessel, hot-leg recirculation, and steam generator recirculation):

- high-pressure melt ejection and direct containment heating;

- ice condenser behavior;

- nonexplosive interactions between debris and water; 
- $\quad$ fission product vapor scrubbing;

- additional reactor cooling system fission product deposition processes; and

- fission product reactions with surfaces.

Further descriptions of these missing models are provided in Section 4.3 in Chapter 4 . The Committee notes that funded model development activities are currently either planned or under way for part of the PWR primary system natural circulation issue, the high-pressure melt ejection and direct containment heating model, and the ice condenser model.

\subsection{Existing MELCOR Models Needing Revision}

The Committee's bottom-up review of the MELCOR phenomenological packages identified a number of individual models of concern (Category 4 and 5 models as defined in Chapter 1). The lead reviewers for each MELCOR phenomenological package identified the three or four Category 4 or 5 models believed to have the most significant adverse affect on the ability of MELCOR to attain its design objectives and satisfy its targeted applications. The collection of these models was further reviewed, first by a screening subgroup of the Committee and then by the full Committee. The collection of Category 4 and 5 models reviewed and individual models ranked as either very important, important, or less important. The Committee recommends that the following issues ranked as "very important" be corrected or issues be resolved with the highest priority.

- An evaluation should be made to determine whether the suspended water condensation/evaporation model used in the CVH package is implemented adequately. Currently, condensation is treated independently in the CVH package from the calculations of aerosol particle growth and deposition in the RN package. The condensation growth of aerosols is in competition with wall surfaces for available water and hence is not an independent process. Because the size change for particles (droplets) as a result of condensation may have an overriding effect on aerosol deposition rates, the correct allocation of condensing water is a controlling factor. Therefore, the acceptability of the current model should be demonstrated (see Appendix G for further details).

- Inconsistencies in treatment of chemical reactions between CORCON and VANESA should be resolved, and improvements should be made to the CORCON/MOD2 phase diagrams. Consolidation of CORCON and VANESA into a unified code as currently planned for CORCON/MOD3 is desirable. The Committee did not review CORCON/MOD3 models. However, based on a brief presentation to the Committee by SNL, it appears that this code has the potential for remedying most of these concerns. A peer review of CORCON/MOD3 models would be desirable before its implementation into MELCOR (for the CAV package, see Appendix $\mathrm{H}$ for details).

- The model for steam condensation on containment heat sinks (mass transfer) should be revised. With the existing model, steam condensation rates predicted for conditions when the thermal resistance of the structure on which condensation occurs does not dominate will be in serious error. The model used for condensation in the presence of noncondensables is seriously flawed (see Appendix E for further details).

- The pool scrubbing model for aerosols is largely derived from previously available models but assumes spherical bubble shapes while correcting for bubble flattening by using coefficients derived from comparisons to other models. Impaction of particles from steam/air jets entering the pool would be expected to provide significant deposition for larger particles but the effect is ignored in the current model. Decontamination factors computed with the current model are quite low in comparison with other models and the existing data base (see Appendix $\mathrm{G}$ for further details). 


\subsection{Need for Expanded MELCOR Assessment}

The Committee concluded that the ability of MELCOR to calculate severe accident phenomena is not sufficiently demonstrated. Such a demonstration would be based on a documented collection of (1) sensitivity studies, (2) benchmarking activities using experimental data, and (3) code-to-code assessments.

The Committee has concluded that review of a comprehensive set of well-defined and executed sensitivity analyses is an important and necessary component of the effort to determine technical adequacy. Unfortunately, this comprehensive set of MELCOR sensitivity analyses does not presently exists. Rather, a sparse set of sensitivity studies exists, and several of these studies only became available during the conduct of the MELCOR Peer Review effort. Two categories of sensitivity analyses are of particular interest and importance in assessing code technical adequacy. First, sensitivity studies to improve understanding of physical processes in an integral context are needed. Second, time-step and noding sensitivity studies are believed to be important.

By any measure, the MELCOR integral benchmarking effort is extremely small. The Committee has concluded that a more comprehensive and ongoing integral assessment program is needed. This program would (1) provide an increased flow of needed information to the code developers regarding code deficiencies, (2) promote the development of user guidelines, and (3) improve the level of knowledge regarding the performance of the code relative to its targeted applications.

While encouraging an increasing pace to the integral assessment effort, the Committee emphasizes the importance of maintaining an overall perspective on the role of integral benchmarks $v s$ the qualification of individual models. A complete assessment of the technical adequacy of an integral code considers both the component parts (bottom-up review) and the adequacy of the integrated coded package (top-down review). The detailed or bottom-up review focuses on the pedigree, applicability, and fidelity of the individual models and correlations. The Committee feels that there is limited value in assessing the integral performance of the code if key building-block models or correlations are seriously flawed.

Although code-to-code comparison cannot and should not displace or replace code benchmarking efforts against test data, the Committee concludes that such compi risons can provide useful supplementary insights. For severe accident phenomena, their value lies not in the absolute, i.e., what is right and what is wrong, but in focusing attention on what is different. When properly conducted, such efforts can identify and highlight, for example, models that are either incorrect, not applicable for the phenomena being analyzed, or otherwise deficient. The Committee recommends that such comparison activities continue in the future.

\subsection{Documentation}

Although the Committee concludes that the body of existing MELCOR documentation (theory manual, user guides, and code assessment reports) represents a significant and positive accomplishment, changes and enhancements to the MELCOR documentation are recommended.

The NRC has recently requested and received documentation of the models and correlations in its thermal-hydraulic systems codes. The detail in these documents (e.g., Ref. 6-2) is that which is needed to support knowledgeable use of the code. The Committee recommends that careful consideration be given to producing an equivalent document for the MELCOR code. At a minimum, the Committee recommends that all new model development be accompanied by detailed documentation of model pedigree, applicability, and benchmarking.

A particular area of concern to the Commillee is the dispersed nature of model documentation when other computer codes, or parts of other computer codes, have been imported into MELCOR. The ideal solution would be to incorporate into the MELCOR documentation a complete description of each imported physical model. At a minimum, the Committee recommends that references to parent cole documentation in the MELCCOR documentation be accompanied by a clear and specific discussions of how and why the imported models have been selected for MELCOR implementation. 'The discussion should explicitly identify, by reference to the parent 
code documentation if necessary, the specific models, equations, applicability discussions, and benchmarking pertaining to use of the imported model(s) in MELCOR.

'The MELCOR developmental assessment documentation is sparse. The Committee recommends that the MELCOR developmental assessment be expanded and that the results of the assessment effort be thoroughly documented. Reference 6-3 describes the currently planned MELCOR assessment plan. The Committee recommends that as this or an alternative plan is enacted, careful consideration be given to documentation of the assessments, individually and collectively, so that the lessons learned in the assessment process are incorporated into the MELCOR development effort and and immediately helpful to the user community. This latter objective could be accomplished if ench assessment included a section describing modeling code application lessons learned. Independent assessment studies have not been collected, to date, into a summary independent assessment document. As all developmental assessments and a substantial fraction of the independent assessments are NRC-funded, the Committee recommends that all such assessments be documented and collected in a format that promotes accessibility and value to the user community.

The design of the MELCOR code, with its strong emphasis on user input for modeling both the facility and parametric studies of the many and complex physical processes being examined, places a uniquely difficult burden on the MELCOR user. Selection of appropriate values for the various parameters used in the phenomenological models is crucial because the representation of physical processes can be markedly influenced by parameter selections. Effective use of MELCOR demands a knowledgeable user. 'The Committee believes the design philosophy implemented by SNL carries with it a direct and ongoing responsibility; i.e., that the MELCOR code development staff identify, develop, and document user guidelines and find effective ways to provide these guidelines to the MELCOR user community. The Committee recommends that there be an ongoing effort to remedy this deficiency. In addition, there are many difficult modeling decisions to be made, and much of the practical guidance that helps the user to make these decisions is not readily available. Documentation of practical modeling guidelines is needed. Finally, the ongoing collection of user lessons learned or practical guidelines does not seem to be occurring. The Committee recommends that a structured and ongoing process of collecting, documenting, and distributing practical user guidelines to the MELCOR user community be developed and executed.

\section{REFERENCES}

6-1. B. E. Boyack, "Actions From 5th MELCOR Peer Review Meeting," Los Alamos National Laboratory Group N-12 letter N-12-91-378 (June 3, 1991).

6-2. D. R. Liles, J. W. Spore, T. D. Knight, R. A. Nelson, M. W. Cappiello, K. O. Pasamehmetoglu, et al., "TRAC-PF1/MOD1 Correlations and Models," Los Alamos National Laboratory report LA-11208-MS (NUREG/CR-5069) (December 1988).

6-3. L. N. Kmetyk, "MELCOR Assessment Plan," Sandia National Laboratories draft document (March 1, 1991). 


\section{Acknowledgments}

The MELCOR Peer Review Committee wishes to acknowledge the efforts of many individuals who have contributed to the success of this effort. C. Alexander of Battelle Columbus Laboratories contributed the review materials related to the MELCOR chemistry models. Several individuals from Los Alamos National Laboratory also contributed significantly to this effort. D. Liles assisted in the review of the CVH and FL phenomenological packages and completed several assessment problems for these packages. J. Spore and T. Chen assisted in the review of the COR phenomenological package. J. Richardson served as recorder for the formal meetings and contributed to the technical discussions. K. Ames edited this report. G. Mirabal was responsible for the document processing activities. We also wish to acknowledge the excellent support and assistance received from the MELCOR development staff at Sandia National Laboratories. In addition to hosting four of the Committee meetings, the MELCOR development staff members were fully responsive to the requests for information and documentation. Finally, the MELCOR development staff showed grace under pressure throughout the review process. This is a significant challenge given that code development frequently engenders feelings some what akin to parenthood. 


\title{
APPENDIX A
}

\author{
Members of the MELCOR Peer Review Committee
}

\section{B. E. Boyack}

Brent Boyack is the Chairman of the MELCOR Peer Review Committee. He holds a joint appointment at the Los Alamos National Laboratory where he is leader of the Terrestrial Reactor Technology Section within the Reactor Design and Analysis Group and leader of the Thermal-Hydraulics/Accident Analysis Team within the New Production Reactor, Safety Technology Office. He has published extensively on light water reactor thermal-hydraulic code. assessments, code uncertainty quantification, gas-cooled thermal and fast reactor fluid dynamics and heat transfer, and computer model development. He has participated in NRC review groups focusing on the quantification of uncertainty in the NRC-developed thermal-hydraulics code TRAC-PF1/MOD1, quantification of uncertainty in the NRC-developed thermal-hydradic code RELAP5/MOD3, the Severe Accident Scaling Methodology development, and the Expert Panel review of a proposed AP600 Integral Test Facility. Before accepting employment with Los Alamos National Laboratory, he was a Senior Staff Member at General Atomic Company where he held technical and management positions working in the High-Temperature Gas-Cooled Reactor and the Gas-Cooled Fast Reactor programs. He was also employed by the Boeing Company where he applied numerical methods to the solution of engineering analysis problems. He holds a Ph.D. in Mechanical Engineering from Arizona State University and is a registered Professional Engineer in California.

\section{Khatib-Rahbar}

Mohsen Khatib-Rahbar is the President of Energy Research, Inc., of Rockville, Maryland. His research focuses on nuclear reactor safety and probabilistic risk assessment. He has published extensively on severe accidents, source terms, methods for uncertainty analysis, consequence assessment, thermal-hydraulics, and numerical methods. He has also developed computer models for simulation of thernal-hydraulics and neutronics transients in light water reactors and liquid metal fast-breeder reactors. Before starting Energy Research, Inc., he was a staff scientist at Brookhaven National Laboratory where he managed programs dealing with level 2/3 PRA reviews, verification and benchmarking of STCP and MELCOR, source term uncertainties (QUASAR), Zion/Draft NUREG-1150, and regulatory implications of new source terms. He was a visiting scientist at Gesellschaft für Reaktorsicherheit (GRS) in Germany (1982), and the NRC Office of Nuclear Regulatory Research (1988-1989). He is currently a consultant to the US Department of Energy, the US Nuclear Regulatory Commission, the Swiss Federal Nuclear Safety Inspectorate, and several national laboratories and private organizations. He holds a Ph.D. in Nuclear Science and Engineering from Cornell University.

\section{Y.K.Dhir}

Vijay K. Dhir is a Professor of Engineering and Applied Science in the Mechanical Aerospace and Nuclear Engineering Department of the School of Engineering and Applied Science at the University of Califormia at Los Angeles (UCLA). During the past 17 years he has done both basic and applied research in the thermal sciences and in energy conversion systems. His basic research is on the phenomenological studies of phase change heat and mass transfer. This research includes experimental and analytical investigations of pool and forced flow boiling under saturated and subcooled conditions, two phase flow in porous media, film condensation, simultaneous melting and condensation under steady state and transient conditions, and evaporation. In the applied areas he has worked on safety and thermal hydraulics of fission and fusion nuclear power 
reactors. The studies have included reflood heat transfer, degraded core heat transfer and fluid flow phenomenology, core-concrete interactions, natural convection and stratification in liquid metal cooled reactors, melting, freezing, and plugging of coolant channels in transicnt overpower accidents. In the past he has served on various Department of Energy review panels and has been a consultant to Atomics International, Canoga Park, in support of the design efforts for a pool-type fast-breeder reactor with inherent safety characteristics. He has also acted as a consultant to the National Bureau of Standards, Science Applications International Corporation, Battelle Northwest Laboratories, EG\&G, Idaho Inc., General Electric, Electric Power Research Institute, and Pickard, Lowe and Garrick. Dr. Dhir obtained his Ph.D. in Mechanical Engineering from the University of Kentucky, Lexington, and has published more than 10() papers in various national and international journals and conference proceedings. At UCLA, he teaches courses in heat transfer, thermodynamics, and nuclear reactor thermal-hydraulic design.

\section{A. Gieseke}

James A Gieseke is Manager of the Chemical Measurements and Methods Department at Battelle Memorial Institute, Columbus, Ohio. Over the past 27 years at Battelle his research has focused on issues concerned with the dynamics of aerosol particles. He is expert in disciplines including particle formation, interaction of particles with gases and with other particles, design of particle sampling and collection equipment, the control and dispersion of particles within buildings and equipment, and the modeling of vapor and aerosol transport and deposition. He managed and participated in development of the HAARM, QUICK, QUICK-M, and TRAP-MELT codes for fission product transport in primary systems and containments as well as the CORSOR code for fission product release from fuel. He was also responsible for development of the Source Term Code Package. He managed the pioneering integrated accident analyses for source term estimation as teported in BMI-2104, served as member of the Nuclear Energy Agency's Committee on the Sarety of Nuclear Installation (NEA/CSNI) expert group on nuclear aerosols, and served on the NRC's Expert Panel dealing with Source Term Issues for NUREG-1150). He holds a Ph.D. in Chemical Engineering from the University of Washington, Seattle.

\section{T._._. Haste}

Tim Haste is a senior scientist in the Reactor Safety Studies Department, Safety and Performance Division, AEA Reactor Services, AEA Technology, Winfrith, United Kingdom. He is currently engaged in analysis of early phase melt progression in PWR systems, is Project Manager for UK activities involving MELCOR and SCDAP/RELAP5, and is collaborating actively with national laboratories in the US, France, and Germany. He is presently Chairman of the UK SCDAP/RELAP5 User's Group. Before his appointment at Winfrith, he worked for 10 years at the Springfields Laboratories of AEA Technology, specializing in theoretical analysis of fuel performance in AGR and PWR systems under normal and design basis LOCA conditions, and in thermophysical properties of reactor materials. One year in this period was spent as a visiting scientist at the OECD Halden Reactor Project, Norway. He was a coauthor of the UK Zircalloy Data Manual. Before working at Springfields, he researched at Harwell into Doppler broadening in fast reactors. Dr. Haste maintains a general interest in the water reactor fuels area, acting as a referee for papers and reviews in international journals and conferences, and contributing regularly in these areas. After graduating in theoretical physics from Cambridge University, UK, he obtained a Ph.D. in Nuclear Science from Oxford University, UK. He is a Fellow of the UK Institute of Mathematics, a Member of the UK Institute of Physics (Chartered Physicist), and a Member of the British Nuclear Energy Society. 


\section{M.A. Kenton}

Marc Kenton is President of Gabor, Kenton and Associates, Inc (GKA). His activities there focus on the deterministic analysis of severe accidents in light water reactors, mainly as part of the Individual Plant Examination process. This work includes the analysis of specific accident transients, the development and validation of analytical models, the preparation of guidance for the performance of severe accident analyses by industry, and the education of engineers at nuclear utilities. He is also actively involved in the development and validation of models for advance light water reactors for the Department of Energy. Before co-fouriding GKA, Kenton was the manager of methods development at Fauske and Associates, Inc. In this capacity, he was the architect of the Modular Accident Analysis Program (MAAP), a widely used severe accident analysis computer code, the lead developer of the PWR version of MAAP, and a key contributor to the industrysponsored IDCOR program. He holds a Ph.D. in Nuclear Science and engineering from Cornell University and served on the staff of Admiral H. G. Rickover in the Naval Reactors program.

\section{M.T.Leonard}

Mark T. Leonard is a Senior Staff Engineer and European Technical Liaison for Science Applications International Corporation (SAIC). In this capacity, he serves as the local technical representative and director of reactor safety programs for SAIC's customers in Europe through an office in the Netherlands. Mr. Leonard has experience in the assessment and practical application of large thermal-hydraulic computer codes such as TRAC-BF1 and RELAP5 and participated in the development of the Source Term Code Package (STCP). Before joining SAIC, he managed the Nuclear Systems Group at Battelle where he directed efforts to provide severe accident progression analyses and fission product release calculations for use in NUREG-1150. Recently, he directed a 2-year program for Savannah River Laboratory and developed a wide spectrum of core melt progression models for U-Al fueled, heavy water reactors. The MELCOR code was used as a starting point and as general framework for the new models. Mr. Leonard is currently a consultant for N. V. KEMA in Holland and Gesellschaft fur Reaktorsicherheit (GRS) in Germany. He holds a Masters Degree in Nuclear Engineering from Massachusetts Institute of Technology.

\section{R. Yiskanta}

Raymond Viskanta is W.F.M. Goss Distinguished Professor of Engineering at Purdue University. His research focuses on heat transfer in buoyancy-driven flows, solid-liquid phasechange, radiative transfer in participating media, and combined conduction-radiation as well as convection-radiation heat transfer. Dr. Viskanta has been a Springer and Visiting Professor at the University of California, Berkeley, and a Guest Professor at the Technical University of Munich and at the Tokyo Institute of Technology. Before accepting employment with Purdue University, he was a mechanical engineer at Argonne National Laboratory working in the Reactor Engineering Division. He has served as a consultant on heat transfer and thermal-hydraulics to a number of national laboratories and industry and was a member of the Peer Review Panel on the Draft Reactor Risk Reference Document, NUREG-1150. He is a consultant to the PRA Subcommittee of the US Department of Energy Advisory Committee on Nuclear Facility Safety. He is the Technical Editor of the ASME Journal of Heat Transfer, serves on advisory editorial boards of several journals, and is an author of over 300 journals and publications on heat transfer, thermal sciences, and radiative heat transfer. He holds a Ph.D. in Mechanical Engineering from Purdue University and is a member of the U.S. National Academy of Engineering. 


\section{APPENDIX B}

\section{MELCOR Document Data Base}

This data base is a reference to the documents that were used to perform the MELCOR Peer Review. The documents referred to in this data base are not necessarily accessible to readers.

\section{Beference Reports}

A1. G. G. Weigand, Project Leader, "Thermal-Hydraulic Process Modeling in Risk Analysis: An Assessment of the Relevant Systems, Structures, and Phenomena," Sandia National Laboratories report SAND84-1219 (NUREG/CR-3986) (August 1984).

A2. S. R. Greene, "Realistic Simulation of Severe Accidents in BWRs-Computer Modeling Requirements," Oak Ridge National Laboratory report ORNL/TM-8517 (NUREG/CR2940) (April 1984).

A3. R. M. Ostmeyer, "An Approach to Treating Radionuclide Decay Heating For Use in the MELCOR Coije System," Sandia National Laboratories report SAND84-1404 (NUREG/CR-4169) (May 1985).

A4. "Fission Product Behavior During Severe LWR Accidents: Modeling Recommendations for the MELCOR Code System," from D. A. Powers, "Volume I: Fission Product Release from Fuel," Sandia National Laboratories document SAND85-2743 (NUREG/CR-4481) (September 1988). C. D. Leigh and J. L. Sprung, "Volume 2, Chapter 5, Fission Product Transport and Deposition Including Vapor Condensation and Aerosol Agglomeration," Sandia National Laboratories draft report (undated).

A5. F. Gelbard, "MAEROS User Manual," Sandia National Laboratories report SAND800822 (NUREG/CR-1391) (December 1982).

A6. R. K. Cole, Jr, D. P. Kelly, and M. A. Ellis, "CORCON-MOD2: A Computer Program for Analysis of Molten-Core Concrete Interactions," Sandia National Laboratories report SAND84-1246 (NUREG/CR-3920) (August 1984).

A7. D. A. Powers, J. E. Brockmann, and A. W. Shiver, "VANESA: A Mechanistic Model of Radionuclide Release and Aerosol Generation During Core Debris Interactions with Concrete," Sandia National Laboratories report SAND85-1370 (NUREG/CR-4308) (September 1985).

A8. S. E. Dingman, A. L. Camp, C. C. Wong, D. B. King, and R. D. Gasser, "HECTR Version 1.5 User's Manual," Sandia National Laboratories report SAND86-0101 (NUREG/CR-4507) (February 1986).

A9. C. D. Leigh, Ed., "MELCOR Validation and Verification; 1986 Papers," Sandia National Laboratories report SAND86-2689 (NUREG/CR-4830) (March 1987).

A 10. S. W. Webb, "A Fully-Coupled Model for Aerosol Removal Mechanisms in Rising Bubbles," Sandia National Laboratories draft report SAND86-2940 (NUREG/CR-5091) (April 1990). 
A11. L. A. Miller, G. D. Wyss, D. M. Kunsman, S. E. Dingman, E. A. Boucheron, M, K. Carmel, and C. J. Shaffer, "N Reactor Probabilistic Risk Assessment Supporting Calculations," Sandia National Laboratories report SAND89-2101 (June 1990), Vol. 1.

A12. S. E. Dingman, J, E. Kelly, C. J. Shaffer, A. C. Payne, and M. K. Carmel, "MELCOR Arialyses for Accident Progression Issues," Sandia National Laboratories draft report SAND89-0072 (NUREG/CR-5331) (to be published).

A13. R. M. Summers, R, K. Cole, Jr., E. A. Boucheron, M. K. Carmel, S. E. Dingman, and J. E. Kelly, "MELCOR 1.8.0: A Computer Code for Severe Nuclear Reactor Accident Source Term and Risk Assessment Analyses," Sandia National Laboratorles SAND90)0364 (NUREG/CR-5531) (January 1991).

A14. R. M. Summers, "MELCOR Primer, Version 1.8.0," Sandia National Laboratories document (February 17, 1989). Primer includes the following sections:

MELCOR Architecture Programmer's Guide

Machine Dependent Code in MELCOR

MELCOR/MELGEN

Users' Guides

Burn Package

Cavity Package

Control Function Package

Core (COR) Package

Control Volume Hydrodynamics Package

Decay Heat Package

Fan Cooler (ESF/FCL) Package

External Data File Package

FDI Package

Flow Path

HS Package

Material Properties

MELPLT Users' Guide

Noncondensible Gas EOS

Radionuclide Package

Containment Sprays Package

Tabular Function

Transfer Process

Reference Manuals

Burn Package

Core (COR) Package

Thermal Hydraulic Packages (CVH and FL)

Control Volume Thermodynamics Package (CVT)

Heat Structure (HS) Package

Radionuclide Package

Fan Cooler (ESF/FCL) Package

A15. M. H. Kim, M. D. Oh, G. A. Moses, and M. L. Corradini, "MFLCOR Fuel Dispersion Interactions Model," University of Wisconsin, Reactor Safety Research, Nuclear Engineering Department document (November 1986).

A16. C. C. Wong, "HECTR Analyses of the Nevada Test Site Premixed Combustion Experiments," Sandia National Laboratories report SAND87-0956 (NUREG/CR-4916) (November 1988). 
A17. C. C. Wong, "A Standard Problem for HECTR-MAAP Comparison: Incomplete Burning," Sandia National Laboratories report SAND87-1858 (NUREG/CR-4993) (August 1988).

A18. G. M. Martinez, "ME:COR Calculations of ISP28 SFD PHEBUS Test: B9+," Sandia National Laboratories letter report to B. Adroguer (December 14, 1990).

A19. L. N. Kmetyk, "MELCOR Assessment Plan," Sandia National Laboratories draft document (March 1, 1991).

\section{Published SNI Papers}

B1. J. L. Sprung, D. C. Aldrich, G. G. Weigand, and M. A. Cunningham, "Overview of the MELCOR Risk Code Development Program," Proceedings of the International Meeting on Light Water Reactor Severe Accident Evaluation, Vol. 2, p. 10.1-1, Cambridge, Massachusetts (August 28-September 1, 1983).

B2. G. G. Weigand, "Thermal-Hydraulic Process Modeling in Risk Analysis: An Assessment of the Required Phenomena," Proceedings of the International Meeting on Light Water Reactor Severe Accident Evaluation, Vol. 2, p. 17.1-1, Cambridge, Massachusetts (August 28-September 1, 1983).

B3. A. R. Taig, C. D. Leigh, D. A. Powers, J. L. Sprung, J. C. Cunnane, H. I. Avci, P. Baybutt, J. A. Gieseke, and T. Margulies, "Fission Product Behavior Modeling in Risk Analysis-An Assessment of the Relevant. Phenomena," Proceedings of the International Meeting on Light Water Reactor Severe Accident Evaluation, Vol. 1, p. 2.5-1, Cambridge, Massachusetts (August 28-September 1, 1983).

B4. S. W. Webb, "Coupling of Aerosol Removal Mechanisms in Rising Bubbles," T-ansactions of the American Nuclear Society 22, pp. 525-527, Reno, Nevada (June $15-19,1986)$.

B5. R. M. Summers, "Degraded Core Modeling in MELCOR," in "Proceedings of the Topical Meeting on Reactor Physics and Safety," Sandia National Laboratories report SAND86-1298C (NUREG/CP-0080) (September 1986), Vol. 2, p. 730.

B6. F. E. Haskin, S. W. Webb, and R. M. Summers, "Development and Status of MELCOR," in "Proceedings of the 14th Water Reactor Safety Research Information Meeting," Sandia National Laboratories report SAND86-2115C (NUREG/CP-0082) (October 1986).

B7. C. D. Leigh, R. K. Byers, and C. J. Shaffer, "MELCOR Validation Results," in "Proceedings of the 14th Water Reactor Safety Research Informatic " Meeting," Sandia National Laboratories repor SAND86-2128C (NUREG/CP-0082) (October 1986).

B8. S. E. Dingman, R. K. Cole. F. E. Haskin, R. M. Summers, and S. W. Webb, "Analysis of Peach Bottom Station Blackout with MELCOR," in "Proceedings of the 14 th Water Reactor Safety Research Information Meeting," Sandia National Laboratories repor SAND86-2129C (NUREG/CP-0082) (October 1986). 
B9. R. M. Summers, S. W. Webb, R. K. Cole, and S. E. Dingman, "MELCOR In-Vessel Modeling," in "Proceedings of the 15th Water Reactor Safety Research Information Meeting," Sandia National Laboratories report SAND87-1989C (NUREG/CP-0090) (October 1987).

B10. S. E. Dingman and A. C. Payne, Jr., "BWR Reactor Building Environments After Containment Failure," Transactions of the American Nuclear Society and the European Nuclear Society 1988 International Conference on Nuclear Fission: Fifty Years of Progress in Energy Security and Topical Meeting on TMI-2 Accident: Materials Behavior and Plant Recovery Technology, Vol. 57, pp. 357-359, Washington, District of Columbia (October 30-November 4, 1988).

B11. E. A. Boucheron and J. E. Kelly, "MELCOR Analysis of the TMI-2 Accident," in "Transactions of the American Nuclear Society and the European Nuclear Society 1988 International Conference on Nuclear Fission: Fifty Years of Progress in Energy Security and Topical Meeting on TMI-2 Accident: Materials Behavior and Plant Recovery Technology," Sandia National Laboratories report SAND88-1258C (November 1988), Vol. 57, p. 435-436.

B12. E. A. Boucheron and J. E. Kelly, "MELCOR Analysis of the Three Mile Island Unit 2 Accident," Nuclear Technology 87, p. 1050 (December 1989).

B13. R. M. Summers, J. C. Helton, and C. D. Leigh, "Incorporation of MELCOR Source Term Predictions Into Probabilistic Risk Assessments," in "Proceedings of the Intemational Topical Meeting on Probability, Reliability and Safety Assessment-PSA '89," Sandia National Laboratories report SAND88-1755C (April 1989), p. 366.

B14. S. E. Dingman and J. E. Kelly, "MELCOR Analyses of Drywell Flammability," Transactions of the American Nuclear Society 60 (1989).

B 15. G. D. Wyss, R. M. Summers, and L. A. Miller, "MELCOR Adaptation and Validation for Modeling of $\mathrm{N}$ Reactor Core Phenomena," in "Proceedings of the International Topical Meeting on the Safety, Status and Future of Non-commercial Reactors and Irradiation Facilities," Sandia National Laboratories report SAND90-0115C (October 1990).

B16. D. R. Bradley, Sandia National Laboratories, "Status of CORCON-MOD3 Development and Validation," Severe Accident Research Partners Meeting, draft preliminary report (May 2, 1990).

B 17. D. A. Powers, D. R. Bradley, E. Brockmann, and E. R. Copus, "Validation of Core Debris/Concrete Interactions and Source Term Models," presentation at International Seminar Fission Product Transport Processes in Reactor Accidents, Dubrovnik, Yugoslavia (May 22-26, 1989).

B18. R. L. Iman and J. C. Helton, "An Investigation of Uncertainty and Sensitivity Analysis Techniques for Computer Models," Risk Analysis 8 (1), 71-90 (1988). 
B 19. J. C. Helton, R. L. Iman, C. D. Leigh, and J. D. Johnson, "Uncertainty and Sensitivity Analysis of an Upper Plenum Test Problem for the MAEROS Aerosol Model," Aerosol Science and Technology 7, 199-216 (1987).

B20. J. C. Helton, R. L. Iman, J. D. Johnson, and C. D. Leigh, "Uncertainty and Sensitivity Analysis of a Dry Containment Test Problem for the MAEROS Aerosol Model," Nuclear Science and Engineering 102, 22-42 (1989).

B21. J. C. Helton, R. L. Iman, J. D. Johnson, and C. D. Leigh, "Uncertainty and Sensitivity Analysis of a Model for Multicomponent Aeroso! Dynamics," Nuclear Technology 73, 320-342 (June 1986).

\section{Reports and Papers External to SNL}

C1. W. T. Pratt, R. Denning, T. S. Kress, D. Powers, J. Gieseke, M. Khatib-Rahbar, and K. R. Perkins, "Evaluation of MELCOR as a Source Term Analysis Code," Brookhaven National Laboratory draft report (September 19, 1985).

C2. S. R. Greene, "The Impact of BWR MK I Primary Containment Failure Dynamics on Secondary Containment," presented at The 15th Water Reactor Safety Research Information Meeting, Gaithersburg, Maryland (NUREG/CP-OO90) (October 29, 1987).

C3. S. A. Ramsdale, "A Review of the Aerosol Models in the MELCOR 1.6.0 Code," United Kingdom Atomic Energy Authority, Safety and Reliability Directorate report (March 1988).

C4. I. R. Madni, "MELCOR Simulation of the PBF Severe Fuel Damage Test 1-1," in "Proceedings of the 26th National Heat Transfer Conference," AIChE Symposium Series on Heat Transfer, Philadelphia, Pennsylvania (1989).

C5 D. W. Golden, Ed., "TMI Analysis Exercise Final Report," OECD/CSNI Task Group on Three Mile Island draft report (to be published).

C6. M. I. Robertson, "MELCOR-A Modeling Overview and Calculations for Phase 1 of the BMC-F2 Experiment," United Kingdom Atomic Energy Authority, Safety and Reliability' Directorate report PWR/SAWG/P(90)578, PWR/FPERG/P(90)108 (May 1990).

C7. S. A. Hodge, "Boiling Water Reactor Severe Accident Models for MELCOR," Oak Ridge National Laboratory letter report ORNL/NRC/LTR-90/13 to US Nuclear Regulatory Commission (May 22, 1990).

C8. "The Response of BWR Mark-III Containments to Short-Term Station Blackout Severe Accident Sequences," Oak Ridge National Laboratory report ORNL/NRC/LTR-89/13, Sections 2.2.3.2, Appendix A, and Appendix D (December 29, 1989).

C9. C. R. Hyman, "Current Limitations in the Application of "CONTAIN/ CORCON to BWR Mark-I Severe Accident Analyses, Oak Ridge National Laboratory document (January 27, 1989).

C10. T. S. Kress, Compiler, "Review of the Status of Validation of the Computer Codes Used in the Severe Accident Source Term Reassessment Study (BMI-2104)," Oak Ridge National Laboratory document ORNL/TM-8842. (April 1985). 
C11. J. Valente and D. Mirkovic, "MAAP 3.0B," Safety and Risk Evaluation Division, Department of Nuclear Energy, Brookhaven National Laboratory draft document (May 1990).

C12. J. W. Yang and D. Mirkovic, "MAAP 3.0B Review/PWR Review, Part 1: RCS Thermal Hydraulics and Heat Transfer, Application for Success Criteria," Safety and Risk Evaluation Division, Department of Nuclear Energy, Brookhaven National Laboratory draft document (May 1990).

C13. J. W. Yang, "MAAP 3.0B Review/PWR Review, Part 2: In-Vessel Melt Progression," Safety and Risk Evaluation Division, Department of Nuclear Energy, Brookhaven National Laboratory draft document (August 1990).

C14. I. K. Madni, "MELCOR Simulation of Long-Term Station Blackout at Peach Bottom," in "Proceedings of the 18th Water Reactor Safety Information Meeting," Brookhaven National Laboratory document NUREG/CP-0113 (October 1990).

C15. I. K. Madni, "MELCOR Modeling of the PBF Severe Fuel Damage Test 1-4," Brookhaven National Laboratory document BNL-NUREG-44503 (1990).

C16. M. I. Robertson, "Validation of Control Volume Thermal-Hydraulic Modeling," presentation at CSNI/CEC Workshop on Aerosol Behaviour in the Containment, Fontenay-aux-Roses, France (November 1990).

C17. I. K. Madni, "MELCOR 'Lessons Learned': A User Perspective," Brookhaven National Laboratory draft letter report to R. Foulds, US Nuclear Regulatory Commission (January 9, 1991).

C18. T. Haste, "Summary of AEA Technology, SRD, Views on the Use of the MELCOR Code," AEA Technology, Winfrith Technology Center, United Kingdom letter to attention of MELCOR Peer Review Committee (Lecember 19, 1990).

C19. J. U. Valente, "MAAP 3.0B/BWR Review, Technical Evaluation Report 3: Severe Accident Containment Modeling," Brookhaven National Laboratory report, Safety and Risk Evaluation Division, Department of Nuclear Energy (October 1990).

C20. J. W. Yang, "MAAP 3.0B Review/PWR Review, Part 4: Fission Product Release and Transport," Brookhaven National Laboratory draft document, Safety and Risk Evaluation Division, Deparment of Nuclear Energy (December 1990).

C21. S. A. Hodge, "Debris Bed Behavior in the BWR Lower Plenum-The BWRSAR Approach with Recommended Improvements for Installation in MELCOR," Oak Ridge National Laboratory document ORNL/NRC/LTR-90/10 (April 26, 1990).

C22. I. K. Madni and X. D. Guo, "MELCOR Simulation of the Full-Length HighTemperature 2 Experiment," Brookhaven National Laboratory Safety Integration Group document NRC FIN A-3281 (1991).

C23. S. L. Chan, H. P. Isaak, U. Schmocker, and M. Khatib-Rahbar, "Characterization of Severe Accidents Using MELCOR: A Perspective," Swiss Federal Nuclear Safety Inspectorate, presentation at the Cooperative Severe Accident Research Program SemiAnnual Review Meeting, Bethesda, Maryland (May 6-10, 1991). 
C24. E. L. Fuller and J: A. Maly, "Progress in MAAP Development and Use as Illustrated by Station Blackout Accident Studies," Electric Power Research Institute, presentation at the Cooperative Severe Accident Research Program Semi-Annual Review Meeting, Bethesda, Maryland (May 6-10, 1991).

\section{Correspondence and Memoranda}

D1. R. O. Meyer, "MELCOR Meeting with Source Term Experts," US Nuclear Regulatory Commission memorandum to M. Silberberg, US Nuclear Regulatory Commission (June 14, 1985).

D2. J. E. Kelly, letter to M. A. Cunningham, US Nuclear Regulatory Commission, comparing MELCOR and MAAP (January 18, 1988).

D3. J. E. Kelly, letter to P. K. Niyogi, US Nuclear Regulatory Commission, comparing MELCOR and HECTR (November 18, 1988).

D4. J. E. Kelly, letter to P. K. Niyogi, US Nuclear Regulatory Commission, comparing MELCOR and STCP (March 9, 1989).

D5. B. W. Sheron, "Retirement of the Source Term Code Package and the Use of MELCOR for Severe Accident Plant Analyses," US Nuclear Regulatory Commission memorandum for E. S. Beckjord (July 11, 1989).

D6. W. Beckner, "Need for Continuing MELCOR Support for Mark-II CPI Program," US Nuclear Regulatory Commission memorandum for F. Costanzi (August 15, 1989).

D7. F. A. Costanzi, "Need for Continued MELCOR Support," US Nuclear Regulatory Commission memorandum for W. Beckner (September 27, 1989).

D8. G. M. Martinez, "MELCOR Post-Test Calculations of the HDR Experiment," letter repont to R. B. Foulds US Nuclear Regulatory Commission (September 29, 1989).

D9. R. M. Summers, "Comparison of MELCOR \& BWRSAR In-Vessel Modeling Approach," Sandia National Laboratories unpublished data (September 15, 1989).

D10. J. E. Kelly, "ORNL Suggestions for MELCOR Improvements," Sandia National Laboratories letter to F. Eltawila, US Nuclear Regulatory Commission (October 4, 1989).

D11. J. E. Kelly, "Recommendations by Steve Hodge, ORNL, for BWR In-Vessel Modeling Improvements in MELCOR," Sandia National Laboratories letter to F. Eltawila, US Nuclear Regulatory Commission (December 21, 1989).

D12. K. E. Washington and R. M. Summers, "CONTAIN and MELCOR: A Comparison of Models, Features, and Scope," transmitted by letter from J. E. Kelly and K. D. Bergeron to F. Eltawila, US Nuclear Regulatory Commission (November 21, 1989).

D13. R. M. Summers, "MELCOR Modeling Caveats," Sandia National Laboratories draft document (January 10, 1990). 
D14. R. M. Summers, "MELCOR 1.8.0 Shortcomings," Sandia National Laboratories draft document (January 10, 1990).

D15. C. Ryder, "Problems in MELCOR," informal memorandum to J. Glynn (January 31, 1990).

D16. R. M. Sumrers, K. K. Cole, Jr., and E. A. Boucheron, "MELCOR Improvement Needs Assmernottt," Sandia National Laboratories letter report to R. B. Foulds, US Nuclear Regulatriry Commission (October 17, 1990).

D17. S. R. Greene, "Transmittal of ORNL Documentation to MELCOR Code Assessment Team," Oak Ridge National Laboratory letter to Brent Boyack, Los Alamos National Laboratory (June 28, 1990).

D18. R. Meyer, "MELCOR Meeting at Sandia," US Nuclear Regulatory Commission memorandum to M. Silberberg (March 7, 1985) (contains statement of original code objectives).

D19. E. A. Boucheron, "TMI-2 Assessment Efforts," Sandia National L boratories letter to R. B. Foulds, US Nuclear Regulatory Commission (September 21, 1989) (supplements Refs. B 11 and B 12 by including an extensive discussion of modeling needs).

D20. R. M. Summers, "Transmittal of MELCOR Roadmaps," Sandia National Laboratories letter to B. E. Boyack (August 14, 1990). List of roadmaps follows:

R. K. Cole, Jr., Roadmap to CVH/FL Package Documentation,

M. K. Carmel, Roadmap for References to Heat Structure Package Modeling,

R. M. Summers, Roadmap to Core Modeling in MELCOR Documentation,

E. A. Boucheron, Roadmap to Fission Product Modeling in MELCOR Documentation,

R. M. Summers, Roadmap to Fuel Dispersal Interactions Modeling in MELCOR Documentation,

R. K. Cole, Jr., Roadmap to CAV Package Documentation,

R. M. Summers, Roadmap to Gas Combustion Modeling in MELCOR Documentation,

R. M. Summers, Roadmap to Engineered Safety Features Modeling in MELCOR Documentation,

R. K. Cole, Jr., Roadmap to CVT Package Documentation,

R. M. Summers, Roadmap to Material Properties Modeling in MELCOR Documentation, and

Roadmap to Decay Heat Modeling in MELCOR Documentation.

D21. D. R. Bradley, R. K. Cole, and A. W. Shiver, "First Correction Set for CORCONMOD2, Sandia National Laboratories memorandum (July 11, 1986).

D22 D. R. Bradley, R. K. Cole, and A. W. Shiver, "Second Correction Set for CORCON-MOD2, Sandia National Laboratories memi randum (August 15, 1986).

D23. D. R. Bradley, R. K. Cole, and A. W. Shiver, "Third Correction Set for CORCONMOD2, Sandia National Laboratories memorandum (March 17, 1987).

D24. D. R. Bradley and A. W. Shiver, "Fourth Correction Set for CORCON-MOD2, Sandia National Laboratories memorandum (June 26, 1987).

D25. MELCOR Defect Investigation Report 670, MELCOR Update 1.8CK (January 1, 1990). 
D26. MELCOR Defect Investigation Report 702, 703, MELCOR Update 1.8CW (May 3, 1990).

D27. MELCOR Defect Investigation Report 703, MELCOR Update 1.8DB (May 24, 1990).

D28. MELCOR Defect Investigation Report 299 (July 27, 1987).

D29. MELCOR Defect Investigation Report ORNL-57 (April 27, 1989).

D30. F. Eric Haskin, "Whole-Core Decay Heat Power," Sandia National Laboratories memorandum (January 28, 1986).

D31. S. Dingman, "MELCOR Blind Calculations for HDR Tests 31.4 and 31.5," Sandia National Laboratories, Thermal/Hydraulic Analysis Division document (undated).

D32. E. A. Boucheron, "MELCOR Quality Control and Technical Assessment, Preliminary Assessment Recommendations and Data Compilation," Sandia National Laboratories, Thermal/Hydraulic Division draft document (Spring 1990).

D33. F. Eltawila, Accident Evaluation Branch, Division of Systems Research, Office of Nuclear Regulatory Research letter to B. E. Boyack, Los Alamos National Laboratory (November 2, 1990).

D34. B. W. Sheron, "NRC/RES Software Docurnentation Guidance," US Nuclear Regulatory Commission, Division of Systems Research letter to T. Hirons, Los Alamos National Laboratory (April 18, 1991).

D35. R. M. Summers, "Updates to MELCOR 1.8.0 to Create Version 1.8.1," Sandia National Laboratories draft letter to R. B. Foulds, US Nuclear Regulatory Commission (May 1, 1991).

Qther

E1. J. E. Kelly, "MELCOR User's Group Bi-Monthly Newsletter for the months of April, May, June, and July 1989," Sandia National Laboratories letter to R. B. Foulds, US Nuclear Regulatury Conimission (August 25, 1989).

E2. MELCOR Users Newsletter 5 (1), Sandia National Laboratories (May 1990).

E3. "MAAP Design Review," Jason Associates draft report (October 1989).

E4. MELCOR Users Newsletter 5 (2), Sandia National Laboratories (August 1990).

\section{Committee Documents and Findings}

F1. J. S. Elson, "MELCOR Peer Review: MELCOR Decay Heat Package," Los Alamos National Laboratory Group N-12 memorandum N-12-90-609 to B. E. Boyack (October $16,1990)$.

F2. R. K. Cole, "Response to Professor Dhir's Comments on MELCOR CAV Package," Sandia National Laboratories letter to B. Boyack (October 30, 1990).

B- 9 
F3. J. S. Elson, "MELCOR Decay Heat Package-Addendum," Los Alamos National Laboratory Group N-12 memorandum N-12-90-721 to B. E. Boyack (November 28, 1990).

F4. B. E. Boyack, Los Alamos National Laboratory, unpublished questions on "Heat Structure Thermal Response," by V. Dhir (November 8,1990 ), unpublished answers by M. K. Carmel, Sandia National Laboratories, Second MELCOR Peer Review Meeting, November 5-7, 1990.

F5. B. E. Boyack, Los Alamos National Laboratory, unpublished questions on "Core Concrete Interactions," by V. Dhir (November 12, 1990), unpublished answers by R. K. Cole, Sandia National Laboratories, Second MELCOR Peer Review Meeting, November 5-7, 1990.

F6. B. E. Boyack, Los Alamos National Laboratory, unpublished questions on "Heat Structures (HS) Package" and "Core Heatup and Degradation (COR) Package," by R. Viskanta, in letter to B. E. Boyack, Regarding: Questions Concerning MELCOR to be addressed by SNL at the November 5-7, 1990 Committee Meeting, September 24, 1990. Unpublished answers on "Heat Structure (HS) Package" by M. K. Carmel, Sandia National Laboratories, Second MELCOR Peer Review Meeting, November 5--7, 1990. Unpublished answers on "Core Heatup and Degradation (COR) Package" by R. M. Summers, Sandia National Laboratories, Second MELCOR Peer Review Meeting, November 5-7, 1990.

F7. B. E. Boyack, Los Alamos National Laboratory, unpublished questions on "MELCOR Hydrodynamics" and "Core-Concrete Models," by M. Khatib-Rahbar, in letter to B. E. Boyack, Regarding: Preliminary Questions on MELCOR Hydrodynamics and CoreConcrete Models, October 2, 1990. Unpublished answers on "Responses to Dr. KhatibRahbar's comments on MELCOR CVH/FL Package," by R. K. Cole, Sandia National Laboratories, Second MELCOR Peer Review Meeting, November 5-7, 1990. Unpublished answers on "Responses to Dr. Khatib-Rahbar's comments on MELCOR CAV package," by R. K. Cole, Sandia National Laboratories, Second MELCOR Peer Review Meeting, November 5-7, 1990.

F8. B. E. Boyack, Los Alamos National Laboratory, unpublished questions on "Core Modeling" and "Material Properties," by T. J. Haste, in letter to B. E. Boyack, Regarding: MELCOR Peer Review-Second Meeting, September 27, 1990. Unpublished answers on "Answers to Tim Haste's Questions on COR Package Modeling" by R. M. Summers, Sandia National Laboratories, Second MELCOR Peer Review Meeting, November 5-7, 1990. Unpublished answers on "Responses to Additional Haste Questions on COR Package Modeling," by R. M. Summers, Third MELCOR Peer Review Meeting, January 11, 1991.

F9. B. E. Boyack, Los Alamos National Laboratory, unpublished questions on "BUR Package," by M. Kenton, Second MELCOR Peer Review Meeting, November 5-7, 1990. Unpublished answers on "Responses to Kenton Questions on BUR Package Modeling," by R. M. Summers and S. Dingman, Sandia National Laboratories, Third MELCOR Peer Review Meeting, January 14-16, 1991.

F10. B. E. Boyack, Los Alamos National Laboratory, unpublished questions on "SPR and FCL Packages," by M. Kenton, Second MELCOR Peer Review Meeting, November 57, 1990. Unpublished answers on "Responses to Kenton Questions on SPR and FCL 
Package Modeling" by R. M. Summers, Sandia National Laboratories, Third MELCOR Peer Review Meeting, January 14-16, 1991.

F11. B. E. Boyack, Los Alamos National Laboratory, unpublished questions on "HS and COR Packages," by R. Viskanta, in letter to B. E. Boyack (December 27, 1990). Unpublished answer on "Responses to Additional Viskanta Questions on HS Package Modeling" by R. M. Summers, Sandia National Laboratories, Third MELCOR Peer Review Meeting, January 11, 1991. Unpublished answer on "Responses to Additional Viskanta Questions on COR Package Modeling," by R. M. Summers, Sandia National Laboratories, Third MELCOR Peer Review Meeting, January 11, 1991.

F12. B. E. Boyack, Los Alamos National Laboratory, unpublished questions on "RN Package," by R. S. Denning, in letter to B. E. Boyack (October 16, 1990). Unpublished answers on "Issues to be Addressed for RN Package," by E. Boucheron, Sandia National Laboratories, Third MELCOR Peer Review Meeting, January 14-16, 1991.

F13. T. Haste, "Summary of Discussions on Decay Heat and Material Properties on November 8, 1990," Thermal Reactor Services, AEA Technology, Winfrith Technology Center summary report (December 18, 1990).

F14. B. E. Boyack and J. Richardson, "Final Minutes of the First MELCOR Peer Review Meeting," Los Alamos National Laboratory Group N-12 letter N-12-90-518 (September $5,1990)$.

F15. B. E. Boyack and J. Richardson, "Final Minutes of the Second MELCOR Peer Review Meeting," Los Alamos National Laboratory Group N-12 letter N-12-91-54 (January 24, 1991).

F16. B. E. Boyack and J. Richardson, "Final Minutes of the Third MELCOR Peer Review Meeting," Los Alamos National Laboratory Group N-12 letter N-12-91-171 (March 20, 1991).

F17. B. E. Boyack and J. Richardson, "Final Minutes of the Fourth MELCOR Peer Review Meeting," Los Alamos National Laboratory Group N-12 letter N-12-91-382 (June 4, 1991).

F18. B. E. Boyack and J. Richardson, "Final Minutes of the Fifth MELCOR Peer Review Meeting," Los Alamos National Laboratory Group N-12 letter N-12-91-480 (July 15, 1991).

F19. B. E. Boyack, "Actions From 5th MELCOR Peer Review Meeting," Los Alamos National Laboratory Group N-12 letter N-12-91-378 (June 3, 1991).

F20. M. Kenton, "Transmittal of Viewgraphs on MAAP/MELCOR Comparison Calculations," Gabor, Kenton and Associates, Inc., letter to B. Boyack (April 9, 1991). 


\section{APPENDIX C}

\section{Severe Accident Phenomena}

by

\section{Khatib-Rahbar}

This appendix provides a list of dominant physical phenomena against which the existence, adequacy, and, when possible, fidelity of each MELCOR model will be assessed.

On a generic basis, the various "top-level" physical phenomena contributing to each phase of severe accident progression are delineated for both BWKs and PWRs. It is recognized that the importance of individual phenomenon will vary depending on the specific accident sequence under consideration and the intended application. including:

MELCOR is expected to be applicable to a wide spectrum of severe accident conditions,

(1) high-and low- (with respect to RCS) pressure sequences,

(2) scenarios leading to early (ECCS fails early) and late (ECCS fails late) initiation of core degradation,

(3) sequences involving operation and failure of containment heat removal systems,

(4) sequences leading to early and late containment failures through a variety of failure modes, and

(5) recoverable accidents.

MELCOR should provide reasonable estimates of severe accident behavior under these diverse accident conditions. To the extent possible, the code should include reasonable mechanistic estimates of severe accident phenomena, including sufficient coverage of the dominant severe accident physics and chemistry within an integrated, fast-runniug, easy to use parametric framework. However, MELCOR is not envisioned to become the test bed for resolution of phenomenological uncertainty issues.

Typically, severe accident analyses are performed to help in understanding the behavior of plant and containment systems during postulated accident conditions. These studies are often conducted in support of PRAs, or as added information for regulatory decision-making (i.e., evaluation of potential severe accident management strategies). As part of these studies, computer codes are exercised to evaluate key accident signatures including some of the following:

(1) timing of key events (core uncovery, lower plenum dryout, vessel breach, containment failure, etc.),

(2) important fission product attributes (release from fuel, retention within RCS, retention in pools, etc.), 
(3) temperatures of RCS structures (lower head, hot leg, steam generator tubes, etc),

(4) RCS pressure prior to vessel breach,

(5) mode and location of RCS failure (bottom head, hot leg, steam generator tubes, etc.),

(6) quantity and rate of hydrogen generation (in-vessel and ex-vessel),

(7) core debris quantity, composition, temperature, and rate of ejection into containment,

(8) downward and lateral ablation of concrete basemat,

(9) debris configuration, location, and state on concrete basemat,

(10) interaction of molten debris with water,

(11) rate, quantity, and composition of noncondensable gas generation from coreconcrete interactions,

(12) temperatures and pressures of various containment compartments,

(13) atmospheric composition of various containment compartments,

(14) temperature and pressure loads resulting from hydrogen combustion,

(15) impact of containment Engineered Safety Features (fans, sprays, pools), and

(1) quantity, composition, time, duration, elevation, energy, and rate of release of fission products to environment (accident source term).

The decomposition proposed here is based on the premise that a complete, integrated analysis must portray important phenomenological processes during the various phases of accidents. Specifically, both the dominant in-vessel and ex-vessel processes must be included.

For the in-vessel phase, four distinct interval, are considered, namely:

(1) initial transient, depletion, and heatup interval (before core damage),

(2) core uncovery interval (start of core damage),

(3) melt relocation and slump interval (substantial damage), and

(4) core debris material inside the lower plenum interval (late in-vessel phase).

The ex-vessel phase of accidents includes those processes resulting from interaction of core/debris with the concrete containment floor, as well as processes that may originate in-vessel, but become dominant following reactor pressure vessel failure. This includes revaporization of previously deposited fission products from RCS surfaces.

For each interval, key phenomenological issues impacting the evolution of the accident sequence are delineated. For the process to remain trictable, detailed subissucs resulting from higher order phenomena associated with interaction of various physical and chemical prosesses are intentionally not shown. This should not mean that the dominance of some of these phenomena is 
to be ignored as part of MELCOR assessment. It is assumed that the individual committee members cognizant of various phenomenological modules will recognize these and address them as part of their review.

Flow charts for the severe accident phenomena follow. 


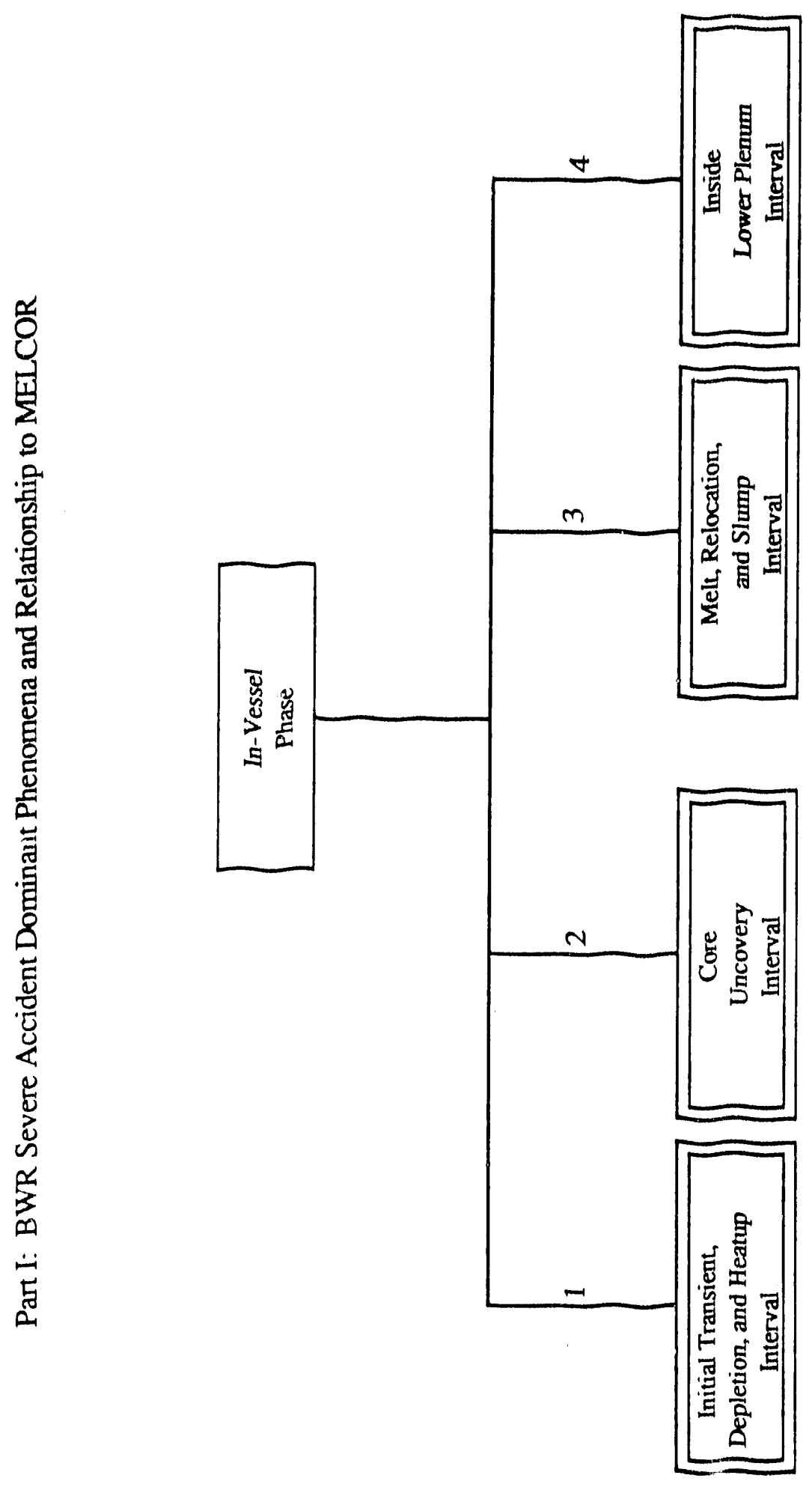

C- 4 


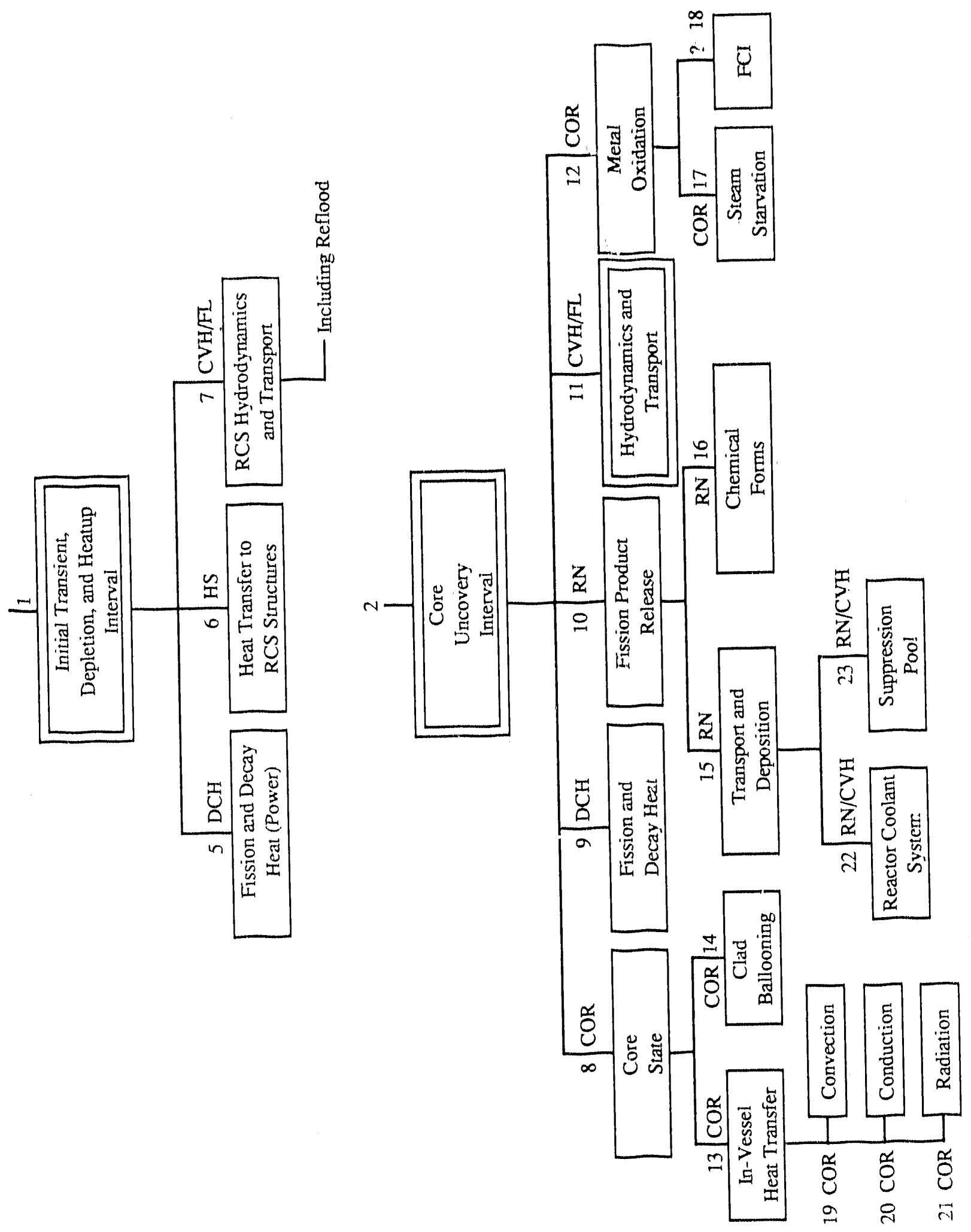




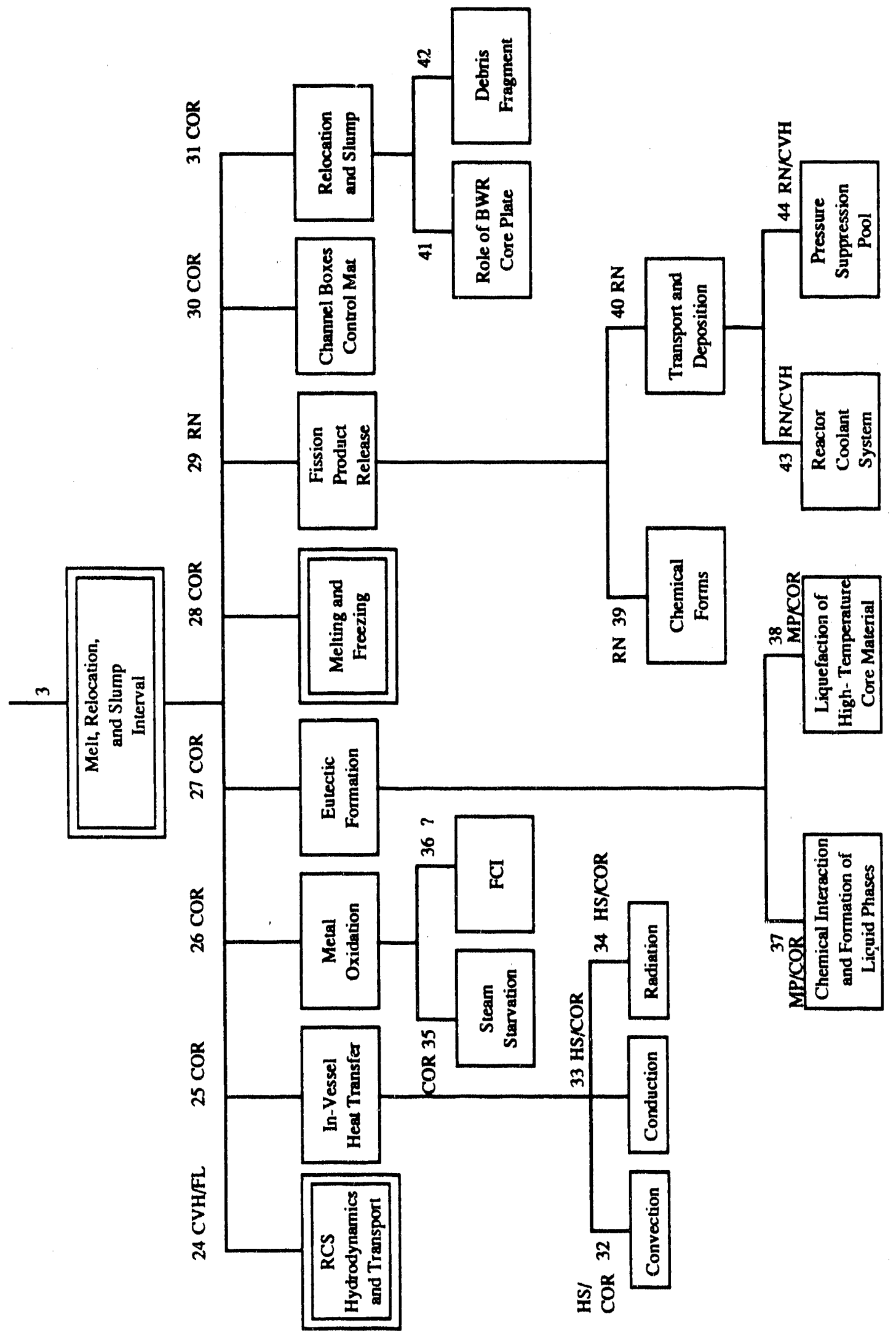

C- 6 


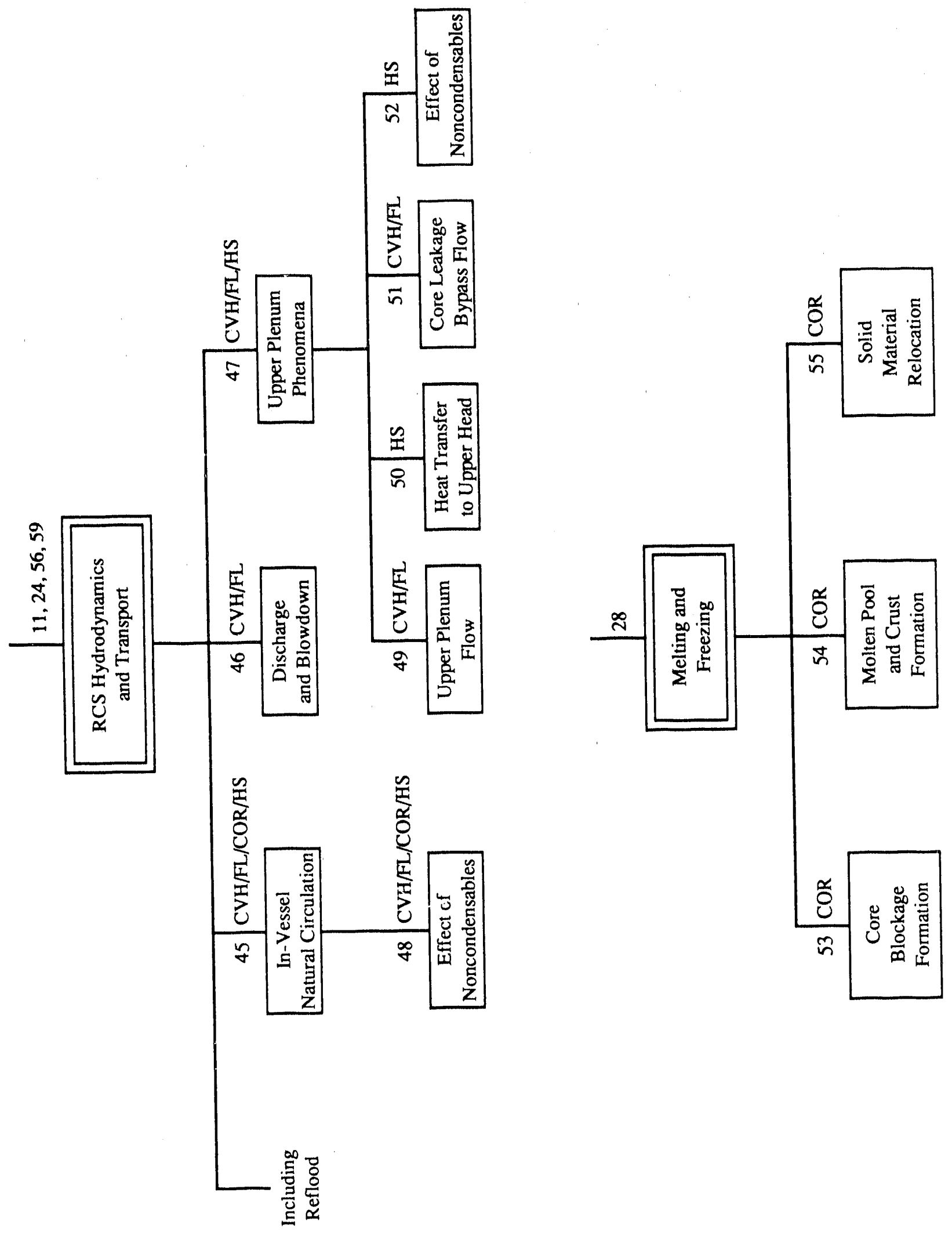




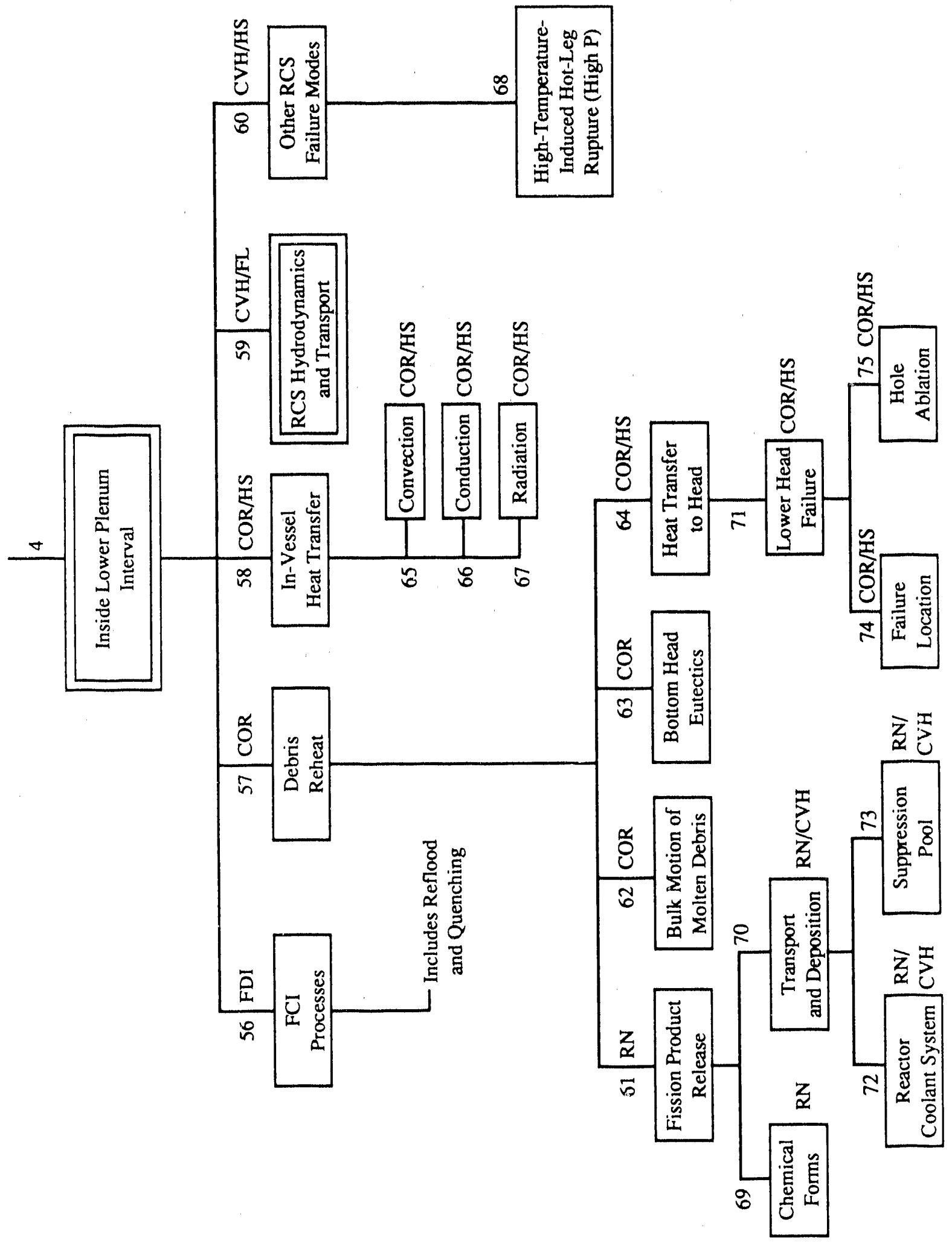




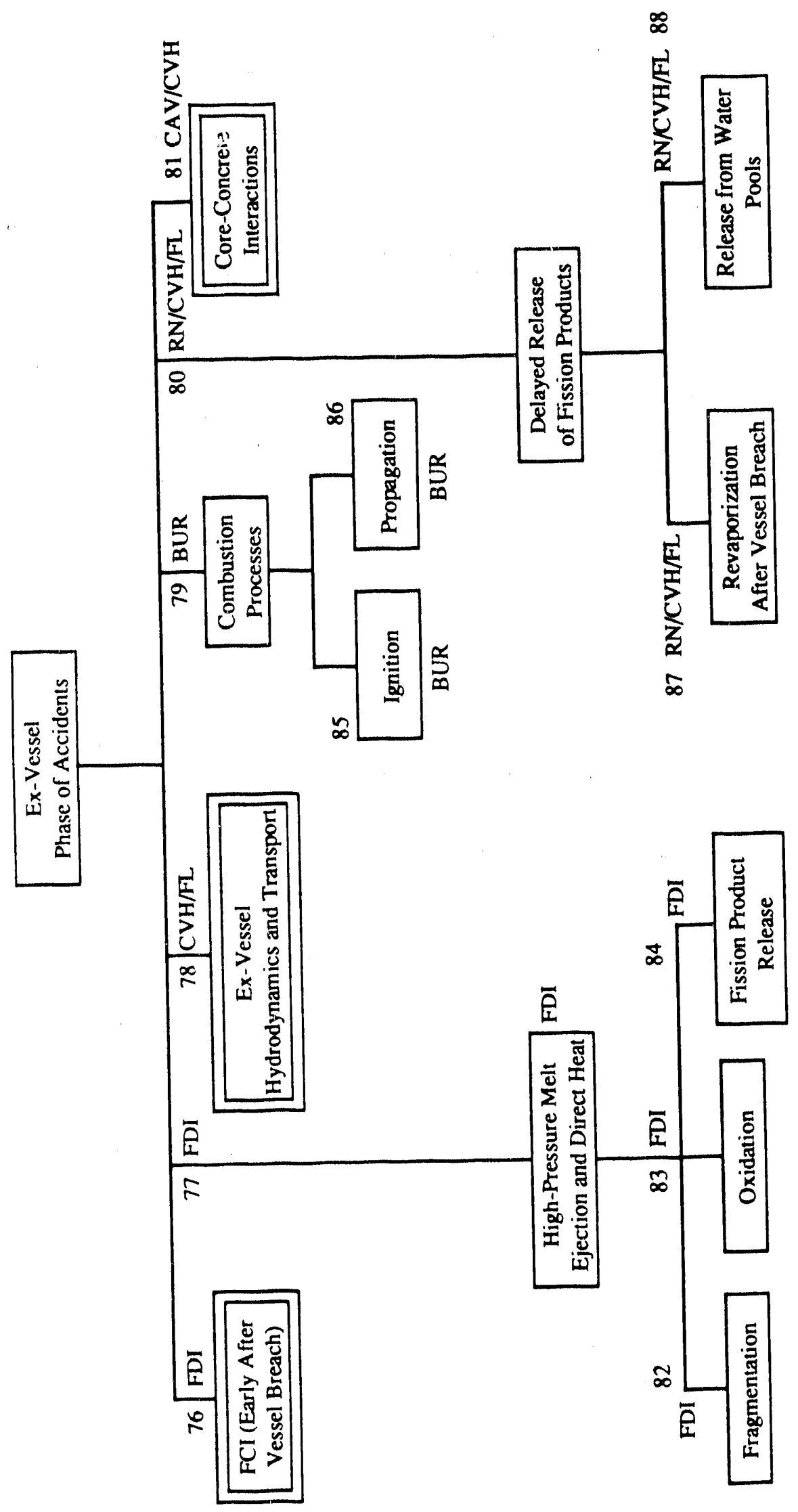




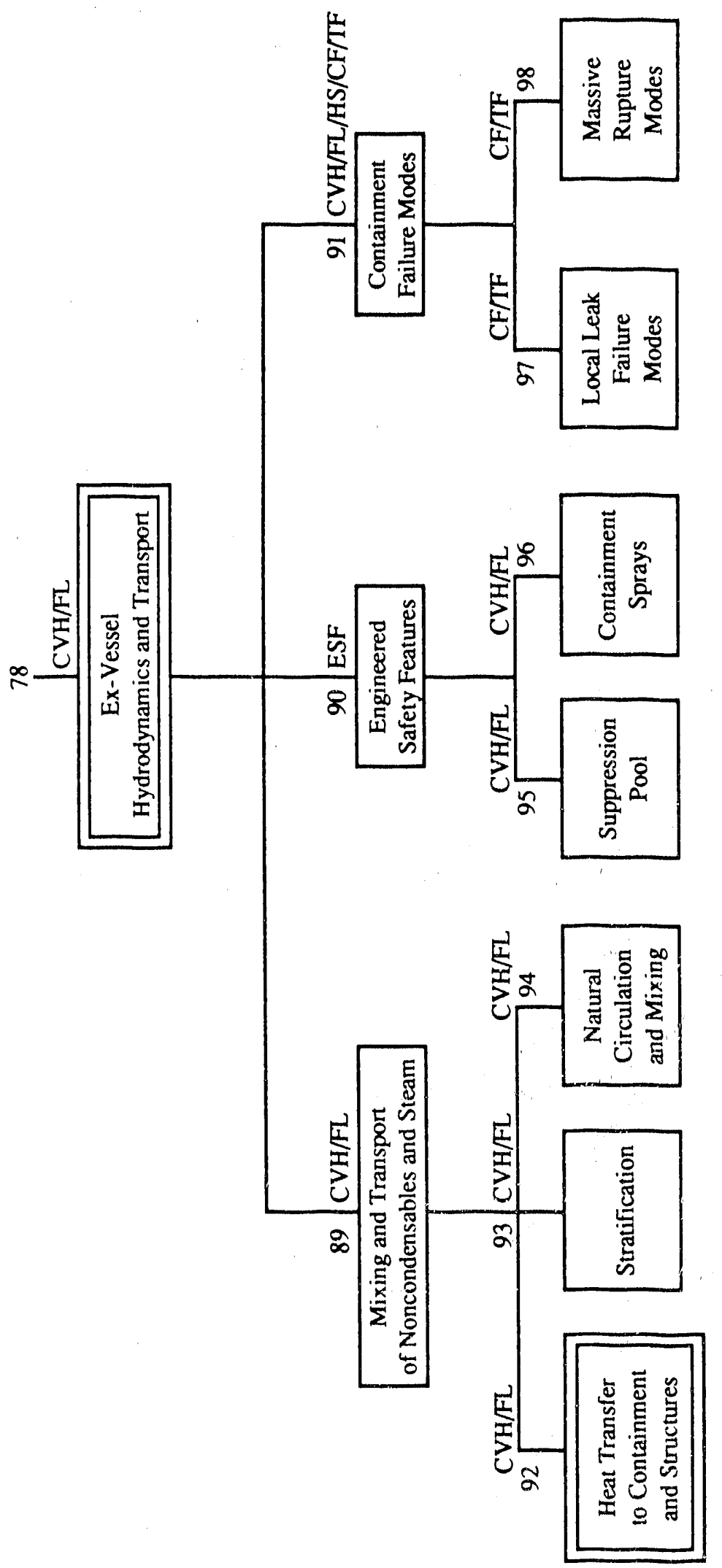

C- 10 


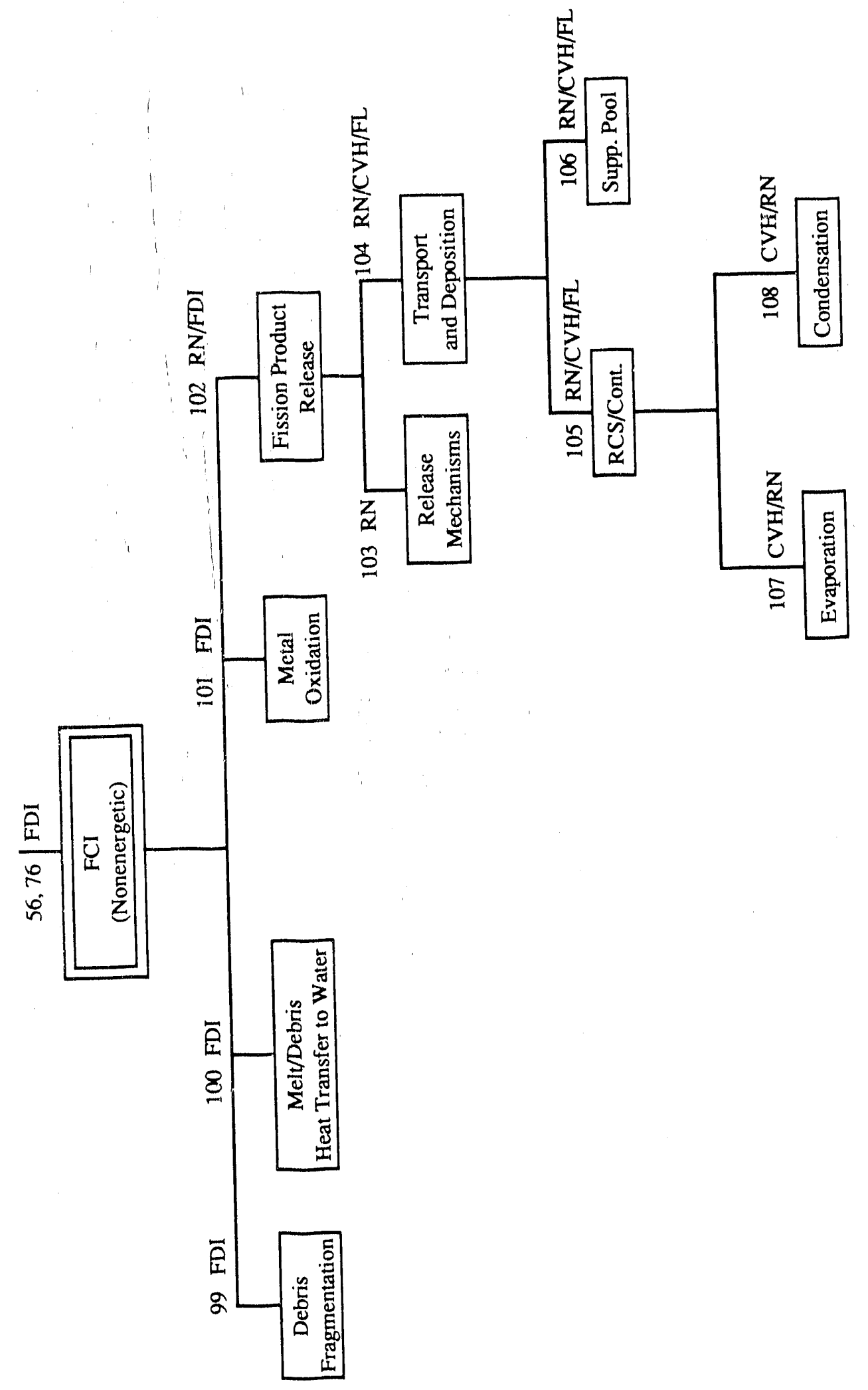

C-11 


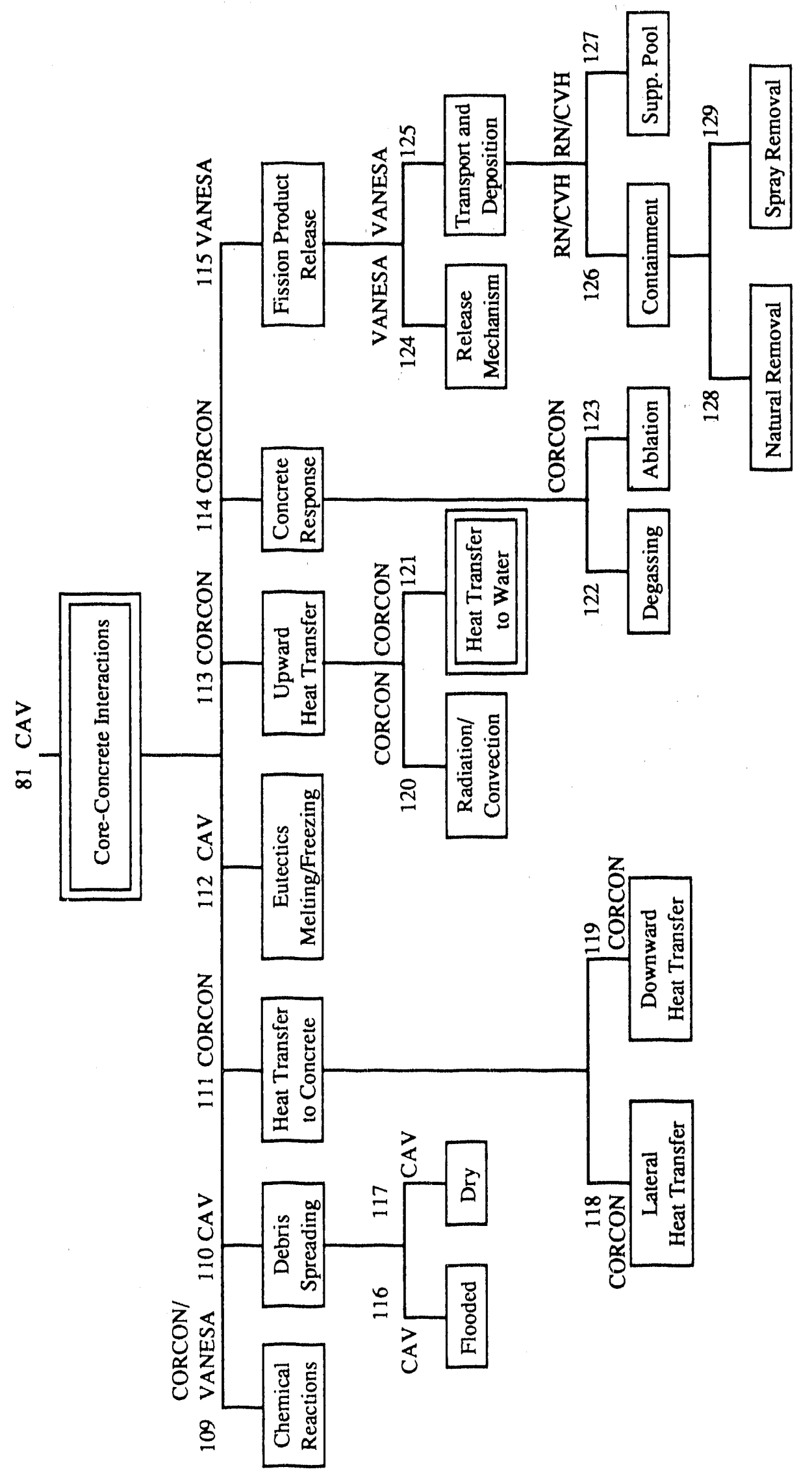

C- 12 

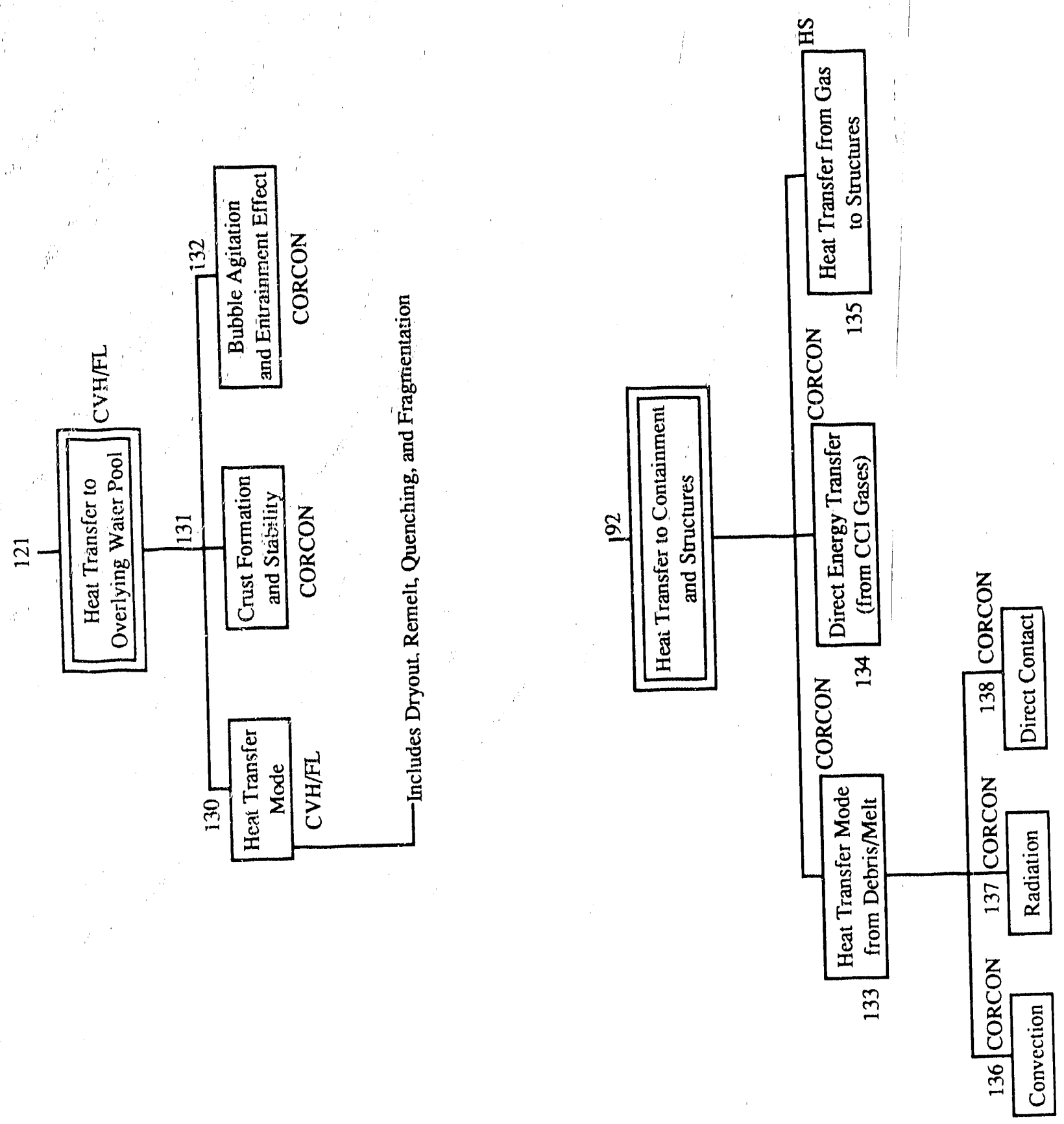


\section{In-Vessel Phase of Accidents}

1. Initial Transient, Depletion and Heatup Interval

5. Fission and Decay Heat (Power)

$\mathrm{DCH}$

6. Heat Transfer to RCS Structures

HS

7. RCS Hydrodynamics and Transport

CVH, FL

2. Core Uncovery Interval

8. Core State

13. In-Vessel Heat Transfer

COR

19. Convection

20. Conduction

21. Radiation

14. Clad Ballooning

9. Fission and Decay Heat

$\mathrm{DCH}$

10. Fission Product Release

15. Transport and Deposition

22. Reactor Coolant System

23. Pressure Suppression Pool

16. Chemical Forms

11. Hydrodynamics and Transport ${ }^{\mathrm{a}}$

45. In-Vessel Natural Circulation

48. Effects of Noncondensables

46. Discharge and Blowdown

47. Upper Plenum Phenomena

49. Upper Plenum Flow

50. Heat Transfer to Upper Head and Internals

$\mathrm{RN}, \mathrm{COR}$

51. Core Bypass Leakage Flow

52. Effects of Noncondensables

12. Metal Oxidation

17. Steam Starvation

18. Fuel Coolant Interactions (FCI)

$\mathrm{CVH}, \mathrm{FL}$

CVH/FL/COR/HS

$\mathrm{CVH} / \mathrm{FL} / \mathrm{COR} / \mathrm{HS}$

$\mathrm{CVH} / \mathrm{FL}$

$\mathrm{CVH} / \mathrm{FL}$

CVH/HS

CVH/FL/COR

$\mathrm{CVH} / \mathrm{HS}$

COR

$\mathrm{COR} / \mathrm{CVH} / \mathrm{FL}$

COR

3. Melt, Relocation, and Slump Interval

24. RCS Hydrodynamics and Transpurt ${ }^{\mathrm{a}}$

CVH/FL

45. In-Vessel Natural circulation

48. Effects of Noncondensables

46. Discharge and Blowdown

47. Upper Plenum Phenomena

49. Upper Plenum Flow

50. Heat Transfer to Upper Head and Internals

51. Core Bypass Leakage Flow

52. Effects of Noncondensables

25. In-Vessel Heat Transfer

32. Convection

$\mathrm{CVH} / \mathrm{FL} / \mathrm{COR} / \mathrm{HS}$

CVH/FL/COR/HS

CVH/FL

CVH/FL

$\mathrm{CVH} / \mathrm{HS}$

CVH/FL/COR

CVH/HS

HS/COR 
33. Conduction

34. Radiation

26. Metal Oxidation

35. Steam Starvation

36. Fuel Coolant Interactions (FCI)

27. Eutectic Formation

37. Chemical Interactions and Formation of Liquid Phases

38. Liquefaction of High-Temperature Core Material

28. Melting and Freezing

53. Core Debris Blockage Formation

54. Molten Pool and Crust Formation

55. Solid Material Relocation

29. Fission Product Release

39. Chemical Forms

40. Transport and Deposition

43. Reactor Coolant System

44. Pressure Suppression System

30. Channel Boxes and Control Material

31. Relocation and Slump

41. Role of BWR Core Plate

42. Debris Fragmentation

$\mathrm{HS} / \mathrm{COR}$

$\mathrm{HS} / \mathrm{COR}$

COR

$\mathrm{COR} / \mathrm{CVH} / \mathrm{FL}$

COR

COR

$\mathrm{MP} / \mathrm{COR}$

$\mathrm{MP} / \mathrm{COR}$

COR

COR

COR

COR

RN

RN

RN

$\mathrm{RN} / \mathrm{CVH}$

$\mathrm{RN} / \mathrm{CVH}$

$\mathrm{COR} / \mathrm{MP}$

COR

$\therefore$ OR

COR

4. Inside Lower Plenum Interval

56. FCI Processes

99. Debris Fragmentation

100. Melt/Debris Heat Transfer to Water

101. Metal Oxidation

102. Fission Product Release

103. Release Mechanisms

104. Transport and Deposition

105. Reactor Coolant System/Containment

107. Evaporation

108. Condensation

106. Pressure Suppression System

57. Debris Reheat

61. Fission Product Release and Transport

69. Chemical Forms

70. Transport and Deposition

72. Reactor Coolant System

73. Pressure Suppression System

62. Bulk Motion of Molten Debris

63. Bottom Head Eutectics

64. Heat Transfer to Lower Head

71. Lower Head Failure

74. Failure Location

FDI

FDI

FDI

FDI

RN

RN

$\mathrm{RN}$

$\mathrm{RN} / \mathrm{CVH}$

$\mathrm{CVH} / \mathrm{RN}$

CVH/RN

$\mathrm{RN} / \mathrm{CVH}$

COR

RN

RN

RiN

$\mathrm{RN} / \mathrm{CVH}$

$\mathrm{RN} / \mathrm{CVH}$

COR

COR/MP

COR/HS

COR/HS/TF/CF

COR/HS

75. Hole Ablation

$\mathrm{COR} / \mathrm{HS}$ 
58. In-Vessel Heat Transfer

65. Convection

66. Conduction

67. Radiation

59. RCS Hydrodynamics and Transport

45. In-Vessel Natural Circulation

48. Effects of Noncondensables

46. Discharge and Blowdown

47. Upper Plenum Phenomena

49. Upper Plenum Flow

50. Heat Transfer to Upper Head and Internals

51. Core Bypass Leakage Flow

52. Effects of Nonconsensables

60. Other RCS Failure Modes

68. High-Temperature-Induced Hot-Leg Failure (High P)

COR/HS

$\mathrm{HS} / \mathrm{COR}$

$\mathrm{HS} / \mathrm{COR}$

$\mathrm{HS} / \mathrm{COR}$

$\mathrm{CVH} / \mathrm{FL}$

$\mathrm{CVH} / \mathrm{FL} / \mathrm{COR} / \mathrm{HS}$

$\mathrm{CVH} / \mathrm{FL} / \mathrm{COR} / \mathrm{HS}$

$\mathrm{CVH} / \mathrm{FL}$

$\mathrm{CVH} / \mathrm{FL}$

$\mathrm{CVH} / \mathrm{HS}$

$\mathrm{CVH} / \mathrm{FL} / \mathrm{COR}$

$\mathrm{CVH} / \mathrm{HS}$

$\mathrm{CVH} / \mathrm{HS}$

$\mathrm{CVH} / \mathrm{HS} / \mathrm{TF} / \mathrm{CF}$

\section{Ex-Vessel Phase of Accidents}

76. FCI (Early After Vessel Breach) FDI

99. Debris Fragmentation

FDI

100. Melt/Debris Heat Transfer to Water FDI

101. Metal Oxidation

FDI

102. Fission Product Release

103. Release Mechanisms

RN

104. Transport and Deposition

RN

105. Reactor Coolant System/Containment

RN

107. Evaporation

$\mathrm{RN} / \mathrm{CVH}$

108. Condensation

$\mathrm{CVH} / \mathrm{RN}$

$\mathrm{CVH} / \mathrm{RN}$

106. Pressure Suppression System

77. High-Pressure Melt. Ejection

$\mathrm{RN} / \mathrm{CVH}$

82. Fragmentation

FDI

83. Oxidation

FDI

84. Fission Product Release

FDI

FDI

78. Ex-Vessel Hydrodynamics

89. Mixing and Transport of Noncondensable Gases

92. Heat Transfer to Containment and Structures

133. Heat-Transfer Mode from Debris/Melt

136. Convection

137. Radiation

$\mathrm{CVH} / \mathrm{FL}$

$\mathrm{CVH} / \mathrm{FL}$

$\mathrm{CVH} / \mathrm{HS}$

CORCON

CORCON

138. Direct Contact

CORCON

CORCON

134. Direct Energy Transfer (from CCI Gases)

135. Heat Transfer from Gas to Structures

93. Stratification

CORCON

$\mathrm{HS} / \mathrm{CVH}$

94. Natural Circulation and Mixing

$\mathrm{CVH}$

$\mathrm{CVH}$

90. Engineered Safety Features

ESF

95. Suppression Pool

$\mathrm{CVH} / \mathrm{F}$ 
96. Containment Sprays

91. Containment Failure Modes

97. Local Leak Failure Mode

98. Massive Rupture Failure Mode

79. Combustion Processes

85. Ignition

86. Propagation

80. Delayed Release of Fission Products

87. Revaporization After Vessel Breach

88. Release from Water Pools

81. Core-Concrete Interactions

109. Chemical Reactions

110. Debris Spreading

116. Flooded CAV

117. Dry CAV

111. Heat Transfer to Concrete

118. Lateral Heat Transfer

119. Downward Heat Transfer

112. Eutectics, Melting, and Freezing $b$

113. Upward Heat Transfer

120. Radiation/Convection

121. Heat Transfer to Water

130. Heat-Transfer Modec

131. Crust Formation and Stability

132. Bubble Agitation and Entrainment Effects

114. Concrete Response

122. Degassing

123. Ablation

CORCON

115. Fission Product Release

124. Release Mechanisms

125. Transport and Deposition

126. Containment

128. Natural Removal (Condensation/Evap.)

129. Spray Removal

127. Pressure Suppression System
CVH/FL/ESF

$\mathrm{CVH} / \mathrm{FL} / \mathrm{HS} / \mathrm{CF} / \mathrm{FL}$

$\mathrm{CF} / \mathrm{TF}$

$\mathrm{CF} / \mathrm{TF}$

BUR

BUR

BUR

$\mathrm{RN} / \mathrm{CVH} / \mathrm{FL} / \mathrm{HS}$

RN/CVH/FL/HS

$\mathrm{RN} / \mathrm{CVH} / \mathrm{FL}$

CAV

CORCON/VANESA

CAV

CORCON

CORCON

CORCON

CAV/MP

CORCON

CORCON

$\mathrm{CVH}$

$\mathrm{CVH}$

CORCON

CORCON

CORCON

CORCON

RN

RN

RN

$\mathrm{RN} / \mathrm{CVH}$

$\mathrm{CVH} / \mathrm{RN}$

$\mathrm{RN} / \mathrm{CVH}$

a Other phenomena include reflood hydrodynamics and heat transfer at every interval including ex-vessel.

b Other phenomena include dryout and remelting.

c Other phenomena include debris bed dryout and remelting. 


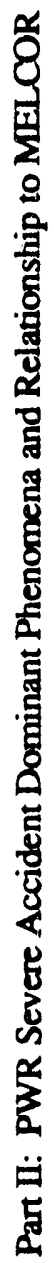

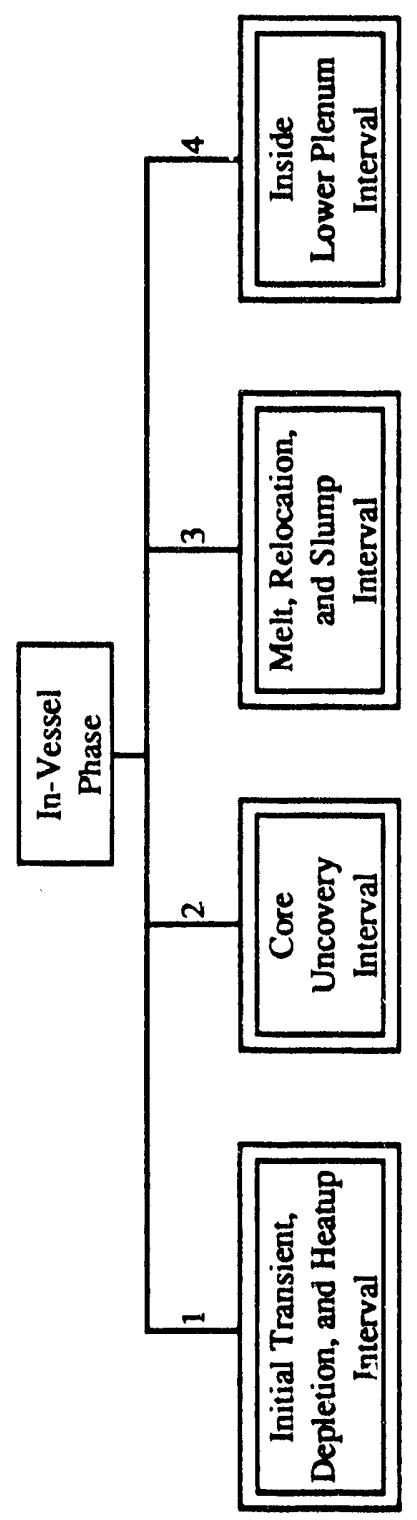




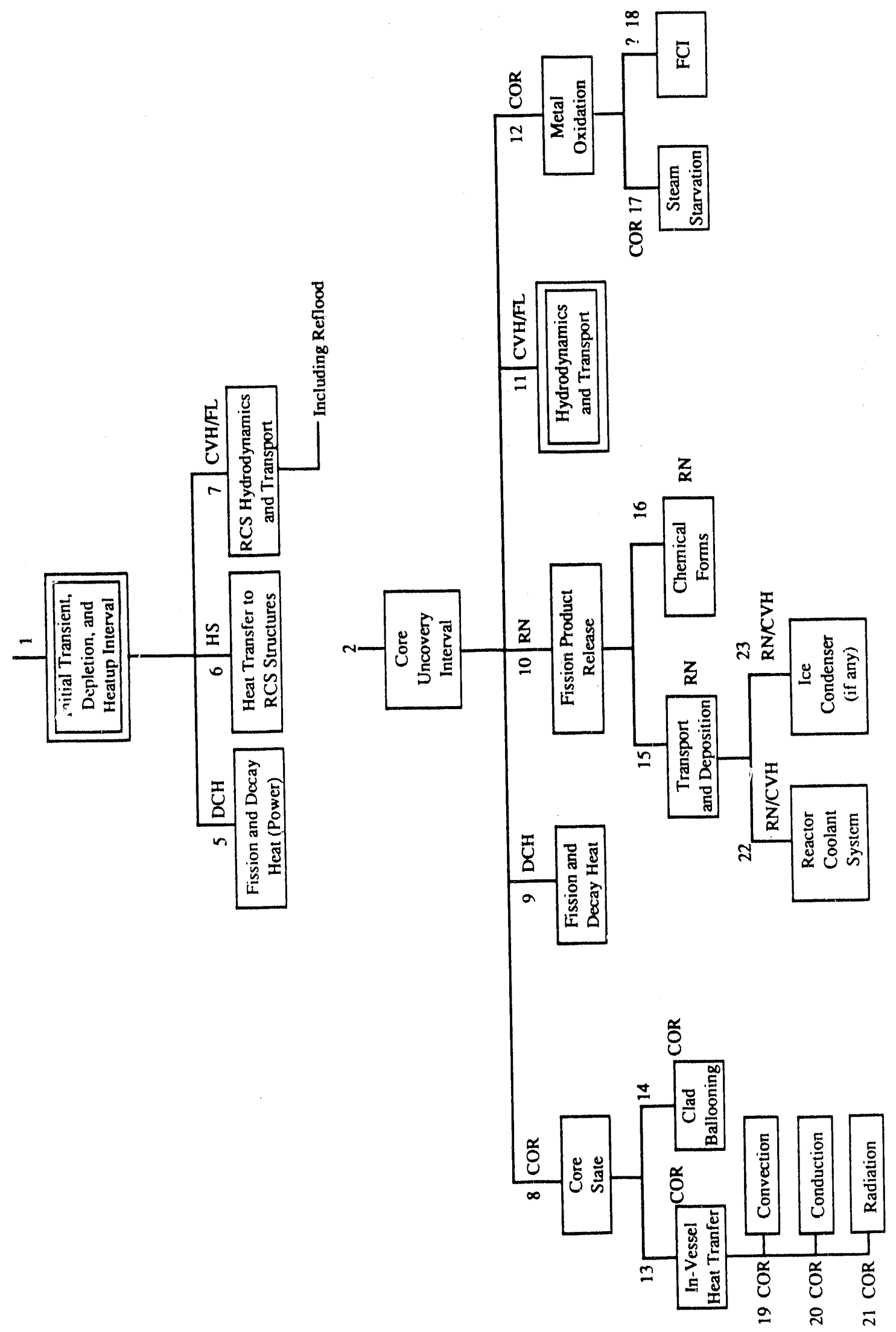

C-19 


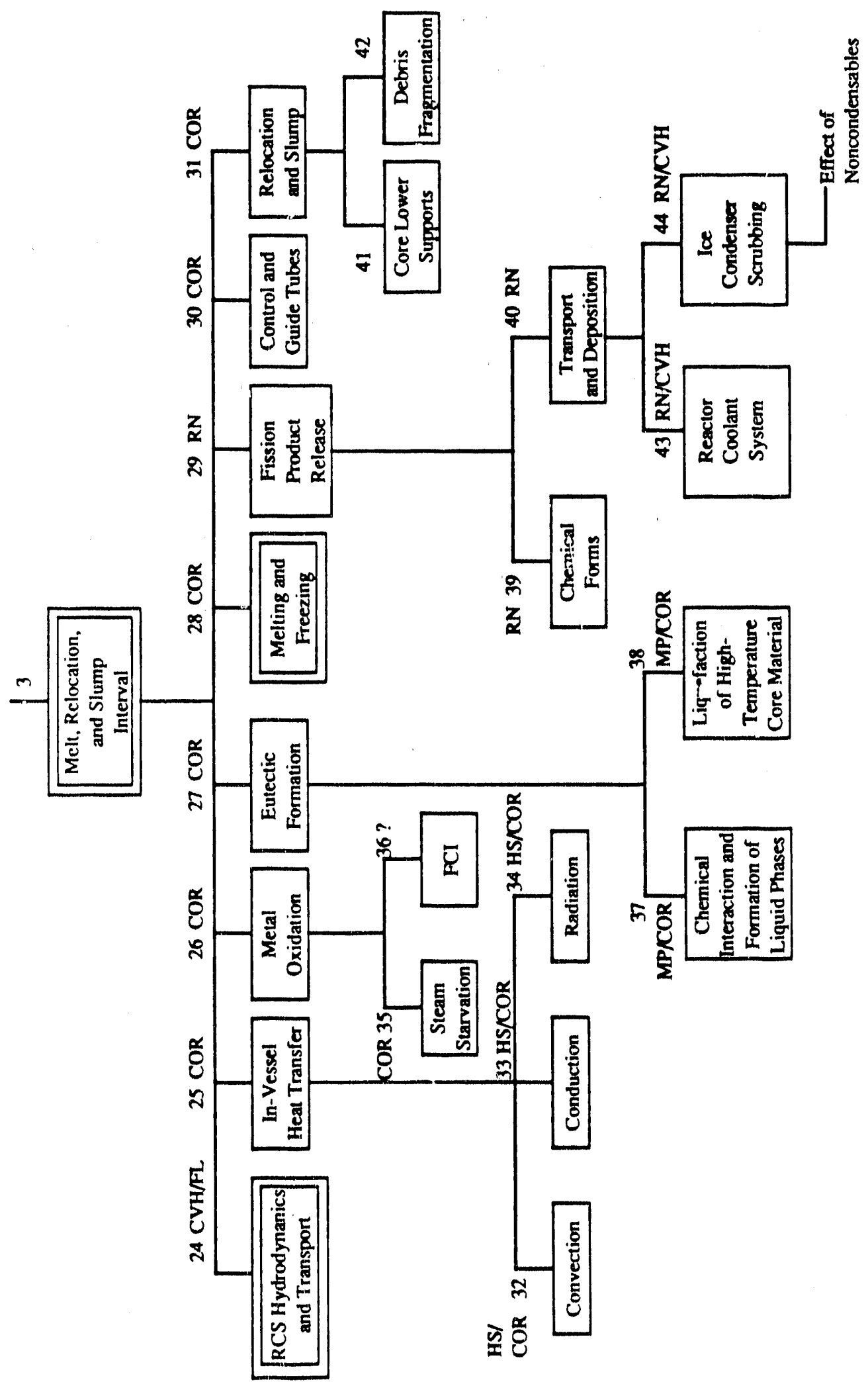

C- 20 


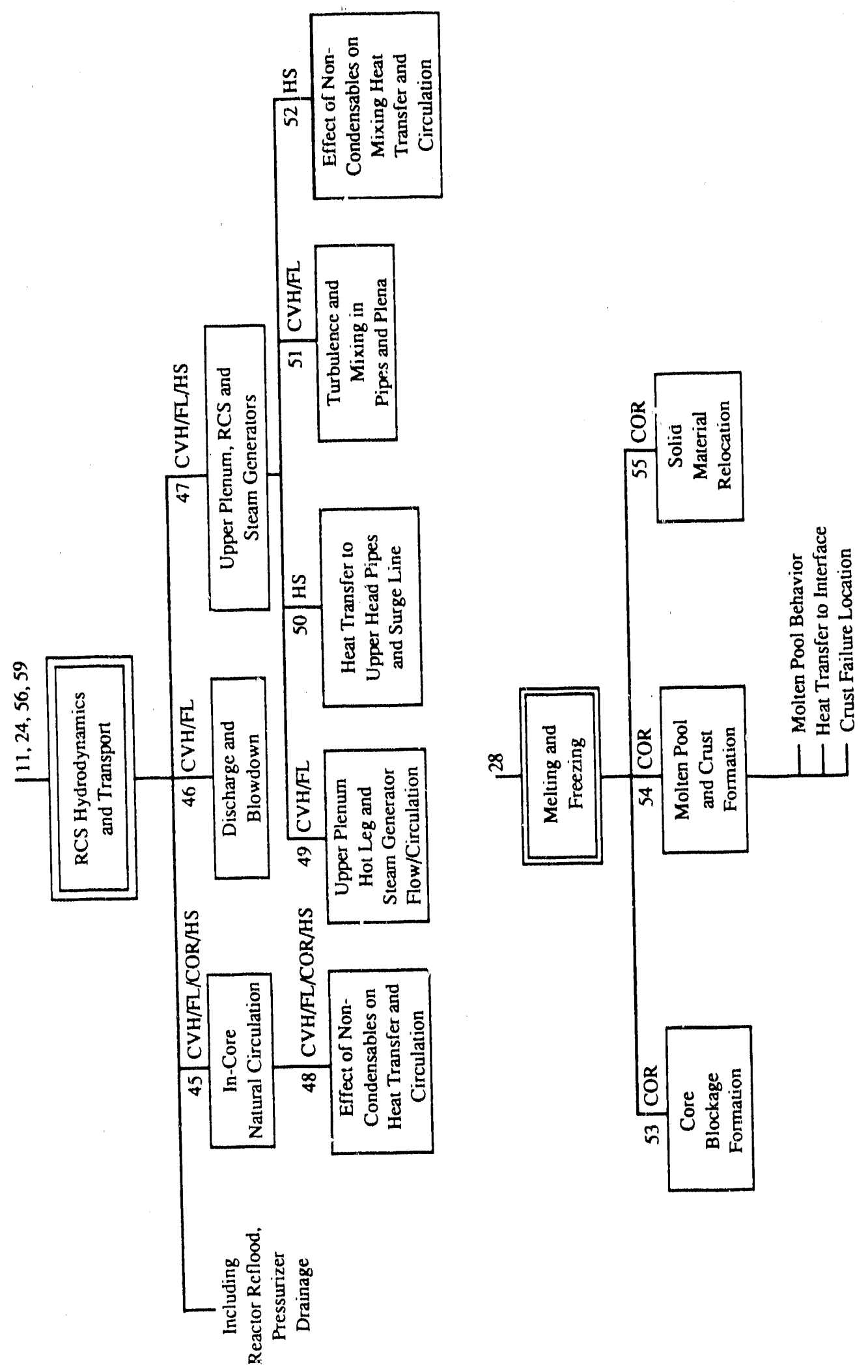




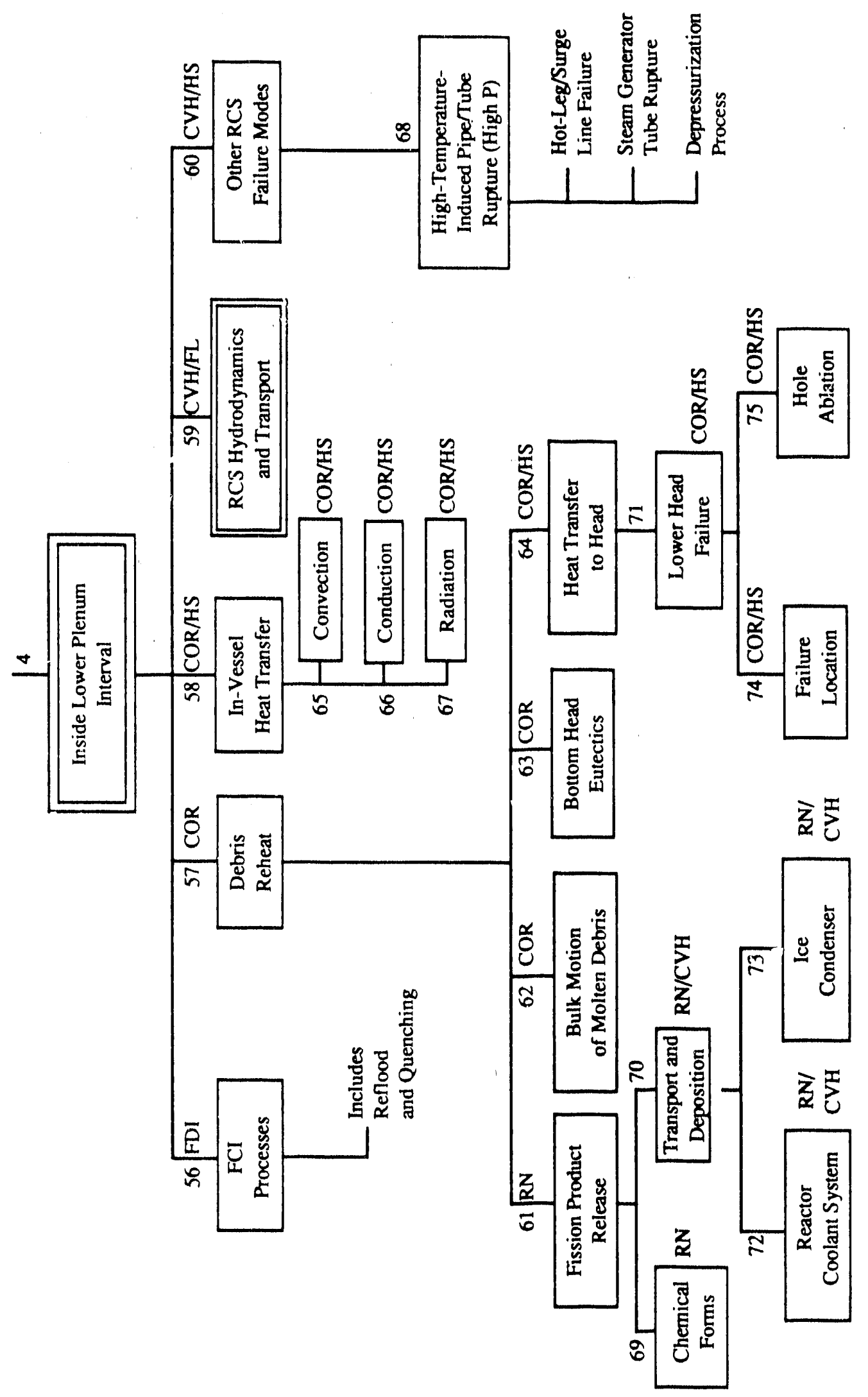




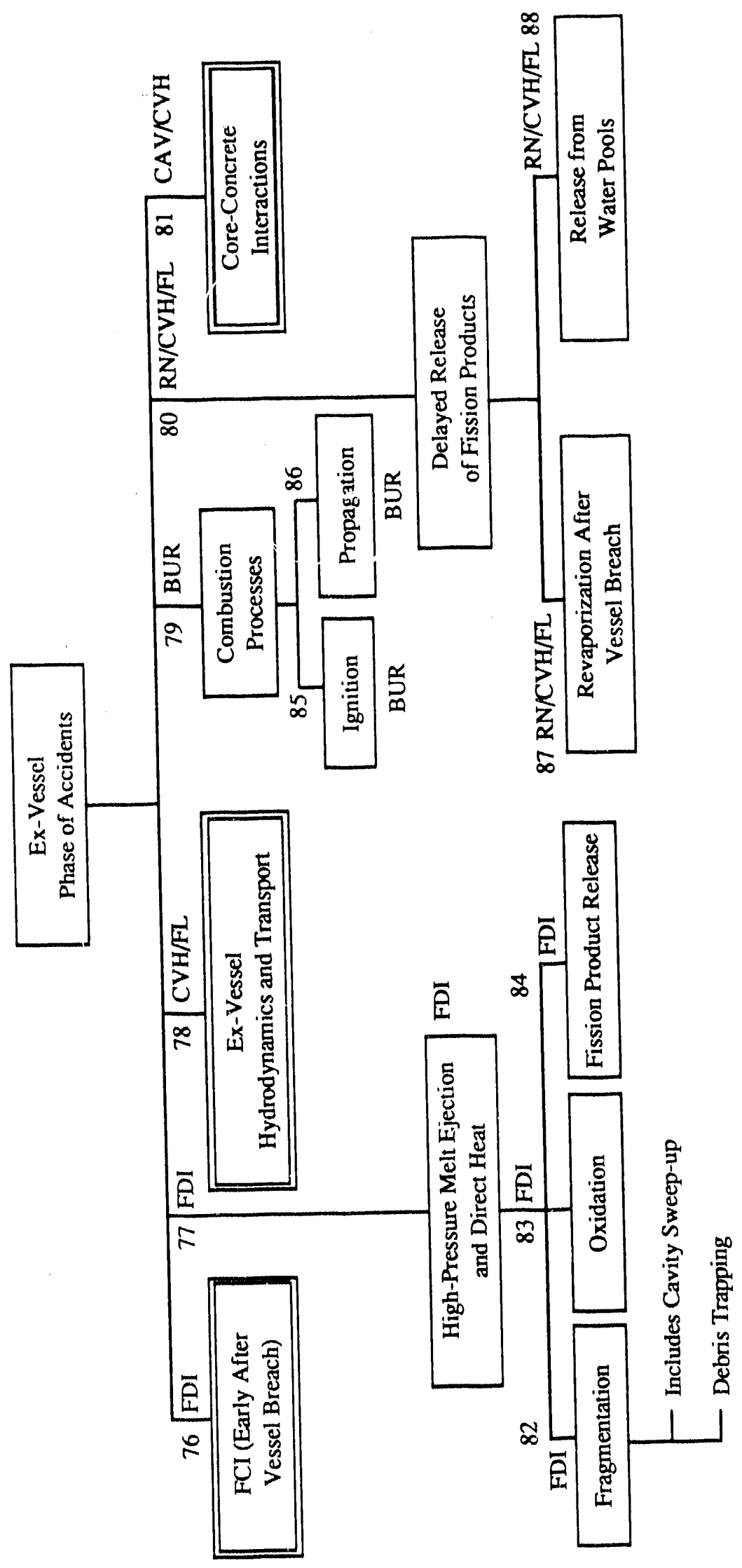

C-23 


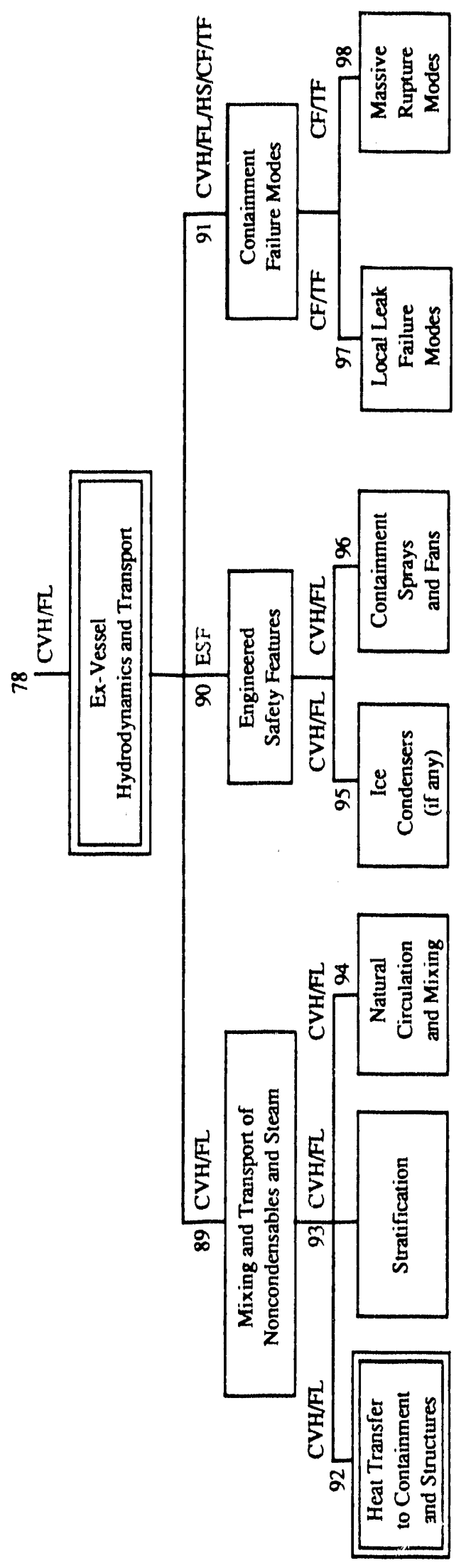

C- 24 


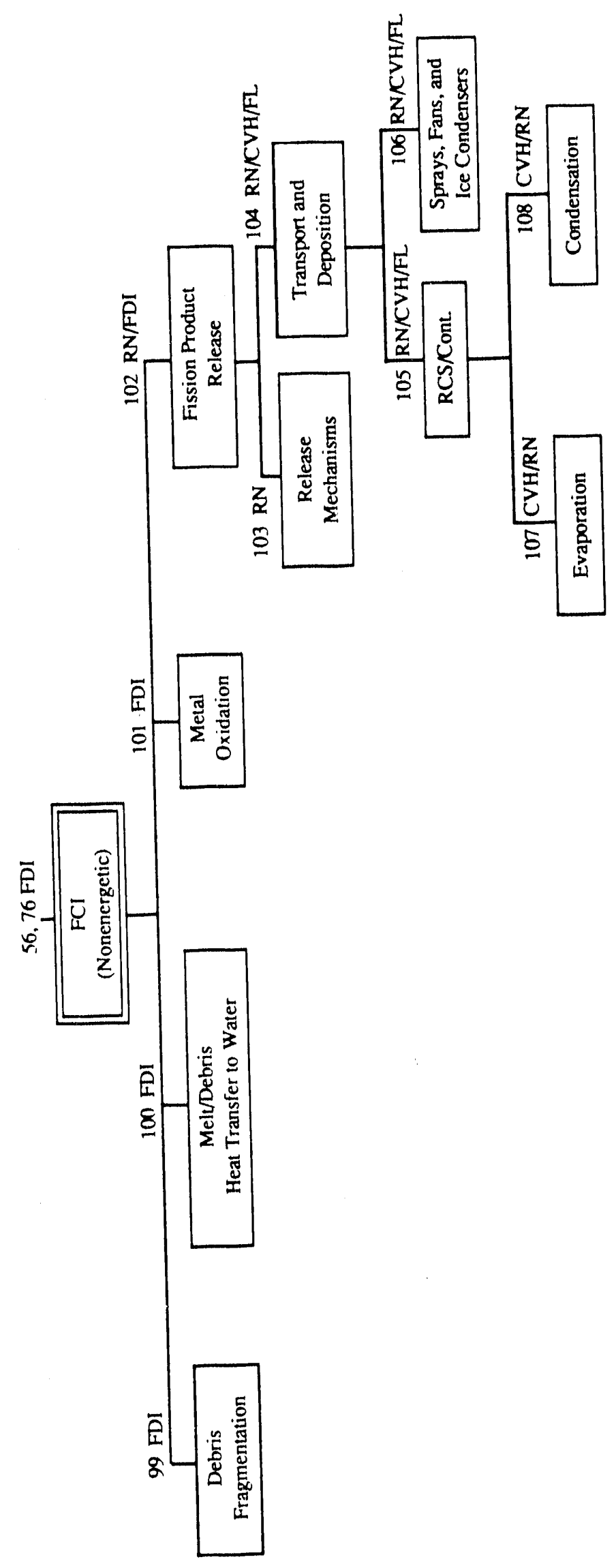




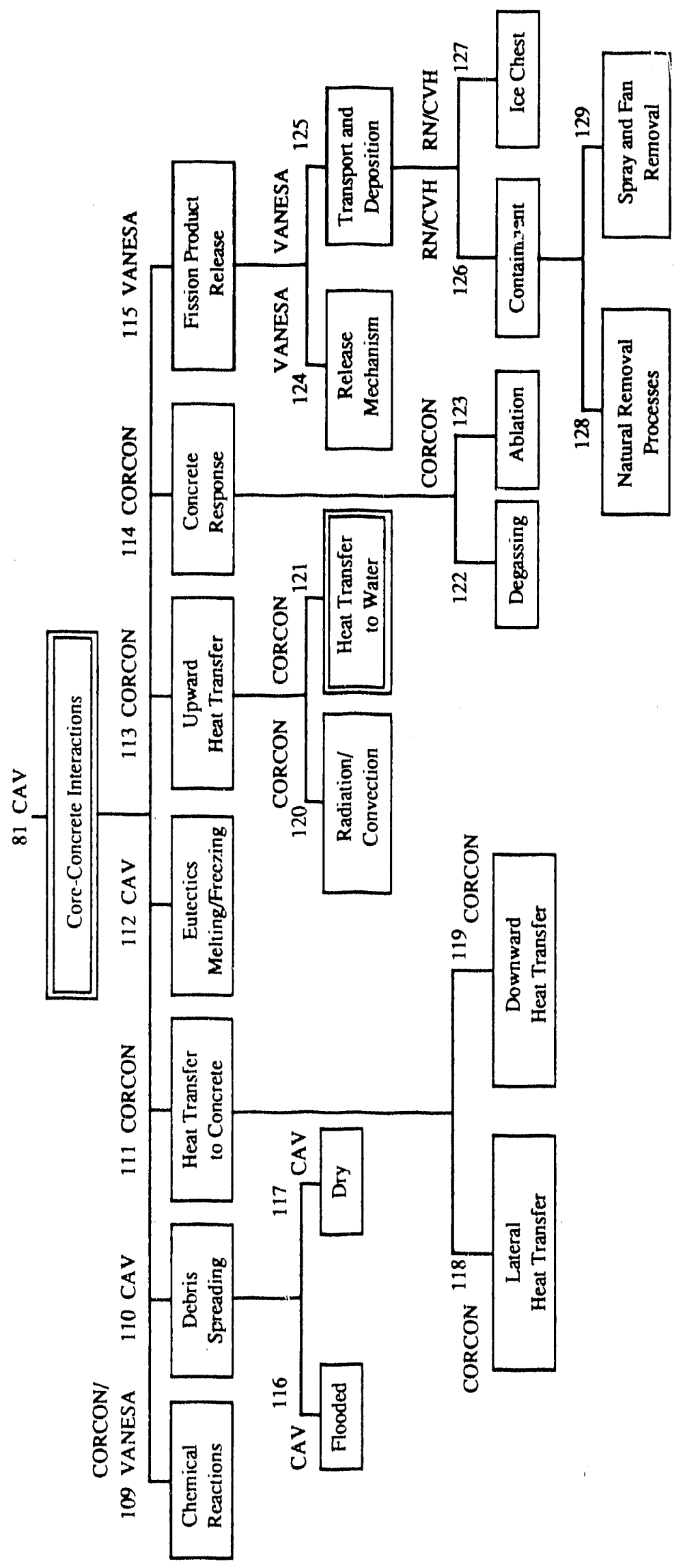



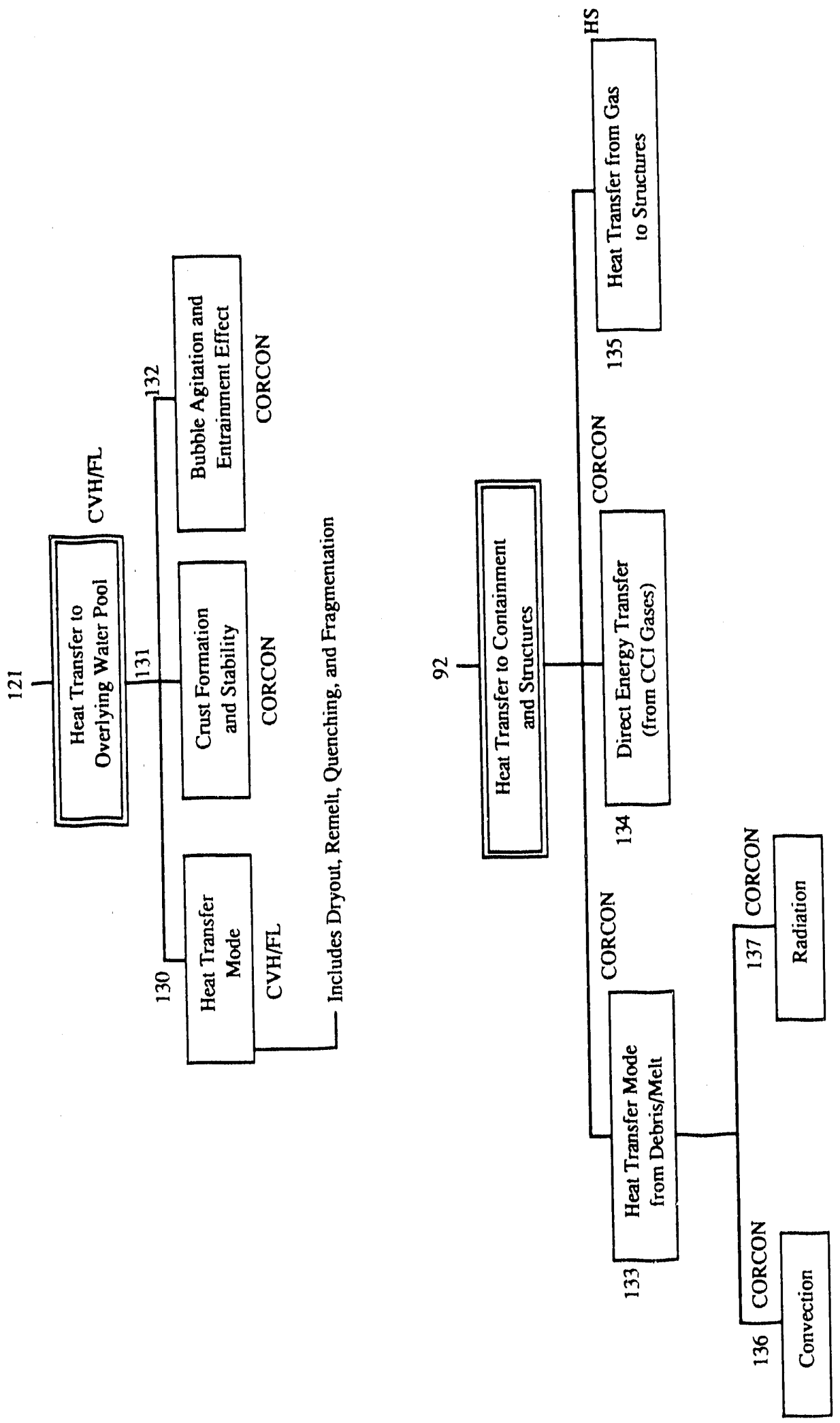


\title{
APPENDIX D
}

\author{
Hydrodynamics Behavior (CVH, FL) Packages
}

by

Mohsen Khatib-Rahbar, Dennis Liles, and Vijay K. Dhir

\section{D.I. MODEL DESCRIPTION AND PEDIGREE}

\section{D.1.1. Overview}

In MELCOR, the thermal-hydraulic processes are modeled by the Control Volume Hydrodynamics (CVH) and Flow Path (FL) packages (Refs. D-1 and D-2), while the thermodynamic calculations are performed within the Control Volume Thermodynamics (CVT) package. The $\mathrm{CVH} / \mathrm{FL}$ packages are based on a highly versatile architecture and a general control volume hydrodynamic network concept that provide thermal-hydraulic boundary conditions to other MELCOR phenomenological modules.

A general "volume/altitude" and "virtual volume" approach is employed to define, through user input, the control volume geometry. Hence, component and subsystem models must be built in through user input (specific component models do not exist within the code structure).

Control volumes are interconnected via "flow paths" inrough which hydrodynamic material may pass without any residence time (assumption of negligible volume). Flow path area can be modified by input to model valves, obstructions, etc. The material and energy contents of both coolant and noncondensable gases are assumed to reside within control volumes.

The FL connections to CVH are referred to as junctions, which are characterized by an elevation and a junction opening height. The void fraction is determined by flow direction and relative position of junction opening and pool surface (fraction of opening height occupied by atmosphere). This can create difficulties for vertically stacked control volumes.

Mass and energy sources and sinks are treated as boundary conditions to CVH/FL (interaction models are not present within the $\mathrm{CVH}$ package). This includes decay heat, heat from structures, water from condensation and evaporation on structures, and nonconderisable gas sources from core-concrete interactions (CAV), oxidation, and other sources.

In $\mathrm{CVH} / \mathrm{FL}$, hydrodynamic materials are assumed to separate by gravity into a lower pool region (which may contain stearm bubbles but not noncondensables), and an overlying atmosphere (which may contain liquid droplets, gases, and vapor). The pool and atmosphere velocities and directions may be different.

The mass exchange models include options for (1) a thermal and mechanical equilibrium model that assumes the same pressure and temperature for both pool and atmosphere, and (2) a thermal nonequilibrium model that assumes the same pressure but different temperatures for pool and atmosphere (vapor superheat and liquid subcooling).

\section{D.1.2. Conservation Equations}

The equations solved by CVH are the conservation of mass and energy within each control volume, and neglect the kinetic energy term of the energy balance equation.

The conservation of momentum for pool and atmosphere, separately, is solved for each flow path (FL package). The momentum balance equations include inertial, gravitational, frictional, and other head losses, while the spatial acceleration losses have been ignored. It is important to note that, in $\mathrm{CVH} / \mathrm{FL}$, the old time velocity in the backward Euler time differencing is not the velocity from the previous time step, but an ad hoc relationship involving the old time 
velocities and void fractions that attempts to preserve the volume flux of each component and the relative velocity. This further (plus the absence of spatial derivative flux term) relaxes the idea of momentum conservation. In addition, this type of averaging can introduce additional time-step sensitivities.

The basic hydrodynamics methods embodied within $\mathrm{CVH} / \mathrm{FL}$ are closer in principle to those of RELAP4 than the TRAC or RELAP5 codes. The CVH/FL models are an adaptation of the approach employed by HECTR (Ref. D-3) and CONTAIN (Ref, D-4) containment analysis cocies, also developed at SNL.

The current model does not include provisions for simulation of countercurrent (all liquid or all vapor) single-phase flow fields typical of those expected during low flow natural convection conditions within the hot-leg region of PWRs for high-pressure accident sequences (e.g., station blackout). Furthermore, the spatial acceleration terms are also important for prediction of twophase natural convection flow behavior.

\section{D.1.3. Condensation and Evaporation}

In the CVT package, thermal and mechanical equilibrium (equilibrium thermodynamics option) is maintained by instantaneously transferring mass and energy between pool and atmosphere. If the thermal nonequilibrium option for a volume containing both pool and atmosphere is selected, CVT will transfer mass and energy equivalent to the pressure times change of volume (PdV) work done by one region (pool/atmosphere) on the other (Refs, D-1 and D-2). The mass and energy transfer rate is calculated based on a heat-transfer/mass diffusion theory model.

The rate of heat and mass transfer at the pool surface is calculated by simultaneous solution of heat- and mass-transfer equations, assuming saturation at the pool/atmosphere interface. Certain types of phenomena such as phase change with superheated liquid cannot be modeled directly. This is probably not a major limitation over long time intervals for the type of usage appropriate for MELCOR.

The energy transfer rates consider thermal radiation and convection processes leading to evaporation or condensation at the surface. Empirical heat-transfer correlations for forced and natural convection are used. For condensation into a quiescent pool of subcooled liquid (e.g., in ice-condenser containments, where a steam atmosphere can exist over a large pool of subcooled liquid), the present model calculates energy, ansfer by natural convection from a stably stratified warm saturated liquid layer (at the interphase) to a large pool of subcooled liquid, which is physically incorrect and can lead to substantial overpredictions in steam condensation rates. Under conditions of vigorous mixing, forced convection effects dominate and this modeling error is less significant.

The forced convection correlation (for horizontal stratified flows) is taken from the TRAC code, whereas the free convection correlations are based on laminar and turbulent correlations for horizontal surfaces. Justifications are not provided for the appropriateness of these correlations to conditions foreseen during severe accidents, including experimental bases, range of applicability, etc. These correlations were mostly developed for geometries and conditions less complicated than those encountered in reactors and during severe accident conditions. For example, Eqs. (5.1.21) through (5.1.23) of Ref. D-1 are more directly applicable to volumes with sinall (L/D) aspect ratios (e.g., reactor vessel, pressurizer, suppression pool, etc.) than to volumes with large aspect ratios (e.g., steam generator tubes, etc.). Furthermore, the following CVH definition of the characteristic dimension (Ref. D-1),

$$
X=\min \left(D_{S}, L\right)
$$

can produce inconsistencieg with the characteristic dimensions of the listed correlations. However, calculated heat-transfer coefficients will not be notably altered, because of their weak dependence on the characteristic length. This is not expected to be of major significance for simulation of 
severe renctor acoldents.

\section{D.1.4. Bubble Rise and Phase Separation}

In a saturated pool, bubble rise is treated using a very simple model, where the void fraction is assumed to vary linearly from zero at the bottom to twice its volume average value at the surface. This profile is assumed to remain time invariant. Furthermore, bubbles are assumed to rise at a constant velocity of $(0,30 \mathrm{~m} / \mathrm{s}$. In addition, an upper limit of $40 \%$ is also imposed on the pool void fraction, beyond which additional vapor is added to the atmosphere. Vapor bubbling up through a pool from a volume below is not accounted for. Noncondensable gas is also not accounted for in the bubble model.

Precipitation of water droplets suspended in the atmosphere (fog) is treated as part of the aerosol module (RN package) using the MAEROS model. Since the CVH package does not include any mechanisms for fog removal, an upper limit of $\left(0.10 \mathrm{~kg} / \mathrm{m}^{3}\right.$ has been imposed on fog density, beyond which excess water droplets are transferred to the pool. The impact of fog precipitation modeling assumptions on CVH-calculated results has not been demonstrated.

\section{D.1.5. SPARC Bubble Physics}

An optional bubble (water vapor, noncondensable gases, and fog droplets) pool interaction model, based on the physics of the SPARC (Ref, D-5) code is also included in the CVH package. In this model, gas streams entering a pool of water are assumed to break up into a swarm of bubbles, thermally equilibrating with the pool, and salturating with water vapor at local conditions. This model is implemented into the CVH package through modifications in mass and energy terms of the control volume equations. The impact and applicability range of this model under various geometric and flow regime conditions has not been demonstrated. This model in conjunction with the $R N$ package is used to predict retention of fission product in water pools. Calculations performed recently with MELCOR (Ref. D-10) have shown substantial underpredictions in decontamination fuctors for BWR suppression pools.

\section{D.1.6. Pool/Atmosphere Force}

The momentum transfer between pool and atmosphere phases vithin a flow path is important both in entrainment (cocurrent flows) and in flooding or countercurrent flow limitations (CCFL),

The present MELCOR model is based on an approximation to a Wallis flooding correlation, and thus avoids inclusion of detailed flow regime maps. 'The same simple approach is also used to model flooding controlled pressurizer drainage for PWR accidents.

Universal application of this model to all geometries and flow regime conditions is not justifiable, becaluse it cannot differentiate between different types of flow paths. Furthermore, the impact of the present simplified approach on MELCOR predictions under various flow regimes and geometrical conditions has not been fully assessed.

\section{D.1.7. Pumps and Fans}

The two available pump/fan modeling options include (1) a simple parabolic head flow relationship for constant speed pumps/fans, and (2) user supplied inputs of homologous characteristics through Control Functions (CFs). In either case, the supplied head is provided as an explicit boundary condition to the FL package momentum equation. 
The code does not model the angular momentum equation for the impeller dynamics, and as such, it cannot correctly simulate the pump/fan dynamic behavior. Instead, it relies on the user-. supplied behavior of pumps/fans as a function of time.

Pump behavior is generally important during the very early stages of accidents or for accidents involving pump restart. For these cases, other available codes such as RELAP5 and TRAC can be used to assess the pump behavior. Therefore, iack of detailed pump/fan models is not believed to be a major deficiency for MEL_COR simulation of severe accidents.

\section{D.1.8. Critical Flow}

The critical flow in each flow path is always calculated and compared with the solution of the flow path momentum equation. When the $\mathrm{CVH} / \mathrm{FL}$-calculated flow rate is higher than the critical value, it is set equal to the critical flow. This "test is bypassed if neither the pool velocity

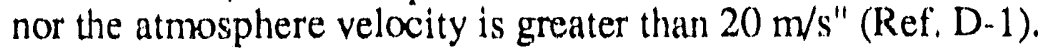

The CVH/FL critical flow models include

$$
\text { Pool Flow Only: } \quad \text { Extended Henry-Fauske (Subcooled) }
$$
Moody (Saturated)

Atmosphere Flow Only: Ideal Gas Model

Pool and Atmosphere Flow: Weighted Average of Above (with weighing designed to approximate Moody's model)

The $\mathrm{CVH} / \mathrm{FL}$ critical flow approach appears reasunable for application to severe accident analysis.

\section{D.1.9. Other Models}

\section{D.I.9.1. Valves}

Valves may be included in any flow path to force adjustments in the fractional opening of the flow path areas, using the Tabular Function (TF) and/or the Control Function (CF) options. This provides a highly general and versatile approach for inclusion of any type of valve based on plant-specific design and operating data. The TF and/or CF supplied area is treated as an explicit input to the flow path momentum equations. This can potentially lead to inaccuracies and time-step dependencies in the $\mathrm{CVH} / \mathrm{FL}$-calculated results.

\section{D.1.9.2. Wail Friction}

The wall friction is calculated based on laminar and turbulent relationships for circular pipes, with interpolation in the critical transition flow regime $(2000 \leq \operatorname{Re} \leq 5000)$.

The single-phase and mixture Reynolds numbers use the local segment velocities obtained on the basis of continuity equation for steady, incompressible flow at each time step.

The mixture (two-phase) friction factor is assumed to have the same Reynolds number dependence as single-phase flow, using the two-phase mixture Reynolds number and viscosity as defined by Beattie and Whalley [Eqs. (5.4.6) and (5.4.7) of Ref. D-1]. Justifications for selection of this approach over traditional methods have not been provided.

The impact of blockage formation within flow paths (i.c., core flow channels) resulting from degradation of fuel elements, on the flow path resistance is ignored; nevertheless, channel flow area can be changed via CFs. Increased flow resistance as a result of debris/melt blockage following core degradation leads to flow diversion to unblocked regions (PWRs and BWRs after water seal clearance) with potentially significant impact on hydrogen generation, melt progression, and in-vessel natural circulation. 


\section{D.2. IMPLEMENTATION WITHIN MELCOR}

The CVH/FL conservation equations are changed to linearized semi-implicit finite difference equations, and solved together (but not simultaneously). The interface boundar conditions of $\mathrm{CVH} / \mathrm{FL}$ modules and the other MELCOR phenomenological modules are treated explicitly. These include external sources, heat fluxes from Heat Structures (HS module) and Core (COR module), fog precipitation ( $R N$ module), etc.

The CVH/FL time step is increased within the CVH/FL time-stepping scheme, as well as within the overall MELCOR time-stepping framework. The numerical integration techniques currently implemented within the $\mathrm{CVH} / \mathrm{FL}$ packages appear reasonable. However, the explicit coupling between CVH, COR, RN, and other MELCOR packages could enhance the potential for numerical instabilities reported by several users (Refs. D-6 and D-7) and discussed in the next section.

Occurrence of "sudden events" resulting from "logical" arguments and other physical phenomena (i.e., failure of an oxide shell in the COR package, depressurization, pressuization, valve action, etc.) can exacerbate the problem of numerical instabilities. Explicit coupling between $\mathrm{CVH} / \mathrm{FL}$ and valves as well as $\mathrm{CVH} / \mathrm{FL}$ and control volume leakage may also produce numerical instability problems for CVH/FL.

\section{D.3. RESULTS OF PARAMETER AND SENSITIVITY STUDIES}

Parameter and sensitivity studies specifically aimed at assessing the CVH/FL packages are very limited. Most of the sensitivity studies are performed, either as part of the experimental benchmarking activities (discussed in next section), or full-plant thermal-hydraulics and source term simulations.

A number of sensitivity calculations have been performed by Martinez (Ref. D-6) as part of the MELCOR posttest calculation of the HDR experiments. These include sensitivities to convective velocities, flow loss coefficients, and computer type. Time step, control volume nodalization, and other sensitivities have also been performed by Robertson (Ref. D-7) as part of the validation exercise for the BMC-F2 experiment.

Both of these studies have attempted to simulate the transport of gases in a large-scale multicompartment containment, under conditions prototypic of severe reactor accidents. Table D-I summarizes the results of these parameter and sensitivity studies.

It is seen that the CVH/FL packages are also susceptible to the same type of problems that are inherent in most computer codes with a similar control volume approach, namely,

- difficulties in defining control volume velocities for use in closure relations,

- difficulties in defining characteristic lengths, flow paths, and loss coefficients in large compartments that are not well represented by "tank-pipe" configurations,

- difficulties with the so-called "numerical diffusion" associated with the well-mixed assumption of the control volume approach, and

- difficulties in predicting temperature stratification (with and without noncondensable gases). 
Table D-I

Summary of CVH/FL Parameter and Sensitivity Studies

\begin{tabular}{|l|l|}
\hline Parameter/sensitivity & Impact on Calculated Results/Conclusions \\
\hline $\begin{array}{l}\text { Definition of convective velocity } \\
\text { (Ref. D-6) }\end{array}$ & $\begin{array}{l}\text { Significant impact in the blowdown (transient)phase } \\
\text { - Insignificant impact in cooldown/quasisteady phase } \\
\text { Intra-compartment recirculation effects important }\end{array}$ \\
\hline Loss coefficients (Ref. D-6) & $\begin{array}{l}\text { Some impact, especially in the transient phase } \\
\text { - Insignificant in terms of inter-compartment mixing }\end{array}$ \\
\hline Computer type (Ref. D-6) & $\begin{array}{l}\text { VAX-8700 and CRAY X-MP/416 results were } \\
\text { identical }\end{array}$ \\
\hline & $\begin{array}{l}\text { Major impact on prediction of stratification } \\
\text { Major impact on condensation }\end{array}$ \\
\hline Time-step size (Ref. D-7) & $\begin{array}{l}\text { Numerical instabilities at large maximum time steps, } \\
\text { resulting from explicit coupling of condensation } \\
\text { heat and mass transfer to the CVH/FL package }\end{array}$ \\
\hline & $\begin{array}{l}\text { Major impact on total mass and energy balance } \\
\text { (total containment pressure) }\end{array}$ \\
\hline Leakage to outside (Ref. D-7) & $\begin{array}{l}\text { More significant for side and dead-end } \\
\text { compartments }\end{array}$ \\
\hline & $\begin{array}{l}\text { Significant impact on prediction of gas transport } \\
\text { Impact more pronounced for dead-end volumes }\end{array}$ \\
\hline & $\begin{array}{l}\text { Well-mixed assumptions of control volume } \\
\text { approach require some intuition on the part of code } \\
\text { users }\end{array}$ \\
\hline
\end{tabular}

More recently, extensive sensitivity studies have also been reported for typical BWR several accident sequences. These plant-specific integral calculations have also shown a very strong dependence on maximum time step, computer type, compiler option, modeling options, and parametric inputs (Refs. D-8 through D-10).

The MELCOR numerical instabilities have shown to produce results which are often difficult to discern from actual physical trends, and they appear to be more pronounced for integral plant calculations (Refs. D-8 through D-10).

\section{D.4. RESULTS OF BENCHMARKING/VALIDATION ACTIVITIES}

A number of separate effects and integral MELCOR calculations have been performed as exemplified by Refs. D-6 through D-9 and D-11 through D-15. These studies have been aimed at testing either the $\mathrm{CVH} / \mathrm{FL}$ package for application to separate-effects experiments (Refs. D-6, D-7, and D-13), or as part of an integral phenomenological assessment and full-plant simulation (Refs. D-8, D-9, D-11, D-12, D-14, and D-16).

Benchmarking studies performed to date have demonstrated that judicious application of the $\mathrm{CVH} / \mathrm{FL}$ packages to steam and noncondensable gas transport problems in large, multicompartmental volumes (Refs. D-6, D-7, and D-13) can reproduce major phenomenological trends of significance during severe reactor accidents. 
Boucheron and Kelly (Refs. D-11 and D-12) have also attempted to simulate the TMI-2 accident using MELCOR. Results of this integral MELCOR calculation showed reasonable agreements with the data for the initial transient heatup and depletion, and the core uncovery intervals (start of core damage). Difficulties in preventing pressurizer drainage were also reported.

In addition, as part of the present review, the $\mathrm{CVH} / \mathrm{FL}$ was used to simulate several simple and well-characterized problems to develop a better appreciation for the $\mathrm{CVH} / \mathrm{FL}$ hydrodynamics models and their numerical implementation into the MELCOR architecture.

\section{D.4.1. Vessel Blowdown}

A series of medium scale blowdown tests were performed in the early 1980s at General Electric (GE) (Ref. D-17). A schematic of the test apparatus is shown in Fig. D-1. The pressure vessel was $1.10 \mathrm{~m}$ in diameter, $4.3 \mathrm{~m}$ long, and contained a volume of $4.5 \mathrm{~m}^{3}$. The outer walls of the vessel were insulated.

Blowdown tests were conducted with the blowdown line connected to either the top or bottom of the vessel. Figure D-I shows the dip tube inserted so that a top-level blowdown is represented. When its inner tube was removed the test was referred to as a bottom blowdown test. Various diameter venturi were inserted to vary the blowdown rate. Initially saturated water at slightly greater than $7.14 \mathrm{MPa}$ (1000 psia) partially filled the vessel. Saturated steam filled the remainder of the tank.

The first test presented is GE Test $5801-13$. The venturi throat was $5.39 \mathrm{~cm}(2-1 / 8 \mathrm{in}$.) in diameter. Saturated liquid at $7.21 \mathrm{MPa}$ (1060 psia) filled the tank to a height of $1.68 \mathrm{~m}(5.5 \mathrm{ft})$ from the bottom. The dip tube was in place so this represented a top blowdown.

The MELCOR input used two vertical volumes to represent the vessel. The code developers recommended a single volume but it was felt that two provided a better a reactor representation and might show any anomalous behavior associated with the interfacial drag. The nonequilibrium thermodynamics option was evoked. Figures D-2 and D-3 represent the pressure history traces for a choking factor of 1.0 and 0.7 respectively; a flow link connected the upper volumes to the venturi opening. One flow link connected the upper and lower vessel volumes (the MELCOR input deck for this problem is listed in Annex D-1 to this appendix).

The pressure results show good agreement with the test data. In the test, frothing occurs but fails to cover the dip tube opening at any point during this transient, so only vapor escapes the vessel. The data included a mixture level; however, the very crude nodalization and the MELCOR assumptions concerning bubble distributions in a pool render these comparisons meaningless. It should be noted that the break was opened in MELCOP. aft, $5 \mathrm{~s}$ of real time rather than at $\mathrm{t}=0.0$. This produces a 5-s plateau in the computed results. A choking coefficient of perhaps 0.75 might provide optional results.

The code computes slowly as Fig. D-4 indicates. On a CRAY Y-MP the CVH/FL packages consume about $30 \mathrm{~s}$ of CPU time for the $48 \mathrm{~s}$ of real time. The total overhead is large suggesting a good bit of time spent in data management and input/output. As a standard of comparison, TRAC could run a TMI small-break blowdown with approximately 100 control volumes approximately 10 times faster than real time (i.e., real time/CPU time $=10$ ). It could be anticipated that a significant fraction of $\mathrm{CVH}$ computer time is spent evaluating the equation of state.

Figure D-5 shows a calculation with two full height flow links between the vessel volumes. Because MELCOR allows multiple flow paths between mesh cells it was deemed desirable to see what if any effects might occur for this problem. The results for a choking coefficient of 1.0 overlay the one flow link results (Fig. D-2). 


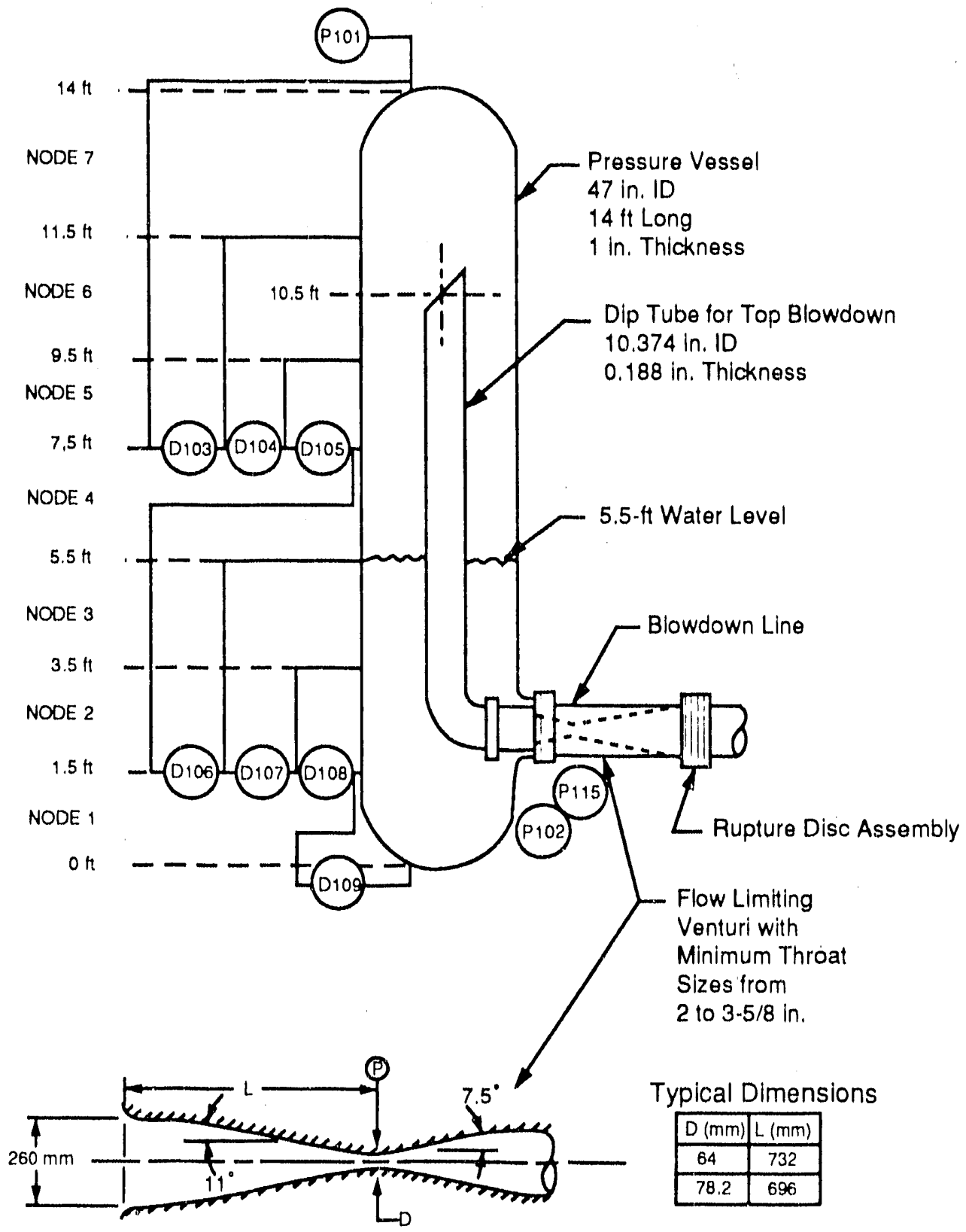

Fig. D-1.

Schematic of GE large blowdown vessel. 


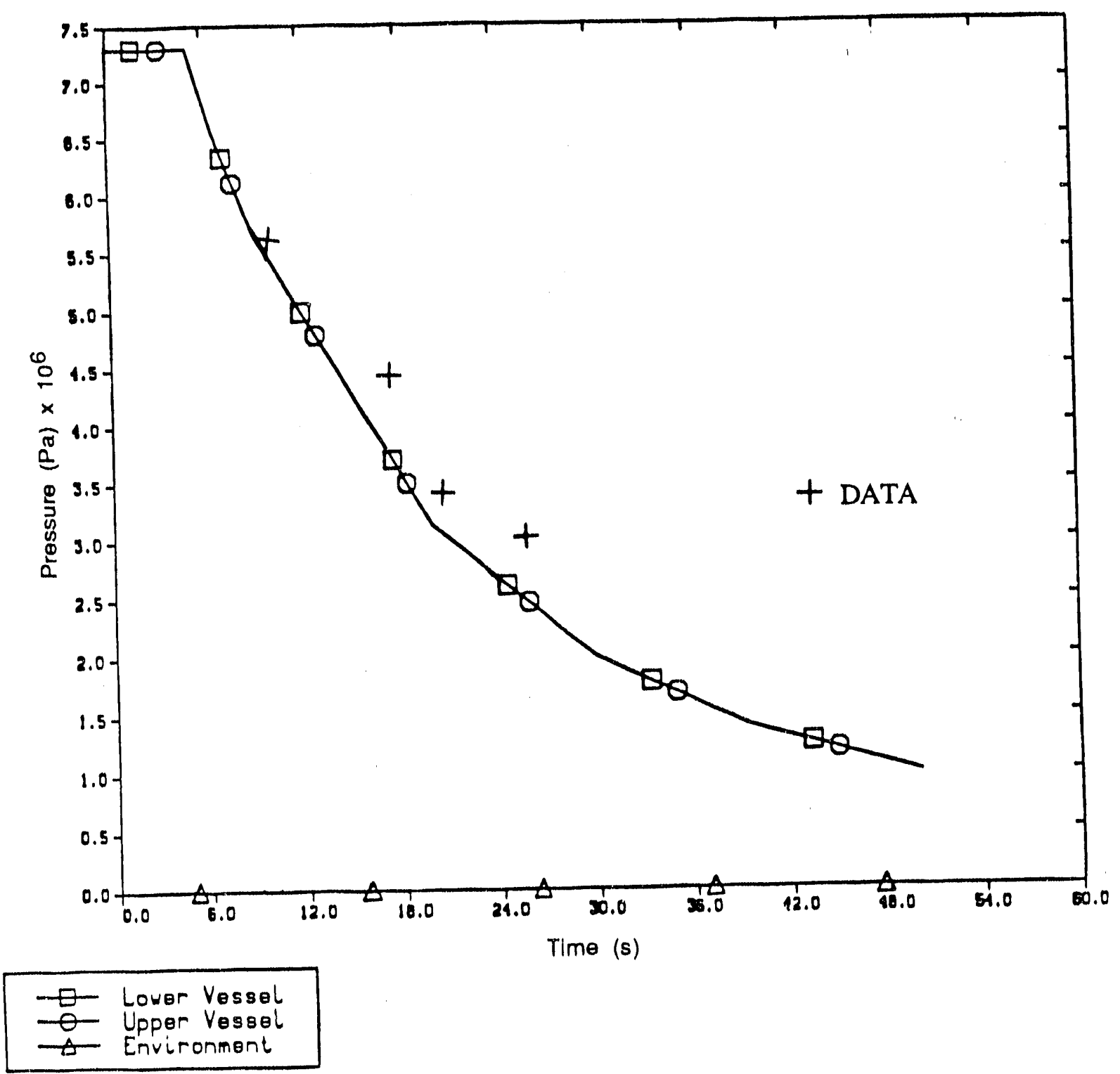

Fig. D-2.

Comparison of MELCOR pressure predictions and GE Test 5801-13 experimental data (choking factor of 1.0 ; maximum time step of $2.0 \mathrm{~s}$ ). 


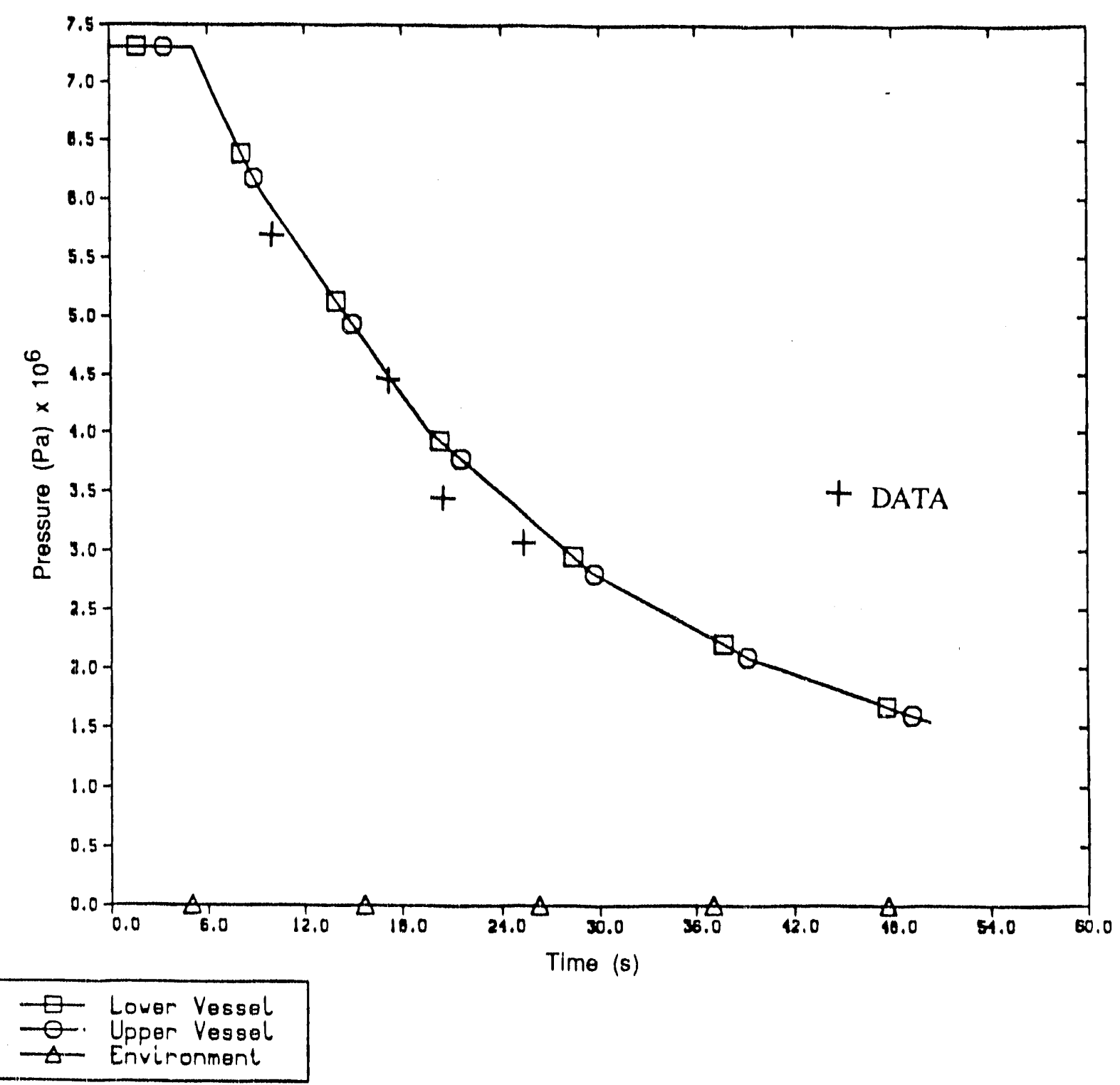

Fig. D-3.

Comparison of MELCOR pressure predictions and GE Test 5801-13 (choking factor of 0.70; maximum time step of $2.0 \mathrm{~s}$ ). 


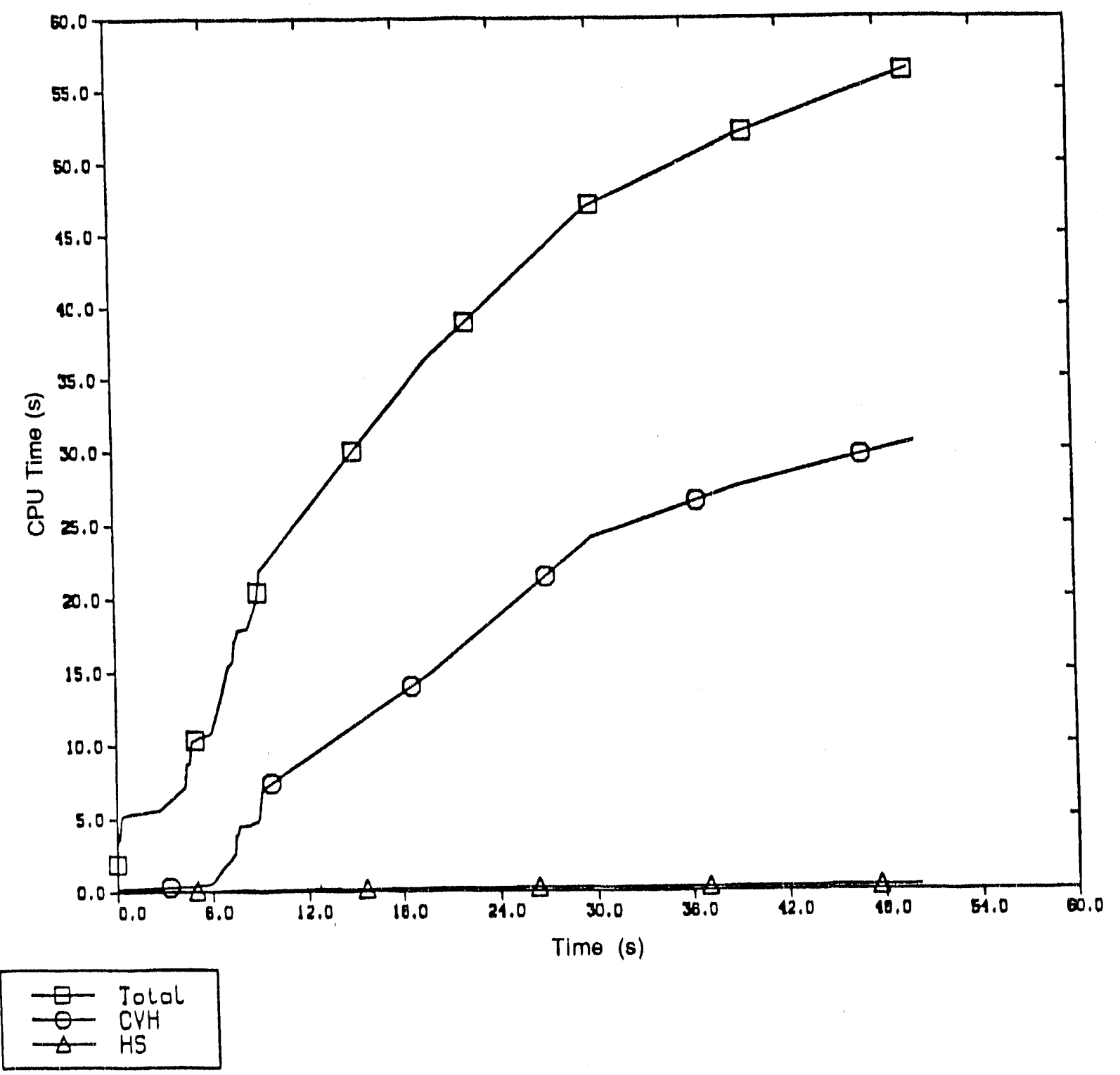

Fig. D-4

CPU time vs real time (choking factor of 1.0; maximum time step of $2.0 \mathrm{~s}$ ). 


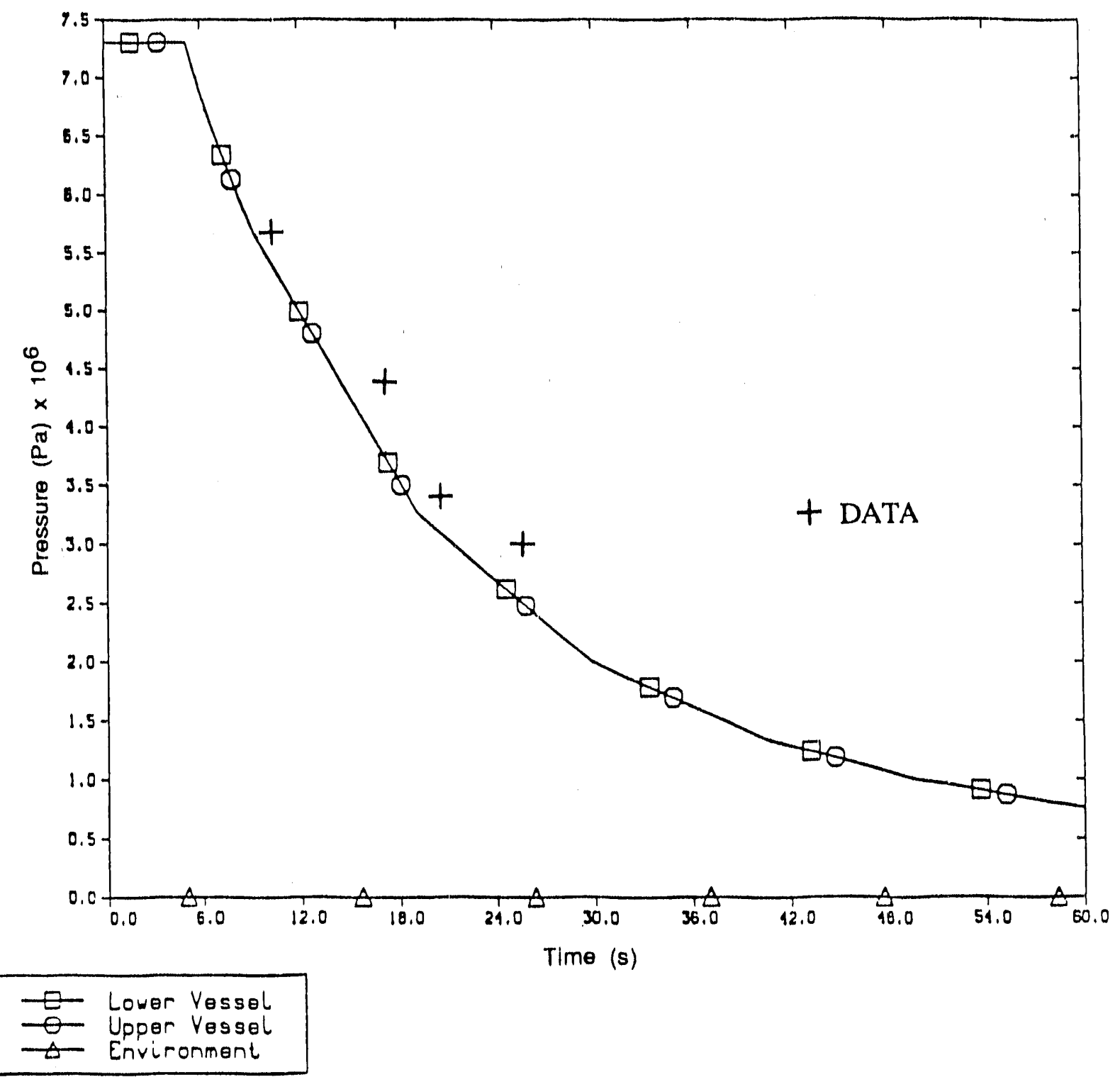

Fig. D-5.

Comparison of MELCOR pressure predictions for (a) one-flow path, and (b) two-flow path link (GE Test 5801-13; choking factor of 1.0; maximum time step of $2.0 \mathrm{~s}$ ). 
A bottom blowdown (GE Test 5803-1) was also simulated with MELCOR. The flow link to the venturi was shifted to the $0.61-\mathrm{m}$ location of the center of the nozzle. The venturi diameter was the same as Test 5801-13; however, the initial water level was $2.29 \mathrm{~m}$ (7.5 ft) high at pressure of $7.14 \mathrm{MPa}$ (1050 psia). In this case, low-quality water is driven through the venturi as choking occurs. Figure D-6 shows a little poorer comparison of the MELCOR results with data particularly in the mid-range of the test when a distinct flow quality change in the entrained fluid shows up in the data. Several time-step sensitivity calculations were run with maximum time-step sizes of $1 \times 10^{-2} \mathrm{~s}$ and $0.50 \times 10^{-2} \mathrm{~s}$. The calculations revealed no particular time-step sensitivity for this problem.

MELCOR seems adequate in predicting most blowdown scenarios. For the intended purpose extreme accuracy is unnecessary and the results are generally satisfactory.

\section{D.4.2. Condensation}

To assess the condensation model in MELCOR a simple vertical test problem was set up. Seven control volumes were arranged vertically (see Annex D-2 at the end of this appendix). The first was run as a constant source of subcooled liquid with a $\Delta \mathrm{T}$ subcooling of approximately $22^{\circ} \mathrm{C}$. Each of the first six mesh cells was $1 \mathrm{~m}$ long with a $1-\mathrm{m}^{2}$ flow area and the cold liquid was injected at $1 \mathrm{~m} / \mathrm{s}$. A much bigger volume (CVH-70) of $100 \mathrm{~m}^{3}$ on the top provided a large source of steam. Steam with an initial superheat of approximately $7^{\circ} \mathrm{C}$ filled the tube and larger volume. Two cases simulated (1) the thermodynamic equilibrium option, and (2) the thermodynamic nonequilibrium option. Figure D-7 shows a schematic of the input.

Both cases show the classic difficulties associated with low-order finite-difference schemes in an Eulerian formulation. This includes pressure spikes when the void fraction of a mesh cell approaches 0.0 and stair steps rather than the more realistic continuous behavior. A certain amount of stair-stepping should also be evident in TRAC and RELAP for this type of problem.

The equilibrium case, ignoring these difficulties, appears to function as intended. Figures D-8 through D-13 show the void fraction, pressure, and vapor and liquid temperatures and velocities, respectively. However, for a realistic ECC injection case with high injection rates and large subcooling, equilibrium thermodynamics is not an accurate representation and causes grievous numerical difficulties.

Figures D-14 through D-19 show the results of the nonequilibrium case. It may be seen that the vapor velocities are never negative so that almost no condensation is taking place. This occurs because of the use of a very small interfacial area as the default value. Also note some initial diffusion of liquid into the second mesh cell (CVH-30) before the first cell fills up. This shows the filling of the first cell by about $0.50 \mathrm{~s}$ as shown in the void fraction or pressure plot (note the pressure blips that correspond to mesh cell filling).

The vapor velocities are for certain short times a little larger than the liquid velocities (peculiar in itself) but seem hardly enough to entrain liquid upward. The MELCOR staff suggested that if a smaller opening height is used, the entrainment problem will disappear. However, this again points out a basic problem with MELCOR; for vertical volumes, the opening height selected partially determines the void distribution. When liquid enters the large top tank, the condensation rate goes up by a relative factor of 100 and a noticeable pressure drop occurs.

A reasonable conclusion is that MELCOR probably cannot handle ECC injection problems accurately with the default intertacial area value. Although MELCOR is not really a reflood code, it could be expected that too much liquid and too much subcooling would enter the 


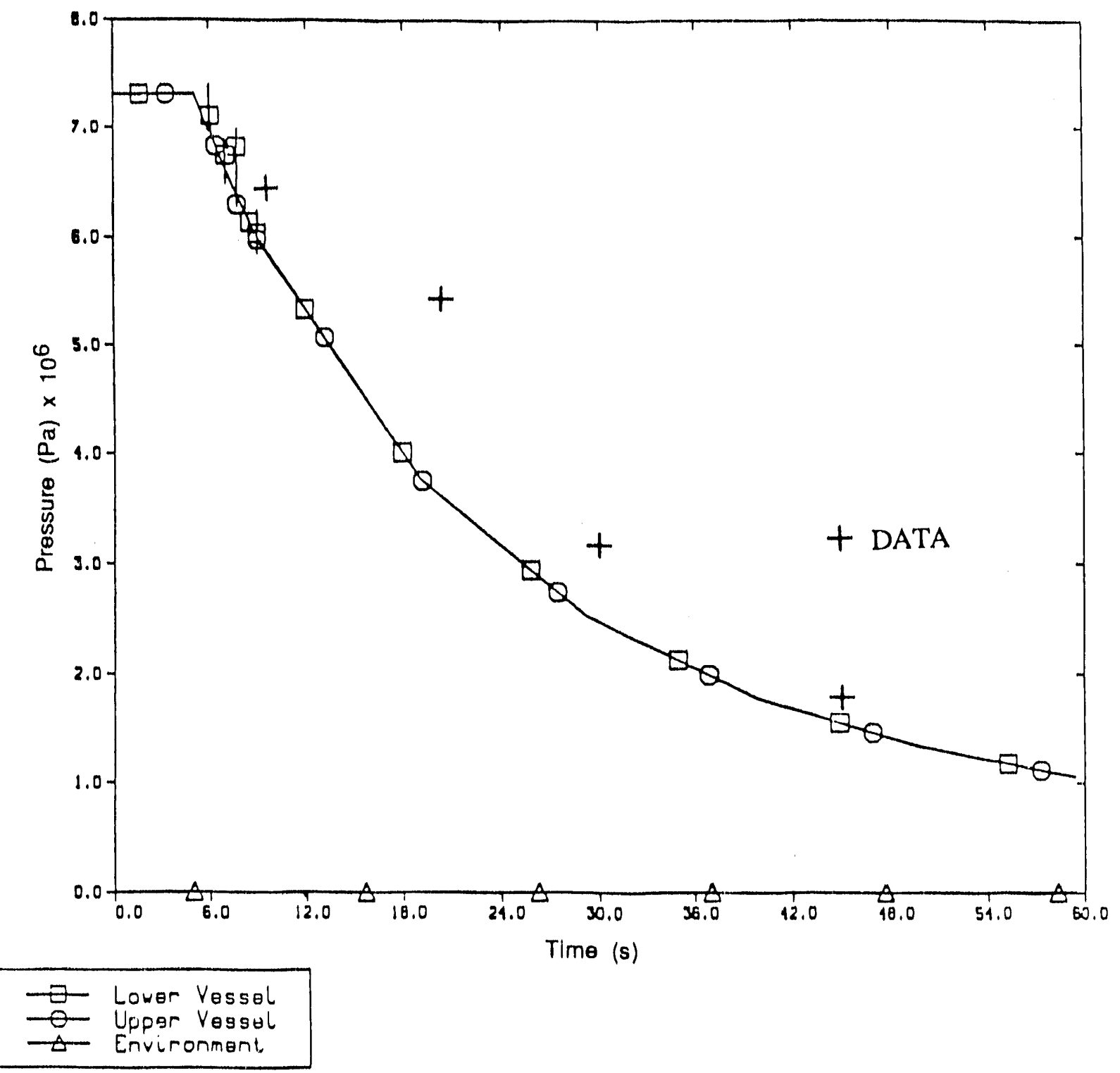

Fig. D-6.

Comparison of MELCOR pressure predictions and GE Test 5803-1 experimental data (choking factor of 1.0; maximum time step of $2 \mathrm{~s}$ ).

D- 14 


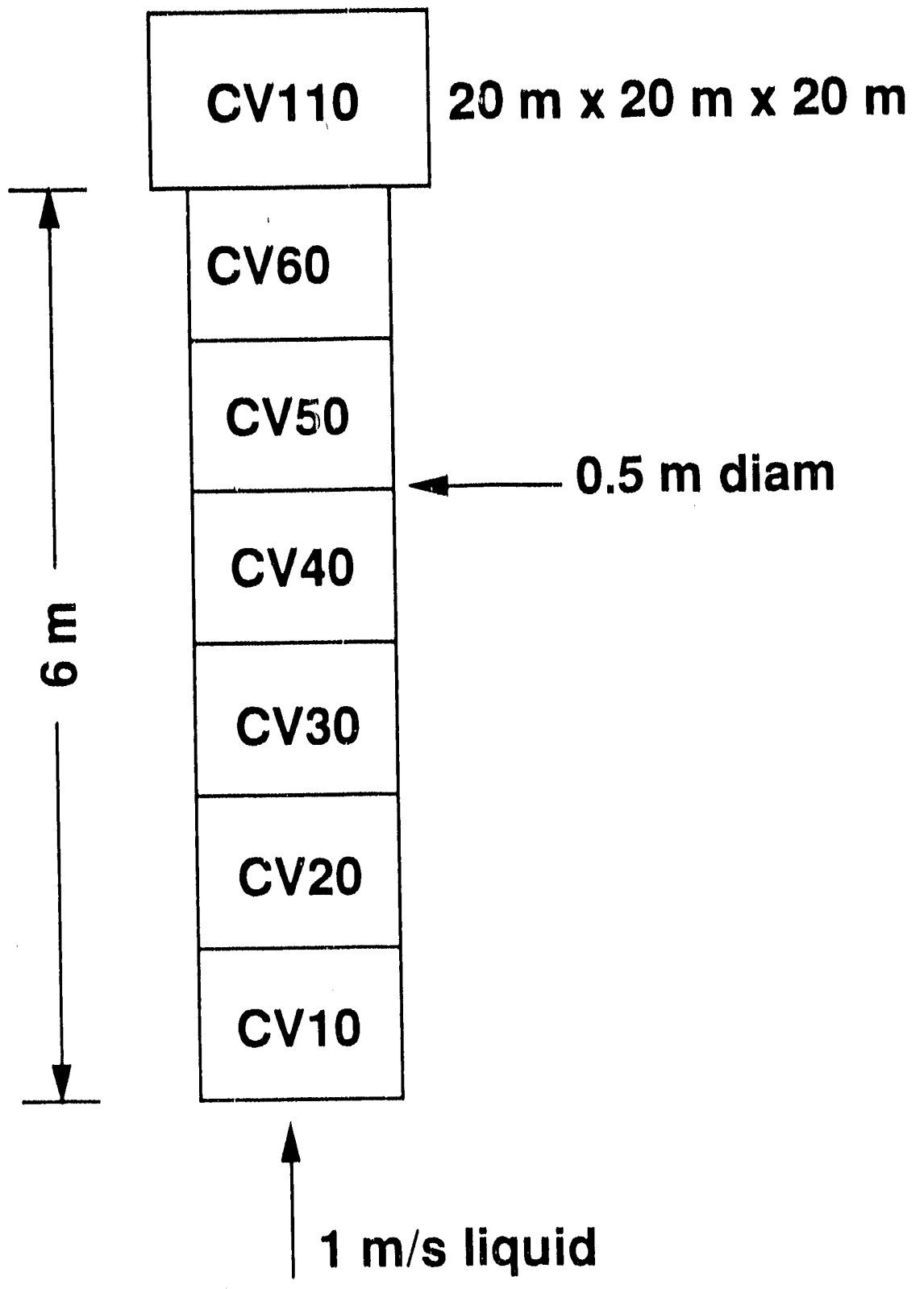

Fig. D-7.

Condensation probiem schematic. 


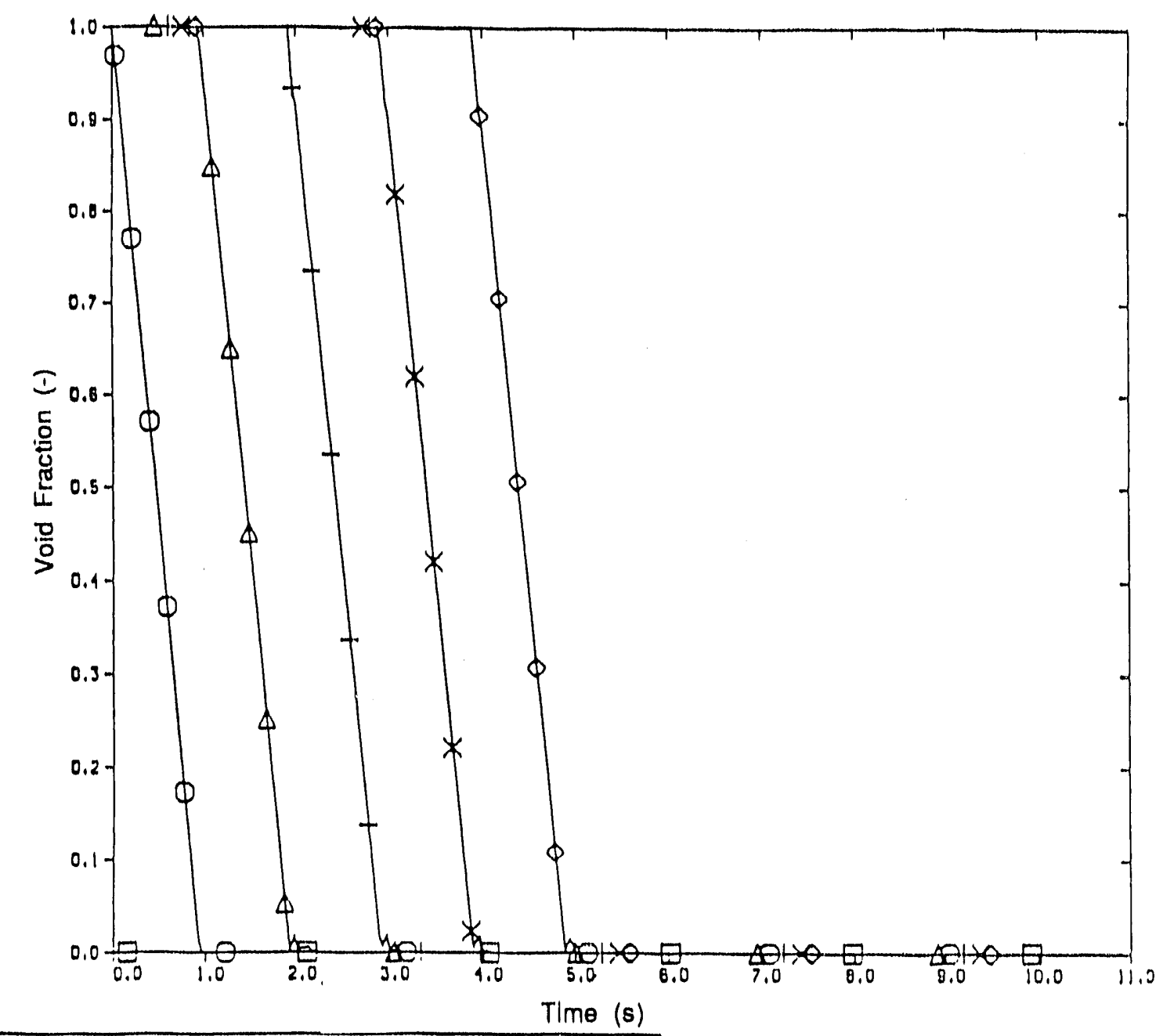

E evh-void.010 $\rightarrow$ evh-void.050.

O cuh-veid.020 a crhoroid.060

a crh-veid.030

+ crarroid.040

Fig, D-8,

Void fraction vs time (equilibrium thermodynamics; $1-\mathrm{m} / \mathrm{s}$ fill). 


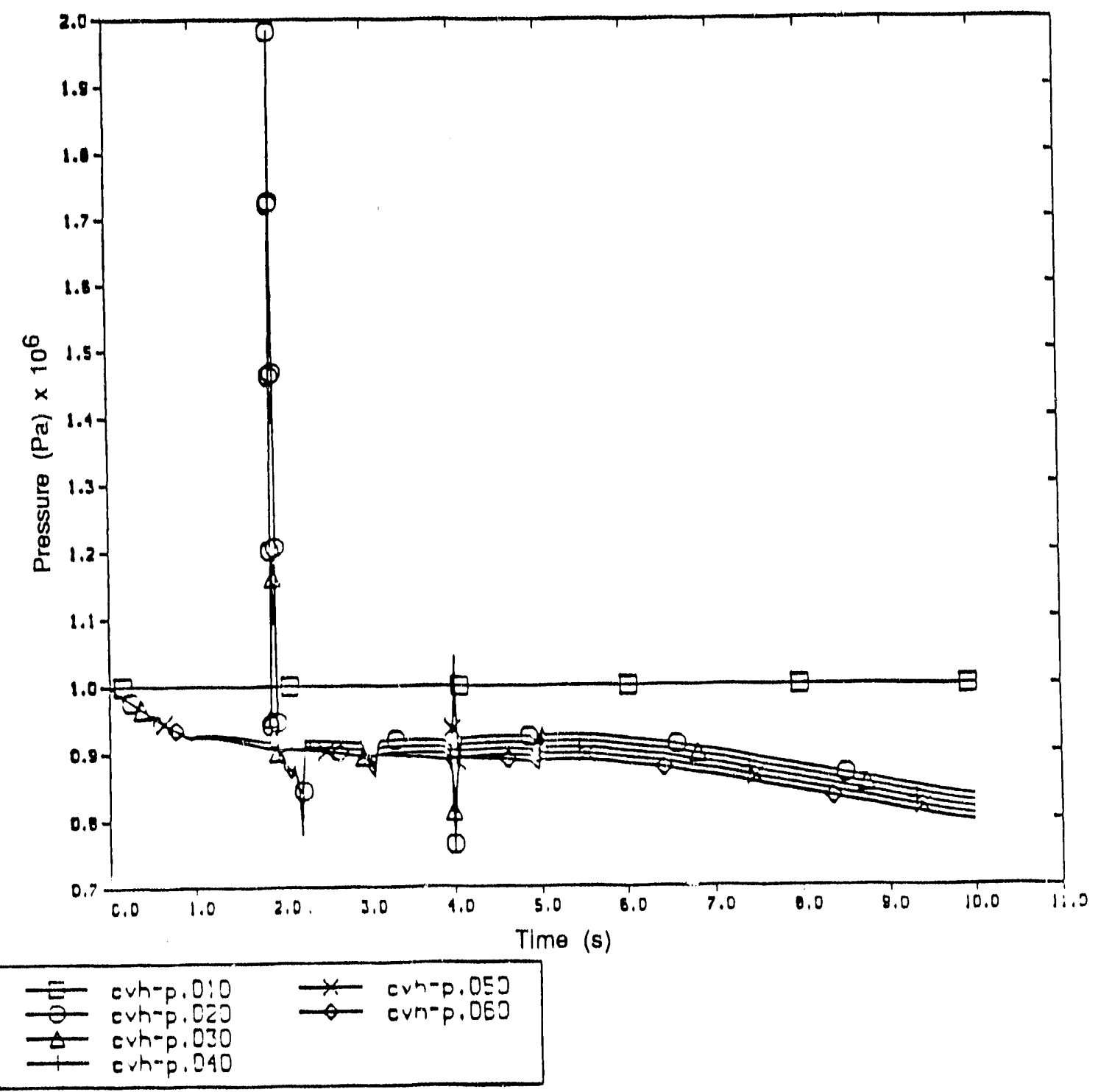

Fig. D.9.

Pressure vs time (equilibrium thermodynamics; $1-\mathrm{m} / \mathrm{s}$ fill).

D. 17 


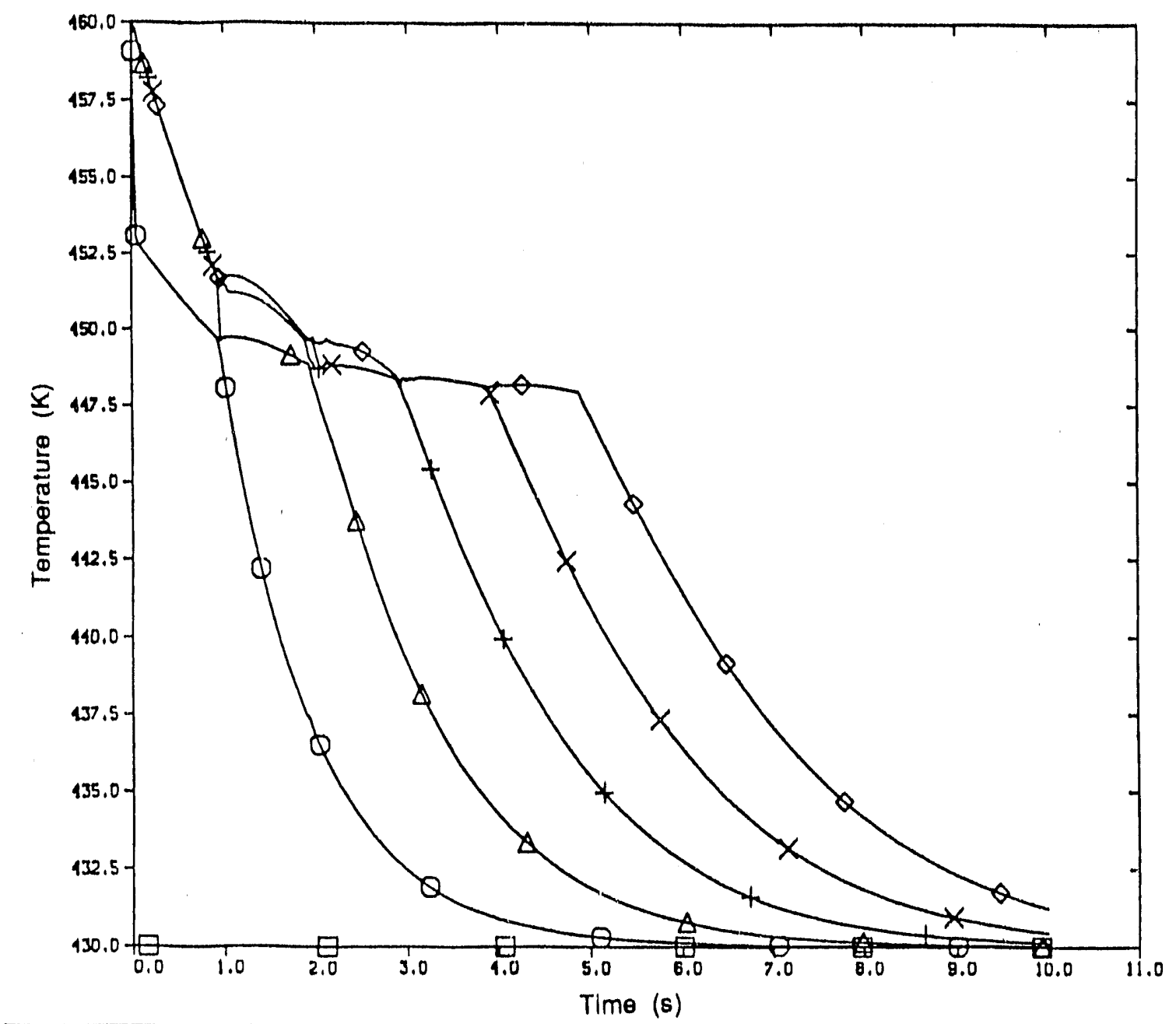

$$
\begin{aligned}
& \begin{array}{l}
\text { crh-trop.010 } \\
\text { croh-trap.020 }
\end{array} \text { crh-trap.050 } \\
& \text { covh-lvap.030 } \\
& \text { cruh-tvop.040 }
\end{aligned}
$$

Fig. D-10.

Vapor temperature vs time (equilibrium thermodynamics; $1-\mathrm{m} / \mathrm{s}$ fill). 


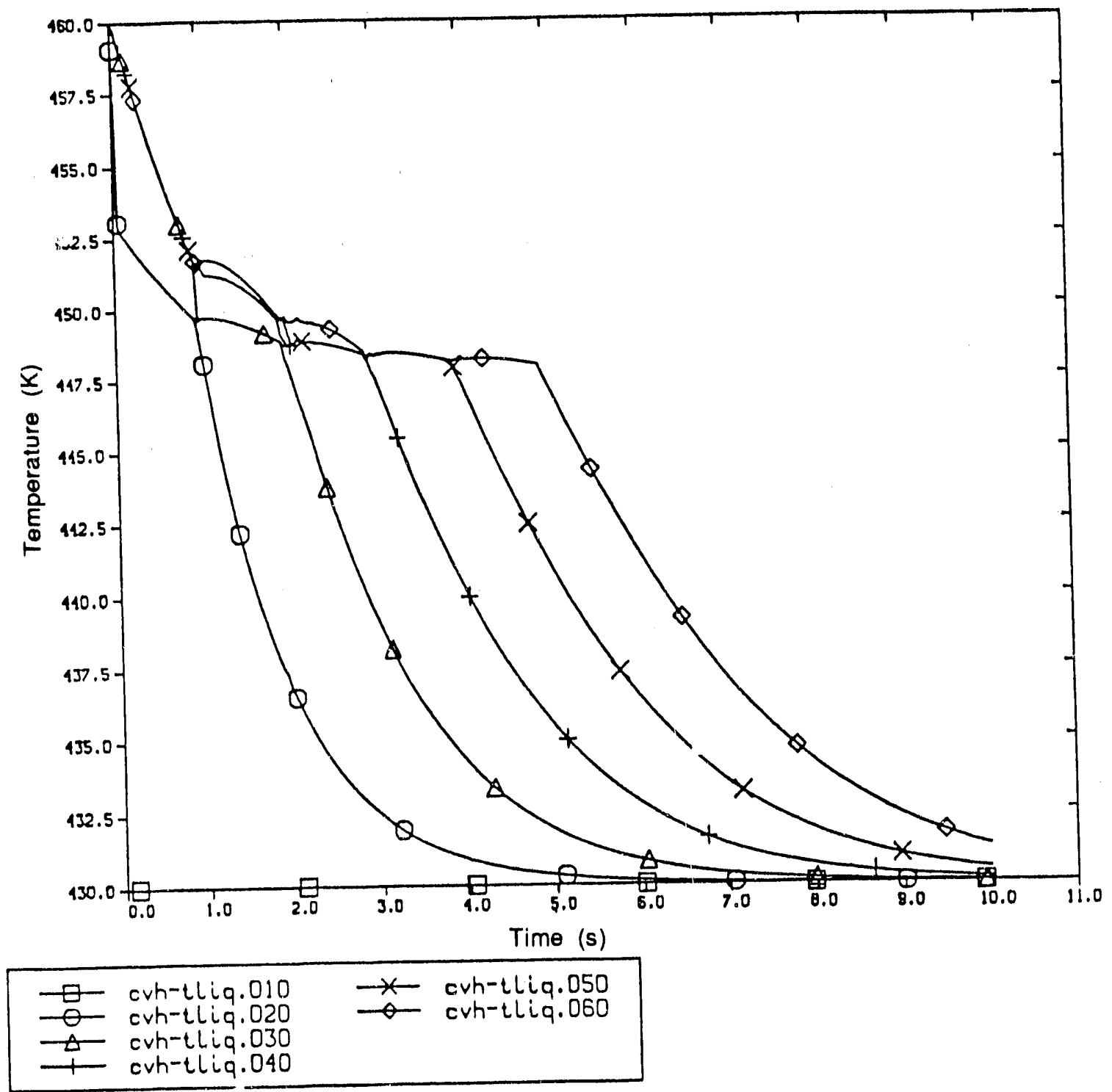

Fig. D-11.

Liquid temperature vs time (equilibrium thermodynamics; $1-\mathrm{m} / \mathrm{s}$ fill).

D-19 


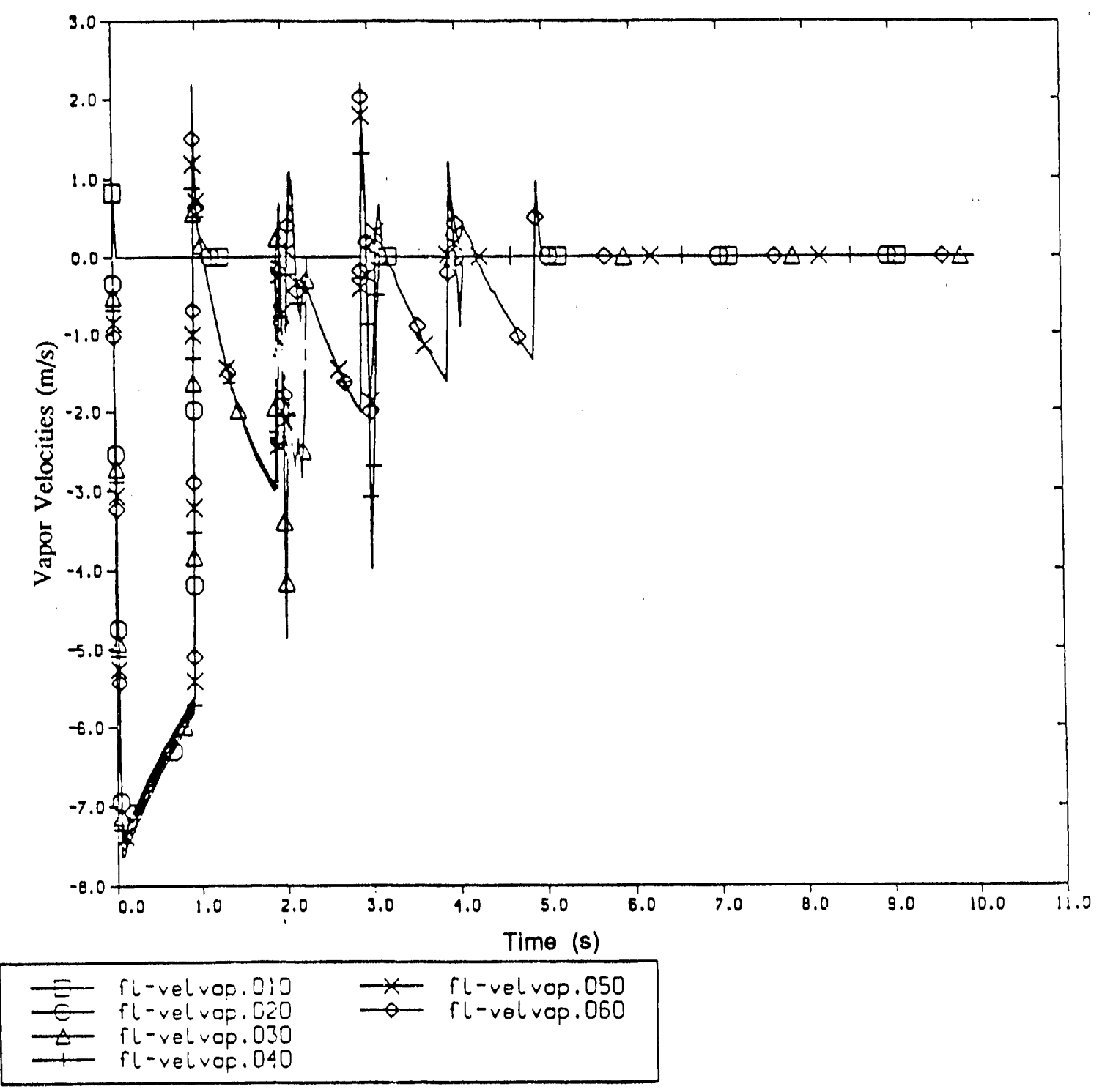

Fig. D-12.

Vapor velocity vs time (equilibrium thermodynamics; $1-\mathrm{m} / \mathrm{s}$ fill). 


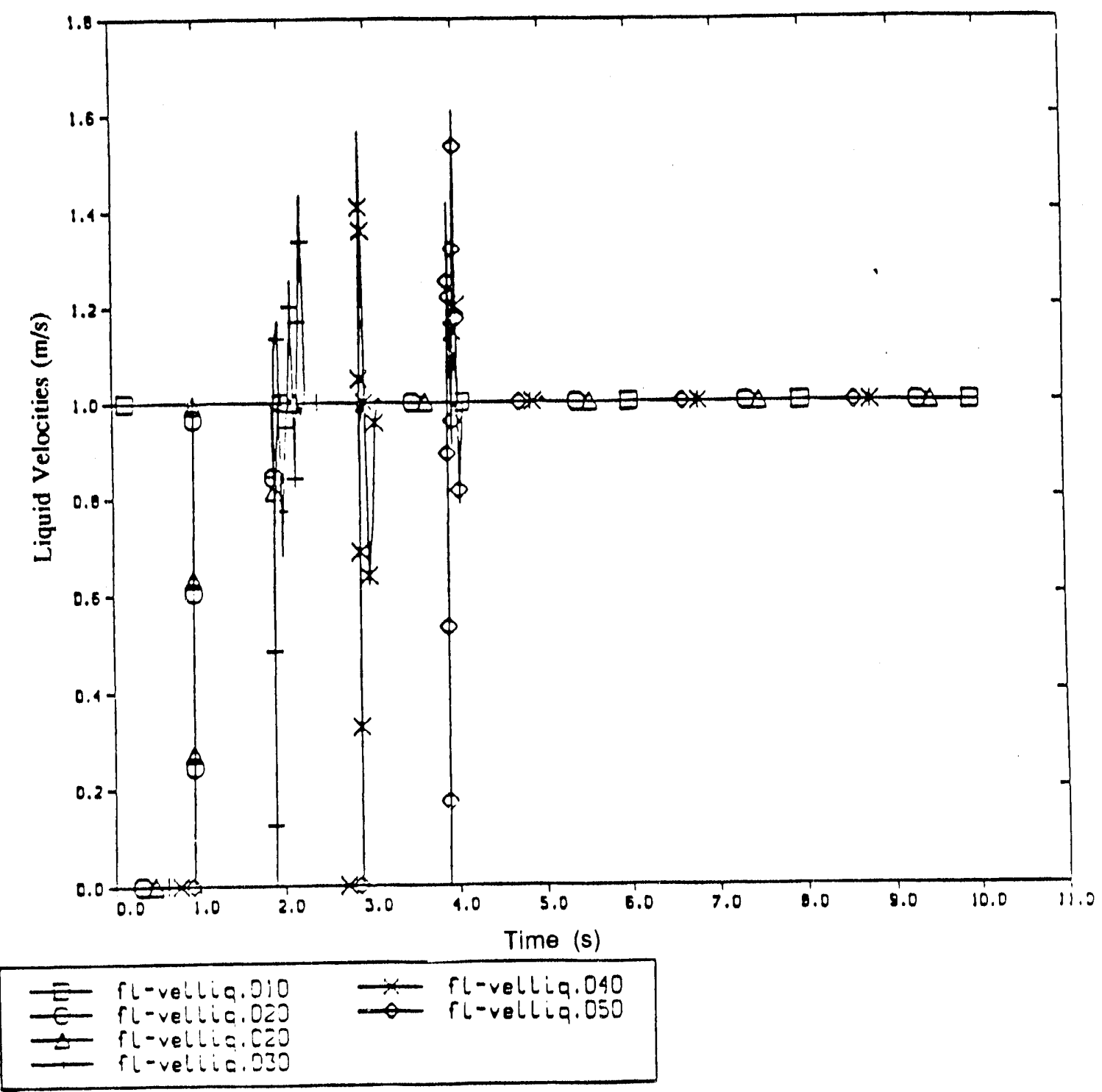

Fig. D-13.

Liquid velocity vs time (equilibrium thermodynamics; $1-\mathrm{m} / \mathrm{s}$ fill). 


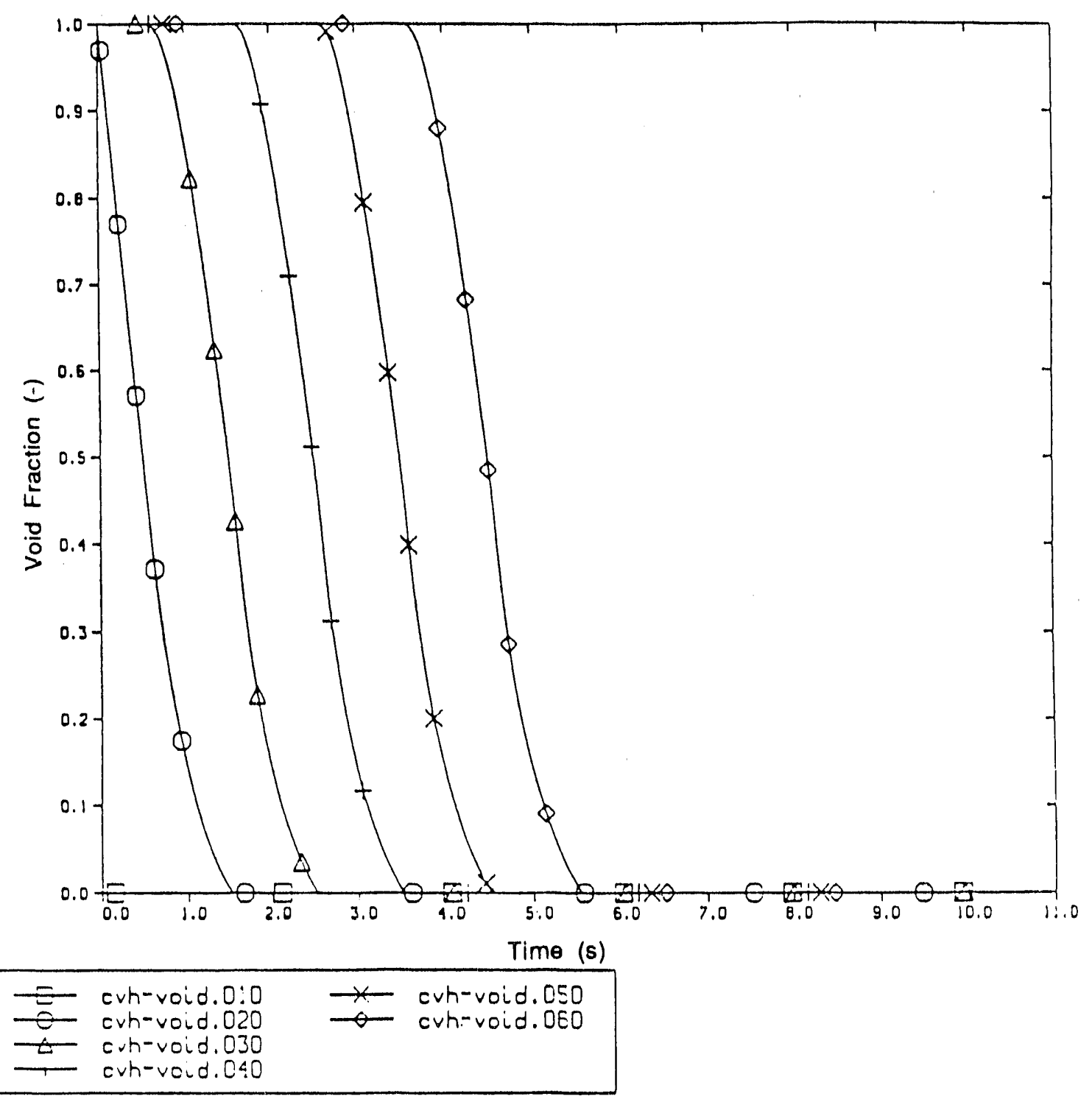

Fig. D-14.

Void fraction vs time (nonequilibrium thermodynamics; $1-\mathrm{m} / \mathrm{s}$ fill). 


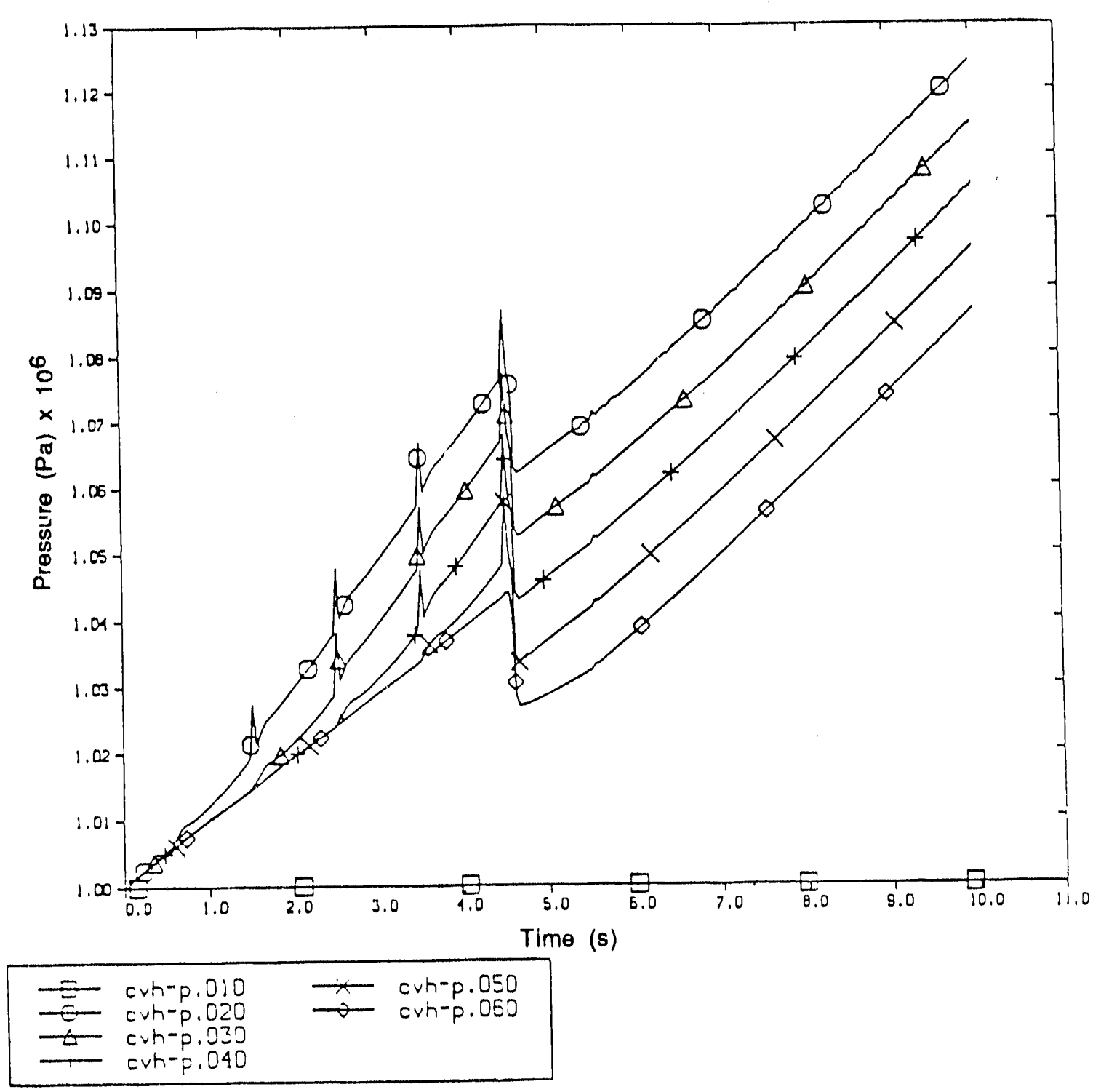

Fig. D-15.

Pressure vs time (nonequilibrium thermodynamics; $1-\mathrm{m} / \mathrm{s}$ fill).

D-23 


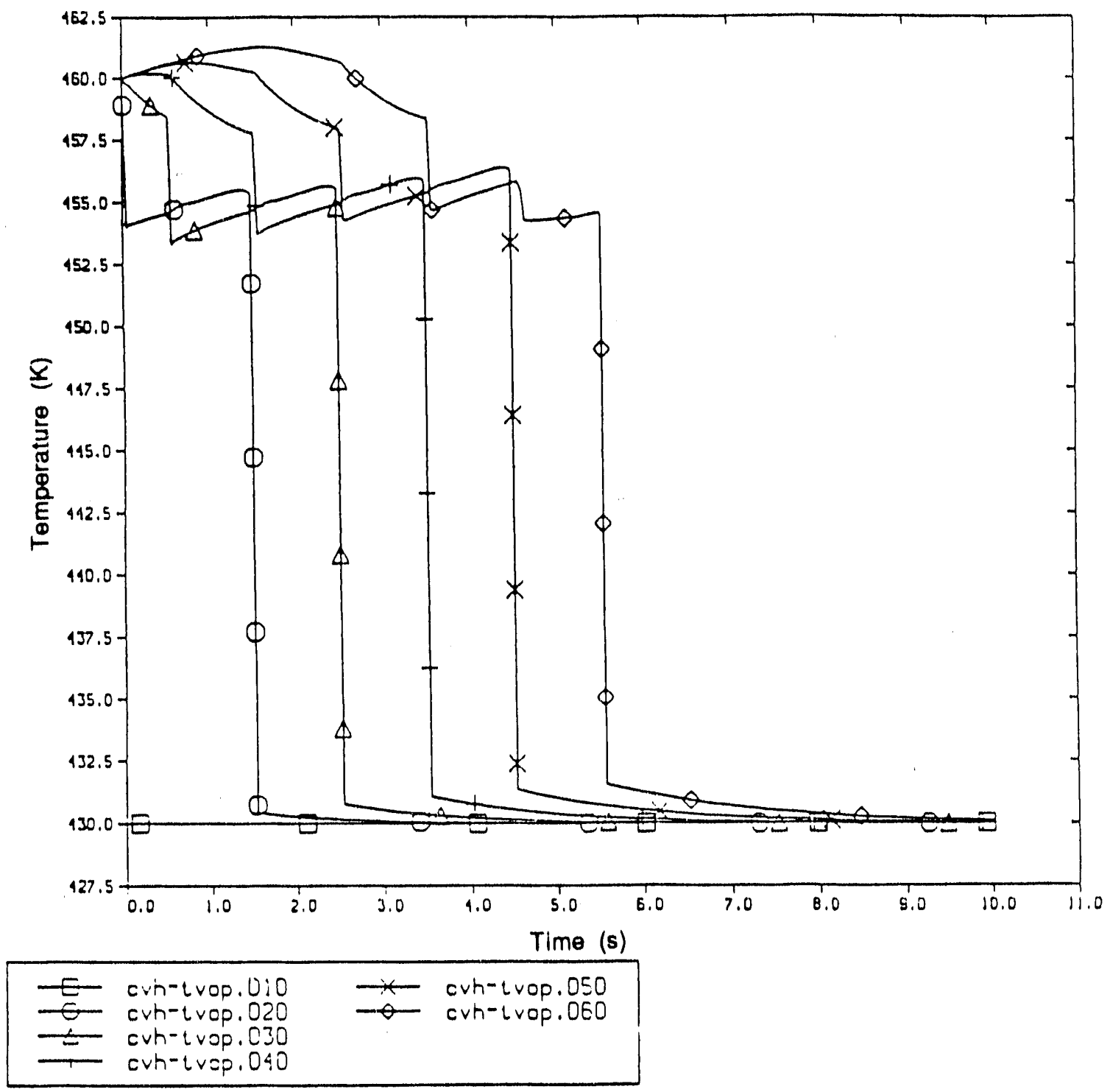

Fig. D-16.

Vapor temperature vs time (nonequilibrium thermodynamics; $1-\mathrm{m} / \mathrm{s}$ fill). 


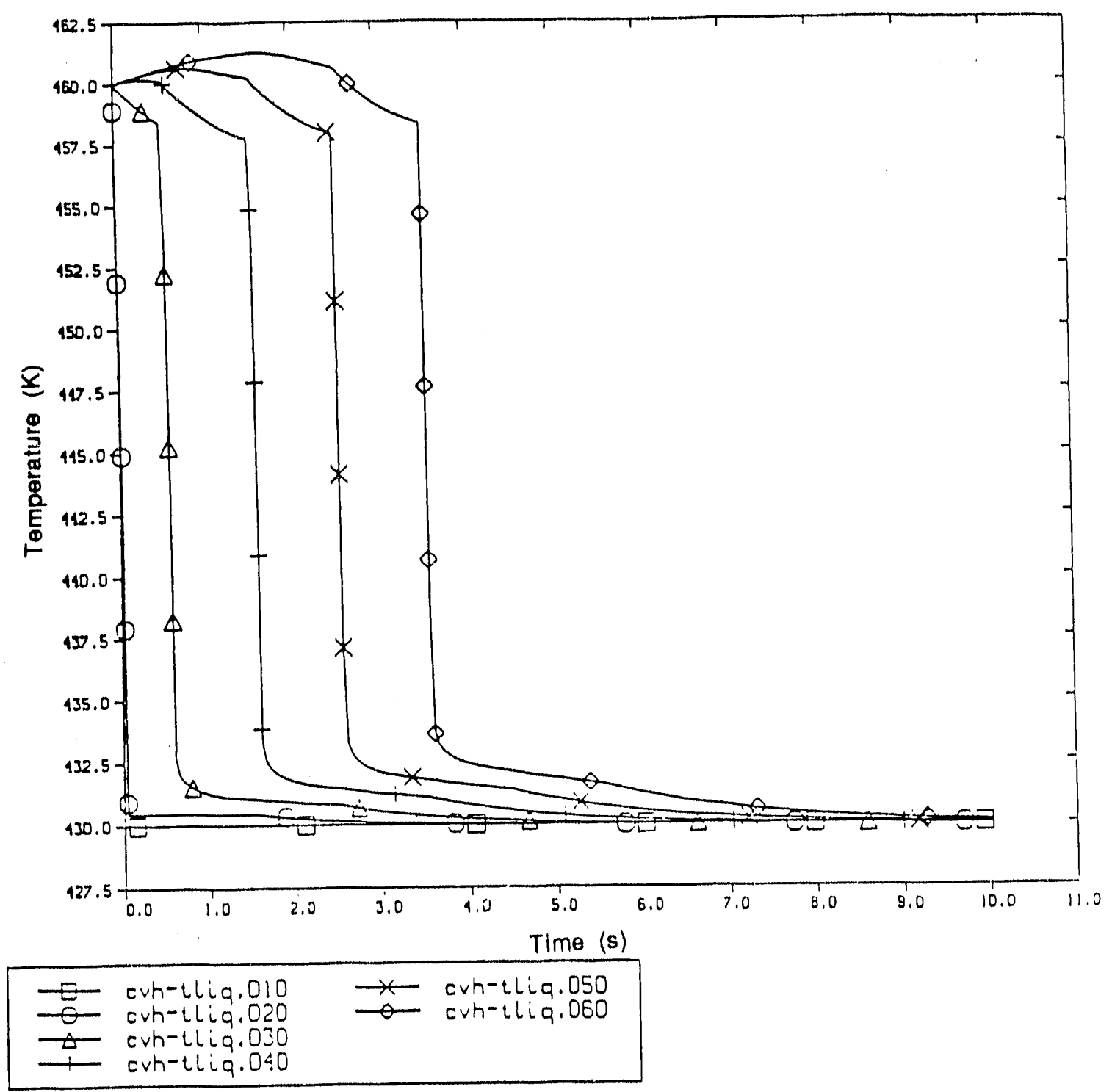

Fig. D-17.

Liquid temperature vs time (nonequilibrium thermodynamics; $1-\mathrm{m} / \mathrm{s}$ fill). 


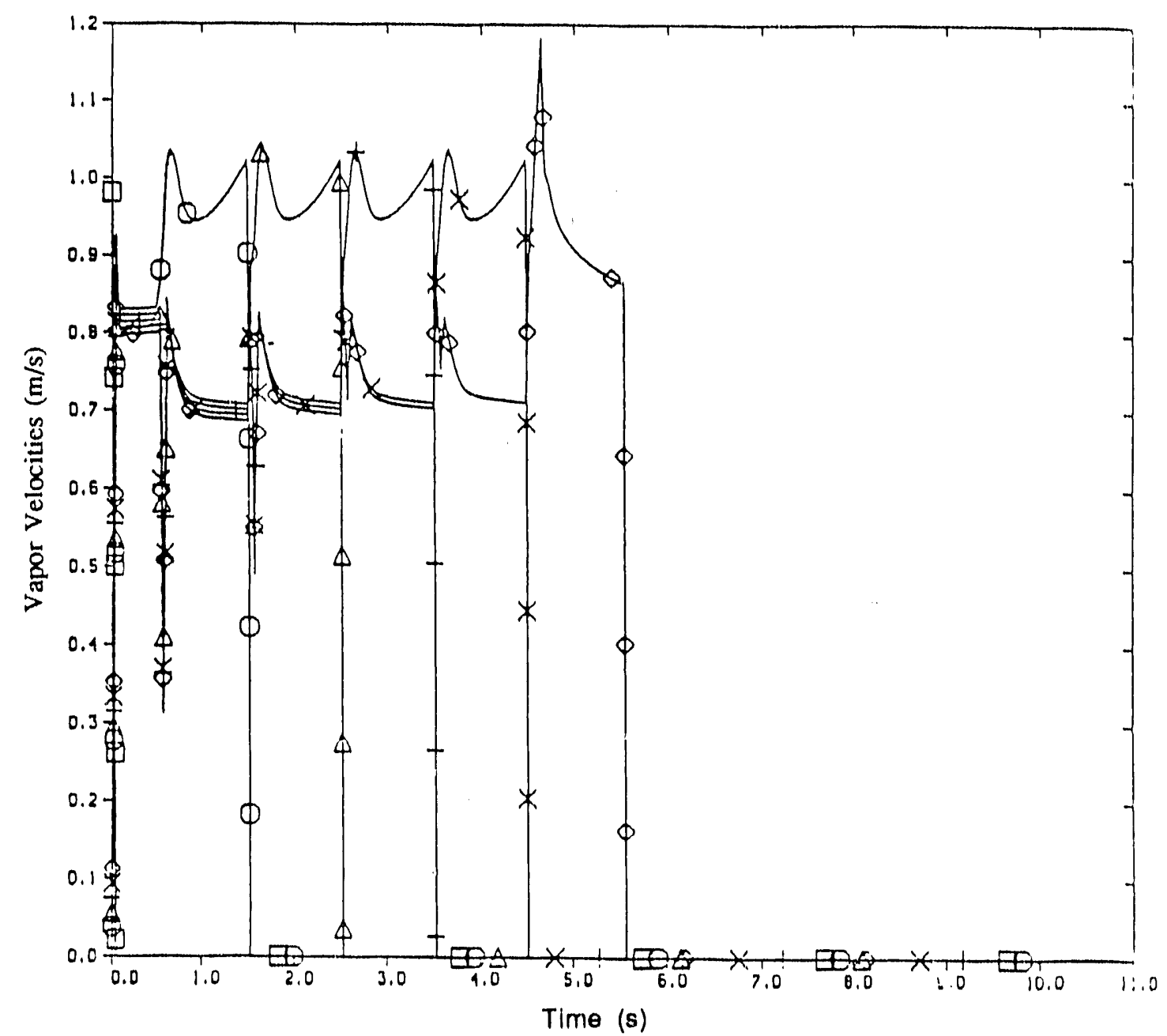

E rl-velvop.010

E- ri-velvap.0zo

$\longrightarrow$ fl-velvop.050

$\rightarrow$ rl-velvop.030

- fl-velvap.040

Fig. D-18.

Vapor velocity vs time (nonequilibrium thermodynami s; $1-\mathrm{m} / \mathrm{s}$ fill). 


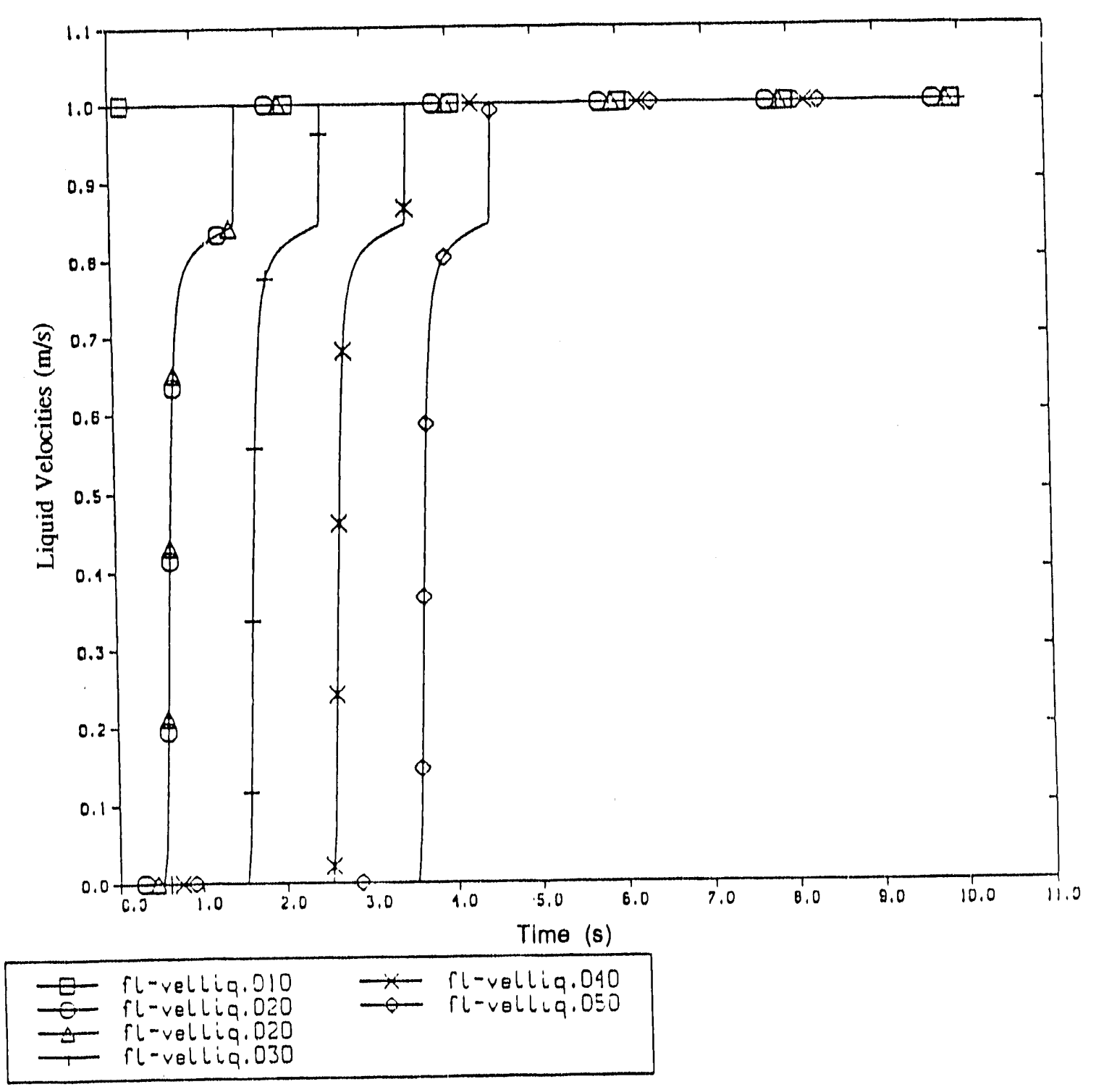

Fig. D-19.

Liquid velocity vs time (nonequilibrium thermodynamics; $1-\mathrm{m} / \mathrm{s}$ fill). 
lower plenum unless the interfacial rates are increased by a factor of greater than 10. Because condensation is a flow-regime-specific phenomena, a single value of the augmentation factor is inappropriate.

A separate problem associated with the MELCOR condensation model, discussed earlier, is the potential of overprediction in condensation rates for conditions when a steam atmosphere occupies a large quiescent water pool.

\section{D.4.3. Air-Water Closed Loop}

Ten vertical control volumes were stacked with a much larger tank on the top. All of the lower 10 cells (CV10 - CV100) were $0.5 \mathrm{~m}$ in diameter and $1 \mathrm{~m}$ high. The final control volume CV110 was $20 \times 20 \times 20 \mathrm{~m}$. Figure D-20 is a schematic of the model. Because an opening height was needed to connect vertical mesh cells, the diameter of $0.5 \mathrm{~m}$ was chosen. A fan (momentum source) set for a velocity of $10 \mathrm{~m} / \mathrm{s}$ and a flow link connected CV110 with the lowest pipe cell CV10 to form a closed loop. The initial condition had half of the 10-cell pipe filled completely with water before the fan was activated. The upper five mesh cells and the tank were filled with air.

Figures D-21 through D-26 show the final void distribution for several voiames. Each cell settles out to a uniform void fraction of 0.5 . This is entirely due to the pool/atmosphere concept of a flow regime coupled with an opening height equal to the mesh cell height divided by' 2. In other words, the opening height for this problem uniquely determines the void distribution. This is a recurring problem with MELCOR and can only be addressed by designating the full vertical height as an opening height, using only one rather than multiple flow links as connectors, and developing a more detailed flow map and interfacial drag package and incorporating them into the code.

\section{D.4.4. Summary}

The experimental basis for CVH/FL is currently very limited, especially when dealing with thermal-hydraulic regimes typical of the RCS geometry (BWRs and PWRs) and under severe accident conditions. Additional separate-effects benchmarking studies are needed to identify problems, pitfalls, and features of the CVH/FL package.

\section{D.5. IDENTIFIED DEFICIENCIES AND PLANNED IMPROVEMENTS}

MELCOR improvement needs have also been assessed and documented by the MELCOR development staff at Sandia (Ref. D-18). The identified deficiencies and planned improvements that are relevant to the $\mathrm{CVH} / \mathrm{FL}$ packages include the following.

(1) Numerics and time-step control sensitivity

- Improved coupling between the $\mathrm{CVH} / \mathrm{FL}$ and the $\mathrm{RN}$ packages

- Implementation of a method for valves on a CVH subcycle basis

(2) Modeling improvement

- Development of an improved interfacial momentum exchange model

- Development of improved models for simulation of natural circulation flows in PWRS 


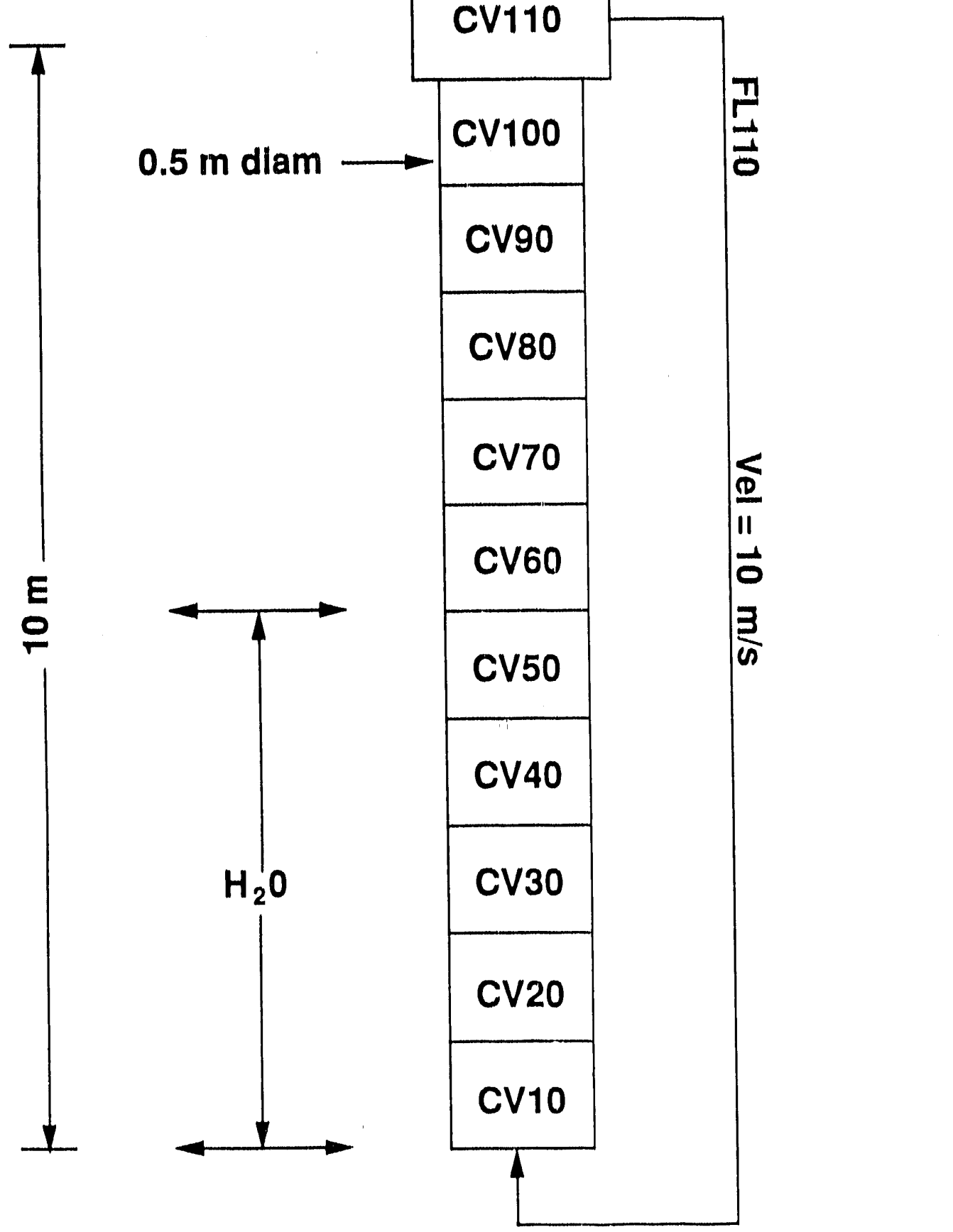

Fig. D-20.

Condensation problem schematic. 


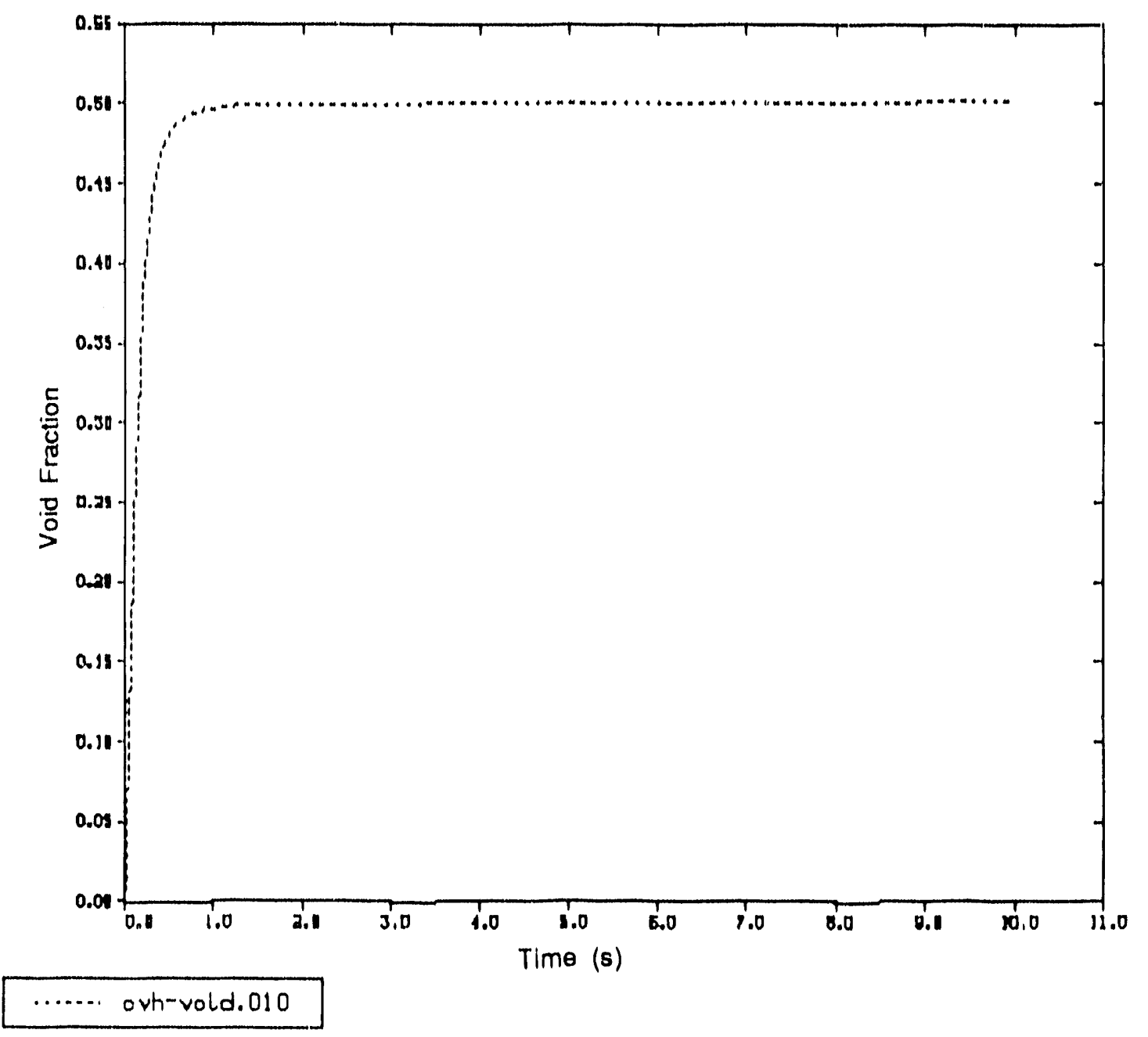

Fig. D-21.

Air-water closed loop-void fraction vs time (cell 10).

D-30 


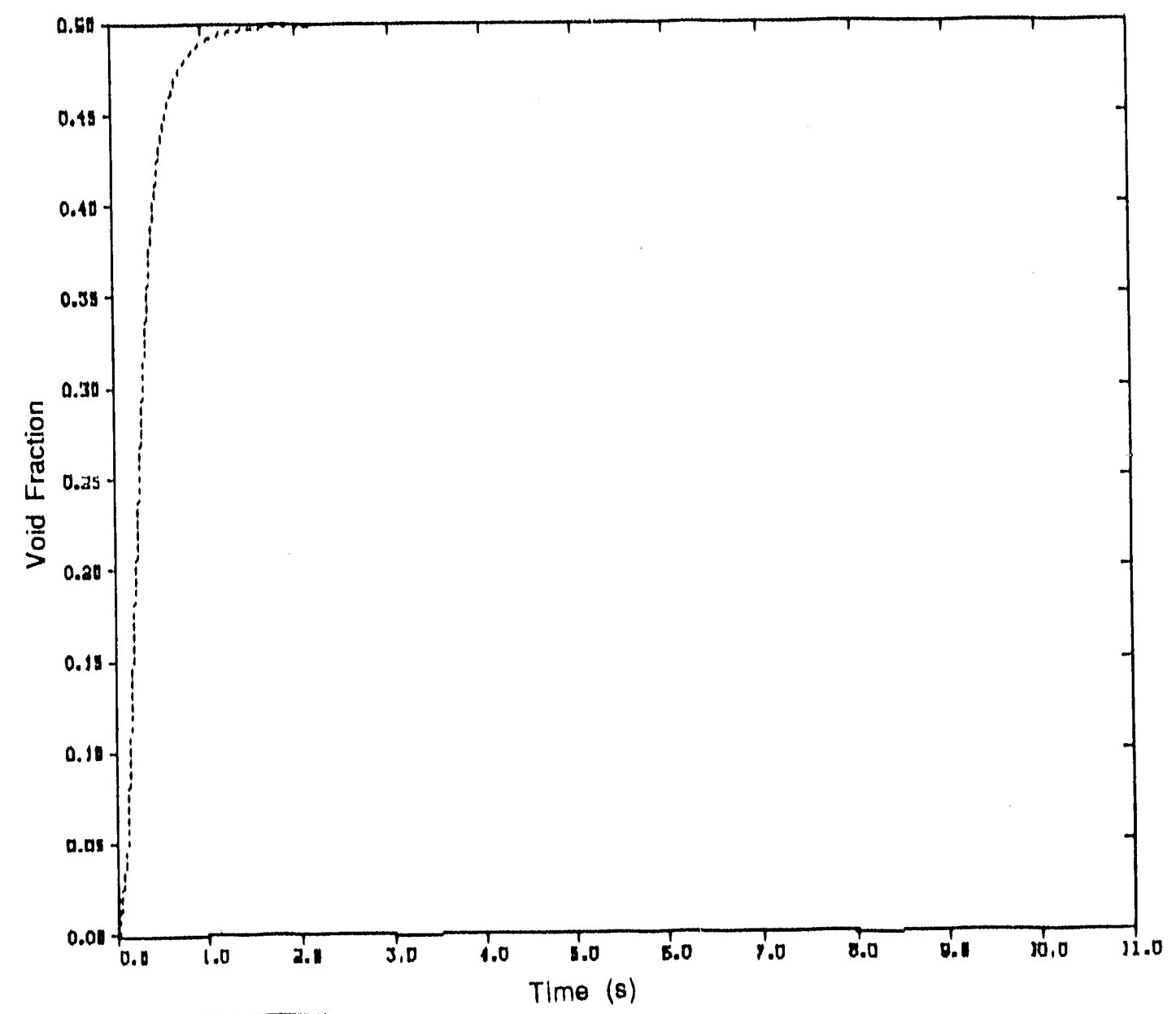

....... ovh-void.030

Fig. D-22.

Air-water closed loop-void fraction vs time (cell 30).

D-31 


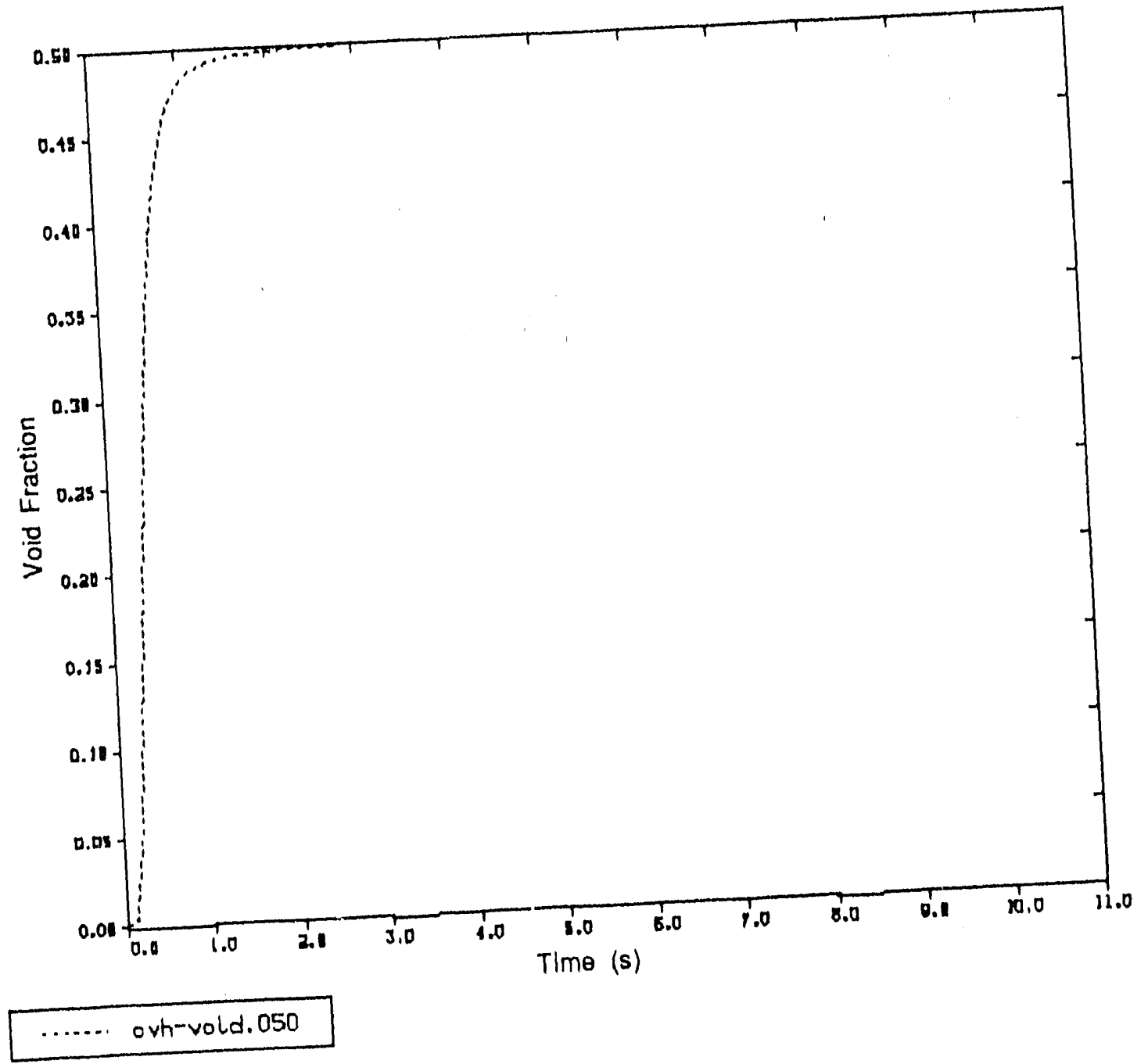

Fig. D-23.
Air-water closed loop-void fraction vs time (cell 50).

D-32 


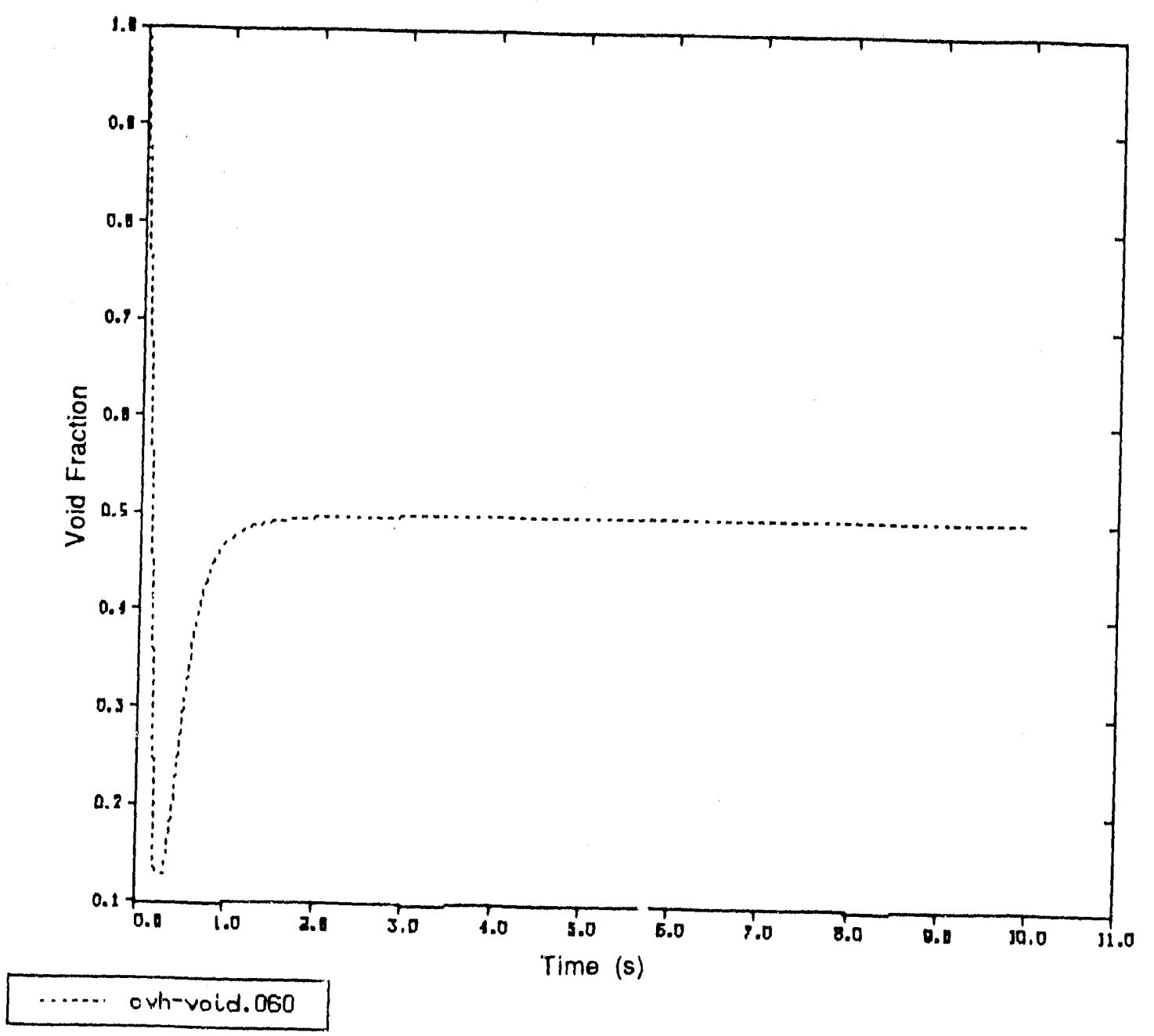

Fig. D-24.

Air-water closed loop-void fraction vs time (cell 60).

D-33 


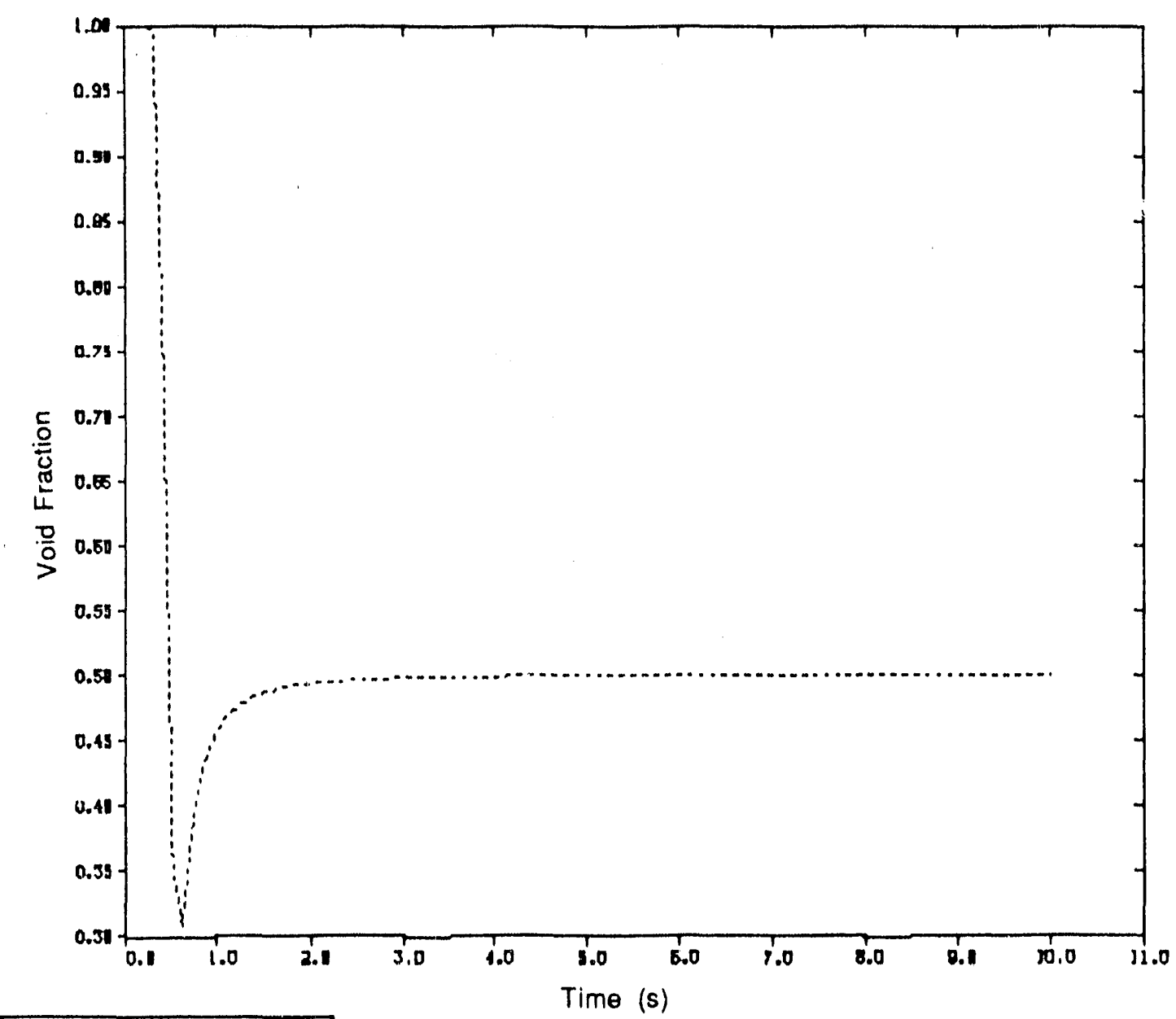

...... orh-vold. 080

Fig. D-25.

Air-water closed loop-void fraction vs time (cell 80).

D-34 


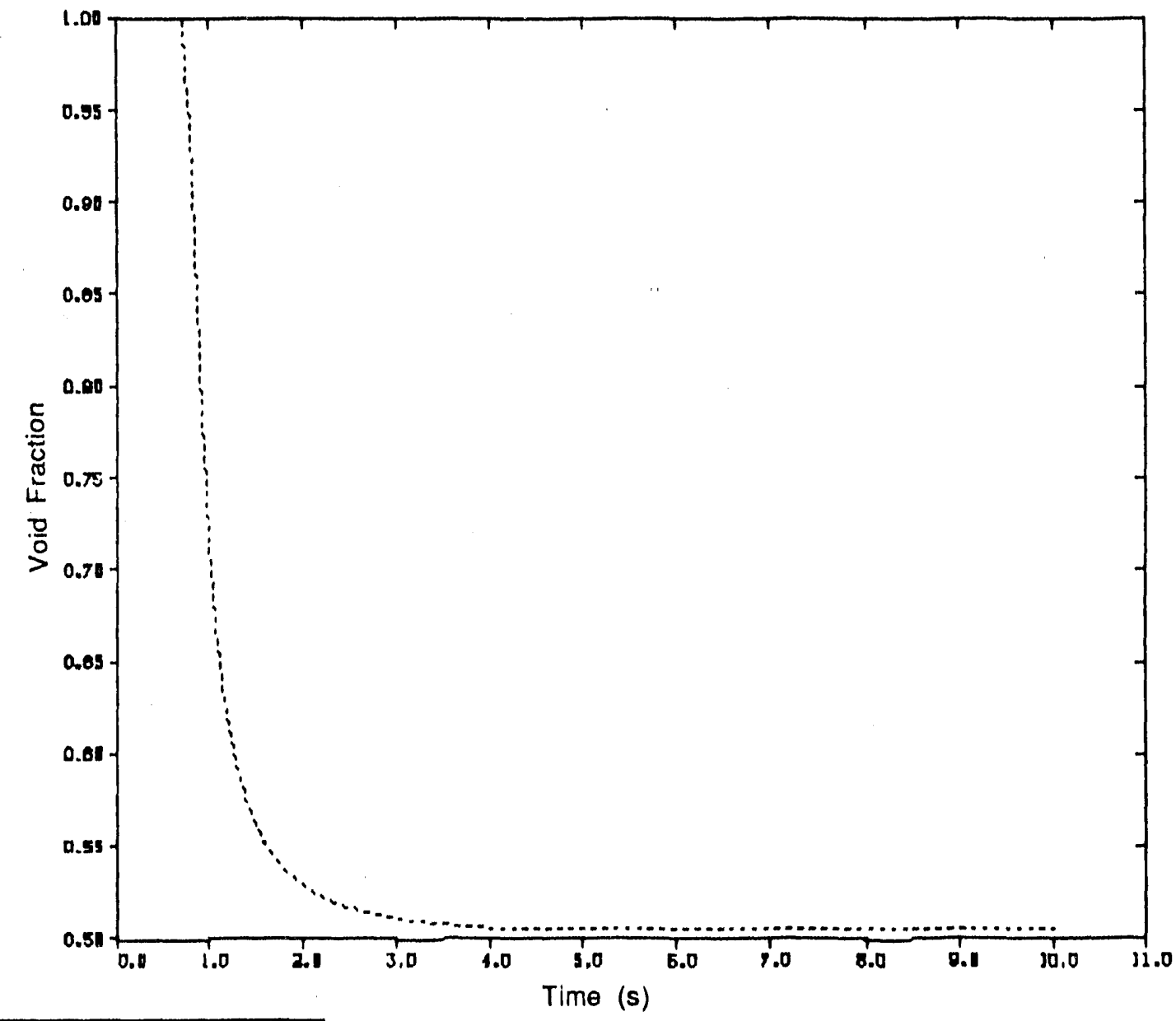

....... ovh-vold. 100

Fig. D-26.

Air-water closed loop_void fraction vs time (cell 100). 


\section{D.6. SUMMARY FINDINGS}

\section{D.6.1. Review Findings and Relationship to Dominant Phenomena}

Table D-Il summarizes the review findings in relation to the key severe accident phenomena described in Appendix C. These findings are stated in relation to the dominant invessel and ex-vessel phenomena for the following time intervals:

(a) Initial transient, depletion, and heatup interval (before core damage)

(b) Core uncovery interval (start of core damage)

(c) Melt relocation and slump interval (substantial core damage)

(d) Core debris material inside the lower plenum interval (late in-vessel phase)

(e) Containment and post-vessel failure interval

Table D-III lists the categorization of the review findings for technical adequacy, using the guidelines discussed in Chapter 1 . For each aforementioned time interval, the various $\mathrm{CVH} / \mathrm{FL}$ models are categorized by evaluating the following key attributes set forth by the categorization guidelines of Chapter 1:

(a) Adequacy of Physics: This addresses the acceptability of the model physics as formulated in the CVH/FL packages.

(b) Adequacy of the Numerical Methods: This addresses the acceptability of the numerics as implemented in the $\mathrm{CVH} / \mathrm{FL}$ packages.

(c) State of Knowledge: This addresses the strengths of the available knowledge and/or data base (strong, good or poor, and uncertain).

(d) Importance of Phenomena: This addresses the importance of given process/phenomenon to prediction of accident progression in the time frame under evaluation. The importance of a phenomenon may change from one time interval to another.

\section{D.6.2. Summary and Conclusions}

The basic hydrodynamics methods embodied within CVH/FL package are closer in principle to those of RELAP4 than TRAC or RELAP5 codes. The CVH/FL models are an adaptation of the approach employed by HECTR and CONTAIN containment analysis codes.

The generalized network architecture, volume/altitude, and virtual volume approach of $\mathrm{CVH} / \mathrm{FL}$ provide a valuable tool for application of MELCOR to variety of nuclear reactor designs.

A prioritized summary list of Categories 4 and 5 as well as other important review findings is provided in Table D-IV. The current model does not inciude provisions for simulation of buoyancy-induced recirculation flows within the core (PWRs), upper plenum, and countercurrent (all liquid or all vapor) single-phase flow fields typical of those expected during low-flow natural convection conditions within the hot-leg region of PWRs for severe accidents at high RCS pressures.

There is insufficient experimental benchmarking of the $\mathrm{CVH} / \mathrm{FL}$ packages. The ultimate judgment for determination of the adequacy of hydrodynamic models requires more extensive comparisons with both separate-effects and integral tests. 
The inherent limitations of the well-mixed control volume approach in simulation of truly multidimensional phenomena need to be made very clear as part of the CVH/FL documentation and manuals.

Potential issues impacting CVH/FL predictions include the following.

- Numerical approximations, averaging schemes, and absence of spatial derivative flux terms of the momentum equations, together with "loose" coupling between the CVH/FL and other MELCOR phenomenological packages can exasperate the MELCOR numerical problems reported by several users.

- Difficulties in defining control volume velocities for use in closure relations, when several flow paths are connected to any control volume.

- Prediction of stratification (with and without noncondensables) using the $\mathrm{CVH} / \mathrm{FL}$ requires sufficiently detailed nodalization and some knowledge of expected flow patterns.

- Countercurrent flow patterns within volumes cannot be modeled, especially for volumes with large aspect ratios (e.g., long pipes).

- Modeling of transport effects in pipe-like geometries is susceptible to numerical diffusion. This is typical of most computer codes that use similar methods.

- It is difficult and sometimes meaningless to define characteristic dimensions, loss coefficients, etc., when dividing a large, unobstructed volume into several interconnected control volumes and flow paths.

- In the present CVH/FL formulation, the junction opening heights determine what flows through a flow path. Specification of junction opening heights is subject to some interpretation; therefore, additional guidance is required for MELCOR users to avoid potential misinterpretations.

- Impact of blockage formation within the core region following core degradation on hydrodynamic calculations can only be partially treated (flow area changes through the CFs/TFs). This can strongly impact in-vessel flow circulation, hydrogen generation, and core melt progression.

- The present pool/atmosphere condensation model is incorrect and needs to be corrected. Overprediction in condensation rates are expected for conditions such as a steam atmosphere overlying a large quiescent water pool.

- The present approach to momentum transfer between pool and atmosphere phases within flow paths needs further assessment. This model is inadequate for prediction of pressurizer drainage under PWR severe accident conditions.

- Specific models for pressurizer sprays, BWR core sprays, and flows through ice beds are not currently available within CVH/FL or other MELCOR phenomenological packages.

- MELCOR cannot model reflood properly. Application of MELCOR to evaluate PRA success criteria and accident management strategies is questionable. 
Table D-IIa

Summary of Review Findings

(Initial Transient, Depletion, and Heatup Interval)

\begin{tabular}{|c|c|}
\hline Dominant Phenomena & Review Findings \\
\hline \multicolumn{2}{|l|}{ In-Vessel Phase } \\
\hline Discharge and blowdown & CVH/FL model appears reasonable. \\
\hline $\begin{array}{l}\text { Evaporation and condensation processes } \\
\text { (pool/atmosphere) }\end{array}$ & $\begin{array}{l}\text { Condensation model is not always correct. } \\
\text { Potential problems exist for large aspect ratio } \\
\text { geometries. Also, the experimental basis and range } \\
\text { of empirical correlations need justification. }\end{array}$ \\
\hline $\begin{array}{l}\text { Interface to heat structures for condensation } \\
\text { and evaporation }\end{array}$ & $\begin{array}{l}\text { Weak numerical coupling of } \mathrm{CVH} \text { and HS modules } \\
\text { can lead to cycling and numerical instabilities. } \\
\text { Detailed knowledge of incondensable gas } \\
\text { distribution is a must for accurate prediction of } \\
\text { evaporation and condensation processes. This can } \\
\text { also impact aerosol deposition on structural } \\
\text { surfaces. }\end{array}$ \\
\hline $\begin{array}{l}\text { BWR core and PWR pressurizer sprays } \\
\text { (ECCS effects) }\end{array}$ & $\begin{array}{l}\text { At present no models available. Some usage of the } \\
\text { CF/TF features possible. }\end{array}$ \\
\hline Pressurizer drainage (PWRs) & $\begin{array}{l}\text { No specific model exists. The present interfacial } \\
\text { exchange model is not adequate. }\end{array}$ \\
\hline
\end{tabular}


Table D-IIb

Summary of Detailed Findings (Core Uncovery Interval)

\begin{tabular}{|c|c|}
\hline Dominant Phenomena & Review Findings \\
\hline \multicolumn{2}{|l|}{ In-Vessel Phase } \\
\hline $\begin{array}{l}\text { Transport and effect of non- } \\
\text { condensable gases }\end{array}$ & $\begin{array}{l}\text { Inherent perfect mixing approach of CVH/FL requires (as } \\
\text { a minimum) extensive nodalization to predict "pocketing" } \\
\text { phenomena of hydrogen stratification in RCS geometry. } \\
\text { Perfect mixing would also produce "false diffusion" of } \\
\text { noncondensable gases within control volumes. Under } \\
\text { most severe accident conditions, perfect mixing is } \\
\text { probably adequate. However, depending on plant and } \\
\text { accidents, the CVH/FL approach may become less reliable. } \\
\text { This is a general problem with all control volume codes. }\end{array}$ \\
\hline & $\begin{array}{l}\text { Presence of hydrogen in upper portions of steam generator } \\
\text { U-tubes and upper plenum or reactor vessel can strongly } \\
\text { impact flow circulation and heat-transfer rates to structural } \\
\text { surfaces. }\end{array}$ \\
\hline & This phenomenon is less important for BWRs. \\
\hline $\begin{array}{l}\text { Natural circulation (in-vessel, } \\
\text { upper plenum, loops, steam } \\
\text { generators) }\end{array}$ & $\begin{array}{l}\text { Present model is inadequate because of assumptions of } \\
\text { perfect mixing, lack of models for re-entrant flows into the } \\
\text { core, problems with the so-called "dT/dZ" model of the } \\
\text { COR package, lack of models for cross flow in PWRs, } \\
\text { countercurrent flow in hot legs, and fluid mixing within } \\
\text { steam generator plena. }\end{array}$ \\
\hline & $\begin{array}{l}\text { This issue is of limited importance to BWRs (only after } \\
\text { seal clearing in the downcomers and within the upper } \\
\text { plenum area). }\end{array}$ \\
\hline $\begin{array}{l}\text { Condensation and evaporation } \\
\text { effects }\end{array}$ & Same as previous interval. \\
\hline Reflood & $\begin{array}{l}\text { MELCOR cannot model reflood properly. Application of } \\
\text { MELCOR for evaluation of PRA success criteria is } \\
\text { questionable. }\end{array}$ \\
\hline $\begin{array}{l}\text { Mixing and transport of } \\
\text { noncondensables }\end{array}$ & Same as above. \\
\hline $\begin{array}{l}\text { Natural circulation inside } \\
\text { containment }\end{array}$ & $\begin{array}{l}\text { CVH/FL may be appropriate. Detailed and judicious } \\
\text { nodalization needed. }\end{array}$ \\
\hline Stratification effects & $\begin{array}{l}\text { Same as above. Processes such as hot gaseous plumes } \\
\text { entering containment volumes require treatments different } \\
\text { than the control volume flow path approach. }\end{array}$ \\
\hline Failure of containment & $\begin{array}{l}\text { Knowledge of leakage size/location exists. The CF/TF } \\
\text { approach is very versatile. }\end{array}$ \\
\hline
\end{tabular}


Table D.IIc

Summary of Review Findings (Melt Relocation, Slump, and Lower Plenum Interval)

\begin{tabular}{|c|c|}
\hline Dominant Phenomena & Review Findings \\
\hline \multicolumn{2}{|l|}{ In-Vessel Phase } \\
\hline $\begin{array}{l}\text { Debris quenching, steam spike, hydrogen } \\
\text { generation and discharge to containment }\end{array}$ & $\begin{array}{l}\text { More appropriate to COR package. However, } \\
\text { provided models can be introduced for degraded } \\
\text { core flooding then RCS thermal-hydraulics, steam } \\
\text { transport, and discharge behavior are predictable } \\
\text { within CVH/FL framework. }\end{array}$ \\
\hline $\begin{array}{l}\text { In-vessel stratification (effect of non- } \\
\text { condensables) }\end{array}$ & Same as previous interval (Table D-IIb). \\
\hline $\begin{array}{l}\text { Impact of in-vessel blockage and flow } \\
\text { diversion }\end{array}$ & $\begin{array}{l}\text { CVH/FL package cannot simulate the impact of } \\
\text { blockage within the core region. Use of CFs/TFs } \\
\text { may be possible for limited cases, but not as a } \\
\text { general modeling feature. The current CVH/FL } \\
\text { package can only allow for redistribution of flow } \\
\text { between parallel channels through plena. Cross } \\
\text { flow diversion between open flow channels } \\
\text { (PWRs) cannot be calculated. }\end{array}$ \\
\hline $\begin{array}{l}\text { Natural circulation (in-vessel, upper } \\
\text { plenum, loops, steam generators) }\end{array}$ & Same as previous interval (Table D-IIb). \\
\hline $\begin{array}{l}\text { Loop seals, steam generator circulation and } \\
\text { plenum mixing }\end{array}$ & $\begin{array}{l}\text { The model may be able to predict loop seal clearing } \\
\text { for PWRs. However, unaware of any calculations. } \\
\text { Other concerns for modeling of natural circulation } \\
\text { apply. }\end{array}$ \\
\hline \multicolumn{2}{|l|}{ Ex-Vessel Phase } \\
\hline Same as previous interval (Table D-IIb) & \\
\hline
\end{tabular}


Table D-IId

Summary of Review Findings (Post-Vessel Failure Interval)

\begin{tabular}{|l|l|}
\hline Dominant Phenomena & Review Findings \\
\hline \multicolumn{2}{|l|}{ In-Vessel Phase } \\
\hline $\begin{array}{l}\text { Depressurization, discharge/blowdown } \\
\text { Natural circulation between in-vessel and } \\
\text { ex-vessel }\end{array}$ & $\begin{array}{l}\text { CVH/FL appears reasonable. } \\
\text { Within limitations of CVH/FL identified earlier, the } \\
\text { CVH/FL framework should allow prediction of } \\
\text { these processes. Again, the HS and RN physics are } \\
\text { strongly coupled to this process. }\end{array}$ \\
\hline Ex-Vessel Phase & $\begin{array}{l}\text { Agatn provided boundary conditions are given } \\
\text { through FDI, CAV, COR, FCI, high-pressure melt } \\
\text { ejection, and direct energy transfer rates; CVH/FL } \\
\text { should be able to predict steam spike and other } \\
\text { pressurization processes. Again an improved } \\
\text { numerical coupling is crucial. }\end{array}$ \\
\hline $\begin{array}{l}\text { Steam spike and containment } \\
\text { pressurization (also see FDI) }\end{array}$ &
\end{tabular}

Table D-IIIa

Summary of Hydrodynamics Model and Package Adequacy

\begin{tabular}{|l|l|l|l|l|l|}
\hline $\begin{array}{l}\text { Dominant } \\
\text { Phenomena }\end{array}$ & $\begin{array}{l}\text { MELCOR } \\
\text { Physics }\end{array}$ & $\begin{array}{l}\text { MELCOR } \\
\text { Numerics }\end{array}$ & $\begin{array}{l}\text { Knowledge } \\
\text { Base }\end{array}$ & $\begin{array}{l}\text { Importance } \\
\text { Category }\end{array}$ \\
\hline \multicolumn{5}{|l|}{} \\
\hline \multicolumn{5}{|l|}{ Initial Transient Depletion and Heatup Interval } \\
\hline $\begin{array}{l}\text { Discharge and } \\
\text { blowdown }\end{array}$ & Correct & Correct & Good & High & 1 \\
\hline $\begin{array}{l}\text { Evaporation at } \\
\text { pool/atmosphere }\end{array}$ & Correct & Correct & Good & High & 1 \\
\hline $\begin{array}{l}\text { Condensation at } \\
\text { pool/atmosphere }\end{array}$ & Not correct & Correct & Good & High & 4 \\
\hline $\begin{array}{l}\text { Evaporation and } \\
\text { condensation on } \\
\text { structures (HS) }\end{array}$ & $\begin{array}{l}\text { Not correct } \\
\text { (HS) }\end{array}$ & $\begin{array}{l}\text { Potential } \\
\text { problem area } \\
\text { (PPA) }\end{array}$ & Good & High & 4 \\
\hline $\begin{array}{l}\text { BWR core and } \\
\text { PW'R pressurizer } \\
\text { sprays }\end{array}$ & $\begin{array}{l}\text { Not modeled } \\
\text { (CF/TF) }\end{array}$ & NA & Good & Low & 7 \\
\hline $\begin{array}{l}\text { Pressurizer } \\
\text { drainage (PWRs) }\end{array}$ & $\begin{array}{l}\text { Not } \\
\text { correct }\end{array}$ & Correct & Good & High & 4 \\
\hline
\end{tabular}


Table D-IIIb

Summary of Hydrodynamics Model and Package Adequacy (Cont.)

\begin{tabular}{|c|c|c|c|c|c|}
\hline $\begin{array}{l}\text { Dominant } \\
\text { Phenomena }\end{array}$ & $\begin{array}{l}\text { MELCOR } \\
\text { Physics } \\
\end{array}$ & $\begin{array}{l}\text { MELCOR } \\
\text { Numerics } \\
\end{array}$ & Knowledge & Importance & $\begin{array}{l}\text { Classification } \\
\text { Category }\end{array}$ \\
\hline \multicolumn{6}{|c|}{ Core Uncovery Interval (In-Vessel Phase) } \\
\hline $\begin{array}{l}\text { Transport and effects } \\
\text { of noncondensables }\end{array}$ & Partial & PPA & Uncertain & High & 2 \\
\hline $\begin{array}{l}\text { RCS natural } \\
\text { circulation }\end{array}$ & Partial & PPA & Good & High & 1 \\
\hline $\begin{array}{l}\text { In-vessel, hot leg, } \\
\text { steam generator } \\
\text { circulation }\end{array}$ & None & $\overline{N A}$ & $\overline{\text { Good }}$ & $\mathrm{High}$ & 4 \\
\hline $\begin{array}{l}\text { Evaporation at } \\
\text { pool/atmosphere }\end{array}$ & Correct & Correct & Good & High & 1 \\
\hline $\begin{array}{l}\text { Condensation at } \\
\text { pool/atmosphere }\end{array}$ & Not correct & Correct & Good & High & 4 \\
\hline $\begin{array}{l}\text { Evaporation and } \\
\text { condensation on } \\
\text { structures (HS) }\end{array}$ & $\begin{array}{l}\text { Not correct } \\
(\mathrm{HS})\end{array}$ & PPA & Good & High & 4 \\
\hline \multicolumn{6}{|c|}{ Core Uncovery Interval (Ex-Vessel Phase) } \\
\hline $\begin{array}{l}\text { Transport and effects } \\
\text { of noncondensables }\end{array}$ & Partial & PPA & Uncertain & High & 2 \\
\hline $\begin{array}{l}\begin{array}{l}\text { Containment natural } \\
\text { circulation }\end{array} \\
\end{array}$ & Partial & $\mathrm{PPA}$ & Good & High & 1 \\
\hline \begin{tabular}{|l|} 
Stratification \\
\end{tabular} & \begin{tabular}{|l|} 
Partial \\
\end{tabular} & PPA & Uncertain & Low & 7 \\
\hline $\begin{array}{l}\text { Containment failure } \\
\text { and leaks }\end{array}$ & $\begin{array}{l}\text { Correct } \\
\text { (CF/TF) }\end{array}$ & PPA & Good & High & 1 \\
\hline
\end{tabular}


Table D.IIIc

Summary of Hydrodynamics Model and Package Adequacy (Cont.)

\begin{tabular}{|c|c|c|c|c|c|}
\hline $\begin{array}{l}\text { Dominant } \\
\text { Phenomena }\end{array}$ & $\begin{array}{l}\text { MELCOR } \\
\text { Physics } \\
\end{array}$ & $\begin{array}{l}\text { MELCOR } \\
\text { Numerics } \\
\end{array}$ & $\begin{array}{l}\text { Knowledge } \\
\text { Base }\end{array}$ & Importance & $\begin{array}{l}\text { Classiffication } \\
\text { Category } \\
\end{array}$ \\
\hline \multicolumn{6}{|c|}{ Melt Relocation, Slump, and Lower Plenum Interval (In-Vessel Phase) } \\
\hline $\begin{array}{l}\text { Quenching, spike, } \\
\text { and discharge }\end{array}$ & Correct & Correct & Good & High & 1 \\
\hline Stratfication & Partial & PPA & Uncertain & High & 7 \\
\hline $\begin{array}{l}\text { Blockage and flow } \\
\text { diversion }\end{array}$ & $\begin{array}{l}\text { Partial } \\
\text { (CF/TF) } \\
\end{array}$ & NA & Uncertain & High & 5 \\
\hline $\begin{array}{l}\text { RCS natural } \\
\text { circulation }\end{array}$ & Partial & PPA & Good & $\mathrm{High}$ & 1 \\
\hline $\begin{array}{l}\text { In-vessel, hot leg, } \\
\text { steam generator } \\
\text { circulation }\end{array}$ & None & $\mathrm{NA}$ & Good & High & 4 \\
\hline \multicolumn{6}{|c|}{ Post-Vessel Failure Interval (In-Vessel Phase) } \\
\hline $\begin{array}{l}\text { Depressurization } \\
\text { discharge and } \\
\text { blowdown }\end{array}$ & Correct & Correct & Good & High & 1 \\
\hline $\begin{array}{l}\text { Natural circulation } \\
\text { between vessel and } \\
\text { containment }\end{array}$ & Partial & PPA & Good & High & 1 \\
\hline \multicolumn{6}{|c|}{ Post-Vessel Failure Interval (Ex-Vessel Phase) } \\
\hline $\begin{array}{l}\text { Steam spike and } \\
\text { pressurization }\end{array}$ & Correct & Correct & Good & High & 1 \\
\hline
\end{tabular}

D-43


Table D.IV

Prioritization of Review Findings for CVH/FL Packages

\begin{tabular}{|l|l|}
\hline$\cdot$ & Numerical problems and code reliability \\
\hline & $\begin{array}{c}\text { Natural circulation: } \\
\text { - In-vessel (core and upper plenum) } \\
\text { - Countercurrent flows (PWR hot leg) } \\
\text { - Steam generator tubes and plena }\end{array}$ \\
\hline & Impact of blockage formation on flow diversion \\
\hline$\cdot$ & Condensation at pool/atmosphere interface \\
\hline$\cdot$ & Pressurizer drainage model \\
\hline Reflood \\
\hline Specific ESF models \\
- BWR core sprays \\
- PWR ice condensers \\
\hline Compendium of sensitivity studies \\
- Parameter/model option sensitivities \\
- Separate effects and integral data \\
- Comparison to other types of codes \\
\hline Improved documentation \\
- Specific guidance to users \\
- Basis for correlations and models
\end{tabular}

Calculations performed to date for both BWRs and PWRs have shown that the CVH/FL modeling approach appears to be adequate under most severe accident conditions; however, considerable insights on the part of code users are required. The reliability of MELCOR calculated results is highly questionable in light of the existing numerical problems, because it is often difficult to distinguish between numerical vs physical behavior (the origin of these numerical problems is not clearly known, even though some potential problem may exist in the CVH/FL package numerics, especially in terms of CVH/FL coupling with the other MELCOR phenomenological modules). 


\section{REFERENCES}

D-1, R, K, Cole, Jr., "Thermal Hydraulics Packages (CVH and FL) Reference Manual," Sandia National Laboratories unnumbered report (February 17, 1989),

D-2. R. M. Summers et al., "MELCOR 1.8.0: A Computer Code for Nuclear Reactor Severe Accident Source Term and Risk Assessment Analyses," Sandla National Laboratories report SAND9)-0364, R3 (NUREG/CR-5531) (Octobet 199()).

D-3. A. Camp et al., "HECTR Version 1.0 User's Manual," Sandia National Laboratories report SAND84-1522 (NUREG/CR-3913) (February 1985).

D-4. K. K. Murata et al., "User's Manual for CONTAIN 1.1: A Computer Code for Severe Nuclear Reactor Accident Containment Analysis," Sandia National Laboratories report NUREG/CR-5026 (November 1989).

D-5. P. C. Owczarski et al., "Technical Bases and User's Manual for the Prototype of a Suppression Pool Aerosol Removal Code (SPARC)," Pacific Northwest Laboratories report NUREG/CR-3317 (May 1985).

D-6. G. M. Martinez, "MELCOR Post-Test Calculations of the HDR Experiment," Sandia National Laboratories informal report (September 29, 1989).

D.7. M. I. Robertson,"Validation of Control Volume Thermal Hydraulic Modelling," CSNI/CEC Workshop on Aerosol Behavior in the Containment, Fontenay-aux-Roses, France, November 1990.

D-8. I. K. Madni, "MELCOR Lessons Learned: A User Perspective," Brookhaven National Laboratory informal report (January 1991).

D-9. I. K. Madni, "MELCOR Simulation of Long-Term Station Blackout at Peach Bottom," Proceedings of the Water Reactor Safety Meeting, Rockville, October 1990.

D-10. S. L. Chan, H. P. Isaak, U. Schmocker, and M. Khatib-Rahbar, "Characterization of Severe Accidents Using MEI,COR: A Perspective," Paper Presented at the Cooperative Severe Accident Research Program (CSARP) Semi-Annual Meeting, Bethesda, Maryland, May 6-10, 1991.

D-11. E. A. Boucheron and J. E. Kelly, "MELCOR Analysis of the Three Mile Island Unit 2 Accident," Nuclear Technology 87, p. 1050 (December 1989).

D-12. E. A. Boucheron and J. E. Kelly, "MELCOR Analysis of the TMI-2 Accident," Paper Presented at the ANS/ENS TMI-2 International Topical Meeting, Washington, DC, October 1988.

D-13. S. Dingman, "MELCOR Blind Calculations for HDR Tests 31. 4 and 31.5," Sandia National Laboratories informal undated report.

D-14. L. A. Miller et al., "N Reactor Probabilistic Risk Assessment Supporting Calculations," Sandia National Laboratories report SAND89-2101/1 of 3 (April 199()). 
D-15 G. M. Martinez, "MELCOR Calculations of ISP28 SFD PHEBUS Test: B9+," Sandia National Laboratories informal report (December 14, 1990).

D-16 I. K. Madni, "MELCOR Modeling of the PBF Severe Fuel Damage Test 1-4," Brookhaven National Laboratory report BNL-NUREG-44503 (1990).

D-17 G. L. Sozzi, "Experimental Data Set No. 21 Level Swell and Void Fraction Measurement during Vessel Blowdown Experiments," GE Nuclear Energy, USA undated report.

D-18 R. M. Summers, "MELCOR Improvement Needs Assessment," Sandia National Laboratories letter report to R. Foulds, US Nuclear Regulatory Commission (October 17, 1990). 


\section{ANNEX D-1}

\section{MELCOR Input for Simulation of GE Vessel Blowdown Tests}

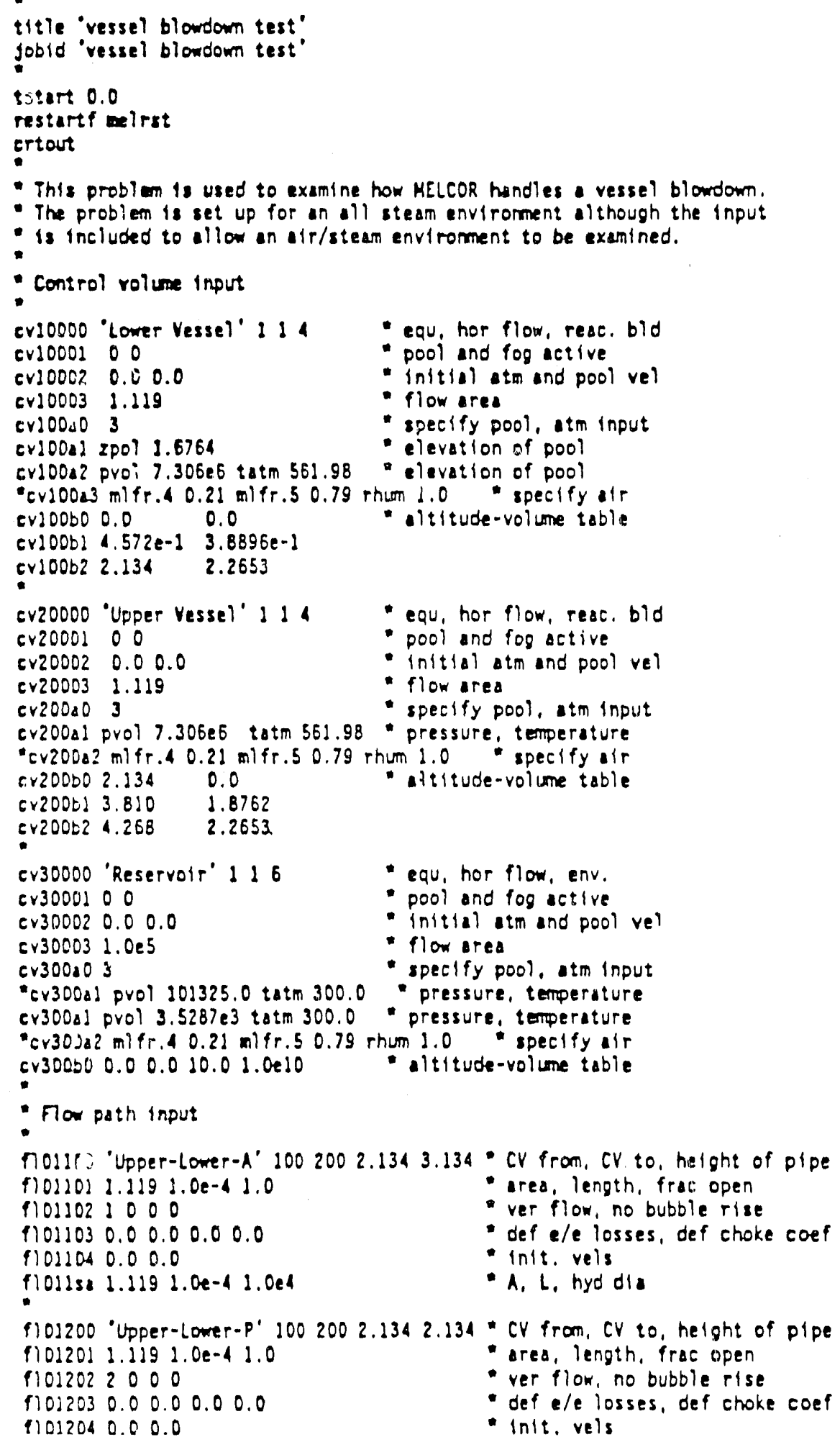

- Init. vels 


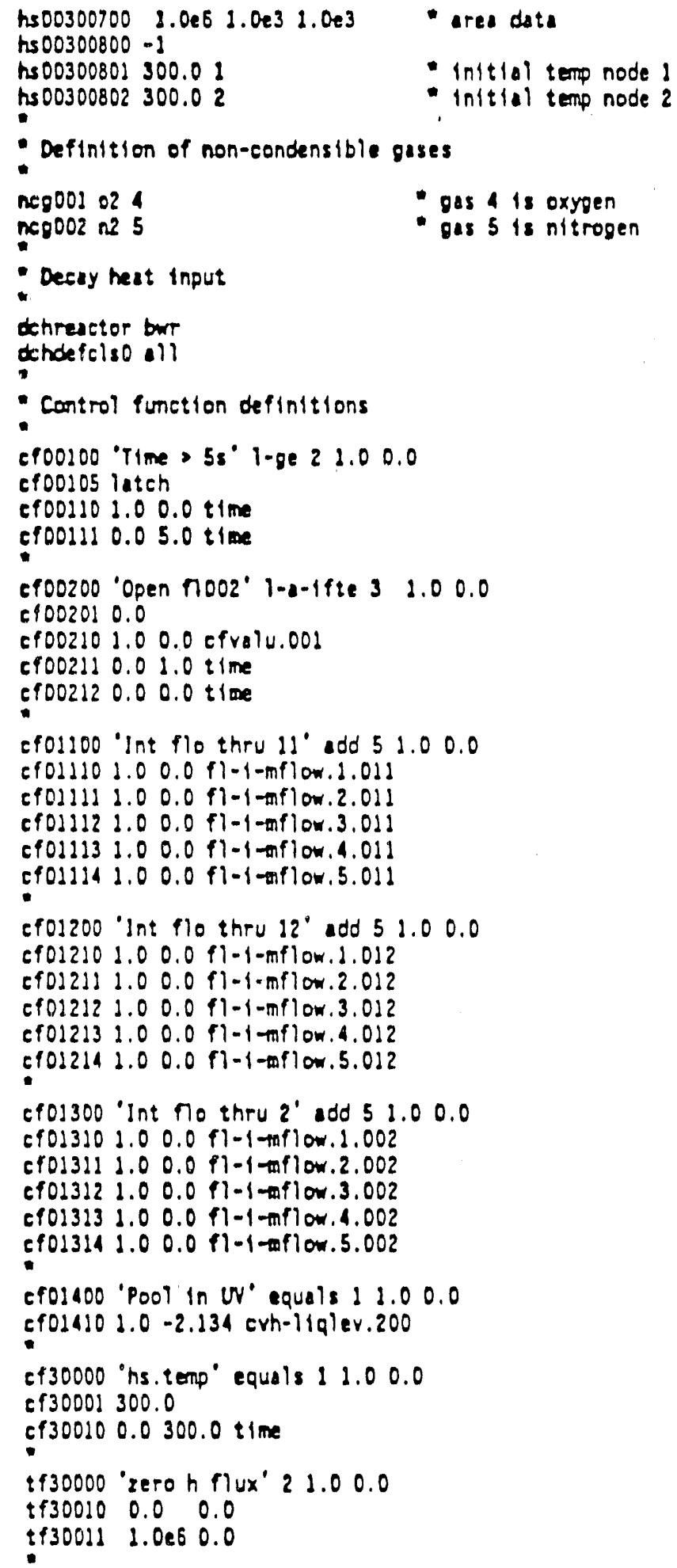




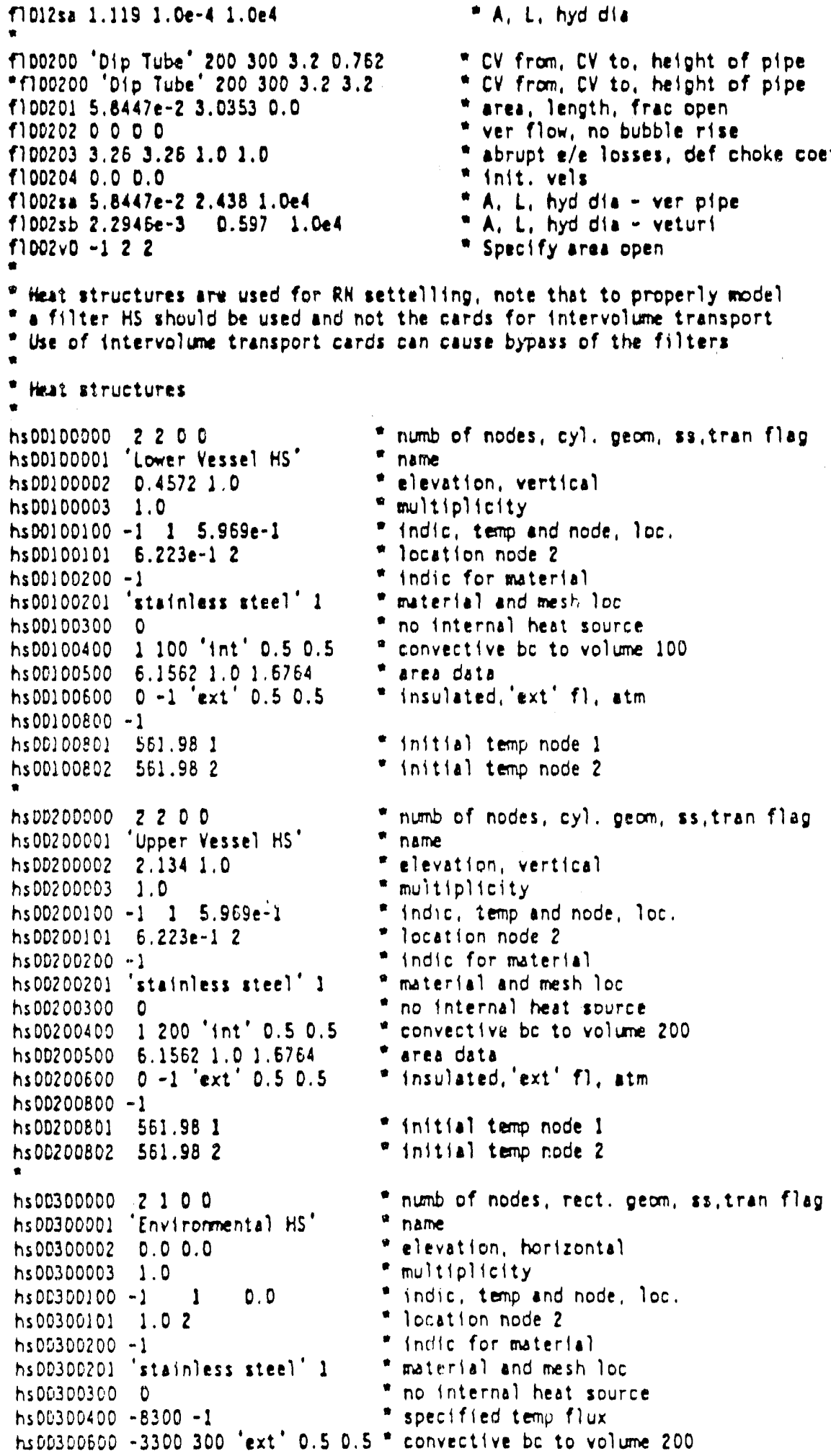

hs00300600-3300 300 'ext' 0.50 .5 " convective be to volume 200

- numb of nodes, cyl. geom, ss, tran flag

- name

- elevation, vertical

- multiplicity

- Indic, temp and node, loc.

- location node 2

- Indic for naterial

- materlel and mesti loc

- no internal heal source

- convective be to volume 100

- area data

- insulated, 'ext' fl, atm

- Initial temp node !

- Initial temp node 2

- numb of nodes, cyl. geom, ss,tron flag

- name

- elevation, vertical

- muitiplicity

- Indic temp and node, loc.

- location node 2

- Indic for materlal

- material and mesh loc

- no internal heat source

- convectiva be to volume 200

- area data

- insulated, 'ext' fl, atm

- inftial temp node

- initial temp rode?

- numb of nodes, rect. geom, ss, tran flag

- name

- Eevation, horizontal

- multiplicity

- indic, temp and node, loc.

- location node 2

- Indic for materlal

- materlal and mesh loc

- no internal heat source

- spectified temp flux 


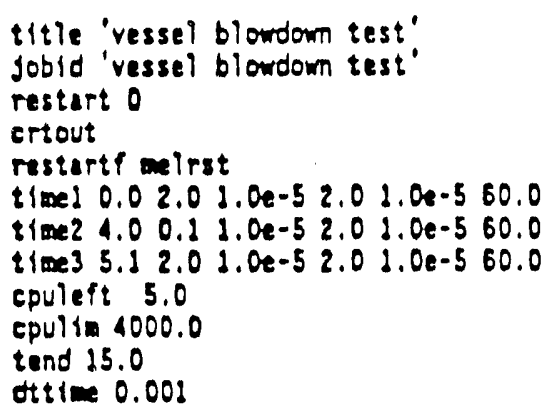

ottim 0.001 
ANNEX D.2

\section{MELCOR Input for Simulation of a Simple Condensation Problem}

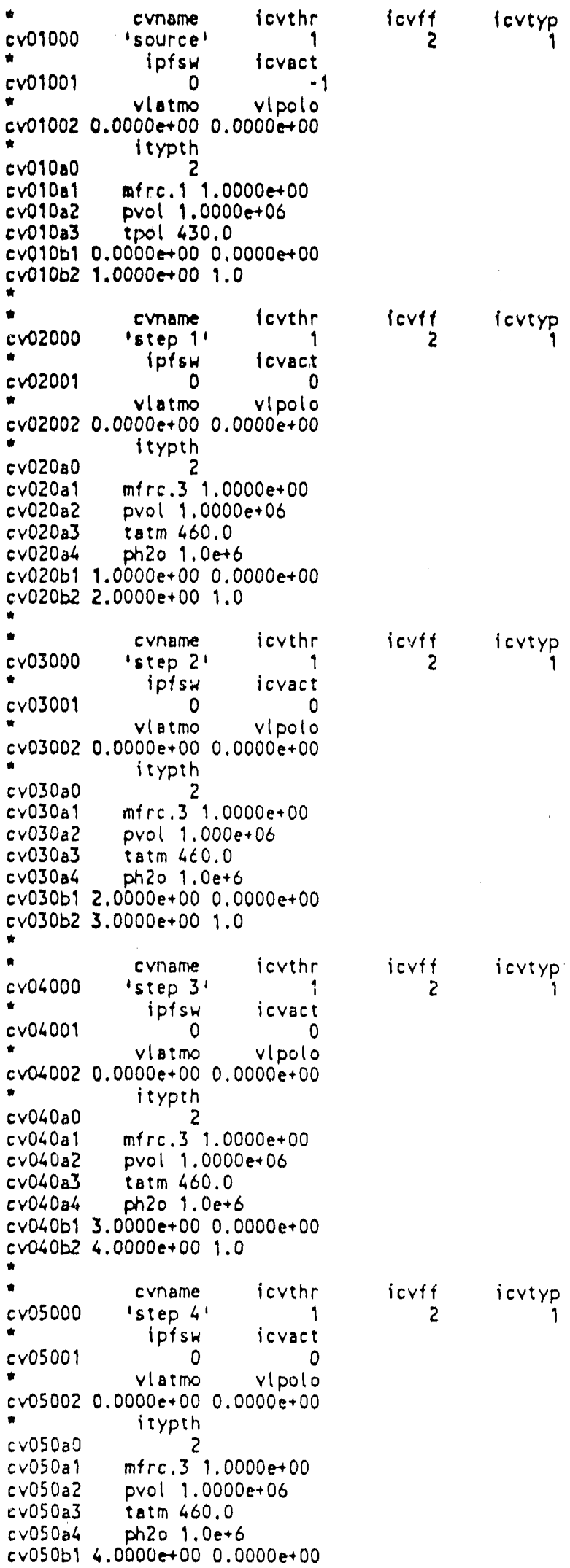




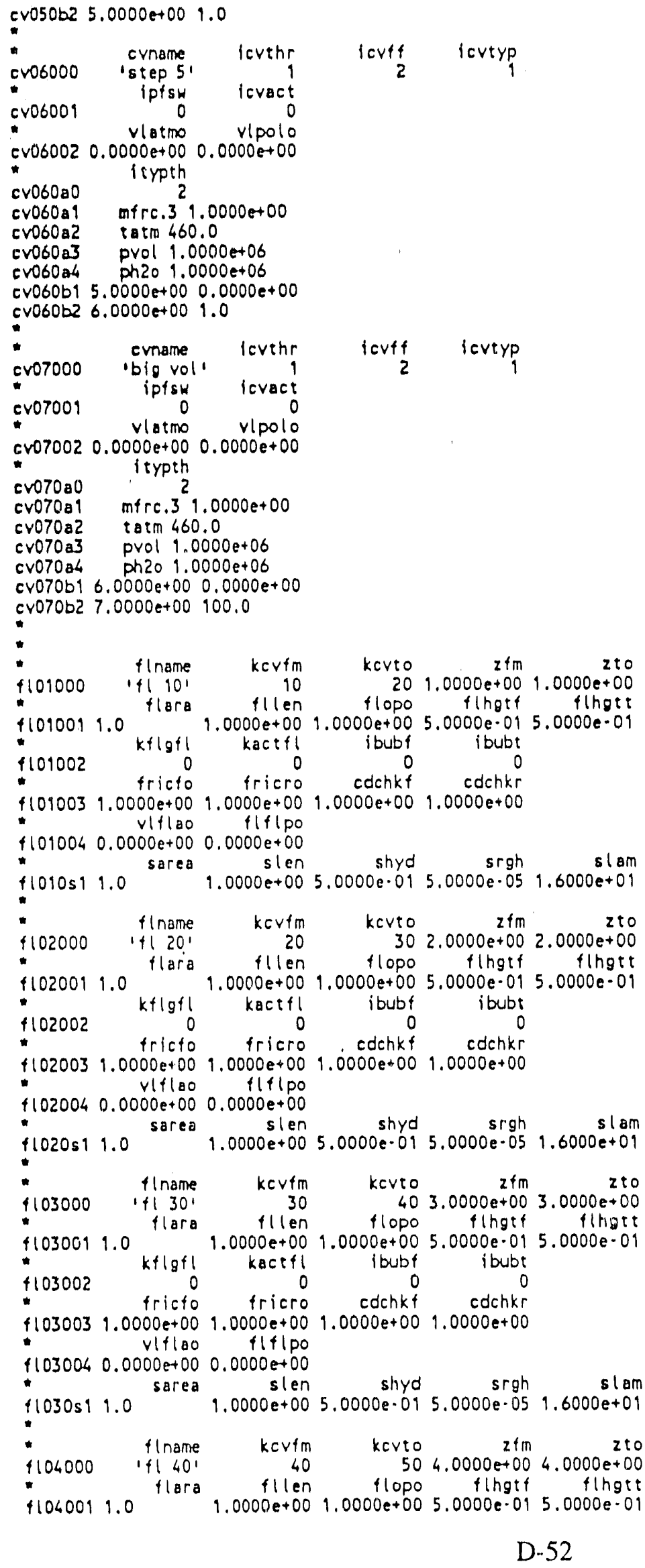

D. 52 


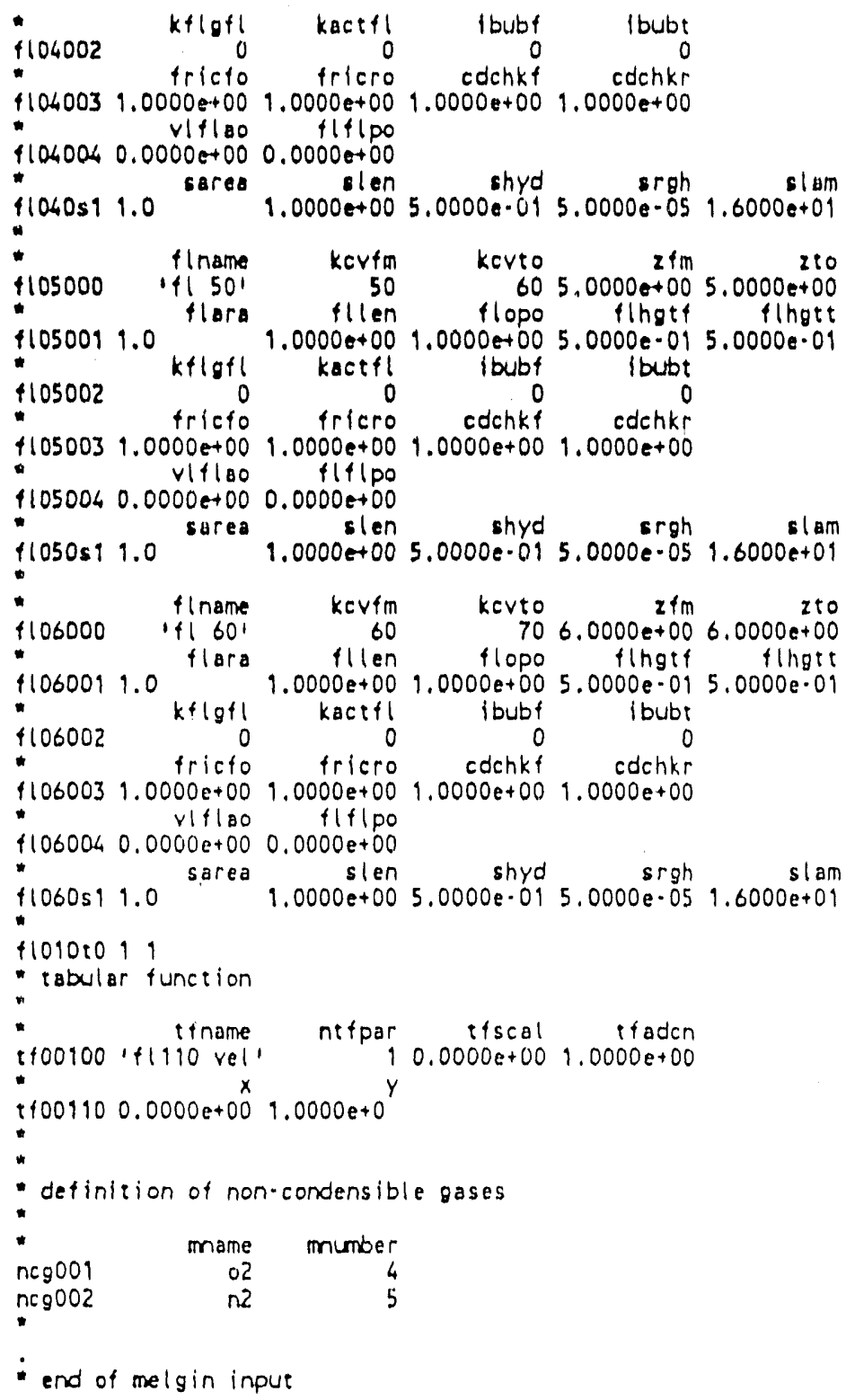




\section{APPENDIX E \\ Heat Structure (HS) Package}

by

\section{K. Dhir}

Modeling the thermal response of plant structures is important in the overall simulation of severe accidents. Phenomenological package HS models the thermal response of heat structures and models mass and heat transfer between heat structures and control volume pools and atmospheres. This package treats conduction, condensation, convection, and radiation, as well as degassing of unlined concrete. MELCOR heat structures also serve as settling surfaces for radionuclide aerosols and deposition surfaces for condensate films. A summary of the phenomenological models in the HS package is given in Table E-I. The following conclusions are primarily based on the information provided in Refs. E-1, E-2, and E-3.

\section{E.1. MODEL DESCRIPTION AND PEDIGREE}

A one-dimensional transient conduction equation with volumetric heating is solved to obtain temperature distribution in intact structures. Provisions for specifying temperature, heat flux, and convective boundary conditions exist. Convective heat-transfer coefficients are obtained by using steady-state correlations reported in the literature.

\section{E.2. IMPLEMENTATION WITHIN MELCOR}

The HS package serves as a building block for several other packages in the code. The geometry, physical dimensions, and thermophysical properties of heat structures are user input.

\section{E.3. RESULTS OF MODEL AND PARAMETER SENSITIVITY STUDIES} time-step size.

Transient conduction results have been found to show some sensitivity to node size and

\section{E.4. RESULTS OF MODEL BENCHMARKING/VALIDATION ACTIVITIES}

Three relatively simple steady-state and transient one-dimensional configurations for which analytical solutions are available in the literature have been used to validate the numerical results from the HS package. The test cases are (1) transient conduction in a slab or cylinder when the lumped-capacity method is applicable, (2) steady-state conduction in an annular geometry, and (3) transient conduction in a semi-infinite slab with a convective boundary condition. For all cases, a good agreement of the numerical results with the analytical solutions was noted. However, oscillations in temperatures in the semi-infinite slab were observed. The magnitude of temperature oscillations was dependent on the node and time-step size.

\section{E.5. IDENTIFIED DEFICIENCIES AND PLANNED IMPROVEMENTS}

Some convective and boiling correlations are outdated. The condensation process is poorly modeled. No rationale is given either for choosing the characteristic length in a particular correlation or for using steady-state correlations in a transient process. Limitations of the 
correlations with respect to their applicability are not discussed. Flow boiling is not considered. Melting and relocation of heat structures outside of the COR package are not allowed.

\section{E.6. SUMMARY OF FINDINGS}

Methodology is reasonable but in some cases the correlations imported from the literature have not been used properly. Limitations and assumptions made in the use of correlations have not been clearly stated. Modeling of condensation in the presence of noncondensables is physically not correct. No models or correlations are included for flow boiling. Melting or relocation of heat structures is not allowed.

\section{E.7. IMPORTANCE ASSESSMENT}

The model-related uncertainties can influence in a significant way the rate at which heat is stored in RCS, melting and relocation of structures, failure of the surge line and steam generator tube, and the pressurization of the containment. The inaccuracies in calculations of the pressure in the containment can be critical if the maximum predicted pressures are close to the design pressure. The inability to calculate in a realistic manner the heatup rate of the RCS can lead to uncertainty in the prediction of alternate failure paths. Noninclusion of melting and relocation of structures in the reactor vessel can lead to errors in the mass of the molten material and its composition. The significance of all of these uncertainties will be plant- and accident-sequence specific.

The following pages summarize the findings for the individual models appearing in the HS phenomenological package. 


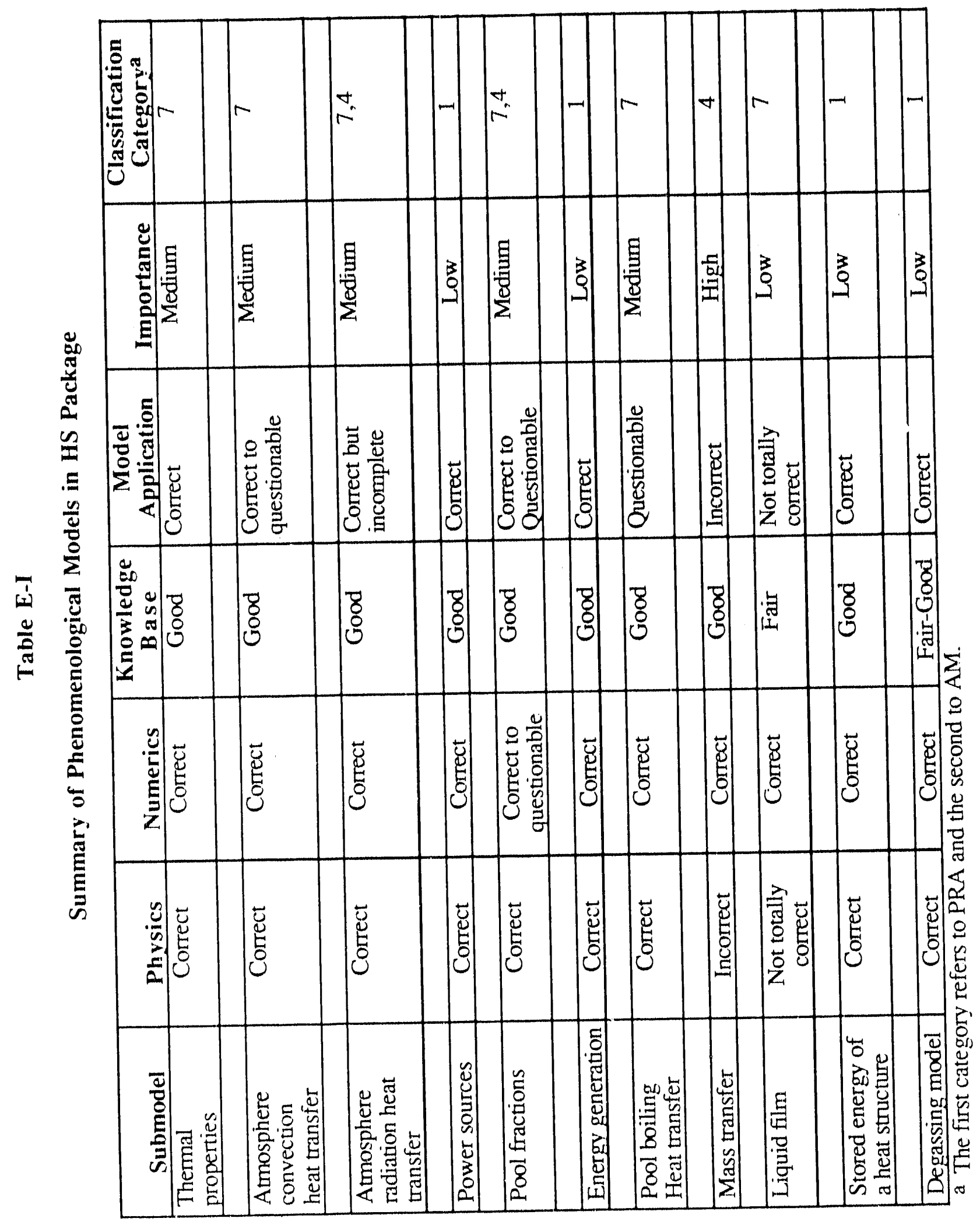

E-3 


\section{MODEL 'TITLE: 'THERMAL PROPER'TIES}

\section{Model Description and Pedigree:}

Only thermal conductivity and volumetric heat capacity are considered. T'emperaturedependent values are obtalned from the material properties package, for a degassing material, pseudo-volumetric heat capacity is defined.

\section{Implementation Within MELCOR:}

Radiative properties must be specified through user input.

\section{Results of Model and Parameter Sensitivity Studies:}

None found.

Results of Model and Benchmarking/Validation Activities:

None found.

\section{Identified Deficiencies and Planned Improvements:}

After degassing, the properties are not corrected for loss of decomposed material. Surface properties such as emissivity and absorptivity need specification by the user. User guidelines are not given in sufficient detuil.

\section{Summary of Findings:}

Adequate treatment as long as temperatures remain low and surface properties do not change as a result of surface oxidation and degradation.

\section{Importance Assessment:}

Heatup or cooldown of structures cannot be calculated accurately.

Technical Adequacy:

Category 7. 


\section{MODEL TITLE: A'TMOSPHERE CONVECTION HEA'T 'TRANSFER}

\section{Model Description and Pedigree:}

Both internal and external convection under free and forced convection conditions are considered, Steady-state cortelations reported in heat-transfer texibooks are used. Rectangular, cylindricul, and spherical geometries can be treated.

\section{Implementation Within MEL.COR:}

A table of empirical constants used in free and forced convection correlations for different geometries is given.

\section{Results of Model and Parameter Sensitivity Studies:}

None found.

\section{Results of Model Benchmarking/Validation Activities:}

The correlations are based on steady-state data obtained under the reported experimental conditions and range of experimental parameters.

\section{Identified Deficiencies and Planned Improvements:}

In some cases, the correlations are outdated. No rationale is given for using steady-state correlations in transient or yuasi-static conditions or for developing flows. Boundary conditions and geometrical configurations to which correlations are applied may not be exactly the same as those used in the experiments. No basis is given as to how the characteristic length is chosen in a given correlation. The internal natural convection aspect ratio can be important but is not considered. Some of the correlations are not applicable to the geometries considered in the code. The code developers plan to remove the latter deficiency. Mixed convection is not treated in " proper manner.

\section{Summary of Findings:}

Application of correlations to nonsimilar and transient situations will lead to inaccurate results.

\section{Importance Assessment:}

Will play an important role if timing of such events as surge line failure and steam generator tube rupture is to be calculated.

Technical Adequacy:

Categories 7 and 4. 


\section{MODEL TITLE: A'TMOSPHERE RADIATION HEA'T TRANSFER}

\section{Model Description and Pedigree:}

Equivalent band or gray gas model optlons are employed in the code. The models have been obtaned from the open literuture.

\section{Implementation Within MELCOR:}

User has the flexibility of using elther option. The radiative properties of the pool and structure are also specified by the user. The model has not been implemented correctly and has arbitrary specification and view factors.

\section{Results of Model and Parameter Sensitivity Studies:}

None found.

Results of Model Benchmarking/Validation Activities:

None found.

\section{Identified Deficiencies and Planned Improvements:}

No guidance is given to the user on the choice of an option. Large uncertainties exist in specification of emissivities and absorptivities. Structure-to-structure radiation exchange is not considered and no information is given on the shape factors.

Summary of Findings: properties.

The model is adequate except that large uncertainties may exist with respect to radiative Importance Assessment: accurately.

Heat loss from licuid pools and structure heatup or cooldown rates may not be predicted

\section{Technical Adequacy:}

Categories 7 and 4. 


\section{MODEL 'TI'TLE: POWER SOURCES}

\section{Model Description and Pedigree:}

The model includes both external and internal heat sources in a heat structure. 'The internal heat sources cun be spatial and time dependent. Surface heat fluxes are user specified or are obtained through interactions with other modules. Surface heal fluxes are calculated before callculation of temperature.

\section{Implementation Within MELCOR:}

The magnitude, spatial, and time variations of the internal heat sources and surface heal fluxes are specified by the user.

\section{Results of Model and Parameter Sensitivity Studies:}

Nothing specilic found.

\section{Results of Model Benchmarking/Validation Activities:}

Nothing specific found.

\section{Identified Deficiencies and Planned Improvements:}

It is not clear as to how the effect of structure temperature on surface heat flux from an external heat source is included. The model does not distinguish surface heat flux resulting from radiation or resulting from deposited fission products.

\section{Summary of Findings:}

Adecjuate except when feedback between structure temperature, structure radiative properties, and surface heat flux imposed from external sources becomes important.

\section{Importance Assessment:}

The contribution to the uncertainty in the final results is expected to be small.

\section{Technical Adequacy:}

Category 1. 


\section{MODEL TITLE: POOL FKACTIONS}

\section{Model Description and Pedigree:}

The fraction of the surface that is exposed to a liquid pool and the environment is obtained through geometrical relationships. The concept of critical pool fractions is used to distinguish between the environment and the pool. For use in the code, equal critical fractions for the pool and the environment are recommended.

\section{Implementation Within MELCOR:}

If the pool level drops below the critical fraction (1/2), all of the structure is exposed to the environment. On the other hand, if the pool level is above the critical fraction, all of the structure surface is assumed to be exposed to liquid.

\section{Results of Model and Parameter Sensitivity Studies:}

None found.

\section{Results of Model Benchmarking/Validation Activities:}

Nothing specific found.

\section{Identified Deficiencies and Planned Improvements:}

Concept of critical fractions is unreasonable in transient processes such as boil off and reflooding. In one of the code calculations, the observed difficulties in completely drying out the steam generator could be traced to these deficiencies. The code developers believe that the identified deficiency can be circumvented by increasing the number of heat structures.

\section{Summary of Findings:} unrealistic results.

During reflood and boil off, the use of equal fractions for pool and environment will yield

\section{Importance Assessment:} important.

Not a significant issue unless knowledge of timing of certain events is deemed to be

\section{Techrical Adequacy:}

Categories 7 and 4. 


\section{MODEL TITLE: POOL BOILING HEAT TRANSFER}

\section{Model Description and Pedigree:}

Only saturated pool boiling is considered. Well-tested correlitions reported in the literature for steady-state nucleate boiling, maximum heat flux, minimum heat flux, and film boiling are used. Transition boiling heat fluxes are interpolated between critical and minimum heat fluxes.

\section{Implementation Within MELCOR:} MOD2.

Modeling of film boiling with radiation is not consistent with the modeling in CORCON-

\section{Results of Model and Parameter Sensitivity Studies:}

None found.

\section{Results of Model Benchmarking/Validation Activities:}

None found.

\section{Identified Deficiencies and Planned Improvements:}

Correlations for different geometries are intermixed. Choice of characteristic length in film boiling is left to the user's discretion and no guidance is given to the user. The empirical constant used in the expression for the maximum heat flux is about 20-25\% higher than the accepted value. The properties are not evaluated properly. Radiative contribution in transition boiling does not yield proper limits. The radiation contribution has not been properly included in film boiling. Chemical reactions during film boiling are not considered. Flow boiling is not considered.

\section{Summary of Findings:}

Correlations well documented in the literature have been used. However, they have not been applied in a judicious manner. In film boiling, the radiation contribution has not been included properly. The characteristic length to be used in film boiling has not been specified. No correlations or models are included in flow boiling.

\section{Importance Assessment:}

Deficiencies may lead to inaccuracy in the timing of the events.

\section{Technical Adequacy:}

Category 7. 


\section{MODEL TITLE: MASS TRANSFER}

\section{Model Description and Pedigree:}

Condensation of pure vapor, condensation and evaporation in the presence of noncondensables, and flashing of superheated liquid layers present on heat structures are considered. For condensation of pure vapor, Nusselt's formulation is used. A low mass-transfer limit is invoked for condensation in the presence of noncondensables. Sensible heat transfer from vapor-gas mixture to condensate layer is included. In the Nusselt formulation, the condensation heat-transfer coefficient is arbitrarily increased by about $20 \%$.

\section{Implementation Within MELCOR:} subroutines.

Steam and noncondensable composition in the environment is obtained from other

\section{Results of Model and Parameter Sensitivity Studies:}

None except those obtained indirectly through HDA test predictions.

\section{Results of Model Benchmarking/Validation Activities:}

None except those obtained indirectly through HDA test predictions.

\section{Identified Deficiencies and Planned Improvements:}

No justification is given for the use of laminar condensation heat-transfer coefficients when the film becomes turbulent. The condensation heat-transfer coefficient obtained from the Nusselt-type analysis has been arbitrarily increased by about $20 \%$. For a structure surface angle of inclination less than $9.7^{\circ}$, an expression for condensation heat-transfer coefficient on a horizontal cylinder is used without any justification. No guidance is given to the user as to how to choose the characteristic length used in the expression for condensation heat-transfer coefficients. The model does not account for the condensate film resistance in the presence of noncondensables. Use of expressions based on a low mass-transfer limit will give the wrong results under high masstransfer conditions. The flashing model is deficient in that the evaporation rate is dependent on the time step.

\section{Summary of Findings:}

The model used for condensation in the presence of noncondensables is seriously flawed. Expressions used for heat-transfer coefficients during condensation of pure vapor lack proper justification.

\section{Importance Assessment:}

Predicted steam condensation rates when the thermal resistance of the structure on which condensation occurs does not dominate will he in serious error. This points to the fact that good agreement observed between predictions and IIDA data may be fortuitous.

\section{Technical Adequacy:}

Category 4. 


\section{MODEL TITLE: LIQUID FILM}

\section{Model Description and Pedigree:}

The model calculates steady-state and transient thickness of liquid film on a structure. Thicknesses are obtained from Nusselt-type film condensation coefficients. An arbitrarily specified default value of $50 \times 10^{-6} \mathrm{~m}$ is used for the initial film thickness and for the maximum film thickness.

\section{Implementation Within MELCOR:}

Liquid mass in the film is obtained from other packages in the code. A default value of $50 \times 10^{-6} \mathrm{~m}$ is used for the initial and the maximum thickness of the film.

\section{Results of Model and Parameter Sensitivity Studies:}

None found.

Results of Model Benchmarking/Validation Activities:

None found.

\section{Identified Deficiencies and Planned Improvements:}

A film of constant thickness without any provisions for waves on the film surface is assumed. For surface angles of inclination less than $9.7^{\circ}$, the film thickness is assumed not to depend on the angle of inclination. No rational basis has been given for evaluation of thermophysical properties.

\section{Summary of Findings:}

film.

No mechanistic basis is provided for the specified initial and maximum thickness of the

\section{Importance Assessment:}

The modeling deficiencies may contribute to the uncertainties in condensation or evaporation of fission products.

\section{Technical Adequacy:}

Category 7. 


\section{MODEL TITLE: STORED ENERGY OF A HEAT STRUCTURE}

\section{Model Description and Pedigree:}

The model calculates energy stored in the structure and the liquid film on the surface. The energy content is obtained by integrating over the volume, the product of temperature, density, and specific heat.

Implementation Within MELCOR:

Properties are obtained from input data tables provided by the user.

Results of Model and Parameter Sensitivity Studies:

None found.

Results of Model Benchmarking/Validation Activities:

None found.

Identified Deficiencies and Planned Improvements:

None found.

Summary of Findings:

Model is adequate.

Importance Assessment:

Technical Adequacy:

Category 1. 
MODEL. TITLE: DEGASSING

Model Description and Pedigree:

The model calculates the gas release rate during decomposition of a solid. Over the temperature range of decomposition, the gas release rate is assumed to vary linearly.

Implementation Withis MEHCOR:

The decompoing golid fraction is obtained from the input property data.

Results of Model and Parameter Sensitivity Studies:

None found.

Results of Model Benchmarking/Validation Activities:

None found.

Identified Deficiencies and Planned Improvements:

No significant deficiency is found except that the effect of degassing on other chemical reactions is not included.

Summary of Findings:

Model is adequate.

Importance Assessment:

The uncertainties associated with this model should have little effect on the final results.

Technical Adequacy:

Category 1. 


\section{REFERENCES}

E-1. R. M. Summers, R. K. Cole, Jr., E. A. Boucheron, M. K. Carmel, S. E. Dingman, J. E. Kelly, "MELCOR 1.8.(): A Computer Code for Severe Nuclear Reactor Accident Source Term and Risk Assessment Analyses," Sandia National Laboratories report SAND9()-(0364 (NUREG/CR-5531) (January 1991).

E-2. R. M. Summers, "MELCOR Primer, Version 1.8.()," Sandia National Laboratories unnumbered report (February 17, 1989).

E-3. C. D. Leigh, Editor, "MELCOR Validation and Verification; 1986 Papers," Sandia National Laboratories report SAND86-2689 (NUREG/CR-4830) (March 1987). 


\title{
APPENDIX F \\ Core Heatup and Degradation (COR) Package
}

by

\author{
R. Viskanta and T. J, Haste
}

\section{F.I. MODEL DESCRIPTION AND PEDIGREE}

In MELCOR, the core heatup and degradation processes are modeled by the core heatup and degradation (COR) package. The MELCOR COR package calculates the transient thermal response of the core and lower plenum, internal structures, including the portion of the lower head directly below the core. The package also models the relocation of core and lower plenum structural materials during melting, slumping, and debris formation.

All the thermal behavior models are based on the energy conservation principle and equations derived for the control volume. The material relocation models are based on mass and energy conservation equations. However, some of the heat exchange processes have been neglected. One example of such a process is heat exchange between molten film and the surrounding coolant during candling. In addition, material relocation is assumed to be instantaneous as no allowance is made for the tansit time of the material, which is assumed to be short in comparison to the system time. The velocity of the relocating material is not determined and the momentum equations are not solved.

The COR package uses the lumped-parameter approach instead of the more detailed finite-difference formulation of the mass and energy conservation equations. The modeling framework can be summarized as follows.

- Core and lower plenum regions are divided into concentric axial rings and axial segments.

- Five possible components modeled are (1) intact fuel, (2) cladding, (3) other structures (e.g., control rods or guide tubes), (4) canister walls adjacent to control blades (BWRs), (5) canister walls not adjacent to control blades (BWRs), and (6) particulate debris (as a possible component).

- Each component may include one or more of six materials: (1) $\mathrm{UO}_{2},(2)$ Zircalloy, (3) $\mathrm{ZrO}_{2}$, (4) steel, (5) steel oxide, and (6) control rod poison.

- The nodalization scheme used by the COR package is much finer than the $\mathrm{CVH}$ package.

- A lumped-parameter approach is used for each cell, so that each component is represented by a single equilibrium temperature.

Before core relocation is initiated, the time rate of net energy change of a component is calculated from an energy balance on a control volume as a sum of individual energy sources and 
heat-transfer rates. Conservation of energy in a component is maintained by accounting for variable heat capacity, phase changes, and addition or removal of materials from a component. Decay heat power is calculated from fission product inventories, which are specified by the user and tracked by the RN package. In general, modeling of heat transfer before severe core degradation is initiated is reasonable and at a level appropriate for a fully integrated engineeringlevel computer code that models progression of severe accidents in LWR nuclear power plants. A number of specific concerns have been identified and are discussed in Section F.7.

After the core begins to degrade severely, relocates, and the debris attacks the lower head, the variety of in-vessel processes is so large and complex that the phenomena are either unresolver or currently beyond our understanding. During this phase of severely degraded core LWR accidents the phenomenological issues, which are not well enough understood, have not been quantified in MELCOR. The models describing the phenomena are parametric in nature, and they are not more than educated guesses. The core relocation, blockage, holdup, lower head attack, and breach models appear to represent the code developers' views of the phenomena in severe nuclear reactor accidents. Spotty experimental evidence and lack of theoretical analysis have led to speculation about important phenomena. It appears that too much reliance has been placed on numerical code development and not enough attention given to deterministic physical processes and their description using "zero order" models. Core melt progression and lower head attack models are only shells containing few deterministic details. These models in the COR package are primarily shells for performing parametric calculations employing user-specified parameters without any regard to physics.

A summary of the existing and missing phenomenological models in the COR package is given in Table F-I. Findings for individual models included in COR are discussed in Section F.7.

\section{F.2. IMPLEMENTATION WITHIN MELCOR}

The COR package uses an explicit numerical scheme to advance the thermal state of the core in time. The numerics of the package can be summarized by the following steps:

(1) The COR package is coupled explicitly to the radionuclide $(R N)$, decay heat $(\mathrm{DCH})$, control volume hydrodynamics $(\mathrm{CVH})$, and heat structure (HS) packages. The interface boundary conditions, the transfer of heat and mass, and the heat source terms are treated explicitly.

(2) A lumped-parameter approach is used for component thermal behavior models.

(3) Within the package the physical phenomena are also treated explicitly.

(4) To mitigate numerical instabilities, a subcycling capability has been included to allow COR to take multiple time steps arross a single system cycle.

(5) All heat generation and heat- and mass-transfer rates are evaluated at the beginning of a COR package subcycle based on current temperatures, conditions, and estimated local fluid conditions.

The numerical integration techniques currently implemented within the COR package appear reasonable. However, the explicit coupling scheme between $\mathrm{COR}, \mathrm{DCH}, \mathrm{CVH}, \mathrm{HS}$, and RN packages could be responsible for numerical instabilities reported by several users (Refs. F-1 and F-2). The following additional comments can be made:

(1) Numerical instability is prone to take place when a large time step is used or when a sudden event such as a large mass/heat exchange occurs. 


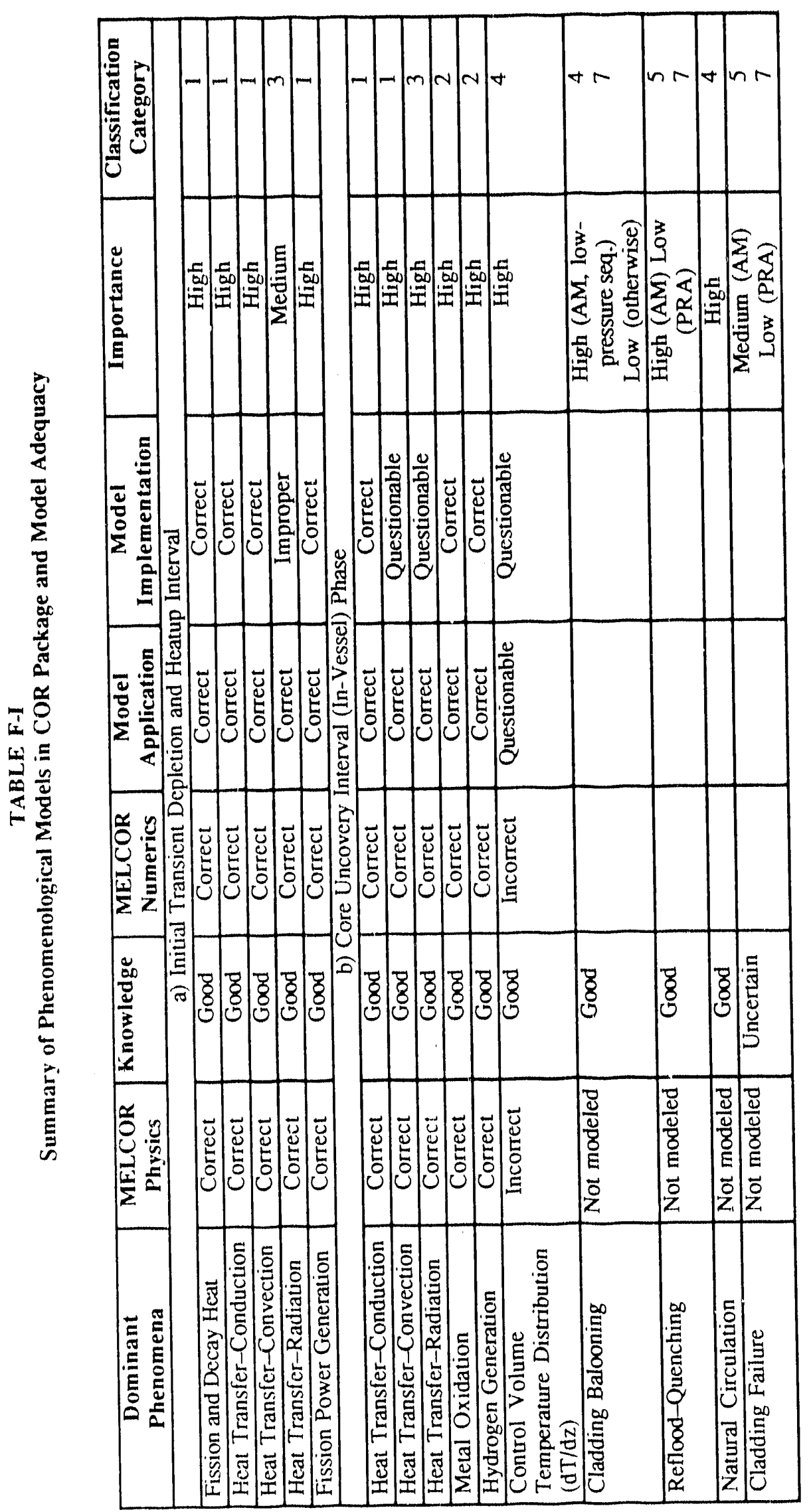




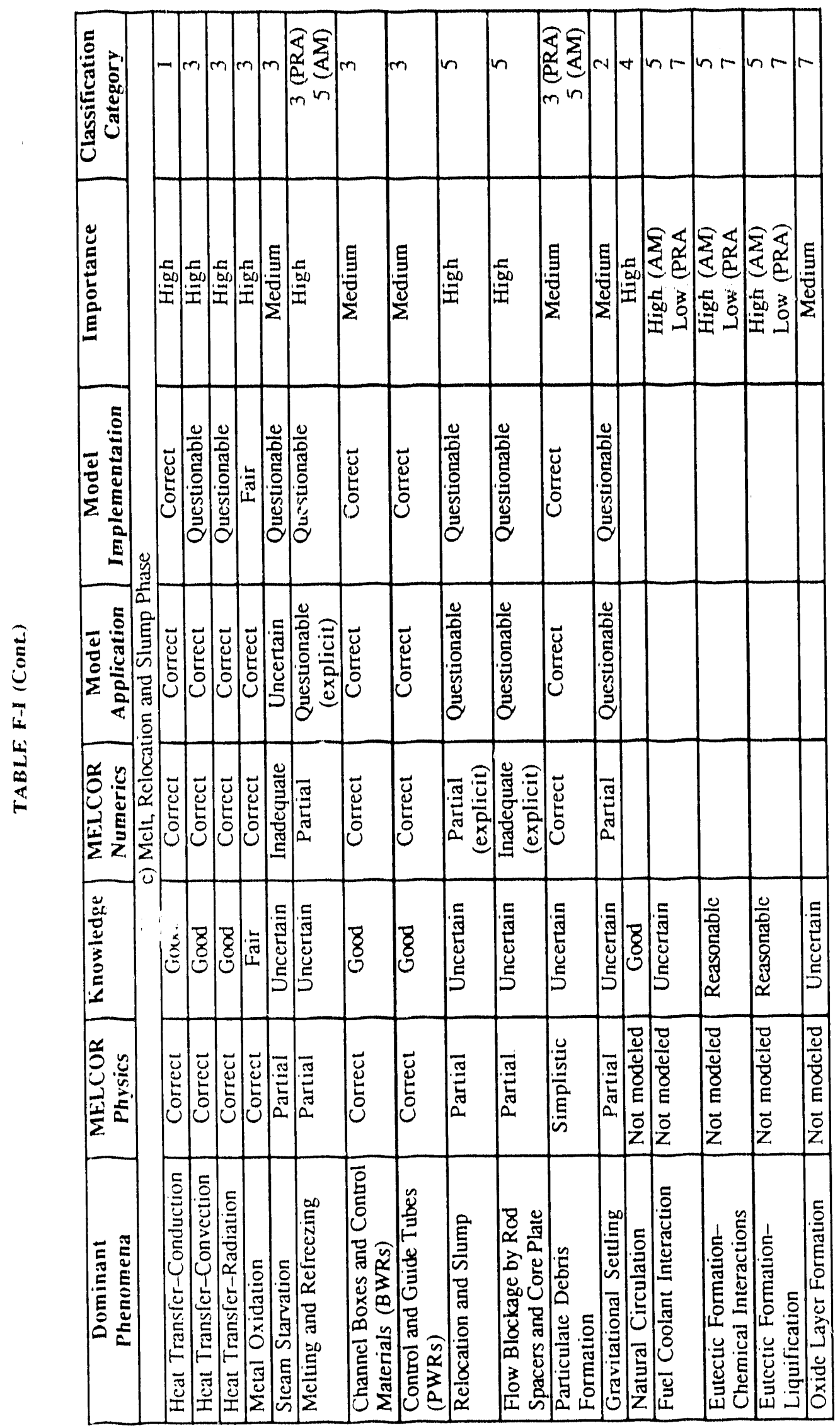




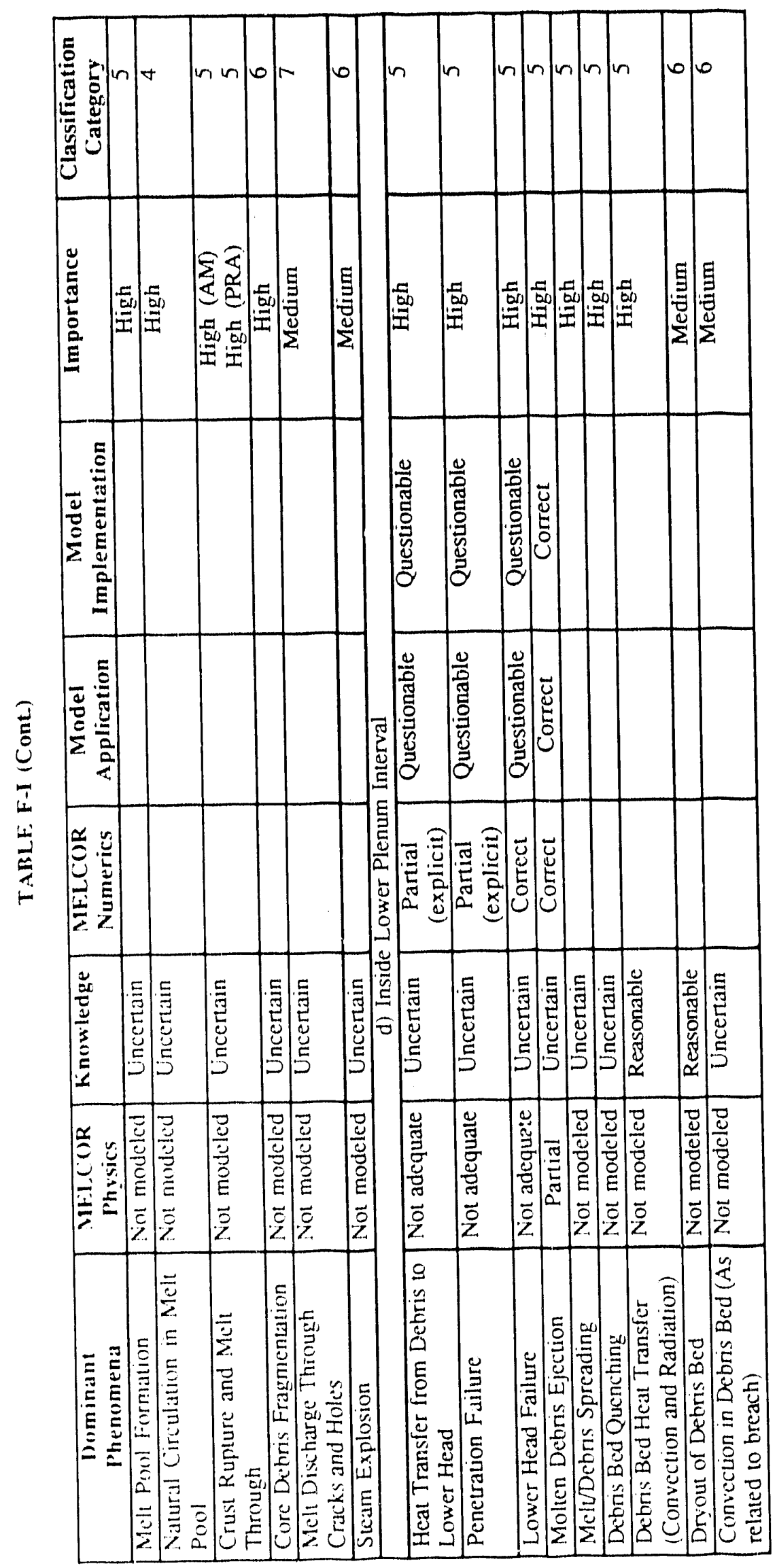


(2) Calculated results may be sensitive to the nodalization (lumped-parameter size) and the time"step increment.

(3) Some of the models in the COR package employ user-spectfied muximum mass/heat-transfer rates to mitigate the numerical instabilities caused by sudden events. However, these specified rates have no physical basis and appear to be used because they work and mitgate the problem.

(4) Sensitivity studies based on different nodalization schemes and time increments have not been performed to insure the convergence of the calculated results.

\section{F.3. RESULTS OF PARAMETER AND SENSITIVITY STUDIES}

Parameter and sensitivity studies specifically aimed at assessing the COR package are nonexistent. The sensitivity studies that have been performed are either as a part of the experimental benchmarking activities or full-plant thermal-hydraulics and source term simulations. Two references report some sensitivity calculations at the code level (Refs. F-1 and F-2), A number of sensitivity calculations have been performed as part of the MELCOR posttest calculation of HDR experiments (Ref, F-2). Time increment, control volume nodalization, and other sensitivities have also been performed as a part of the validation exercise for the BMC-F2 experiment (Ref, F-1).

\section{F.4. RESULTS OF BENCHMARKING/VALIDATION ACTIVITIES}

The need for MELCOR benchmarking and validation has been recognized by the conde developers (Ref. F-3). Unfortunately, no benchmarking/validation activities specifically aimed at the COR pactage or the models in the package could be identified. Some benchmarking and validation tests at the MELCOR code level that impact the COR package have been made and are discussed in this section.

The treatment of physico-chemical phenomena during in-vessel core-melt progression require the input of correct physical/chemical parameters and appropriate use of validated models. The MELCOR code is being developed to extrapolate from a limited number of small-scale tests (TMI-2 accident excluded) to the large-scale core-melt scenario. Code verification and validation through comparison against known physical behavior would be most useful for increasing our understanding of the complex phenomena and to judge the ability of MELCOR to model core-melt progression in a LWR. Unfortunately, validation of the COR package is extremely limited. Hence, this state of code development leaves many important accident progression questions unresolved and certainly has not yielded a high level of confidence in understanding of the phenomenology.

Only two benchmarking and validation tests have been simulated using MELCOR Version 1.8.0 (Refs. F-4 and F-5). Recently, Madni (Ref, F-5) has reported use of the code to simulate the Power Burst Facility (PBF) Severe Fuel Damage (SFD) Test 1-4. The simulation revealed that there were many uncertainties in the performance of the test as well as several model uncertainties and simplifications in the code. In spite of this, the MELCOR Version 1.8.() F-12 simulations have represented the bundle behavior reasonably well. Results of predictions for the transient liquid level in the test bundle, clad temperatures, shroud temperatures, clad oxidation and hydrogen generation, bundle geometry changes, fission product release, and heat transfer to the bypass flow have been made and compared with test data as well as SCDAP/RELAP5 calculations. This benchmarking exercise lends credibility to MELCOR as well as identifies its limitations in predicting events and conditions associated with the early phase of severe core damage. The above findings are reinforced by the results of the simulation of the PHEBUS Severe Fuel Damage Experiment B9+ (Ref. F-4). The comparisons of the thermal behavior of the bundle during high 
fission power henting and oxidation phases show good correspondence with the lest data, but only after an unusual degree of "fine tuning" of the model purameters. The sensitivity calculations revealed several code weaknesses and errors in order to Improve comparisons of thermal behavior with data. These calculations form a good basis for assessing core-melt progression models in the COR package. The comparisons of the core-melt progression and degradation phenomena measured in the PHEBUS B9+ test and calculated by other computer codes involved in the ISP.28 program will provide another opportunlty to assess MELCOR's parametric approuch in modeling core degradation and relocation phenomena. The preliminary conciusions of the program emphasize the great sensitivity in predicting melt behavior to such parameters as oxide shell breach criteria, the need for more extensive validation, and the needed skill and knowledge on the part of code users.

Data are not yet available to assess several of the submodels in the COR package, particularly for the later stages of core-melt progression after the loss of the initial core geometry. Examples of such submodels include core relocation (slumping); "low blockage, lower head and penetration attack, and others. It is not likely that needed test data will become available in the foreseeuble future. Therefore, it is essentlal that the parametric models of phenomena in MELCOR be compared and benchmarked against more ditalled mechanistic codes such as SCDAP/RELAP5 and/or others. Unfortunately, the "two-tier" appronch in modeling severe nuclear reactor accidents adopted by NRC has not been implemented. This has impacted MELCOR in a number of ways, including the types of sensitivity studies and benchmarking that may be possible, level of detall of models that should be included in the code, and the intended uses of the coode.

\section{F.5. IDENTIFIED DEFICIENCIES AND PLANNED IMPROVEMENTS}

A summary of COR package deficiencies is provided in Table F-I and some of these will be discussed here. A number of MELCOR 1.8.0 shortcomings have also been identified by the code developers (Ref. F-6), and some of these overlap with those given in the table.

The complexity of the physico-chemical and radiological phenomena that determine the scenarios for severe accidents in nuclear plants are well recognized. Many of these phenomena related to the COR package are identified in Table F-1. Several of the perceived modeling shortcomings in MELCOR are discussed below. Suffice it to emphasize here that phenomenological uncertainties and lack of data play a very important role in the simulation of a severe accident progression and in its quantification.

The modeling of complex physico-chemical phenomena in core-melt progression, relocation, and lower head attack is not well conceived. Too much emphasis has been placed on modeling the phenomena parametrically and not enough on using such classical methods of scaling analysis, zero-order estimates, and ideal model simulation. Also, there does not appear to have been sufficient interaction of code developers (code architects, phenomenologists) and experimentalists as well as other modelers in the severe nuclear reactor accident community both within and outside SNL. The experimental and test data, both from within and especially from outside NRC sponsorship, have not been factored into the COR package of the code. As a concrete example, one can mention the core materials interactions and behavior that would affect eutectic formation and liquefaction and impact greatly the core-melt progression and possibly timing of the lower head or penetration failure (Refs. F-7 and F-8). As a conseguence, some submodels of the package are not state of the art.

Many important phenomena related to core melting, relocation, and lower head altack have been modeled (Table F.I ) and others are treated parametrically, but no analysis or methodology is provided for estimating the critical parameters. Admittedly, some phenomenological issues related to core-melt progression are not well enough understood, which can lead to speculation, but the code developers are "experts" and should provide some guidance to the user on how to exercise "engineering judgment" and estimate the parameters based on scaling studies, fundamental and mechanistic experiments, and integral analyses. Some phenomena could have been better treated using zero-order estimates than parametrically. 
Detailed modeling of the core meltdown and relocation process is very important in the accident progression. These in-vessel processes determine the initial conditions for the analysis of ex-vessel core-melt progression phenomena and dominate the source term release for early containment failure. Distinct temperature regimes exist in which solid and/or liquid core material relocation processes will have a decisive influence on core heatup, slumping, blockage formation, time-dependent and overall hydrogen generation, possible steam explosion, fission product release, and the chemical composition and temperature of the core debris at the time of the reactor pressure vessel failure. The eutectic interaction and liquefaction of high-temperature core materials far below their melting points and their premature relocation must be considered. The shortcomings of the core materials interaction model are recognized by the MELCOR code developers (Ref. F-9).

Zircalloy melting and relocation can eventually result in slumping in water below the lower support plate and into the lower head of the reactor pressure vessel. During this relocation, the hot material can generate significant additional steam during the quenching process, which can further oxidize materials in the hot core. In addition, it is possible for hydrogen to be generated in this configuration by the oxidation of some of the unoxidized zirconium or other materials in this melt. Quenching of the relocated core material, dryout of the debris bed, reheat of the debris bed, and melt propagation in the dry debris bed are not modeled in the COR package. The postdryout meltdown of such debris bed and the growth of the melt pool will impact the thermal-mechanical attack of the lower head and the vessel failure.

Ballooning is a critical phenomena as it significantly affects early phase melt progress in low pressure experiments such as LOFT LP-FP-2, PBFSFD 1.4, CORA-15, and the TMI-2 reactor. In TMI-2 the blockage formed by solidifying materials aided in forming a melt pool, and after the crust failed, the melt from the pool spilled into the lower plenum. The COR package does not allow for a solid metal layer formation on the lower head, which has occurred in TMI- 2 and protected the head from the hot debris. In this respect, MELCOR is not capable of simulating a single prototypic experiment, albeit unplanned (TMI-2), as far as the phenomena in the lower head is concerned.

Currently no model exists in MELCOR to treat in-vessel and countercurrent natural circulation. This is particularly critical for PWRs, and the code developers have recognized the need (Ref. F-9). The current dT/dz model for calculating local fluid temperatures in the core and lower plenum is not capable of treating natural circulation. This missing model may particularly impact the creep rupture failure of the hot leg, the surge line, and the thermal-mechanical attack of the lower head and penetrations. A model for natural convection in molten pools is also missing.

During a core-melt accident, particularly occurring in the reactor vessel of a Mark-1-type boiling water reactor, there is a potential for failure of the reactor vessel stainless steel liner resulting from thermal attack by core melt. A steam explosion phenomenon could occur during the contact of a hot molten metal with a cold vaporizable liquid. But this type of physical phenomenon is not modeled in the COR package. During a steam explosion, the thermal energy of metal is converted into the potential energy of liquid and vapor mixture with rapid vapor generation. The resulting mixture expands against the surroundings, incurring physical damage to the structures. Because of the special features of the design, a possibility exists in LWRs for the occurrence of steam explosions during severe core-melt accidents.

The lower head model has a number of shortcomings, some of which have been recognized by the MELCOR code developers (Refs. F-6 and F-9). Included in the list of the missing models are the radial debris spreading and melting, lower head curvature, and radial heat transfer.

MELCOR improvement needs have also been assessed and documented (Ref. F-9). Some specific improvements, which have been planned for the COR package, include in-vessel natural circulation, core materials interaction, lower plenum debris modeling, partially submerged component heat transfer, debris bed heat transfer, and radiation heat exchange enhancement. The first two items listed have received highest priority and will probably be completed first, but at the present time it is not clear when this will be accomplished. 


\section{F.6. SUMMARY OF FINDINGS}

\section{F.6.1. General Observations}

Our current knowledge with respect to a number of key phenomenological issues related to ir:-vessel core-melt progression, core relocation, lower head attack, and breach are not reflected in the COR package of the MELCOR code. Some general observations follow about the process of codie development and the code itself.

- Greater emphasis has been placed on MELCOR code architecture, numerical methods and techniques (i.e., develop fast-running code), and use of imported codes than on description of physical phenomena for which models were lacking.

- Traditional approaches of studying complex physico-chemical phenomena (i.e., scaling, dimensionless, and zero-order analyses) were not applied to enable simplifications in the theoretical analysis in developing mathematical descriptions of the phenomena.

- Too much emphasis has been placed on parametric modeling of some physical phenomena and not enough on using classical methods such as scaling analysis, zero-order models, and ideal simulations in developing models for the COR package.

- $\quad$ Some physical processes that could have been treated phenomenologically are treated parametrically, and very little or no guidance is provided to the user for selecting a reasonable physical range of "user-specified" parameters. This could have been accomplished employing zero-order analyses to guide the user.

Although parametric models can be used as surrogates for phenomenological models, such use requires knowledge of timing of accident progression and related thermal-hydraulic conditions. Benchmarking of such parametric models is clearly highly desirable. Unfortunately, the individual models in the COR package have received little or no validation and benchmarking. Parametric models should not be used as a substitute for mechanistic and phenomenological models. Mechanistic "zero-order," "best-estimate," and phenomenological models can be validated as test data become available and, if needed, can be improved. Such descriptions are scrutable by a peer review and are preferable over a parametric approach. Some models in the COR package seem to represent not the physics but the code developer's view of the accident progression.

Detailed modeling of the core-melt progression processes is very important. Yet, a number of key issues have not been considered in developing the code. The data base used in the development of the core-melt progression does not consider the available results of the last several years. The models used in the core-melt progression and lower head attack and breach have no record of validation or peer review. The parametric models are also deficient in areas such as time resolution and dynamics.

Lastly, a number of assumptions and idealizations have been made, but were not clearly stated or successfully articulated in the documents. These overall shortcomings must be overcome if the MELCOR code is to gain acceptance as a credible severe nuclear reactor analysis tool intended by NRC.

In summary, it should be stated that understanding of the core-melt progression phenomena involves a formidable array of requirements, e.g., failure analysis of structural materials at elevated temperatures, description of liquification processes in multicomponent mixtures, modeling of two-phase flows, prediction of gas-side flow patterns and flow rates when the core is partially blocked, chemistry of interactions of different materials at elevated temperatures, and specification of a host of property data to input into the models forming the COR 
package. Unfortunately, there is a dearth of applicable data, and current descriptions of the coremeltdown behavior require validation, because one can model only effects that one is aware of.

\section{F.6.2. Detailed Findings and Relationships to Dominant Phenomena}

Table F-I summarizes the detailed technical findings as related to the dominant severe accident phenomena, together with the characterization of technical adequacy findings. An assessment of the relative importance of the various physical phenomena is also provided. Detailed technical findings related to individual COR package models are provided in Section F.7.

\section{F.6.3. Summary List of Category 4 and 5 Models}

The Category 4 and 5 models in the order of priority are judged to be the following.

(1) PWR primary system natural circulation.

(2) Melt pool phenomena in core (formation, natural circulation, crust rupture).

(3) Cladding ballooning (blockage, flow diversion, double-sided oxidation).

(4) Core reflood phenomena (quench-induced shattering, excess $\mathrm{H}_{2}$ production).

(5) Melting and refreezing-candling models (including eutectic formation and chemical interactions).

(6) Energetic fuel-coolant interactions.

(7) Flow blockage (rod spacers, relocation and slump, core plate).

(8) Melt debris spreading and debris bed heat transfer (including quenching, convection and radiation, dryout).

(9) Heat transfer to lower head and penetrations (including melting of debris, natural convection in melt pool) and lower head and penetration failure.

\section{F.7. FINDINGS FOR INDIVIDUAL MODELS INCLUDED IN MELCOR}

The following pages are summaries of the findings for the individual models appearing in the COR phenomenological package. 


\section{MODEL TITLE: SINGLE-PHASE CONVECTION HEAT TRANSFER}

\section{Model Description and Pedigree:}

Empirical correlations for well-characterized and defined laminar and turbulent steadystate flows given in textbooks are used to calculate spatially averaged convective heat-transfer coefficients. Strictly speaking, the correlations used are appropriate for steady-state or quasisteady conditions and are not appropriate for predicting heat-transfer coefficients under rapid transients. There appears to be no clear distinction when forced and free convection correlations should be used.

\section{Implementation Within MELCOR:}

Empirical constants used in both laminar and turbulent free and forced convection correlation are given; however, the characteristic lengths needed to define the dimensionless parameters are not clearly defined. For example, in Eq. (2.3.6) of Ref. F-10, what characteristic length, fuel rod diameter or some type of hydraulic radius should be used to define the Nusselt number $(\mathrm{Nu})$ and the Reynolds number $(\mathrm{Re})$ ? In spite of the fact that mixed convection could occur, only correlations for free and forced convection are given.

\section{Results of Model and Parametric Sensitivity Studies:}

No such results were found. The empirical constants appearing in most correlations used are implemented in sensitivity coefficients that can be input by the user.

\section{Results of Model Benchmark/Validation Activities:}

The correlations are based on steady-state data obtained under the reported experimental conditions and range of parameters. Benchmarking the correlations for geometries, different than those for which they were originally developed or for rapid transient processes, has not been performed.

\section{Identified Deficiencies and Planned Improvements:}

No rationale is given for using steady-state correlations under quasi-steady or transient conditions. The boundary conditions and geometrical configurations to which the correlations are applied may not be exactly the same as those in the experiments. For example, Eqs. (2.3.9) and (2.3.10) of Ref. F-10 are not appropriate for predicting the heat-transfer coefficient between a debris bed and a fluid stream. The correlation is valid for an isolated spherical particle and the fluid. This deficiency is recognized by the code developers, and the correlation will be replaced in the future, but it is not clear why appropriate correlations were not used in the first place.

\section{Summary of Findings:} predictions.

Application of the correlations to rapid transients is expected to lead to inaccurate 


\section{Importance Assessment:}

Convection is an important phenomena, particularly at lower component temperatures and before core uncovering, and it can play an important role in timing of such events as the start of Zircalloy oxidation.

\section{Technical Adequacy:}

Intact core components: Category 1.

Degraded core components: Category 3. 


\section{MODEL TITLE: BOILING HEAT TRANSFER}

\section{Model Description and Pedigree:}

Only saturated pool boiling is considered and flow boiling is not implemented in the code. The correlation used is dated (1950s) and does not reflect the research done during the last 40 years. Nucleate and film boiling regimes are demarcated by an arbitrarily specified temperature superheat. A radiation component is added to the convective heat- transfer coefficient for film boiling. The expression is reasonable, but an arbitrary value of 0.4 is "hard-coded" for the emissivity, and no physical justification is provided for using this value. Boiling heat transfer from particulate debris is not treated.

\section{Implementation Within MELCOR:}

Constants in the empirical heat-transfer coefficient correlations have been implemented as sensitivity coefficients. According to the COR Package Reference Manual (page COR-MM-33 of Ref. F-10), transition boiling is not treated, whereas the COR Package User's Guide (page CORUG-45 of Ref. F-10) suggests that it is treated approximately. This apparent inconsistency needs to be clarified.

\section{Results of Model and Sensitivity Studies:}

None found.

\section{Results of Model Benchmark/Validation Activities:}

None identified.

\section{Identified Deficiencies and Planned Improvements:}

The temperature superheat separating nucleate and film boiling regime is not a constant and depends on the material-liquid combination, but this is not reflected in the code. Also, the emissivity of the solid in the expression for the radiation heat-transfer coefficient for film boiling should depend on the material. In addition, the emissivity is expected to change as the material reacts, but chemical reactions of core materials are not considered during film boiling. The empirical correlations for nucleate and film boiling heat-transfer coefficients [Eqs. (2.3.11) and (2.3.12) on page COR-RM-33 of Ref. F-10] are not appropriate for particulate debris, and there is no discussion in the documentation of how boiling heat transfer from the debris to water should be predicted.

\section{Summary of Findings:}

Correlations well documented in the literature have been used, and the treatment is at a reasonable level, but the documentation is not adequate.

\section{Importance Assessment:}

Deficiencies may lead to inaccuracy in the timing of events, Zircalloy oxidation, hydrogen generation, and the physical state of the degraded core. 


\section{Technical Adequacy:}

Intact core components: Category 1.

Degraded core components: Category 3. 


\section{MODEL TITLE: CONTROL VOLUME TEMPERATURE DISTRIBUTION (dT/dz) MODEL}

\section{Model Description and Pedigree:}

The $\mathrm{dT} / \mathrm{dz}$ model is based on an energy conservation principle for the gas contained in the control volume. Unfortunately, the basic equations of the model, Equations (2.5.2) and (2.5.3) of Ref. F-10, are in error if the specific heat of the gas is considered to be a function of temperature, as stated in Ref. F-11 (pages 4-6). The model uses the approximation of steady unidirectional (upwards) flow of gas through a channel with a known gas inlet temperature. The gas flow, strictly speaking, is one dimensional, and no cross flow between core rings is allowed. Hence, this idealization restricts application of the model to truly one-dimensional flows, and multidimensional flows such as would be expected under natural circulation conditions in the RCS cannot be handled. The model appears to neglect radiation heat transfer from the surface to the coolant (steam-hydrogen) mixture as there is no discussion of how the radiation contribution is to be calculated. It has been established that at high temperatures and low coolant flow rates, the radiation heat-transfer rate in the core may be much larger than the convection heat-transfer rate.

\section{Implementation Within MELCOR:}

Implementation of the model in MELCOR is appropriate and numerical methods are consistent with the control volume formulation. The CF package provides some flexibility in handling more realistic flows, but the statement "... thus allowing the user to do anything he wants..." (Ref. F-10, page COR-RM-40) does not appear to be appropriate without some caveats.

\section{Results of Model and Sensitivity Studies:}

None identified.

\section{Results of Model Benchmark/Validation Activities:}

None found.

\section{Identified Deficiencies and Planned Improvements:}

Some deficiencies of the $\mathrm{dT} / \mathrm{dz}$ model have been identified by the MELCOR code developers (Refs. F-9 and F-11) and the deficiencies are attributed to the one-dimensional flow assumption as well as to overlapping jurisdiction with the $\mathrm{CVH}$ package. The model has been found to behave poorly when the one-dimensional (upflow) assumption is violated. Also, the documentation is not as clear and unambiguous as it should be. For example, in Ref. F-11 (p. 67, paragraph 1) it is stated that "MELCOR can currently model such natural circulation coarsely with the Control Volume Hydrodynamics (CVH) package (although without the momentum flux terms)." However, two paragraphs later it is stated that "Because flow patterns other than upflow in the core region proper violate the assumptions of the current $\mathrm{dT} / \mathrm{dz}$ model, it is inappropriate for treatment of natural circulation." Some improvements of the dT/dz model are planned (Ref. F-9), but the nature of the model is such that the deficiencies cannot be easily remedied without major restructuring of the code to enable handling multidimensional flows. 


\section{Summary of Findings:}

The model equations used are incorrect and need to be corrected and the code revised. For one-dimensional upflow the model is reasonable, but after the core degrades and partial or total blockages occur, the flow will be multidimensional and the model will fail to predict accurately the gas temperature along the core. Heatup or cooldown of core and internal structures may not be calculated accurately.

\section{Technical Adequacy:}

Intact core components (i.e., one-dimensional flow): Category 4.

Degraded core components (i.e., partially blocked flow): Category 4. 


\section{MODEL TITLE: FISSION POWER GENERATION MODEL}

\section{Model Description and Pedigree:}

In addition to the decay heat power (handled in the DCH package), the COR package contains a simple model that calculates the fission power either directly or from two different userdefined functions. The heat generated in the fission power model is distributed over the core cells using the radial and axial relative power densities prescribed by the user on input.

\section{Implementation Within MELCOR:}

The user has the flexibility of using an empirical correlation or user-defined function to calculate fission power generation. The constants in the correlation are implemented as sensitivity coefficients.

Results of Model and Parameter Sensitivity Studies:

None identified.

Results of Model Benchmarking/Validation Studies:

None found.

\section{Identified Deficiencies and Planned Improvements:}

Gamma heating of core structures is not calculated, but may be important in interstitial regions (i.e., control blades) of BWRs (Ref. F-11). For ATWS accident sequences, the existing fission power correlation applies only to BWRs with saturated fluid inlet conditions to the core (Ref. F-11) and a correlation for PWRs is needed.

\section{Summary of Findings:}

The basic approach is physically reasonable. The model is not likely to be a limiting factor in the predictions of the COR package.

Technical Adequacy:

Category 1. 


\section{MODEL TITLE: GRAVITATIONAL SETTLING}

\section{Model Description and Pedigree:}

Downward relocation of particulate debris by gravitational settling is modeled not mechanistically but by a logical sequence of processes through consideration of volume, porosity, and support constraints. Relocation of relatively intact components can also be treated whenever lower components, which normally provide support, are eliminated by melting or failure. Radial movement of debris particles is not allowed.

\section{Implementation Within MELCOR:}

The gravitational settling is implemented by a set of user-specified input parameters (i.e., porosity, interstitial volume, failure temperature, etc.). Relocation of particulate debris and of intact components is treated differently.

\section{Results of Model and Parametric Sensitivity Studies:}

None found.

\section{Results of Model Benchmarking/Validation Activities:}

None identified.

\section{Identified Deficiencies and Planned Improvements:}

The current limitation of the computational scheme is that no distinction is made between failure mechanisms for different types of structures or varying mechanical loadings. Relocation is governed only by user-specified failure temperature.

\section{Summary of Findings:}

The current modeling is simplistic, but the processes considered are complex and empirical information is lacking for improving the treatment. In view of the empirical nature of the modeling, validation and guidance on reasonable ranges for the input parameters are neede $\mathbb{A}$.

\section{Importance Assessment:}

This is an important phenomena that can give rise to core blockage and to large changes in core geometry.

\section{Technical Adequacy:}

Category 2. 


\section{MODEL TITLE: LOWER HEAD HEAT TRANSFER}

\section{Model Description and Pedigree:}

Many of the phenomena associated with the lower head failure are poorly understood, and heat transfer from the debris to the lower head and its penetrations is modeled parametrically with user-specified heat-transfer coefficients. The lower head model is essentially one dimensional. Lateral movement of debris across radial ring boundaries is not modeled, and curvature of the head in communicating thermally with the debris or heat transfer between radial rings and the vessel wall cylinder is not considered. The debris bed communicates thermally with the inner surface of the lower head by transferring heat; however, the coupling is not mechanistic but parametric. There are no validated mechanistic heat-transfer models from the debris bed to the lower head.

\section{Implementation Within MELCOR:}

The model is implemented parametrically by user-specified heat-transfer coefficients and heat-transfer areas and masses. No logic or numerical inconsistencies have been identified.

\section{Results of Model and Parametric Sensitivity Studies:}

None identified.

Results of Model Benchmarking/Validation Activities:

None found.

\section{Identified Deficiencies and Planned Improvements:}

The lower head heat-transfer model is considered to be inadequate. There is no guidance provided in the documentation of how to estimate the heat-transfer coefficient between the debris bed and the penetrations or the low'er head. Default values of these coefficients are given in Ref. F10 (page COR-UG-15), but these coefficients (constant values) appear to be completely arbitrary.

\section{Summary of Findings:}

Validation and theoretical justification on the choice of model parameters are needed to provide some confidence in the simplified parametric model used. Uncertainties in the heat-transfer coefficient between the bed and penetrations or lower head limit the accuracy of the model and timing of events.

\section{Importance Assessment:}

Lower head heat transfer is a dominant phenomena that impacts greatly on the timing of the pressure vessel failure, fission product release into the containment, and pressurization of the containment, so confidence in the model is critical.

\section{Technical Adequacy:}

Category 5. 


\section{MODEL TITLE: LOWER HEAD FAILURE}

\section{Model Description and Pedigree:}

Failure of the lower head is handled parametrically. The head is assumed to fail and produce an opening with an initial diameter specified by the user whenever the temperature of the penetration or innermost lower head node reaches a failure temperature specified by the user. Mechanical loading of the head is not considered in the failure mode.

\section{Implementation Within MELCOR:}

The implementation is technically sound.

\section{Results of Model and Parameter Sensitivity Studies:}

Nore foind.

\section{Results of Model Benchmarking/Validation Activities:}

None identified.

\section{Identified Deficiencies and Planned Improvements:}

The lower head failure model is perceived to be inadequate (Ref. F-6). A credible predictive model for lower head structural failure would require a finer subdivision into finite difference nodes with a temperature distribution computed from a conduction solution and failure based on thermal-mechanical criteria.

\section{Summary of Findings:}

The current model is simplistic and requires improvement. There is a need for validation and guidance of the user on reasonable ranges for the failure temperature and of the initial opening diameter. This is particularly important because of the empirical nature of the modeling and the fact that mechanistic models are lacking.

\section{Importance Assessment:}

Lower head breach is a critical phenomenon. It impacts timing of depressurization of the reactor vessel, fission product release into the containment building, and survival of the containment. There is a critical need for an adequate model.

\section{Technical Adequacy:}

Category 5. 


\section{MODEL TITLE: DEBRIS EJECTION}

\section{Model Description and Pedigree:}

Upon failure, molten mass is ejected through the opening of a penetration at a rate calculated from the Bernoulli equation by specifying the pressure difference, flow area, debris height, and user-specified loss coefficient. Ablation of the failure opening is modeled by calculating the heat-transfer rate to the lower head frcm the molten debris. In spite that this is a highly transient process, the heat-transfer coefficients between the molten debris and the penetration opening (i.e., tube or plate) is determined from steady-state empirical correlations for turbulent flow. Also, whenever the buttom lower head node exceeds the penetration failure temperature, gross failure of the lower head in that ring is assumed, and all debris in the bottom cell is discharged immediately, regardless of the failure opening diameter or the time step. The model also does not account for erosion of the opening by the debris.

\section{Implementation Within MELCOR:}

The implementation is technically sound. There is enough flexibility provided to the user in exercising the code through some simple options. Mass and energy ejected from the COR package is transferred to the transfer process (TP) package, and the CAV, FDI, and RN packages can then interact with TP.

\section{Results of Model and Parameter Sensitivity Studies:}

None identified.

\section{Results of Model Benchmarking/Validation Studies:}

N.one identified.

\section{Identified Deficiencies and Planned Improvements:}

Equations (4.3.3) and (4.3.4) of Ref. F-10 (page COR-RM-65) used to predict the ablation rate of the failure opening are well-known equations for steady-state conditions. They are not appropriate for rapid transients. No physical justification is provided for using the equations in this context, not only because they have been obtained for steady heat transfer but also because they are not valid for liquid metals. At this stage of a severe accident, one would expect the debris to be composed primarily of molten metals.

\section{Summary of Findings:}

On its own, the model is reasonable and the treatment is adequate. Unfortunately, it is intimately coupled to a lower head failure model. As a consequence, the model can lead to inaccurate predictions. There is a need for model validation.

\section{Importance Assessment:}

Contribution to the uncertainty in the final results is not expected to be very significant.

\section{Technical Adequacy:}

Category 5. 


\section{MODEL TITLE: CLAD BALLOONING}

\section{Model Description and Pedigree:}

There is no comprehensive model for clad ballooning in the code, although sor:e of the effects of ballooning can be simulated to a limited extent. The cladding failure temperature is userdefined (input to the RN package); this parameter controls the release of fission products from the pellet/cladding gap. Flow restrictions from ballo oning may be simulated in a limited way in $\mathrm{CVH}$ using control functions to reduce flow areas, but the effects would not be totally translated into the COR dT/dz convective heat-transfer model (Ref. F-12). is provided.

No treatment of flow diversion, inner cladding surface oxidation, or strain cooling effects

\section{Implementation Within MELCOR:}

There is no model implemented in the code.

\section{Results of Model and Parameter Sensitivity Studies:} found.

No model or parametric sensitivity studies on the limited facilities available have been

\section{Results of Model Benchmarking/Validation Activities:}

The default clad rupture temperatura, is in reasonable agreement with observation (Refs. F-13 and F-14).

\section{Identified Deficiencies and Planned Improvements:} documentation.

No plans for including a more detailed model have been identified in current SNL

\section{Summary of Findings:}

A more comprehensive treatment of the effects of ballooning is recommended, covering the effects of flow diversion, strain cooling, and increased oxidation caused by exposure of the inner surface of the cladding. It is judged that the totality of these effects cannot be adequately simulated by varying existing input parameters.

\section{Importance Assessment:}

Ballooning is of medium importance in low-pressure transients. There are effects on thermal-hydraulics, heat transfer, oxidation, and fission product release that are important in an accident management context. The phenomenon does not occur in high-pressure transients and is judged unimportant in a PRA context.

\section{Technical Adequacy:}

Low-pressure sequences (AM): Category 4.

Other cases: Category 7. 


\section{MODEL TITLE: CANDLING}

\section{Model Description and Pedigree:}

The melt flow model is thermal-hydraulically based and does not take into account the material properties (viscosity, surface tension, etc.) that may control the velocity of the melt flow. Each material is considered separately. in each time step, if molten material has been generated at some location in the core, this material is assumed to have been generated at a constant rate and to have flowed downwards ove: the component surfaces in lower cells. The amount of material solidifying in each cell is determined by calculating the energy transferred to the given cell component, accounting for any cooldown of the molten material to its melting point, and dividing the resultant energy transfer by the latent heat of fusion. No allowance is made for the transit time of the material, which is assumed to be small. Heat transfer between the melt and the underlying intact component is controlled by "refreezing heat-transfer coefficients" whose values may be altered to simulate different assumed flow configurations. A similar approach has been followed in the BWRSAR code developed at Oak Ridge National Laboratory.

Blockages are allowed to form when the refrozen material completely fills a cell, and molten pools can form. There is a simple model for the holdup of melt inside an oxide shell, based on a minimum oxide thickness and maximum temperature for the shell not to fail, for zirconium and steel oxides. The break-out flow from a fractured shell (or from a failed blockage) can be limited by user parameters.

There is an empirical model to deal with transport of secondary materials, such as $\mathrm{UO}_{2}$ or $\mathrm{ZrO}_{2}$ by the candling processes. The secondary transport is specified either as an input fraction of the molten mass or as a proportion of the existing fraction in the component for a given material. It does not treat the chemical/eutectic reactions involving core materials; these are reviewed separately.

The model is described in Refs. F-10 and F-11 with additional material in Refs. F-12, F15 , and F-16.

\section{Implementation Within MELCOR:}

Relocation is calculated in each time step after the new temperature distribution has been determined. There are user inputs for the refreezing heat-transfer coefficients for each material, for the control of transport of secondary materials during candling, and parameters to control the holdup of molten materials by oxide shells and the break-out flow once such an oxide shell has been breached. Although defaults are given, no basis for these is provided in the user's guide on how to select appropriate values of the coefficients.

\section{Results of Model and Parameter Sensitivity Studies:}

Parametric studies of the effects of varying refreezing heat-transfer coefficients have been discussed by Madni (Ref. F-14) in connection with analysis of PBF SFD tests and plant studies. Default values are more 'opropriate to film flow (not observed in experiments); lower values (e.g., by a factor of 3 ) that give vetter agreement with data are more consistent with the observed rivulet flow. Madni also notes a strong dependence of the amount of melt relocated on the holdup parameters, which is consistent with the reviewer's own experience with SCDAP/RELAP5.

\section{Results of Model Benchmarking/Validation Activities:}

There has been as yet no direct separate-effects validation of the candling models (Ref. F15). It is ciifficult in integral experiments to factor out the effects due to the candling model alone. However, the ISP-28 calculation of PHEBUS B9+ (Ref. F-4) by SNL gives the opportunity to give validation by comparing the end state blockage fractions and relocated melt compositions 
calculated by the code with the experimental results; this has been recognized by the MELCOR team (Ref. F-16). An initial comparison of the MELCOR blockage profile with the experimental cata shows melt formation predicted above the experimentally observed region with a debris bed at the top of the bundle (not observed in the experiment); refreezing is, however, calculated at the observed location. Under the conditions of this experiment, the melt therefore was calculated to relocate too far.

\section{Identified Deficiencies and Planned Improvements:}

One main deficiency in the relocation area concerns eutectic formation and its effect on relocation; this has been assessed separately. More guidance is needed on the choice of refreezing heat-transfer coefficients, because the defaults are not necessarily the best choice (Ref. F-14) and were not used in the ISP-28 submission (Ref. F-4). This is important because the answers obtained can be very sensitive to the choice of refreezing heat-transfer coefficients used; these are very uncertain parameters. Similar comments apply tc the oxide shell breach criteria. Comprehensive benchmarking of the model is particularly necessary in view of its highly parametric nature; the current level is inadequate.

\section{Summary of Findings:}

More justification, both theoretical and by validation, is needed to provide confidence in the simplified parametric model used. Uncertainties in refreezing heat-transfer coefficients and oxide shell failure criteria limit the reliability of the model output. The uncertainties in the modeling are more important in accident management, where issues of timing are more significant, than in PRA applications. The categorization of this model takes into account lack of benchmarking and the input parameter uncertainty.

\section{Importance Assessment:}

Candling of molten mattrial is a dominant phenomenon that has an important effect on the final core state; confidence in the model is essential particularly for accident management applications.

\section{Technical Adequacy:}

Category 3 (PRA).

Category 5 (AM). 


\section{MODEL TITLE: CONDUCTION}

\section{Model Description and Pedigree:}

Axial conduction between cells is modeled separately for each component, while radial conduction is calculated across fuel/cladding gaps (which have a constant, user-defined size; differential thermal expansion of fuel and cladding is neglected). Other than that, a lumpedparameter approach is adopted within each cell, and a quasi-steady-state temperature profile, which is parabolic for fuel, is assumed. The thermal resistance of fuel rod cladding is neglected. An approximate analytical model for axial conduction is used at the liquid level interface, where axial temperature gradients can be large.

No mention of any modification to the geometry, etc., when material freezes on the cladding surface, can be found in the code description. The modeling is described in Refs. F-10 and $\mathrm{F}-11$.

\section{Implementation Within MELCOR:}

The COR package uses an explicit integration scheme for time advancement, with a subcycling capability so that several time steps can be taken internally by COR within a single system cycle under automatic control. The model for axial conduction between cells is standard finite difference. The cladding gap heat-transfer model is coupled to the cladding convection calculation for stability reasons.

User input is specified for an additional fuel-cladding gap conductance (via a control function), the gap size, and time-step control information (the latter applies to the whole COR package).

\section{Results of Model and Parameter Sensitivity Studies:}

No model or parameter sensitivity studies have been identified.

\section{Results of Model Benchmarking/Validation Activities:}

No model benchmarking or validation studies have been identified.

\section{Identified Deficiencies and Planned Improvements:}

No deficiencies have been identified by SNL. The gap conductance model will need to be modified if a ballooning model is adopted.

\section{Summary of Findings:}

The basic approach is physically reasonable. The modeling of conduction is unlikely to be a limiting factor in the predictions of the COR package.

\section{Importance Assessment:}

Thermal conduction is a fundamental heat-transfer process that needs to be modeled. However, radiation and convection are likely to be more significant for fuel components, at least for severe accident conditions. A possible exception is at the pool interface, where a more detailed model is implemented. 
Technical Adequacy:

Category 1. 


\section{MODEL TITLE: EUTECTIC FORMATION AND EFFECT ON RELOCATION}

\section{Model Description and Pedigree:}

There are no models for eutectic formation in the code (and, therefore, no modeling of the effects of eutectic formation on melt flow).

\section{Identified Deficiencies and Planned Improvements:}

Eutectic formation through reaction between pairs of materials such as $\mathrm{UO}_{2} /$ Zircalloy and Zircalloy/lnconel (spacer grids) lowers the temperature at which melt formation starts and increases the amount of material relocated. The eutectic melt temperatures can be several hundred degrees below tie melt temperature of the individual materials involved.

The need to model eutectic formation ( $\mathrm{U} / \mathrm{Zr} / \mathrm{O}, \mathrm{Zr} / \mathrm{steel}, \mathrm{B}_{4} \mathrm{C} /$ steel) with the consequent effects on promoting material relocation is recognized by SNL (Refs. F-6, F-9, and F-17) and noted in other reviews (Refs. F-18 and F-19). The present review recommends including reactions involving $\mathrm{Ag} / \mathrm{In} / \mathrm{Cd}$ alloy and that between Inconel grid material and Zircalloy. Eutectic rate correlations are now available in many cases (Refs. F-20 through F-24), including the protective effect of oxide films on Zircalloy cladding formed during normal plant operation.

\section{Summary of Findings:}

Models for eutectic formation and the effect on core melt, etc., should be included in MELCOR. This implies a need for data on material properties of eutectic mixtures to be included in the MP package (some of these properties are uncertain).

\section{Importance Assessment:}

This is a phenomenon judged to be important in accident management applications; it will affect the amount of relocated melt and the timing of melt relocation, but is unlikely to affect the final outcome of an accident sequence.

\section{Technical Adequacy:}

Accident management: Category 5.

PRA applications: Category 7. 


\section{MODEL TITLE: METAL OXIDATION AND HYDROGEN PRODUCTION}

\section{Model Description and Pedigree:}

Well-established literature correlations involving parabolic time dependence are used to calculate the oxidation kinetics of Zircalloy and steel. Parabolic kinetics are justified by diffusion theory. A boron carbide oxidation model is included but currently disabled. A model of ratelimiting (gaseous diffusion) blanketing effects is present.

Oxidation is calculated for cladding, canisters, conglomerate debris (frozen films), and debris beds (not treated for structures outside the core region). Account is taken of the shielding of cladding by refrozen material. There is no treatment of double-sided oxidation of fuel rod cladding, which may occur for ballooned cladding in the vicinity of rupture sites. The modeling is described in Refs. F-10 and F-11.

\section{Implementation Within MELCOR:}

No inconsistencies in the implementation have been found. A comprehensive set of sensitivity coefficients is available to vary the kinetics correlations, gaseous diffusion parameters, and the modeling of the surface area of the conglomerate debris and the intact component surface area that is blocked by this debris. The oxidation may also be shut off by control functions to simulate the effect of blockages; cutoff temperatures may also be input. There is no reason to doubt the numerical methods used (analytic integration).

\section{Results of Model and Parameter Sensitivity Studies:}

No model or parameter sensitivity studies have been identified.

\section{Results of Model Benchmarking/Validation Activities:}

Indirect evidence from calculation of the oxidation excursion in early phase melt progression experiments (Refs. F-4 and F-14) shows no deficiency in modeling in the early phase. Hydrogen production is well predicted in steam-starved tests.

Validation of the modeling in steam-rich conditions (e.g., in the LOFT FP-2 experiment) and in later phases of severe accidents (when a debris bed has been formed) is desirable.

Oxidation of Zircalloy and stain'ess steel has been extensively reviewed (Refs. F-25 and F-26) without reference to a particular code for defined intact geometries.

\section{Identified Deficiencies and Planned Improvements:}

Extension to structures outside the core are needed (Ref. F-27), particularly to treat oxidation of steel core components (otherwise total hydrogen generation may be under-estimated). This modeling should be included in the heat structure (HS) package.

Partition of steam under steam-starved conditions should be on an area rather than a hierarchal basis (Ref. F-6).

\section{Summary of Findings:}

Reasonable physical treatment exists with no need for improvement except as given above. Oxidation of debris is more uncertain than oxidation of intact structures. However, in the former case it is judged that there are sufficient user-controlled sensitivity coefficients to bound the expected behavior, though the choice of values will need to be carefully justified by the users. Implementation of the improvements would upgrade the technical adequacy assessments given below. 


\section{Additional Comments:}

Calculation of the excess hydrogen production on quench (for example in LOFT FP-2 and in the CORA tests involving quenching) is still not demonstrably well understood or modeled satisfactorily in any code. This is a matter of modeling excess steam production and/or exposed metal surface area better, rather than the kinetics itself. This difficulty has been noted by SNL also in connection with TMI-2 analysis (Ref. F-28).

\section{Importance Assessment:}

Metal oxidation is an important source of heat and hydrogen production. The treatment in the code for intact geometry is judged to give rise to small uncertainty in source term assessment, but medium uncertainty for degraded geometry.

\section{Technical Adequacy:}

Intact core components: Category 2.

Degraded core components: Category 3. 


\section{MODEL TITLE: PARTICULATE DEBRIS FORMATION}

\section{Model Description and Pedigree:}

A simple model for particulate debris formation is used. Particulate debris is formed when the unoxidized metal thickness of an intact component reaches a user-defined minimum value. It is assumed that the oxidized layers can carry no load and that the metal of the specified thickness and above can support any mass of material above that point; there is no calculation of stress.

When the cladding in a COR cell fails, the fuel and cladding material are converted to particulate debris of a specified particle diameter; the fuel pellets are assunied to have no means of support other than the cladding. The picture is similar for nonfuel components. In the debris, the oxide layers are assumed to reside on the surfaces of their parent metals. If further material arrives, the oxidation and total surface areas are recalculated.

Relocation of intact components is assumed to occur if structures that would normally support them fail. There is no modeling of quench-induced shattering, nor of radial relocation of particulate and molten debris. The modeling is described in Refs. F-10 and F-11 with additional material in Ref. F-16.

\section{Implementation Within MELCOR:}

Minimum cladding thicknesses below which particulate debris is assumed to form are specified for steel and Zircalloy via input data. The average particle diameter and average porosity of particulate debris are also specified in this way.

\section{Results of Model and Parameter Sensitivity Studies:}

No model or parameter sensitivity studies have been identified.

\section{Results of Model Benchmarking/Validation Activities:}

No model benchmarking or validation studies have been identified.

\section{Identified Deficiencies and Planned Improvements:}

SNL accepts shortcomings in this area, including the need to treat quench-induced shattering, lateral movement of debris, and the role of oxidized material in maintaining structural integrity (Refs. F-6, F-11, and F-17), but these are not mentioned in Ref. F-9. No progress was noted at the third review meeting (Ref. F-29).

\section{Summary of Findings:}

The current modeling is simplistic and requires updating in line with the Sandia proposals. More validation and guidance on reasonable ranges for the input data are needed in view of the empirical nature of the modeling. Mechanistic models are lacking in this area.

The absence of a quench-induced shattering model hinders application in reflood scenarios (recovered accidents/accident management).

\section{Importance Assessment:}

This is a dominant phenomenon that can give rise to large changes in core geometry. 


\section{Technical Adequacy:}

Quench situations (AM): Category 5.

Other cases: Category 3. 


\section{MODEL TITLE: RADIATION}

\section{Model Description and Pedigree:}

Thermal radiation among components within COR cells, across cell boundaries, and from components to steam is modeled as exchange of radiation between pairs of surfaces with an intervening grey medium, using the treatment of Kreith (Ref. F-30). Radiative heat transfer between fuel components and the liquid pool (if present) is considered. Radiation exchange factors are user-specified (although defaults are present, these are not recommended; Ref. F-14) and can be changed on restart. Although dynamic adjustment of these factors is not attempted, the disappearance of components is taken into account. Emissivities of steam and surfaces are derived using equations from MARCON 2.1 B. Account is taken of the variation of emissivity of Zirculloy with oxide layer thickness, using a correlation from MATPRO. Approximate representative expressions for beam lengths between components of different types, assuming intact core geometry, are used. The original geometry is assumed throughout the transient, even when a component has relocated or the core is otherwise disrupted.

No account is taken of variation of steel emissivity with oxidation thickness, nor of any difference in emissivity between wet and dry surfaces. Radiative heat transfer between fuel and cladding in fuel components is considered. The radiation modeling is described in Refs. F-10 and F-11, with additional material in Ref. F-15.

\section{Implementation Within MELCOR:}

The user's guide describes input of radiative exchange factors, which "roughly correspond to traditional view factors" (MELGEN input). There is no check if these are physically reasonable (e.g., if summation/reciprocity rules are obeyed). Boundary heit structures must be set up to calculate energy radiated from the COR cells (HS input) and the existence of these is checked by the COR package in MELGEN. At present there must be exactly one heat structure per axial level.

Fuel-cladding gap emissivities may be user-specified. No significant inconsistencies in implementation have been identified.

\section{Results of Model and Parameter Sensitivity Studies:}

No model or parameter sensitivity studies have been identified.

\section{Results of Model Benchmarking/Validation Activities:}

No model benchmarking or validation studies have been identified.

\section{Identified Deficiencies and Planned Improvements:}

View factors can be controlled only on a global basis; a need to be able to control them on a local cell basis has been identified (Ref. F-6). The present review agrees with this assessment.

The present review (Ref. F-15) questions the use of the Ludwig/Ferisso steam emissivities rather than the Cess/Lian empirical equation. 


\section{Summary of Findings:}

The modeling is believed to be adequate and at a suitable level, although the implementation is questionable. The documentation needs improvement, in particular the radiation exchange factors and their relationship to traditional view factors need better definition. It is not a model whose deficiencies limit the reliability of the predictions of the COR package.

\section{Importance Assessment:}

Thermal radiation is the dominant mode of heat transfer at the very high temperatures experienced in the later stages of some postulated severe accidents and, therefore, accurate modeling is important.

\section{Technical Adequacy:}

Category 3.

\section{REFERENCES}

F-1. M. I. Robertson, "MELCOR-A Modeling Overview and Calculations for Phase 1 of the BMC-F2 Experiment," United Kingdom Atomic Energy Authority report PWR/SAWG/P(90)578, PWR/FPERG/P(90)108 (May 1990).

F-2. G. M. Martinez, "MELCOR Posttest Calculations of the HDR Experiment," letter report to R. B. Foulds, US Nuclear Regulatory Commission (September 29, 1989).

F-3. L. N. nmetyk, "MELCOR Assessment Plan," Sandia National Laboratories unnumbered draft (March 1, 1991).

F-4. G. M. Martinez, "MELCOR Calculation of ISP28 SFD PHEBUS Test B9+," Sandia National Laboratories letter report to B. Adroguer (December 14, 1990).

F-5. I. K. Madni, "MELCOR Modeling of the PBF Severe Fuel Damage Test 1-4," Brookhaven National Laboratory report BNL-NUREG-44503 (1990).

F-6. R. M. Summers, "MELCOR 1.8.0 Shortcomings," Sandia National Laboratories unnumbered draft (January 10, 1990).

F-7. P. Hofmann et al., "Reactor Core Materials Interactions at Very High Temperatures," Nuclear Technology 87, pp. 146-186 (Augusi 1989).

F-8. D. K. Akers and R. K. McCardell, "Cor: Materials Inventory and Behavior," Nuclear Technology 87, pp. 214-233 (August 1989).

F-9. R. M. Summers, R. K. Cole, Jr., and E. A. Boucheron, "MELCOR Improvement Needs Assessment," Sandia National Laboratories letter report to R. B. Foulds, US Nuclear Regulatory Commission (October 17, 1990).

F-10, R. M. Summers, "MELCOR Primer, Version 1.8.0," Sandia National Laboratories unnumbered document (February 17, 1989). 
F-11. R. M. Summers, R. K. Cole, Jr., E. A. Boucheron, M. K. Carmel, S. E. Dingman, and J. E. Kelly, "MELCOR 1.8.0: A Computer Code for Severe Nuclear Accident Source Term and Risk Assessment Analysis," Sandia National Laboratories report SAND900364 (NUREG/CR-5531) (January 1991).

F-12. B. E. Boyack, Los Alamos National Laboratory, unpublished questions on "Core Modeling" and "Material Properties," by T. J. Haste, in letter to B. E. Boyack, Regarding: MELCOR Peer Review-Second Meeting, September 27, 1990. Unpublished answers on "Answers to Tim Haste's Questions on COR Package Modeling" by R. M. Summers, Sandia National Laboratories, Second MELCOR Peer Review Meeting, November 5-7, 1990. Unpublished answers on "Responses to Additional Haste Questions on COR Package Modeling," by R. M. Summers, Third MELCOR Peer Review Meeting, January 11, 1991.

F-13. I. R. Madni, "MELCOR Simulation of the PBF Severe Fuel Damage Test 1.1 ," in "Proceedings of the 26th National Heat Transfer Conference," AIChE Symposium Series on Heat Transfer, Philadelphia, Pennsylvania (1989).

F-14. I. K. Madni, "MELCOR 'Lessons Learned': A User Perspective," Brookhaven National Laboratory draft letter report to R. Foulds, US Nuclear Regulatory Commission (January 9, 1991).

F-15. B. E. Boyack, Los Alamos National Laboratory, unpublished questions on "Heat Structures (HS) Package" and "Core Heatup and Degradation (COR) Package," by R. Viskanta, in letter to B. E. Boyack, Regarding: Questions Concerning MELCOR to be addressed by SNL at the November 5-7, 1990 Committee Meeting, September 24, 1990. Unpublished answers on "Heat Structure (HS) Package" by M. K. Carmel, Sandia National Laboratories, Second MELCOR Peer Review Meeting, November 5-7, 1990. Unpublished answers on "Core Heatup and Degradation (COR) Package" by R. M. Summers, Sandia National Laboratories, Second MELCOR Peer Review Meeting, November 5-7, 1990.

F-16 B. E. Boyack, Los Alamos National Laboratory, unpublished questions on "HS and COR Packages," by R. Visanta, in letter to B. E. Boyack (December 27, 1990). Unpublished answer on "Responses to Additional Viskanta Questions on HS Package Modeling" by R. M. Summers, Sandia National Laboratories, Third MELCOR Peer Review Meeting, January 11, 1991. Unpublished answer on "Responses to Additional Viskanta Questions on COR Package Modeling," by R. M. Summers, Sandia National Laboratories, Third MELCOR Peer Review Meeting, January 11, 1991.

F-17. R. M. Summers, "MELCOR Modeling Caveats," Sandia National Laboratories unnumbered draft (January 10, 1990).

F-18. W. T. Pratt, R. Denning, T. S. Kress, D. Powers, J. Gieseke, M. Khatib-Rahbar, and K. R. Perkins, "Evaluation of MELCOR as a Source Teim Analysis Code," Brookhaven National Laboratories unnumbered draft report (September 19, 1985).

F-19. J. W. Yang, "MAAP 3.0B Review/PWR Review, Part 2: In-Vessel Melt Progression," Safety and Risk Evaluation Division, Department of Nuclear Energy and Brookhaven National Laboratory draft document (August 1990). 
F-20. P. Hofmann, H. Uetsuka, A. N. Wilhelm, and E. A. Garcia, "Dissolution of Solid $\mathrm{UO}_{2}$ by Molten Zircaloy and its Modelling," International Symposium on Severe Accidents in Nuclear Power Plants, Sorrento, Italy (March 1988).

F-21. P. Hofmann and C. Politis, "The Kinetics of the Uranium Dioxide-Zircaloy Reactions at High Termperature","J. Nucl. Materials 87, pp. 375-397 (1975).

F-22. P. Hofmann, E. A. Garcia, and A. Denis, "Low-Temperature Liquefaction of LWR Core Components," Severe Accident Research Program Partners Review Meeting, Brookhaven National Laboratory (April-May 1990).

F-23 S. Hagen et al., "Interactions in Zircaloy/ $/ \mathrm{UO}_{2}$ Fuel Rod Bundles with Inconel Spacers at Temperatures Above $1200^{\circ} \mathrm{C}$," KfK report 4378 (September 1990).

F-24. P. Hofmann and M. Markiewicz, "Chemical Behavior of $(\mathrm{Ag} / \mathrm{In} / \mathrm{Cd})$ Absorber Rods in Severe Reactor Accidents," KfK report 4670 (August 1990).

F-25. P. D. Parsons, E. D. Hindle, and C. A. Mann, "The Deformation, Oxidation and Embrittlement of PWR Cladding in a Loss-of-Coolant Accident," Committee on the Safety of Nuclear Installation (CSNI) report ND-R-1351 (S) (September 1986).

F-26. P. D. Parsons and W. G. Burns, "Oxidation of Zircaloy Fuel Cladding and Hydrogen Evolution," in "PWR Degraded Core Analysis," J. H. Gittus, Ed., CSNI report ND-R610(S) (April 1982).

F-27. J. W. Yang and D. Mirkovic, "MAAP 3.0B Review/PWR Review, Part 1: RCS Thermal Hydraulics and Heat Transfer, Application for Success Criteria," Safety and Risk Evaluation Division, Department of Nuclear Energy and Brookhaven National Laboratory draft document (May 1990).

F-28. E. A. Boucheron, "TMI-2 Assessment Efforts," Sandia National Laboratory letter to R. B. Foulds, US Nuclear Regulatory Commission (September 21, 1989).

F-29. B. E. Boyack, "Final Minutes of the Third MELCOR Peer Review Meeting," Los Alamos National Laboratory Group N-12 letter N-12-91-171 (March 20, 1991).

F-30. F. Kreith, Principles of Heat Transfer, Third Edition, Intext Educational Publishers, New York (1973). 


\title{
APPENDIX G \\ Radionuclide (R/N) Package
}

by

\author{
J. A. Gieseke
}

\section{INTRODUCTION}

The radionuclide (RN) package deals with specifying the initial locations of fission products and their subsequent movement or redistribution throughout the core region, pressure vessel, coolant circuits, and containments. The models incorporated into the RN package analyze the behavior of gases, vapors, and airborne particulates as well as deposits on surfaces. Because fuel, structural materials, steam, and pools of water affect fission product transport, deposition, and retention, they must be included in the analyses.

Because of the large number of chemical species possible, a compression of these into chemically similar groups has been undertaken. The groups or chemical species have been variously defined to permit calculations for the specific kinetic situation of interest (e.g., release from fuel, transport, or in core-concrete interactions) and one function of the code is to map species among the various kinetic models. This has been necessary because the RN package was largely formed as an amalgamation of existing models. This process has the disadvantage of requiring sometimes rather complex mappings among models, but nevertheless has the advantage of relying on well-established and, in some cases, well-validated modeling efforts.

The various models are often handled independently but typically are interfaced so that they require an exchange of information among themselves. In particular, information is exchanged about geometries, flow rates, temperatures (e.g., fuel, water pool, surfaces, gases), pressures, material properties, mass-transfer coefficients, and amount of water condensed in a control volume. The RN package was defined as comprising the models (l) fission product location, mapping, and releases, (2) aerosol dynamics, (3) vapor condensation and evaporation, (4) decay heat power, and (5) fission product chemistry. These five major model areas were broken down further the MELCOR Primer (Ref. G-1) and that breakdown is maintained in this review.

\section{MODEL TITLE: INITIAL FISSION PRODUCT LOCATION}

\section{Model Description and Pedigree:}

This is basically a data input procedure for the user to give locations in the core for mass in each of the 15 fission product classes. It also accounts for later relocations of fuel mass among core cells as well as relocations from the core to the reactor cavity. In addition, fission products may be present initially in other locations or may be added from the DCH model later in the calculation.

The use of fission product groups by this model implies that the physical and chemical properties of one designated representative element adequately describes the properties of all elements in the group. It is further assumed that distribution of fission products within a control volume is uniform with the exception that distribution between fuel and fuel clad gap is specified within each core cell. 


\section{Implementation Within MELCOR:}

Although it is probably minor in impact, some care should be taken to see that appropriate sources and initial masses of nonradioactive components are obtained, probably from the ORIGEN code.

\section{Results of Model/Parameter Sensitivity and Benchmarking/Validation Studies:}

None found.

\section{Summary of Findings:}

The approach taken in this model seems reasonable. The concept of one representative element for each class seems to be a satisfactory and reasonable simplification. The use of an initial distribution of fission products, which is input by the user, is a practical approach.

The source term to the environment is dependent on an assumed initial inventory and hence on this model. The input available from existing ORIGEN calculations is often used in analyses but typically is not specific to many cases of interest. Nevertheless, the uncertainty in the input to this model is small compared with the uncertainty in other factors affecting release; therefore, the overall impact on uncertainties in the source term is small.

\section{Technical Adequacy:}

Category 1. 


\section{MODEL TITLE: FISSION PRODUCT MAPPING}

\section{Model Description and Pedigree:}

Mapping is provided by this model to distribute radioactive and nonradioactive materials vaporized within the core into 15 default classes. The distribution of masses from 15 classes into 29 VANESA species is also treated for materials transferring from the pressure vessel to the cavity, as well as the reverse process of mapping 29 species back into the 15 classes for transporting materials.

Although the bookkeeping seems complex, it is likely that little significant information is lost through the mappings upward and downward in numbers of classes. This negligible loss is a basic assumption of the process.

\section{Implementation Within MELCOR:}

Implementation takes a straightforward and common-sense approach.

\section{Results of Model/Parameter Sensitivity and Benchmarking/Validation Studies:}

None found.

\section{Summary of Findings:}

The mapping only redistributes materials among variously defined classes. The classes and distribution methods are reasonable. The mapping process is important in maintaining ease and speed of calculations.

\section{Technical Adequacy:}

Category 1. 


\section{MODEL TITLE: FISSION PRODUCT RELEASE}

Model Description and Pedigree:

In-Vessel. The release model for fission products from the fuel gives the release rate as a fraction of existing inventory per unit time as a function of temperature only. This rate can be modified to accommodate varying surface-to-volume ratios for the fuel. Either of two models can be used within MELCOR for the fuel: CORSOR or CORSOR-M. Each of these is an empirical fit to data that was available in about 1985 . The CORSOR representation is

$$
\text { release rate }(\mathrm{frac} / \mathrm{min})=\mathrm{A} \exp (\mathrm{BT}) \text {, }
$$

where $\mathrm{T}$ is the core cell temperature and the constants $\mathrm{A}$ and $\mathrm{B}$ are empirical class-dependent coefficients. A minimum threshold temperature for release is employed as is a maximum temperature above which the release rate is fixed. Coefficients and bounding temperatures are input by the user. The CORSOR-M representation is

$$
\text { release rate }(\mathrm{frac} / \mathrm{min})=\mathrm{k}_{0} \exp (-\mathrm{Q} / \mathrm{RT}) \text {, }
$$

where $\mathbf{k}_{0}$ and $Q$ are class-dependent coefficients.

Release rates predicted with either empirical equation are modified for predicting tellurium release by multiplying the predicted release rate by 0.025 if less than $70 \%$ of the zirconium in the core cell is oxidized. This calculational procedure results in the tellurium release rate being a severe siep function.

Fission products specified as being present in the fuel-cladding gap are released at the time of cladding failure given by a rupture temperature, candling, or oxidation. The COR package determines if the cladding has failed.

It is seen from the model that the fission product release from fuel is a function of bulk fuel temperature in each fuel node (not surface temperature) and on fuel surface area. The condition of fuel (burnup) is not assumed to be critical.

Cesium release is taken as the release of elemental Cs, which is converted to CsI as it is released, reacting irreversibly with all iodine released in the same time interval.

Core-Concrete Interaction. Release from the core-concrete interaction is treated by the VANESA code (Ref. G-2), which is a thermochemistry model for fuel melt interacting with concrete. The major release mechanism is by vapor transport carried out by bubbles passing through the melt. There is also some physical atomization by the bubbling process at the melt surface.

The oxygen potential and concomitant $\mathrm{H}_{2} / \mathrm{H}_{2} \mathrm{O}$ ratio are computed in the SRG subroutine in MELCOR by an iterative process. First the computer checks to see what metal(s) remain less than fully oxidized. If zirconium (Zircalloy) remains, then the initial estimate for $\mathrm{H}_{2} / \mathrm{H}_{2} \mathrm{O}$ is $10^{4}$. If all the zirconium is oxidized and chromium remains, then the initial estimate for the ratio is 10 . If chromium is fully oxidized, then iron sets the initial estimate as unity. After oxidation of the iron, nickel is considered and the $\mathrm{H}_{2} / \mathrm{H}_{2} \mathrm{O}$ ratio is initially set at 0.1 .

Because the $\mathrm{H}_{2} / \mathrm{H}_{2} \mathrm{O}$ ratio is a function of temperature, the Gibbs free energy of the oxide at temperature is utilized to come up with the final value by an iterative process, starting with the initial estimate

$$
\begin{gathered}
\mathrm{Zr}_{(\mathrm{c})}+\mathrm{H}_{2} \mathrm{O}_{(\mathrm{g})}=\mathrm{H}_{2(\mathrm{~g})}+\mathrm{ZrO}_{2(\mathrm{c})} \text { and } \\
\Delta_{\mathrm{fG}} \mathrm{G}^{\circ}=\mathrm{RT} \operatorname{lnK} \quad \mathrm{K}=\mathrm{H}_{2} / \mathrm{H}_{2} \mathrm{O}
\end{gathered}
$$


This approach appears sound, but inherent in the above equation is the condition that neither zirconium or zirconia are in solution with each other nor in solution in other substances.

VANESA performs its release calculations on a mole basis. The melt inventory is supplied to VANESA in kilograms for each chemical species. These masses are converted to moles in subroutine BCLTOV. Many of the radionuclides are combined into groups with similar chemical behavior to reduce the computational load. Because most components of these groups have significantly different atomic weights (i.e., $\mathrm{Rb}$ and $\mathrm{Cs}$ grouped as $\mathrm{Cs}$ or $\mathrm{Ce}, \mathrm{Np}$, and $\mathrm{Pu}$ grouped as $\mathrm{CeO}_{2}$ ), the conversion of moles to mass using the nominal atomic or molecular weight of the group yields masses that are incorrect. Although these errors are generally less than $10 \%$, it is a known source of error. The worst-case error normally occurs for the $\mathrm{CeO}_{2}$ group due to the large $\mathrm{Pu}$ inventory that is frequently present. The atomic weight error causes the released mass to be low.

The standard VANESA code calculates the concrete addition rate in the ith interval by a central-difference method for all but the first and last rate for intervals $\mathrm{i}-1$ and $\mathrm{i}$. Integration of the VANESA concrete addition rate does not yield the same silica amount specified in the input. The discrepancy arises because the average rate calculated from two intervals is only used during the second time interval. The integral of the concrete addition rate is equal to the input if forward differences are used for all intervals but the last. The error introduced in the calculations using central differences is minor, but it is known and easily corrected.

Additional minor problems appear to be present in the MELCOR version of YANESA. These involve the predictions of particles produced by bubble burst and calculations of thermodynamic properties of $\mathrm{CaO}$ and $\mathrm{CaOH}$. In addition, improvements are needed in the methods for approximating the free energies of the core melt species.

\section{Implementation Within MELCOR:}

Implementation for the in-vessel release model CORSOR/CORSOR-M appears satisfactory. The VANESA version used is a rather early version and would benefit from updating, and more importantly, from closer coupling to the CORCON model. The early VANESA version is known to have had numerous minor errors. It also appears there is no way to assign thermodynamic activities to the species or to utilize nonideal activity coefficients.

\section{Results of Model/Parameter Sensitivity and Benchmarking/Validation Studies:}

For high fractions released in vessel, the effect of coefficient changes is largely one of release timing and is probably significant. For materials with low fractions released, the effect of coefficient variations lead to changes in fractions released rather than just timing and the fraction released from the fuel is reflected proportionally in the fraction released from the plant.

Comparisons with SFD low burnup fuel studies indicate that CORSOR model predictions are not accurate for this case. For similar experiments with high burnup fuel, the agreement is reasonable indicating an important dependency on fuel burnup. The implemented models are appropriate for high burnup conditions.

In 1986, some verification test calculations were performed on VANESA (Ref. G-3) as well as the rest of the Source Term Code Package (Ref. G-4). The results indicated that the basic thermochemistry was correct but that releases were most strongly affected by the $\mathrm{H}_{2} / \mathrm{H}_{2} \mathrm{O}$ ratio. This pressure ratio comes from a different source in MELCOR. For one case where the release is not a function of the $\mathrm{H}_{2} / \mathrm{H}_{2} \mathrm{O}$ ratio, namely for $\mathrm{UO}_{2}$, the hand calculation showed $26.0 \mathrm{~g}$ of $\mathrm{UO}_{2}$ released as vapor, while VANESA showed $16.3 \mathrm{~g}$. This agreement seems satisfactory because VANESA considers kinetic factors that were ignored for the hand calculation. The $37 \%$ difference could easily be accounted for in this manner. Most species are strongly dependent on the oxygen potential for release and it is for this reason the $\mathrm{H}_{2} / \mathrm{H}_{2} \mathrm{O}$ ratio becomes so important. 


\section{Summary of Findings:}

The CORSOR models should be upgraded since time-dependent correlations and newer data are available for easy incorporation. A review of release rates for structural materials seems warranted as these release rates have been noted as being unreasonably high in some cases. Burnup for the fuel can have a significant effect on release rates and the analysis would benefit from its consideration.

The VANESA code calculations are unnecessarily conservative (overpredict release) through inability to assign thermodynamic activities and to utilize non-deal activity coefficients. A newer version of VANESA, preferably integrated into CORCON, should be used.

\section{Technical Adequacy:}

Category 5. 


\section{MODEL TITLE: AEROSOL DYNAMICS AGGLOMERATION}

\section{Model Description and Pedigree:} equation:

Aerosol agglomeration for a multi-component is described by the well-established

$$
\begin{aligned}
&{\frac{\partial q_{k}}{\partial t}(m, t)}=\int_{0}^{m} \phi(m-\mu, \mu) q_{k}(\mu, t) C(m-\mu, t) d \mu \\
&-q_{k}(m, t) \int_{0}^{\infty} \phi(m, \mu) C(\mu, t) d \mu \\
&-R(m) q_{k}(m, t)+S_{k}(m, t) \\
&+\delta_{1 k} \xi(m) C(m, t)-\frac{\partial}{\partial m}\left\{\xi(m) q_{k}(m, t)\right\}
\end{aligned}
$$

where

$$
\begin{array}{ll}
\mathrm{q}_{\mathrm{k}}(\mathrm{m}, \mathrm{t}) & =\text { mass distribution of } \mathrm{k}^{\prime} \text { th component, } \\
\emptyset(\mathrm{m}, \mu) & =\text { agglomeration kernel for particles of mass } \mathrm{m} \text { and } \mu, \\
\mathrm{C}(\mathrm{m}, \mathrm{t}) & =\text { particle number concentration of distribution, } \\
\mathrm{R}(\mathrm{m}) & =\text { removal rate for particles of mass } \mathrm{m}, \\
\xi_{(\mathrm{m})} & =\text { mass condensation rate onto particles of mass } \mathrm{m}, \\
S_{\mathrm{k}}(\mathrm{m}, \mathrm{t}) & =\text { mass source rate of } \mathrm{k}^{\prime} \text { th component, } \\
S_{\mathrm{k}}(\mathrm{m}, \mathrm{t}) & =\text { mass source rate of } \mathrm{k}^{\prime} \text { th component, and } \\
\delta_{1 \mathrm{k}} & =1 \mathrm{k}=1 \text { (water component) } \\
& 0 \mathrm{k} \neq 1 .
\end{array}
$$

This equation is transformed into a series of ordinary differential equations by using a mass discretization scheme that is then soluble using numerical integration schemes. The MELCOR model is based on the MAEROS model with the exception that water condensation is handled by an independent calculation. Agglomeration by Brownian, gravitational, and turbulent mechanisms are considered. kemel

Brownian agglomeration is computed by employing the standard expression for the

$$
\phi_{B}\left(r_{1}, r_{2}\right)=4 \pi k T\left[B\left(r_{1}\right)+B\left(r_{2}\right)\right]\left(r_{1}+r_{2}\right),
$$

where $\mathrm{B}(\mathrm{r})$ is the particle mobility function that includes an accounting for particle slip and where the agglomerating particles are of radii $r_{1}$ ano $r_{2}$.

Gravitational agglomeration is computed using the kernel

$$
\phi_{G}(r, R)=\pi(r+R)^{2}(U-u),
$$

where $u$ is the settling velocity of particle size $r$ and $U$ for $R$. Collision efficiency is corrected by a factor $E(r, R)$ and effective particle size for collisions is also adjusted. An additional factor reflecting possibilities for a sticking efficiency of less than unity is included even tholigh it appears as a product with $E$ and is not separable from agglomeration data. The MELCOR collision 
efficiency by Fuchs is used rather than the more widely accepted efficiency by Pruppacher and Klett. The difference is a factor of 3.

Turbulent agglomeration is given by two kernels that reflect particle motion with the fluid, $\phi_{s}$, and motion relative to the fluid $\phi_{1}$, which are functions of the turbulent energy dissipation rate, particle density and size, and the gas viscosity. The two kernels are combined as

$$
\phi_{\mathrm{T}}=\left(\phi_{\mathrm{S}}^{2}+\phi_{\mathrm{I}}^{2}\right)^{1 / 2} .
$$

The particle growth model is based on a number of assumptions and simplifications as follows:

(1) Particles are assumed spherical with correction factors making allowance for effects of nonspherical behavior on mobility and agglomeration.

(2) Particle size distribution is a continuum.

(3) All particles have the same material density.

(4) The aerosol size distribution can be bounded with minimal effect on the calculation of aerosol behavior.

(5) Agglomeration kernels are additive.

(6) Water condensation/evaporation can be treated independently from the aerosol governing equation.

(7) The control volume is well mixed.

(8) No electrostatic effects exist.

(9) The agglomeration kernel can be determined initially for a range of temperatures and pressures and then interpolated with sufficient accuracy.

\section{Implementation Within MELCOR:}

Implementation of the model within MELCOR seems satisfactory.

\section{Results of Model/Parameter Sensitivity and Benchmarking/Validation Studies:}

Sensitivity studies have demonstrated the importance of agglomerate shape factors when conditions are dry and open structures can exist.

Comparisons of MAEROS with contained aerosol behavior studies (dry conditions) show reasonable agreement. The same agreement can be assumed for MELCOR. Comparison with LACE (wet conditions) would be useful because of the importance of water condensation.

\section{Summary of Findings:}

The model is largely reasonable and seems properly implemented. The procedures for reducing the number of components to reduce computation time seem adequate and can be verified.

The use of an independent condensation/evaporation calculation for water vapor is questionable and should be verified. This should be possible by comparisons with other codes.

The truncation of the particle size distribution has caused problems with mass balance in some instances. In general, the default particle size range is for particles that are too large. The 
reported lack of calculational stability for the code when using smaller (reasonable) particle sizes is disquieting.

\section{Technical Adequacy:}

Category 1. 


\section{MODEL TITLE: AEROSOL DYNAMICS WATER CONDENSATION/ EVAPORATION}

\section{Model Description and Pedigree:}

This model is contained in the CVH package and the water calculated as being condensed is merely distributed over all particles with the relative amounts by size class being determined by the Mason equation. This calculation further assumes that there is no effect of surface tension (Kelvin effect) on vapor pressure over the droplets and no effect of dissolved lons on water vapor pressure.

\section{Implementation Within MELCOR:}

The calculation of condensation separately (in the $\mathrm{CVH}$ package) from the growth (agglomeration) equation is a questionable procedure.

\section{Results of Model/Parameter Sensitivity and Benchmarking/Validation Studies:}

None found.

\section{Summary of Findings:}

Separating condensation growth from other growth calculations is a questionable procedure. The validity of such an approach should be demonstrated by comparison with more exact models or by comparison with experiment. The Kelvin and solute effects are correctable deficiencies.

\section{Technical Adequacy:}

Category 5. 


\section{MODEL TITLE: AEROSOL DYNAMICS DEPOSITION}

\section{Model Description and Pedigree:}

Deposition of aerosols is treated within the governing aerosol behavior model and is covered by removal rates accounting for gravitational settling, diffusional deposition, thermophoresis, and Stephan flow (diffusiophoresis). Gravitational settling is calculated using a settling velocity given by the expression

$$
v_{s=\frac{2\left(\rho-\rho_{g}\right) g}{9} \mathrm{r}^{2} \mathrm{C}}^{9 x \eta}
$$

where $\rho$ and $\rho_{\mathrm{g}}$ are particle and gas densities, $\mathrm{g}$ is gravitational acceleration, $\mathrm{C}$ is the Cunningham slip correction factor, $\chi$ is the dynamic shape factor, and $\eta$ is the gas viscosity. velocity as

Diffusional deposition for particles onto all surfaces is given in terms of a diffusional

$$
v_{d}=\frac{k T C}{6 \pi \eta r \chi \Delta}
$$

where $k$ is Boltzmann's constant, $T$ is gas temperature, and $\Delta$ is the diffusional boundary layer thickness at the deposition surface (user input).

Particle motion down a temperature gradient (thermophoresis) given as the heat flux calculated in the (HSQFLX) package is divided by the thermal conductivity of the gas. The thermal deposition velocity is

$$
v_{1}=\frac{1.5 \eta \mathrm{C}}{\chi \rho_{\mathrm{g}} \mathrm{T}} \frac{\left(\mathrm{R}_{\mathrm{T}}+\mathrm{K}_{\mathrm{T}} l / \mathrm{r}\right) \nabla \mathrm{T}}{\left(1+3 \mathrm{~K}_{\mathrm{S}} l / \mathrm{r}\right)\left(1+2 \mathrm{~K}_{\mathrm{T}} l / \mathrm{r}+\mathrm{R}_{\mathrm{T}}\right)},
$$

where $l$ is the gas mean free path, $\mathrm{K}_{\mathrm{T}}$ and $\mathrm{K}_{\mathrm{S}}$ are thermal and slip coefficients, $\mathrm{R}_{\mathrm{T}}$ is the ratio of gas to particle thermal conductivity, and $\nabla \mathrm{T}$ is the temperature gradient.

Stephan Flow (diffusiophoresis) is the movement of particles to a surface along with a condensing vapor and is given for $\mathrm{W} \geq 0$ as:

$$
v_{d}=\frac{M_{s}^{1 / 2} W}{\left(m_{s}+m_{a}\right)\left(X_{s} M_{s}^{1 / 2}+X_{a} M_{a}^{1 / 2}\right)},
$$

and for $\mathrm{W}<\mathrm{O}$ as:

$$
\sqrt{d}=W / M 2
$$

where

$\mathrm{W} \quad=$ mass condensation rate,

$\mathrm{M}_{\mathrm{s}}, \mathrm{M}_{\mathrm{a}}=$ molecular weights of steam and air,

$X_{s}, X_{a}=$ mole fractions of steam and air, and

$\mathrm{m}_{\mathrm{s}}, \mathrm{m}_{\mathrm{a}}$ = mass concentration of saturated steam and air in the atmosphere. 
It should be noted that the above equation for $W>0$ differs from the form usually used but the numerical error is not significantly large (Ref, G-5).

In the use of these deposition mechanisms, the following assumptions are made.

(1) There are no electrical or turbulent effects on deposition.

(2) Deposition mechanisms are independent and the rates are additive.

(3) Stephan flow predominates and gas concentration effects are minimal in determining diffusiophoresis.

(4) High flows and complex geometries in RCS or reactor internals are assumed to be represented by open, well-mixed boxes in the same manner as the containment is treated.

\section{Implementation Within MELCOR:}

The implementation seems adequate although the deposition models are most suitable for analysis of containment volumes. The default value for the diffusional boundary layer seems too small. The use of an input value for $\Delta$, the diffusional boundary layer thickness, is a convenience for containment calculations where some estimates can be made to select a reasonable value. This is not the case when there is a directed flow such as in a primary coolant circuit where the situation determines diffusion to surfaces. Specific models are available for deposition from flowing gas streams onto surfaces. These models include the processes inertial deposition from turbulent flow, impaction from flow direction changes, diffusional deposition from turbulent flow, and impaction on obstacles (e.g., in reactor vessel, primary coolant circuit piping, steam separator, or steam dryer').

\section{Results of Model/Parameter Sensitivity and Benchmarking/Validation Studies:}

In general, deposition on walls is not often accurately predicted in comparison with experiments when using these deposition equations. Fortunately, sedimentation is usually predominant.

\section{Summary of Findings:}

The basic equations are reasonable representations of their specific mechanisms. Applications in various geometries (particularly RCS), for directed flow situations (RCS), and situations with combined inechanisms are not realistic because proper deposition models such as inertial deposition from flows, diffusional deposition from flows, and in special geometries (bends, steam separators, or dryers, etc.), for example, have been shown to be important and appropriate models are not included.

\section{Technical Adequacy:}

Category 4. 


\section{MODEL TITLE: AEROSOL DYNAMICS RESUSPENSION}

\section{Model Description and Pedigree:}

The model designates a fraction of deposits to be resuspended but no package in MELCOR computes or specifics this fraction.

\section{Implementation Within MELCOR:}

Not implemented.

Results of Model/Parameter Sensitivity and Benchmarking/Validation Studies:

None found.

Summary of Findings:

Resuspension is not believed to be of significance in affecting release or the source term so the lack of model implementation is satisfactory.

Technical Adequacy:

Category 7. 


\section{MODEL TITLE: AEROSOL DYNAMICS POOL SCRUBBING}

\section{Model Description and Pedigree:}

The models used are restatements of earlier models and include internal bubble circulation effects. However, bubble flattening is not treated directly but by inference from another theory. are included.

Particle deposition at a steam jet inlet is by vapor condensation only and no inertial effects

Deposition within bubbles is calculated for the mechanisms of Brownian diffusion, gravitational settling, inertial impaction, and evaporation/ condensation. However, no vapor pressure effect of dissolved ions on condensation growth of aerosols nor any growth of particles within a bubble is included.

A significant omission is that no vapor scrubbing in pools is considered.

\section{Implementation Within MELCOR:}

The treatment seems satisfactory except that corrections for flattened bubbles are applied from tabulated coefficients and this process seems unwieldy. There seem to be disagreements between the pool DFs computed with the MELCOR model and those calculations made using other codes. A general feeling is that there is likely an implementation error for the pool scrubbing model in MELCOR; however, any such error has not been located.

\section{Results of Model/Parameter Sensitivity and Benchmarking/Validation Studies:}

No comparisons with data were found.

\section{Summary of Findings:}

Aerosol collected in pools can be a determining factor on release in some instances. The treatment is largely adequate. Some improvement could be achieved by implementing the simple inlet impaction model from the Source Term Code Package and by adopting the vapor scrubbing model from the SPARC code.

\section{Technical Adequacy:}

Category 4. 


\section{MODEL TITLE: VAPOR CONDENSATION AND EVAPORATION}

\section{Model Description and Pedigree:}

The vapor condensation and evaporation models used in MELCOR were taken from the TRAP-MELT2 code (Ref. G-6). The equations have been well documented and extensively reviewed. The rate of change for the suspended concentration of any species is given by

$$
\frac{d C_{s}}{d t}=-\frac{A_{w} k_{w}}{V}\left(C_{s}-C_{w} s\right)-\frac{A_{p} k_{p}}{V}\left(C_{s}-C_{p}{ }^{s}\right)
$$

The rate of change of total condensed mass of vapor on walls is given as

$$
\frac{d M_{w}}{d t}=A_{w} k_{w}\left(C_{s}-C_{w}^{s}\right)
$$

and the rate of change for total mass of vapor condensed on aerosol particles as

$$
\frac{d M_{p}}{d t}=A_{p} k_{p}\left(C_{s} \cdot C_{p}^{s}\right)
$$

where

$\mathrm{C}_{\mathrm{S}} \quad=\mathrm{M}_{\mathrm{S}} \mathrm{V}=$ suspended vapor concentration,

$\mathrm{M}_{\mathrm{S}} \quad=$ total mass of the vapor suspended,

$\mathrm{M}_{\mathrm{W}} \quad=$ total mass of the vapor condensed on walls,

$\mathrm{M}_{\mathrm{p}} \quad$ = total mass of vapor condensed on aerosol particles,

$c_{p} s=$ equilibrium vapor concentration of the species of interest at the wall surface temperature,

$c_{W} s=$ equilibrium vapor concentration at the gas temperature,

$\mathrm{A}_{\mathrm{W}}=$ wall area

$\mathrm{A}_{\mathrm{p}} \quad=$ particle surface area, and

$\mathrm{k}_{\mathrm{w}}, \mathrm{k}_{\mathrm{p}}=$ mass-transfer coefficients from gas to wall or particle surface. assumptions:

Inherent in the model equations and their implementation in MELCOR are the following

(1) Particles are assumed to be at the gas temperature.

(2) Particle size (curvature) has no effect on vapor pressure at particle surface.

(3) Dilution of species in deposited layer does not affect partial pressure above surface (surface temperature only factor).

(4) Species in gas above surface is in equilibrium with same compound in surface layer.

(5) Particle motion through gas does not affect mass transfer $\left(k_{p}=D / r\right)$.

(6) Particles are assumed spherical and all of same composition.

(7) Mass-transfer coefficients are correctly imported from the HS package. 


\section{Implementation Within MELCOR:}

The implementation is inadequate for several reasons. First, some guidance should be provided on the limitations relating control volume size to the transport time for vapor diffusion across the volume. In other words, some guidance should be given on the applicability of a wellmixed volume model, or in a more practical sense, guidance given on the selection of control volume size. A second problem is that the numerical representation of the model is not correct for control volumes with multiple structures. The current method assigns condensed vapor mass to cool structures according to the structure number rather than assigning condensed mass according to available surface area and driving force.

\section{Results of Model/Parameter Sensitivity and Benchmarking/Validation Studies:}

Some indications of model sensitivities can be found from previous calculations for a variety of plants (Refs. G-7 and G-8). These calculations were performed with the same basic equations for fission product vapor condensation and evaporation. Comparison against a definitive set of experiments has not been found.

\section{Summary of Findings:}

The model for fission vapor condensation/evaporation is based on traditional, wellestablished equations. Questions of adequacy relate to the available data input for the calculations and the implementation in complex geometries. Some improvement is possible in accounting more accurately for vapor pressure of deposits through consideration of items such as vapor pressure reduction by dilution in the deposit and chemical interactions with surfaces. Checks on control volume size should be made to allow proper relationships between transport rates and condensation rates. The major problem seems to be incorrect numerics in implementation of the model.

\section{Technical Adequacy:}

Category 5. 


\section{MODEL TITLE: DECAY HEAT POWER}

\section{Model Description and Pedigree:}

The decay heat power model is divided into two parts, volume decay and surface decay, but the treatment for both is nearly identical. In either case the decay heat calculated for fission products either in the gas space or deposited on surfaces is apportioned by user input to (1) the gas space, (2) surfaces (other than the one with the heat source), and (3) the surface containing the heat source. In the case of the volume decay model, all surfaces are treated alike. The distribution is made as a fraction of all generated decay heat. In the case of liquid pools, all heat is retained in the pool.

\section{Implementation Within MELCOR:}

The model is not mechanistic and is implemented in a straightforward fashion.

\section{Results of Model/Parameter Sensitivity and Benchmarking/Validation Studies:}

None found.

\section{Summary of Findings:}

The model is zero order and nonmechanistic, although it allows for parametric variations of the decay heat distribution and hence it is possible to evaluate the sensitivity of accident progression and source term to variations in parameters.

\section{Technical Adequacy:}

Category 3. 


\section{MODEL TITLE: FISSION PRODUCT CHEMISTRY}

\section{Model Description and Pedigree:}

MELCOR allows simulation of selected chemistry effects involving reactions of fission product vapors or transfers among classes. The transfer rate of material from one vapor class to another class may be handled. This usually takes the form of vapor deposition onto a surface. The basic procedure is the same as for fission product condensation/evaporation but the overall transfer rates can be specified as a combination of a computed mass-transfer rate plus reaction rate or as an overall deposition velocity. Only forward or both forward and reverse reactions can be considered. In a similar fashion, transfers among vapor classes can be included.

\section{Implementation Within MELCOR:}

The chemistry simulation model appears to be correctly implemented as developed, although it is not mechanistically or physically correct. The practical aspects of choosing appropriate rates is not easily implemented.

\section{Results of Model/Parameter Sensitivity and Benchmarking/Validation Studies:}

None found.

\section{Summary of Findings:}

The fission product chemistry model relies on prior knowledge and is merely a method to simulate fast reactions. It does allow parametric investigations of potential chemical reactions and a practical method for handling reactions known for rapid transformations with known results. This parametric approach is only marginally adequate and should be replaced with a more physically realistic model, particularly for interactions of fission products on and with surfaces. Even though an improved model for treating surface chemistry is recommended, the overall fission product chemistry approach is deemed to be of marginal technical adequacy.

\section{Technical Adequacy:}

Category 4. 


\section{SUMMARY}

The RN package is a mixture of models ranging from highly sophisticated and accurate to very simple representations of physical or chemical processes. In some cases adequate models are missing and unavailable, and in some cases improved models are available and could be inserted as improvements. Among the potentially significant models noted as missing are those correlations that permit predictions of particle deposition in the reactor coolant system or on reactor vessel internals. Important models that are in need of improvement include those that treat water vapor condensation and evaporation, as these processes impact strongly on fission product behavior. Also at issue is the condensation/evaporation of the fission products themselves where questions exist regarding the reduction of vapor pressures at surfaces by dilution and chemisorption effects as well as the implementation of the basic equations within MELCOR. Models for fission product release from the fuel lack time dependence and are outdated.

The various models can be ranked for importance in terms of their need for remediation and impact. Prioritization of the models with the most important listed first is as follows:

(1) Aerosol Dynamics--Deposition Models

(2) Aerosol Dynamics-Water Condensation/Evaporation

(3) Vapor Condensation and Evaporation

(4) Fission Product Release

\section{REFERENCES}

G-1. R. M. Summers, "MELCOR Primer, Version 1.8.()," Sandia National Laboratory document (February 17, 1989).

G-2. R. M. Summers, R. K. Cole, Jr., E. A. Boucheron, M. K. Carmel, S. E. Dingman, and J. E. Kelly, "MELCOR 1.8.(): A Computer Code for Severe Nuclear Reactor Accident Source Term and Risk Assessment Analyses," Sandia National Laboratories SAND9()0364 (NUREG/CR-5531) (January 1991).

G-3. R. S. Denning, R. 0. Wooton, C. A. Alexander, L. A. Curtis, P. Cybulskis, J, A. Gieseke, H. Jordan, K. W. Lee, and S. L. Nicolosi, "Verification Test Calculations for the Source Term Code Package," Battelle Columbus Division report BMI-2140 (NUREG/CR-4656) (July 1986).

G-4. J. A. Gieseke, P. Cybulskis, H. Jordan, K. W. Lee, P. M. Schumacher, L. A. Curtis, R. 0. Wooton, S. F. Quayle, and V. Kogan, "Source 'Term Code Package-A User's Guide (Mod 1)," Battelle Columbus Division report BMI-2138 (NUREG/CR-4587) (July 1986).

G-5. S. A. Ramsdale, "A Review of the Aerosol Models in the MELCOR 1.6.() Code," United Kingdom Atomic Energy Authority, Safety and Reliability Directorate report (March 1988).

G-6. H. Jordan and M. R. Kuhlman, "TRAP-MELT2 User's Manual," Battelle Columbus Division report NUREG/CR-420.' (May 1985). 
G-7. J. A. Gieseke et al., "Radionuclide Release Under Specific LWR Accident Conditions," Battelle Columbus Division report BMI-21()4, Volumes I-VI (1984).

G-8. R. S. Denning, J. A. Gieseke, P. Cybulskis, K. W. Lee, H. Jordan, L. A. Curtis, R. F. Kelly, V. Kogan, and P. M. Schumacher, "Radionuclide Release Calculations for Selected Severe Accident Scenarios," Battelle Columbus Division report BMI-21.39, Volumes I-V (NUREG/CR4624) (1986). 


\title{
APPENDIX H \\ Core-Concrete Interactions (CAV) Package
}

\author{
by \\ Mohsen Khatib-Rahbar, Vijay K. Dhir, and Raymond Viskanta
}

\section{H.I. MODEL DESCRIPTION AND PEDIGREE}

\section{H.1.1. Overview}

In MELCOR, the interactions of core debris with the basemat concrete is modeled by the CAV package. This package integrates the CORCON/MOD2 code into the MELCOR architecture (Refs. $\mathrm{H}-1$ and $\mathrm{H}-2$ ).

CORCON/MOD2 models the thermal attack of core debris on concrete using a quasistatic multilayered approach consisting of the core debris pool, the concrete cavity, and the water and/or atmosphere above the debris pool. The decay and chemical reaction energy generated within the debris pool is transferred to the concrete basemat and sidewalls, as well as to the overlaying water pool and/or atmosphere.

Upon release on to the cavity basemat, the core debris is assumed to separate into layers of oxides and metals. Layer orders are determined by their relative densities (heaviest on the bottom). These layers consist of the dense oxide component rich in $\mathrm{UO}_{2}$ fuel, the metallic layer, and the light oxide layer that contains mostly concrete and steel oxides. Water is allowed to exist only on the top surface (the water is actually treated by the CVH package).

The partition of heat transfer is via corresponding thermal resistances (upward losses are sources to $\mathrm{CVH} / \mathrm{FL}$ and HS modules). Concrete ablation and decomposition generate gases and molten oxides. These decomposition gases are reduced as a result of interactions with the debris pocl metallic constituents to produce $\mathrm{H}_{2}$ and carbon monoxide gases. Both the reacted and unreacted decomposition gases eventually enter the atmosphere above the pool as sources to the $\mathrm{CVH} / \mathrm{FL}$ package.

\section{H.1.2. Conservation Equations}

The conservation of mass and energy equations are solved by the CAV (CORCON/MOD2) package, assuming an instantaneous spreading of the core debris to a preset maximum (the model neglects momentum conservation during the spreading process). The buildup of debris inside the cavity and/or pedestal region, following the reactor pressure vessel failure, is coupled to the COR package and modeled based on a time-dependent mass conservation principle.

\section{H.1.3. Energy Generation}

The energy generation within the debris pool is primarily due to decay heating, with contributions from chemical reaction energy. During a relatively short period, chemical reaction energy frorn $\mathrm{Zr}$ oxidation may be predicted to greatly exceed the decay heat contribution. In the MELCOR version of CORCON/MOD2, the decay heating is calculated by the Radionuclide (RN) and Decay Heat (DCH) packages. Heat sources based on Control Functions (CFs) and Tabular Functions (TFs) are also permitted (Ref. H-2). 


\section{H.1.4. Crust Formation and Freezing}

A quasi-steady one-dimensional analysis with the assumption that the heat generation rate equals the heat loss rate has been carried out. A crust is assumed to begin to form when the interface temperature drops below the freezing temperature. Crust thickness is determined by matching the energy generation rate with the net heat loss rate.

The implementation of the model within CORCON/MOD2 requires an iterative procedure, A possibility of obtaining nonphysical solutions exists. Breakup of crust upon interaction with water and instability of crust are not considered. The possibility of a formation of crust around the pool boundary has also not been included.

In the absence of water, these modeling uncertainties are not expected to have a significant impact on the overall model predictions.

\section{H.1.5. Debris Pool Layer Heat Transfer}

A pool is considered to be composed of several metal and oxide layers with well-defined interfaces. The CORCON/MOD2 model considers interlayer heat transfer as well as heat transfer at pool boundaries. Correlations reported in the literature for convective heat transfer with and without gas flow are used. Simple conduction solutions are obtained in case layers are stably stratified. Conventional correlation's for nucleate boiling, film boiling, maximum heat flux, and minimum heat flux are used. Radiation contribution in film boiling is also included.

Interlayer heat-transfer modeling is overly complicated in comparison to the models used elsewhere in the code. Correlations employed in the code do not necessarily represent the physical situation of interest. The effects of liquid subcooling and gas flow on Leidenfrost temperature and on boiling process have not been considered. The empirical constant used in the correlation for the maximum heat flux has been increased by about $20 \%$ (which is attributed to Rohsenow). Temperature dependence of physical properties is not modeled. Some inconsistencies exist with respect to modeling in the Heat Structure (HS) package and the CORCON/MOD2 (CAV) package. Thermal and hydrodynamic processes after vapor film collapse have not been adequately modeled.

\section{H.1.6. Melt Concrete Heat Transfer}

For a horizontal surface, a pseudo-film boiling model based on the concept of Taylor instability is used. For surfaces having an angle of inclination larger than $30^{\circ}$, a laminar or turbulent film model based on Reynolds analogy is used. An iterative procedure is required to solve for the heat-transfer coefficient on a surface with an angle of inclination larger than $30^{\circ}$. Surfaces with angle of inclination less than $15^{\circ}$ are assumed to behave like horizontal surfaces. Radiation heat transfer is also included in the model. The model will yield a lower heat loss rate from the pool for a given opacity of the environment. This discrepancy will become important at high temperatures.

At high-temperature differences, the effects of turbulence and interfacial waves on the gas film on a horizontal surface become important. The use of gas film flow path length in calculating heat and mass transfer is not supported by the laboratory data reported in the literature.

The model discrepancies will contribute to uncertainties in the rate of containment pressurization and concrete ablation.

\section{H.1.7. Debris Pool Surface Heat Transfer}

Energy transfer from the pool to the environment is considered by radiation and natural convection. For convection a constant heat-transfer coefficient of $10 \mathrm{~W} / \mathrm{m}^{2}-\mathrm{K}$ is assumed. The expression for radiation heat transfer assumes a finite opacity for the environment.

Thermal radiation is expected to be the dominant mode of heat transfer to the surroundings; therefore, radiative effects of aerosols must be included in the CAV model to 
account for changes in the atmospheric opacity. The standalone version of CORCON-MOD2. included a model for calculating atmospheric opacity based on an estimated aerosol concentration.

Uncertainties in the radiative properties will play a significant role at high temperatures. Evaporation at the pool surface and the effect of surface agitation on the convection heat-transfer coefficient have not been considered. Energy transferred by the liquid entrained by the gases has not been accounted for. An ad hoc approach is used to calculate the heat-transfer coefficient on surfaces having angle of inclination between $\mathrm{O}^{\circ}$ and $30^{\circ}$.

These models are approximate but are not totally unreasonable. The possibility of other gas flow configurations has not been considered.

\section{H.1.8. Concrete Decomposition and Ablation}

A quasi-static model is used to relate the heat flux at the surface to the concrete ablation rate. During decomposition of concrete, the mass generation rate of gas species is taken to be equal to the product of the partial density of species in the concrete and the ablation rate. Ablation energy includes contributions from both the sensible and chemical energies. Ablation temperature of concrete is assumed to lie between the liquidus and solidus temperature.

If debris temperature is below concrete ablation temperature, concrete surface is assumed to be adiabatic, thus neglecting a nonablative debris attack of concrete. Some discrepancies will also evolve when a major fraction of the pool is solid and when large fissures or cracks are present in the cavity.

\section{H.I.9. Chemical Reactions}

Chemical reactions of core debris constituents with sparging concrete decomposition gases are considered both within the debris pool and at the gas/film interface. A total of 38 species composed of 11 elements are included. Calculations are based on minimization of Gibbs function. Metals are oxidized to depletion in order of their reactivity. In the MELCOR version of CORCON/MOD2, an option is also provided to disable the so-called coking reaction (full reduction of $\mathrm{CO}_{2}$ by $\mathrm{Zr}$ to condensed $\mathrm{C}$ rather than $\mathrm{CO}$ ).

Reactions at the debris pool free surface have not been considered, and diffusion resistance on the gas side has not been included.

\section{H.1.10. Mass Transfer and Associated Heat Effects}

Change in enthalpy and mass of the material that drops into the debris pool from the top and passes through the pool as a result of ablation of concrete is made. Material is assumed to move slow enough so that it acquires the local temperature. Change in layer composition and enthalpy as a result of oxidation and as a result of material retention is considered.

The mass and energy transfer process is modeled on an ad hoc basis. No information is given as to the size and shape of the material that passes through the pool as a result of ablation of concrete. These deficiencies w.Il have little effect on final results. However, most of the ad hoc details could be avoided in favor of a well-mixed pool.

\section{H.1.11. Bubble Phenomena}

Void fraction is related to bubble rise velocity, which in turn is related to the terminal velocity. The entry size of bubbles is determined from Taylor instability considerations. Terminal bubble rise velocity is calculated for each layer. Correlations reported in the literature have been used.

Changes resulting from variations in pressure, temperature, and composition are accounted; however, coalescence or breakup of bubbles has not been considered. Chosen bubble size is not substantiated with experiments. Froth formation at the pool free surface and at the layer 
interfaces has not been considered.

Overall the model is adequate although there may be a substantial error in the calculated void fractions, which in turn will affect the convective heat-transfer coefficient.

\section{H.1.12. Material Properties}

The material propertles in the CAV package are those of the standalone CORCON/MOD2 code, and are independent of the water, the Noncondensable Gas (NCG), and the Materiul Properties (MP) packages in MELCOR. As a result, incompatibillties may exist in passing material, for example, between the COR and the CAV packages. Ad hoc adjustments to enthalpies are therefore necessary when materials are transferred into or out of the CAV package.

\section{H.2. IMPLEMENTATION WITHIN MELCOR}

Integration of the CORCON/MOD2 computer code into MELCOR is considerably improved when compared with the Source Term Code Package (STCP). Some of the most noteworthy implementation improvements include (1) a debris addition rate that is now time dependent and handled by the TP package from other MELCOR packages, (2) a coupling of CORCON to VANESA (part of the RN package) at every time step, and (3) consistent reduction of CORCON/MOD2 internal heating as a result of fission product releases from the melt/debris pool.

\section{H.3. RESULTS OF PARAMETER AND SENSITIVITY STUDIES}

MELCOR-specific parametric sensitivity studies for the CAV (CORCON/MOD2) package are nonexistent; however, extensive sensitivity calculations have been performed either with the standalone version, or the STCP version of the CORCON/MOD2 code.

Studies performed with the STCP version of CORCON/VANESA codes (Ref. H-3) revealed that, when coupled with VANESA, those parameters most significantly impacting fission product releases include the initial corium temperature and the chemical activity coefficients of VANESA. (In the MELCOR version of VANESA, the chemical activity coefficients are not available as sensitivity parameters!)

It should be noted that all of the existing sensitivity studies have been focused on the codc input parameters, while potentially large sensitivities to modeling assumptions have not yet been investigated.

\section{H.4. RESULTS OF BENCHMARKING/VALIDATION ACTIVITIES}

All of the benchmarking/validation studies performed to date are based on the standalone version of CORCON/MOD2, which are also relevant to the MELCOR-implemented version.

Numerous posttest comparisons have been made between the predictions of the standalone CORCON/MOD2 (and its evolutionary versions between MOD2 and MOD3) and results from Sandia National Laboratories and KfK/BETA experimental programs. Examples include the following (Ref, $\mathrm{H}-4$ and $\mathrm{H}-5$ ):

- CORCON/TURCIT (thermite): Good agreement to axial penetration measurements observed.

- CORCON/SWISS-1 (stainless steel melts, water poured on top): Good agreement with data observed.

- CORCON/SWISS-2 (stainless steel melt with water addition): Insproved heattransfer models provided better agreement with test data. Transient heat conduction intu concrete was found to be important in the early phase of the 
interactions.

- CORCON/TURC1SS (stainless steel melt): Code underestimated the measured penetration by a factor of 2-3.

- CORCON/BETA (high-power iron/alumina thermite): Code underestimated measured axial penetration.

- $\quad$ CORCON/TURC2(UO- $-\mathrm{ZrO}_{2}$ melts): Code overestimated the measured erosion by an order of magnitude.

- SURC.-1 \& $2\left(\mathrm{UO}_{2}-\mathrm{ZrO}_{2}-\mathrm{Zr}\right.$ mixture): Improved heat-transfer models provided better agreement with test data. Mixing models were identified to be needed for adequate treatment of condensed phase interactions. Need to improve the oxide phase diagrams was also identified.

Recent CORCON/MOD2 blind predictions of the Sandia SURC-4 (Sustained Urania Concrete) experiments revealed the following (Ref. H-6):

- Good agreement between CORCON/MOD2 and experimental data for prediction of downward penetration.

- CORCON underprediction of measured melt temperature, especially at the time of $\mathrm{Zr}$ addition.

- Qualitative agreement in total gas generation rates with CORCON prediction; however, impact of $\mathrm{Zr}$ on gas generation was not well predicted:

- underpredicted $\mathrm{CO}_{2}$ and $\mathrm{CO}$ generation rates

- reasonable hydrogen generation rate

- The CORCON/VANESA estimates of fission product releases were substantially lower than the SURC-4 experimental measurements.

Recent modeling changes incorporated into the CORCON-MOD3 have reduced the discrepancies in code prediction of SURC-4 data.

Even though the discrepancies in the CORCON prediction of experimental concrete erosion rates can be eliminated, there is insufficient data with real material to support the CORCON/MOD2 temperature predictions, as iission product releases (calculated by VANESA/RN package) are a strong function of CORCON/CAV-calculated melt temperature.

\section{H.5. IDENTIFIED DEFICIENCIES AND PLANNED IMPROVEMENTS}

MELCOR improvement needs have also been assessed and documented by Summers (Ref, H-7). These modeling improvements include

- models for mixing of oxidic and metallic debris,

- models for transient response of concrete,

- improved oxidic and metallic phase diagrams, and

- improved interface between CORCON and VANESA codes.

The modeling inadequacies identified as part of the SWISS and SURC experimental 
validation programs (Ref. H-6) for the standalone CORCON/VANESA codes are summarized as:

- coolant/melt heat-transfer models,

- axial heat-transfer models,

- $\quad$ oxidic and metallic phase diagrams, and

- assumptions of ideality (VANESA code).

These modeling improvements are apparently planned as part of the CORCON-MOD3 development effort as summarized by Bradley (Ref. H-6).

\section{H.6. SUMMARY FINDINGS}

\section{H.6.1. Review Findings and Relationship to Dominant Phenomena}

Table H-I provides a summary of phenomenological models in the CAV package, an assessment of model adequacy based on the guidance provided in Chapter 1, and an approach similar to that of Appendix D. In this approach, the phenomenological models of the CAV package are evaluated using the following key attributes:

Adequacy of Physics: This addresses the acceptability of the model physics as formulated in the CAV package.

State of Knowledge: This addresses the strengths of the available knowledge and/or data base (good, fair, or uncertain).

Adequacy of the Numerical Methods: This addresses the acceptability of the numerics as implemented in the CAV package.

Model Application: This addresses the applicability of the formulated models in the CAV package for use as predictive tools.

Model Implementation: This evaluates implementation of models for simulation of given process/phenomenon within the CAV package.

Importance of Phenomena: This addresses the importance of given process/phenomenon to prediction of severe accident progression.

\section{H.6.2. Summary and Conclusions}

Several deficiencies have been identified in various models included as part of the CAV package; however, the overall modeling approach appears adequate especially for dry cavity conditions.

Important modeling deficiencies have for the most part been recognized by the CORCON development group at Sandia, and most of the identified deficiencies are being addressed as part of the CORCON-MOD3 activities.

A prioritized summary list of Categories 4 and 5 as well as other important review findings is provided in Table $\mathrm{H}$-II.

The present models of the CORCON-MOD2 are deficient in several important areas as summarized below:

Heat Transfer to Concrete. The present gas film flow path length in calculating the heat-transfer coefficient on inclined surfaces is incorrect. The approach used to calculate heattransfer coefficients on surfaces having an angle of inclination between $0^{\circ}$ and $30^{\circ}$ is not mechanis- 


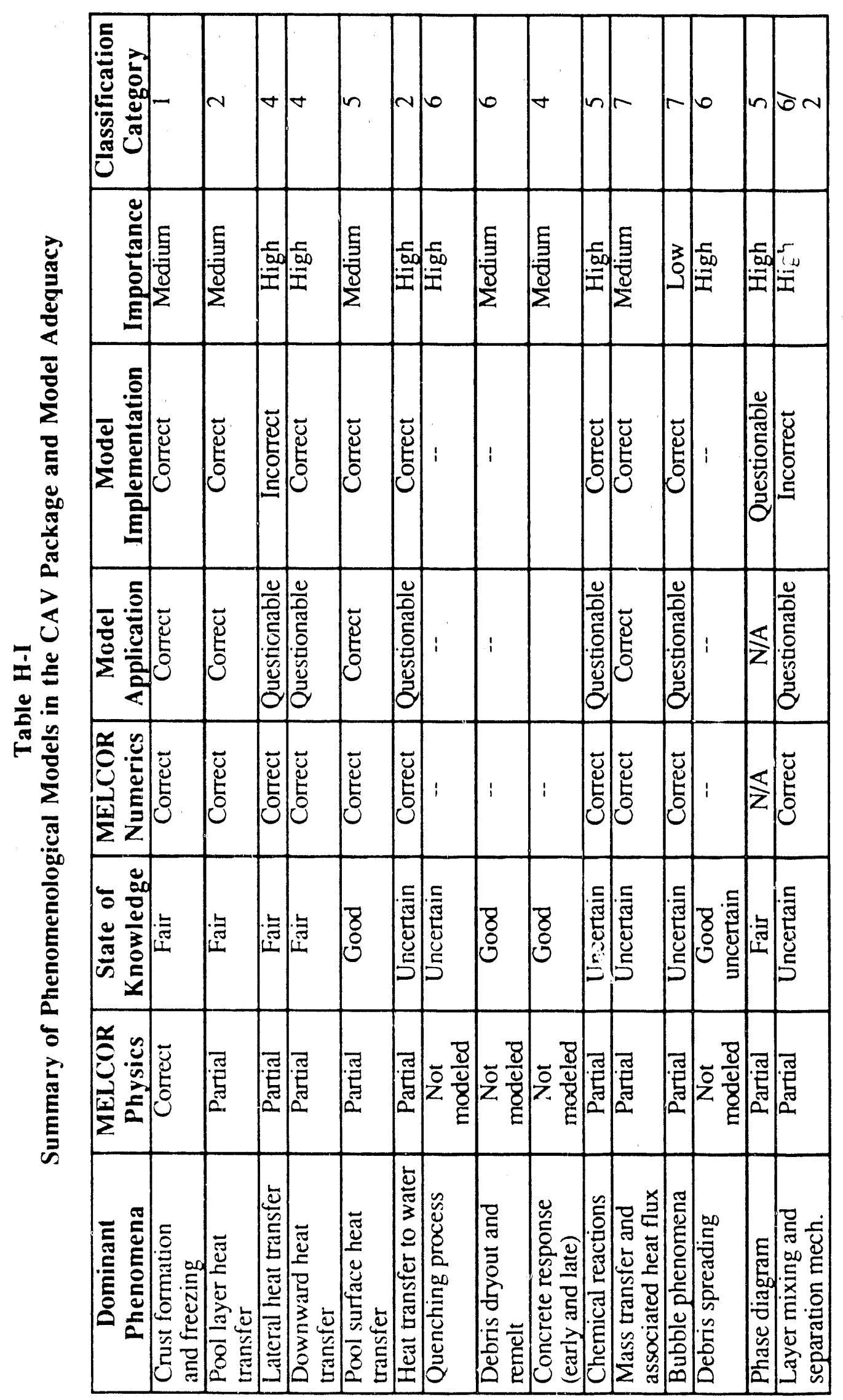


Table H-II

Prioritization of Review Findings for the CAV Package

\begin{tabular}{|l|}
\hline Heat transfer to concrete \\
. Lateral heat transfer \\
\hline Pool surface heat transfer \\
\hline Heat transfer to water \\
\hline Phase diagrams \\
\hline Concrete response \\
\hline Debris spreading \\
\hline Layer mixing and separation mechanisms \\
\hline CORCON/MOD2 and VANESA consolidation \\
(CORCON/MOD3) \\
\hline
\end{tabular}

tically justified. The ratio of downward to radial heat transfer to concrete has also been found to underpredict experimental data.

Pool Surface Heat Transfer. The radiative effects of aerosols must be included in the pool surface heat-transfer model to account for changes in the atmospheric opacity as a function of aerosol concentration.

Heat Transfer to Water. The modeling of thermal and hydrodynamic interactions with the overlying water pool is deficient. Models are needed to treat the potential for crust breakup, water penetration, fragmentation, quench, debris dryout, and remelt.

Debris Spreading. This is an important phenomenon, especially for BWRs with MARK-I and -II containments. The current CAV model assumes an instantaneous spreading of the core debris over the entire user-specified floor area. Debris thermal interactions with metallic structural surfaces are not included. Simple spreading models are available in the literature (e.g., Moody's model, MELTSPREAD model, etc.) and should be evaluated for implementation into the MELCOR/CAV package. Any spreading model should also include potential effects of flooding and direct contact interactions with metallic surfaces.

Oxidic and Metallic Phase Diagrams. Effects of eutectic formation on debris layer melting and solidification behavior are important. Improvements in CORCON/MOD2 phase diagrams are essential. Current incompatibilities in material properties between CAV and other MELCOR packages need to be eliminated.

Concrete Response. The present model neglects energy transfer to concrete if debris temperature is below concrete ablation temperature (nonablative attack). Transient conduction is important both during the very early and very late interaction phases.

Interlayer Mixing and Separation. The current layer separation is not based on a mechanistic quantitative criterion (layers are assumed to form). During the initial high-temperature phase of interaction, gas release from concrete is very vigorous, leading to intermixing of oxidic and metallic constituents. An interlayer mixing criteria along with a well-mixed debris pool model need to be developed.

CORCON/VANESA Consolidation. The current MELCOR integration of CORCON and VANESA is a considerable inprovement over the STCP; nevertheless, the consolidation of these modules into a unified code as currently planned for CORCON-MOD3 is desirable. This should also resolve the existing problem of duplicative but inconsistent chemistry between CORCON and VANESA codes. 


\section{REFERENCES}

H-1. R. K. Cole, Jr., D. P. Kelly, and M. A. Ellis, "CORCON-MOD2: A Computer Program for Analysis of Molten-Core Concrete Interactions," Sandia National Laboratories report SAND84-1246 (NUREG/CR-3920) (August 1984).

H-2. R. M. Summers et al., "MELCOR 1.8.0: A Computer Code for Nuclear Reactor Severe Accident Source Term and Risk Assessment Analyses," Sandia National Laboratories report SAND90-0364, R3 (NUREG/CR5531) (October 1990).

H-3. R. E. Davis et al., "QUASAR Screening Sensitivity Analysis: Application to the CORCON and VANESA Codes," Brookhaven National Laboratory technical report FIN A-3286, 6-22-87 (June 1987).

H-4. M. Silberberg et al., "Reassessment of the Technical Bases for Estimating Source Terms," US Nuclear Regulatory Commission report NURES-0956 (July 1986).

H-5. D. Bradley, "Status of CORCON-MOD3 Development and Validation," Presentation at Severe Accident Research Program Partner's Meeting, May 2, 1990.

H-6. M. Lee and R. A. Bari, "Comparison of Standard Problem SP-3 Calculations by Participants with the SURC-4 Experiment," Brookhaven National Laboratory technical report FIN A-3964, 5-27-88, Draft for Comment (June 1988).

H-7. R. M. Summers, "MELCOR Improvement Needs Assessment," Sandia National Laboratories unnumbered report (September 28, 1990). 


\section{APPENDIX I \\ Gas Combustion (BUR) Package}

by

M. A. Kenton

The MELCOR combustion model is very similar to the treatment in HECTR 1.5 except that the "continuous burn" option in HECTR is not included. Rriefly, the model calculates flammability, burn propagation to adjacent volumes, combustion completeness, and burn time using empirical formulas. Given the empirical basis of the model, it is felt that justification for its use must rely almost completely on how it compares to the available data. The validation efforts presented to the committee were quite limited. Nevertheless, it is judged that the model is adequate for most of its intended uses. However, it is recommended that additional flexibility be added to the model to allow parametric investigation of burns initiated by high temperatures. A similar recommendation was recently made for the CONTAIN code (Ref. I-1).

Each submodel is discussed separately below.

\section{MODEL TITLE: IGNITION CRITERIA}

\section{Model Description and Pedigree:}

The ignition limits are based on experiments and depend only on gas composition and whether igniters are present. LeChatelier's expression is used to relate $\mathrm{H}_{2}$ and $\mathrm{CO}$ concentrations.

Conditions under which detonations might be expected are flagged, but detonations themselves are not modeled.

\section{Implementation Within MELCOR:}

The user is allowed considerable latitude to change the constants in the default flammability correlations, but only within the context of the basic model. A control function cannot be used nor can ignition limits be set on a node-by-node basis.

\section{Results of Model and Parameter Sensitivity Studies:}

Grand Gulf calculations for a station blackout sequence were performed in A-12 to estimate containment loads from wetwell burns. The following werc varied:

- $\mathrm{H}_{2}$ concentration required for ignition: $5-12 \%$

- Steam concentration: dry and $55 \%$ by volume

The results of the calculations are shown in Table 2-3 Ref. 1-2.

Calculations of the response of the Browns Ferry reactor building to station blackout sequences investigated the impact of the concentration at which burns were initiated and showed a 
quite large impact on the results if "jet" burning took place as high-temperature containment gases flowed into the reactor building.

\section{Results of Model Benchmarking/Validation Activities:}

There do not appear to be any henchmarks of cases with igniters and continuous sources of hydrogen (e.g., the Accurex or Hydrogen Control Owners' Group-sponsored tests). There is a large set of data in the literature that is the basis for the original HECTR correlations for premixed situations, but it is important to keep in mind the distinction between flammability and ignition when no explicit ignition source is present.

\section{Identified Deficiencies and Planned Improvements:}

While flammability is fairly well understood, SNL considers ignition an area of high intrinsic uncertainty. Consideration is being given to removing all default correlations and other inputs.

\section{Summary of Findings:}

Because default correlations do not depend on temperature, it is up to the user to model the following phenomena by changing the defaults:

(1) diffusion flames at junctions caused by high-temperature jets entering a noninerted compartment (Refs. I-2--I-.5);

(2) recombination of $\mathrm{H}_{2}$ and $\mathrm{CO}$ with $\mathrm{O}_{2}$ in high-temperature regions (Ref. I-6, pages 3.4-36); and

(3) lean burns at igniters.

With regards to items (1) and (2), the user's job is made unnecessarily complicated by not being able to specify ignition limits using a control function or other means more general than currently allowed. It is believed that one must work around this limitation by lowering ignition limits everywhere and then using nodal combustion completeness correlations to prevent burns at low hydrogen concentrations where these are not desired. If igniters are not actually present, one could probably say there is an igniter only in certain compartments at certain times and ignition limits are lowered for igniters only. The user's guide and reference manual do not explicitly discuss modeling any of these effects, and these techniques are considered unwieldy at best.

\section{Importance Assessment:}

This model is very important. Over-conservatisms in the default treatment could strongly impact results, especially in Mark IIIs, ice condenser plants (Ref. I-7), and in BWR reactor building analyses (Ref. I-4) if the potential for relatively small burns to consume hydrogen and carbon monoxide is overlooked.

\section{Technical Adequacy:}

Default correlations are Category 2 except for burns induced by high temperatures. In view of the unnecessary difficulties that are imposed on a user attempting to model burns induced by high temperatures, Category 6 is assigned for these. 


\section{MODEL TITLE: BURN COMPLETENESS}

\section{Model Description and Pedigree:}

Default combustion completeness is based on experiment but is not a function of the concentration of the steam or other inertants.

\section{Implementation Within MELCOR:}

The user is allowed considerable latitude to change the constants in the default correlations. Alternatively, he can input a control function. The user can specify combustion completeness on a node-by-node basis.

\section{Results of Model and Parameter Sensitivity Studies:}

Grand Gulf calculations for a station blackout sequence were performed in Ref. I-2 to estimate containment loads from wetwell burns. The report states that the burn completeness was varied over the ranges observed in experiments at the Variable Geometry Experimental System (VGES), Fully Instrumental Test Site (FITS), and Nevada Test Site (NTS). However, in the cases with steam fractions of $55 \%$, the combustion completeness was assumed to be 10()$\%$ whenever the hydrogen fraction was $(0.08$ or greater. This conflicts with Ref. I-8, which indicates that combustion completeness was generally much lower at NTS, even for steam fractions of only $30 \%$, especially under quiescent conditions. This demonstrates the magnitude of the burden placed by a highly parametric model on the user.

Calculations of the response of the Browns Ferry reactor building to station blackout sequences investigated the impact of the concentration at which burns were initiated and showed a quite large impact on the results if "jet" burning took place as hot containment gases flowed into the reactor building.

\section{Results of Model Benchmarking/Validation Activities:}

Reference $1-8$ indicates that the default model can significantly overpredict final pressure for steam fractions of 3()$\%$ or greater under quiescent conditions. High steam fractions are quite common in PWR large, dry calculations and would also be expected in BWR Mark-III wetwells and in the upper containment regions of PWR ice condenser plants after loss of pressure. suppression. presented.

No benchmarks of cases with igniters and continuous sources of hydrogen were

\section{Identified Deficiencies and Planned Improvements:}

SNL considers this an area of high intrinsic uncertainty. Consideration is being given to removing all default correlations and inputs.

\section{Summary of Findings:}

It is up to the user to model diffusion flames at junctions caused by high-temperalure jets. This has been simulated by adjusting combustion completeness so that a complete burn occurs in the volume where the jet enters at a low combustible gas concentration. A better but still very simple model for jet burning could be written that would merely allow combustible gases 
exceeding a specified temperature 10 burn as they enter a non-inerted compartment; this modeling option was noted (somewhat disparagingly) in Ref. I-6, pages 3.4-53.

It is also up to the user to model diffusion flames or relatively small discrete burns at igniters. In the default model, igniters affect the ignition limit and, implicitly, the combustion completeness in a rather weak way. Specifically, the default ignition limit with igniters is ().(17, which implies a combustion completeness of about 7()\%. Thus, with a steady source of hydrogen, a series of relatively large burns would be predicted, though these would not ordinarily be of a magnitude sufficient to fail containment. This is quite different from much of the data with continuous sources of hydrogen, which shows that diffusion flames can develop in such a way that the hydrogen burns as fast as it enters.

The ability to model combustion completeness with control functions on a node-by-node basis could probably be used to model diffusion flames at igniters. No comments on such modeling were found in the documentation.

Finally, it is up to the user to model incomplete burning caused by high diluent concentration. It is believed that this too can, in principle, be done with control functions.

The user's guide and reference manual do not explicitly discuss modeling any of these effects. The model defaults will tend to predict larger than best-estimate burns if any of the following conditions apply:

(1) Very high temperature regions exist (these would lead to recombination if not inerted or jet burning if they were at relatively low average concentrations).

(2) Igniters are operational and their placement in containment is such as to promote diffusion fiames.

(3) Burns are calculated to occur at relatively high steam fractions.

\section{Importance Assessment:}

These models are very important. Over-conservatisms could strongly impact results, especially in Mark III, ice condenser plants (Ref. 1-3), and BWR reactor building analyses (Ref', I4).

\section{Technical Adequacy:}

Category 2 for ordinary deflagrations; Category 3 for igniters and jet burning. 


\section{MODEL TITLE: BURN TIME/COMBUSTION RATE}

\section{Model Description and Pedigree:}

Flame speed is calculated from either a default correlation, which depends on combustible gas and diluent concentration, a user constant, or a control function. The burn time is a user-input characteristic dimension of the volume (or the code can calculate this dimension based on a sphere of the same volume) divided by the flame speed evaluated at the beginning of the burn.

Combustion rate is adjusted continuously so that the final burn completeness is achieved at the end of the burn time.

\section{Implementation Within MELCOR:}

Flame speed and volume characteristic dimension may be entered, if desired, on a volume-by-volume basis.

\section{Results of Model and Parameter Sensitivity Studies:}

None known.

\section{Results of Model Benchmarking/Validation Activities:}

Presumably, the data on which the HECTR correlation was based is available but was not supplied to the committee.

\section{Identified Deficiencies and Planned Improvements:}

Default flame speed correlations are stated by the MELCOR documentation to be suspect if a high diluent concentration exists.

\section{Summary of Findings:} provided.

The model consists entirely of a correlation to experimental data. No validation was

\section{Importance Assessment:}

The model is believed to be important only if extremely short or long burn times apply or are incorrectly predicted; i.e., burn times are important if they are so long that the peak pressure is reduced by heat losses to passive heat sinks or to containment sprays, or if they are so short that there is insufficient time to vent the compartment.

\section{Technical Adequacy:}

Caltegory 2. 


\section{MODEL TITLE: BURN PROPAGATION}

\section{Model Description and Pedigree:}

Propagation of a burn from one volume into an adjacent volume is considered after " user-input fraction of the total burn time in the original volume has elapsed. LeChatelier's formula is used to check if the burn can progress into the recipient volume, considering whether the burn would need to propagate vertically, horizontally, or downward to spread in that volume.

\section{Implementation Within MELCOR:}

The user can change the constants in the default correlations, i.e., the combustible gas concentration required for a burn to propagate in the various directions can be changed. The user can also input the delay time reyuired for the burn to enter adjacent compartments.

\section{Results of Model and Parameter Sensitivity Studies:}

None known.

\section{Results of Model Benchmarking/Validation Activities:}

None known.

\section{Identified Deficiencies and Planned Improvements:}

Given the well-mixed control volume assumption in CVH, burned and unburned volumes are not separately tracked. Therefore, as the burn progresses, burned and unburned gases will be displaced in proportion to their compartment average mass fractions (so called "homogeneous burn" approximation). SNL states that the HECTR staff consider this a serious problem that is being worked on for the standalone version of the code.

\section{Summary of Findings:}

The model appears reasonable for integrated severe accident analyses.

\section{Importance Assessment:}

The model is somewhat important.

\section{Technical Adequacy:}

Category 2, based on homogeneous burn approximation. 


\section{REFERENCES}

1-1. N. K. Tutu et al, "Estimation of Containment Pressure Loading Due to Direct Containment Heating for the Zion Plant," Brookhaven National Laboratory report NUREG/CR-5282 (Murch 1991).

1-2. S, E. Dingman et al., "MELCOR Analyses for Accident Progression Issues," Sundia National Laboratories unpublished draft report SAND89-()()72 (NUREG/CR-5331).

1-3. R, W. Wierman, "Experimental Study of Hydrogen Jet Ignition and Jet Extinguishment," HEDL-TME-78-8() (April 1979).

I-4. S. R. Greene, "The Impact of BWR MK I Primary Contaimment Failure Dynamics on Secondary Containment," presented at The 15th Water Reactor Safety Research Information Meeting, Gaithersburg, Maryland (NUREG/CP.()()9()) (October 29, 1987).

1-5. S. E. Dingman and J. E. Kelly, "MELCOR Analyses of Drywell Flammability," Transactions of the American Nuclear Society 60 (1989).

1-6. G. G. Weigand, "Thermal-Hydraulic Process Modeling in Risk Analysis: An Assessment of the Relevant Systems, Structures, and Phenomena," Sandia National Laboratories report SAND84-1219 (NUREG/CR-3986) (August 1984).

1-7. C. Wong, "Influence of Natural Convection and Diluent Inerting on $\mathrm{H}_{2}$ and $\mathrm{CO}$ Oxidation in the Reactor Cavity," Naclear Engineering and Design 107, 271-282 (1988).

1-8. C. C. Wong, "HECTR Analyses of the Nevada Test Site Premixed Combustion Experiments," Sandia National Laboratories report SAND87-()956 (NUREG/CR-4916) (November 1988). 


\section{APPENDIX J \\ Engineered Safety Features (ESF) Package}

by

M. A. Kenton

The ESF package contains two independent models for PWR fan coolers and containment sprays. It is convenient to address each separately.

The fan cooler model is based on a very simple correlation to experimental data that was formulated for the MARCH code. This formulation is not physically based. Other codes used for severe accident analyses, for example, CONTAIN and MAAP, employ mechanistic models that are considerably more detailed, and can be expected to be much more accurate when applied to conditions far more severe than was represented by the test data. This renders the MELCOR fan cooler model unsuitable for AM work. However, for PRA applications it is judged that the anticipated errors are not of great concern, and the model is thus assigned to Category 7.

The containment spray model is based on the model in HECTR and is thought to be similar in treatment and level of detail to that used in other severe accident codes. The model does not consider radiation and is limited to use in atmospheres that contnin a significant noncondensable concentration. Nevertheless, the spray model is considered adecuate for its intended purposes and is assigned to Category 1.

\section{MODEL TITLE: FAN COOLER}

\section{Model Description and Pedigree:}

The model is intended to calculate total heal removal and steam condensation rate from the containment atmosphere as a result of the PWR fan cooler operation and has also been used to represent BWR reactor building room coolers (Ref, J-1). The model is a modified version of the model in the MARCH code, but very little justification for its adeguacy is available.

A total heat-transfer coefficient was derived from performance data on a fan cooler given in a previous revision of the Oconee FSAR. The low steam mole fraction limit of this heat-iransfer coefficient is assumed to represent sensible heat transfer and is denoted as $h_{h}$. This is assumed to be constant regardless of containment conditions. The remainder of the total heat-transfer coefficient (linear in steam mole fraction) is assumed to represent condensation and is denoted as $h_{m}$.

The "effective area" $A_{\text {clr }}$ is defined so that the rated heat removal is obtained from the fan cooler under design conditions.

The sensible heal trantifer is then given by:

$$
Q_{h}=h_{h_{1}} \Lambda_{\text {elf }}\left(\mathrm{I}_{\mathrm{p}}-\mathrm{T}_{\mathrm{s}}\right) \text {, }
$$

where $\bar{T}_{p}$ is the average temperature of the gas palssing through the unit and $\mathrm{T}_{\mathrm{s}}$ is the average cooling water temperatture. The heat removal due to condensiltion is given by:

$$
Q_{m}=h_{m} A_{\text {eff }}\left(T_{p}-T_{s}\right) .
$$


Limits are placed on $\mathrm{Q}_{\mathrm{m}}$ to prevent a negative exit steam flow rate.

\section{Implementation Within MELCOR:}

The fan cooler condensate is discharged into the node in which the fan cooler takes suction; this is not always the case in actual containments.

Sensitivity coefficients allow the heat-transfer correlations to be altered.

\section{Results of Model and Parameter Sensitivity Studies:}

Only one study is known to exist. In calculations for LaSalle performed as part of Ref, J-1, room cooler rated heat removal was varied. In this case, the room coolers were overwhelmed by the flow of hot gas from the containment, and the variation in rated heat removil capability made little difference in the results.

\section{Results of Model Benchmarking/Validation Studies:}

The only known validation study (Ref. J-2) compared the MARCH model (which is similar but not identical to the MELCOR model) with a detailed CONTAIN calculation. Input parameters were chosen in such a way that the two models were matched at saturated conditions. When gas temperature was then increased by $20 \mathrm{~K}$, a $10 \%$ overprediction in heat removal was calculated by the MARCH model. When the air content was doubled, the MARCH model underpredicted heat removal by $7 \%$. These results suggest that the model will be inaccurate when applied to adverse containment conditions.

\section{Identified Deficiencies and Planned Improvements:}

No improvements are planned.

\section{Summary of Findings:}

The functional forms of the heat-transfer coefficients are assumed to be the same for all PWR and BWR coolers under all accident conditions. That is, the sensible heat-transfer coefficient is constant, and the heat-transfer coefficient representing condensation varies linearly in the steam mole fraction. Neither assumption is correct.

The driving force for condensation is temperature rather than steam density or partial pressure difference. The model thus does not represent the underlying physics.

The fan cooler is represented as only one volume, i.e., the change in conditions of the inlet gas and the cooling water as they pass through the unit is represented only by using average temperatures in the heat-transfer correlations. The averaging technique is crude and the outlet gas flow can be undersaturated based on the local water temperature.

Note that the Oconee FSAR no longer has the data on which the model is based. It appears that the utility concluded that a subsequently developed mechanistic model had to be used for reliable results.

No basis exists to apply the model to conditions that might be seen in severe accidents. One would expect the model to overcalculate steam condensation at high superheats. It is possible that the model is not even accurate under DBA conditions. 


\section{Importance Assessment:}

For most PRA applications, it is judged that errors in fan cooler modeling are relatively unimportant, because either the fan coolers have far more capacity than is needed to remove decay heat or because the fan coolers are assumed inoperative.

For recovery scenarios investgated as part of AM work, expected errors in condensation rate would impact assessments of the dangers of de-inerting the containment atmosphere and causing burns.

There would also be a concern if relatively low-capacity units (room coolers and nonsafety grade fan coolers used for normal heat loads) were thought to be important and were modeled, e.g., for environmental qualification assessments.

\section{Technical Adequacy:}

Category 7 for PRA and Category 4 for AM calculations. 


\section{MODEL TITLE: SPRAY MODEL}

\section{Model Description and Pedigree:}

The spray model calculates heat and mass transfer by tracking a representative water droplet from its point of origin until it contacts the floor. The model is virtually identical to that used in HECTR.

Droplets are assumed to be spherical, isothermal, small enough to ignore internal resistance, and fall at their terminal velocity. Atmospheric conditions are assumed not to change during the droplet fall time (quasi-steady treatment).

Multiple drops sizes are allowed. However, if the RN package is in use, only one drop size may be used, and only one given spray flow may be modeled in any given control volume. neglected.

Drops may leave one control volume and enter another. Radiation to droplets is

\section{Implementation Within MELCOR:}

The user must write control functions if he/she wishes to represent the delivered flow as a function of containment pressure and spray pump alignment.

The user must also construct a control function to calculate the initial droplet temperature in PWR recirculation mode or for BWR sprays when their source is the suppression pool.

Results of Model and Parameter Sensitivity Studies:

None known.

\section{Results of Model Benchmarking/Validation Activities:}

The SNL roadmap document for ESF (Ref, J-3) states that CSE test A-9 was simulated with MELCOR but was not documented.

\section{Identified Deficiencies and Planned Improvements:}

No improvements are planned.

\section{Summary of Findings:}

Standard textbook correlations are used to represent heat and droplet. The model appears adequate for most of the intended uses, although it cannot be used in very high steam fraction environments, e.g., in the drywell under some circumstances or in the RPV, pressurizer, or steam generator.

\section{Importance Assessment:}

For most PRA-related purposes, containment sprays have such a high heat removal rate that relatively large errors can be tolerated. The inability to model primary and secondary system sprays will impact the ability to perform success criteria evaluations (if this is ever done) and to otherwise simulate complicated transients. 


\section{Technical Adequacy:}

Category 1 (for containment use). No model is available in MELCOR for primary system applications or for use in a nearly pure steam containment environment (e.g., a completely purged drywell or a PWR containment after containment failure).

\section{REFERF,NCES}

J-1. S. E. Dingman et al., "MELCOR Analyses for Accident Progression Issues," Sandia National Laboratories unpublished draft report SAND89-0072 (NUREG/CR-5331).

J-2. G. G. Weigand, "Thermal-Hydraulic Process Modeling in Risk Analysis: An Assessment of the Relevant Systems, Structures, and Phenomena," Sandia National Laboratories report SAND84-1219 (NUREG/CR-3986 (August 1984).

J-3 R. M. Surnmers, "Transmittal of MELCOR Roadmaps," Sandia National Laboratories letter to B. E. Boyack, Los Alamos National Laboratory, containing list of roadmaps (August 14, 1990). 


\title{
APPENDIX K
}

\author{
Properties Packages
}

by

T. J. Haste

\section{K.1. INTRODUCTION}

This section summarizes the main points from the detailed reviews of the models in the properties packages, which comprise the Control Volume Thermodynamics (CVT) package (which itself makes use of the noncondensable gas (NCG) and water properties (H2O) packages to obtain basic data), the Physical Properties (MP) package, and the Decay Heat (DCH) package. The Transfer Process (TP) package, which controls the transfer of core material between pairs of packages but which itself contains no physical modeling, is also briefly discussed.

\section{K.2. CONTROL VOLUME THERMODYNAMICS}

Calculation of the thermal behavior of water and noncondensable gases has a central role in modeling the hydrodynamic behavior of core and containment in all stages of a postulated reactor accident. The review of the properties package includes an overview of the CVT package, which provides thermodynamic data; however, assessment of the basic MELCOR approach of dividing each thermal-hydraulic control volume into a pool and atmosphere is taken into account in the section devoted to the Controi Volume Hydrodynamics (CVH) package, with which the CVT package is closely linked. It is noted, however, that there is no true disequilibrium model (which would support, for example, the presence of superheated steam and subcooled water droplets). This imposes limitations on mechanistic AM analysis involving reflooding scenarios.

The modeling in the $\mathrm{H} 2 \mathrm{O}$ package follows suund principles and is judged entirely adequate. The databases used are well established. The analytic formulation avoids discontinuities and inconsistencies in the supplied data, though this treatment does result in run-time penalties. In the NCG package, the origin of some of the preset basic data is unclear, but the principles of the modeling are again judged sound and any contribution from these packages to uncertainty in the calculation of the source term is most unlikely to be significant. The assessment of this part of the code is summarized in Table K-I.

\section{K.3. MATERIAL PROPERTIES}

The material property package provides a wide range of thermophysical data for reactor materials as well as other properties such as thermal conductivity and binary diffusivities for fluids. User-defined materials may be specified, but the property set for each such material is overdefined with no check on the internal consistency, a lack of which can lead to run-time errors.

There appear to be no serious errors in the thermal properties of the major materials; core heatup data from experiments such as PBF SFD 1.1 and 1.4 (Refs. K-1 and K-2) along with Phebus B9+ (Ref. K-3) show good agreement with MELCOR calculations. However, there is a need for a some tidying-up of the documentation and clear identification of the sources of the data, while improving the calculation of thermal conductivities and viscosities of gas mixtures. Some work on the latter has been reported (Ref. K-4). More sensitivity coefficients would be useful, 
e.g., for binary diffusivities. If eutectic reactions are included in the COR package, properties for eutectic mixtures will be needed from MP; data in this area are sparse.

The broadly adequate nature of this package is reflected in the detailed assessments and in the summary provided in Table K-II. The package is unlikely to give rise to significant errors in the source term calculation.

\section{K.4. DECAY HEAT}

The DCH package provides a considerable amount of flexibility for the user to choose among a number of physically reasonable, state-of-the-art options with the facility to carry out sensitivity studies. No serious deficiencies have been identified and the package is judged wholly adequate. Summary findings are given in Table K-III.

\section{K.5. TRANSFER PROCESS PACKAGE}

The package deals with the transfer problem in a reasonable way, but leaves the details of the transfer matrices to the user (though defaults are supplied). The code developers plan in future to make the transfer processes more transparent to the user.

\section{K.6. CATEGORY 4 OR 5 FINDINGS}

There are no independent Category 4 or 5 items in the MELCOR properties package. The need for material properties for eutectics is intimately connected with the need to model eutectic phenomena themselves (in the COR package) and is therefore not highlighted as a separate item here.

\section{K.7. GENERAL IMPRESSION}

The overall impression is that the MELCOR code is generally satisfactory in the areas of material property and decay heat modeling. The main need is to tidy-up the MP package. Uncertainties in source term calculations arising from any of these packages are judged to probably be small. 


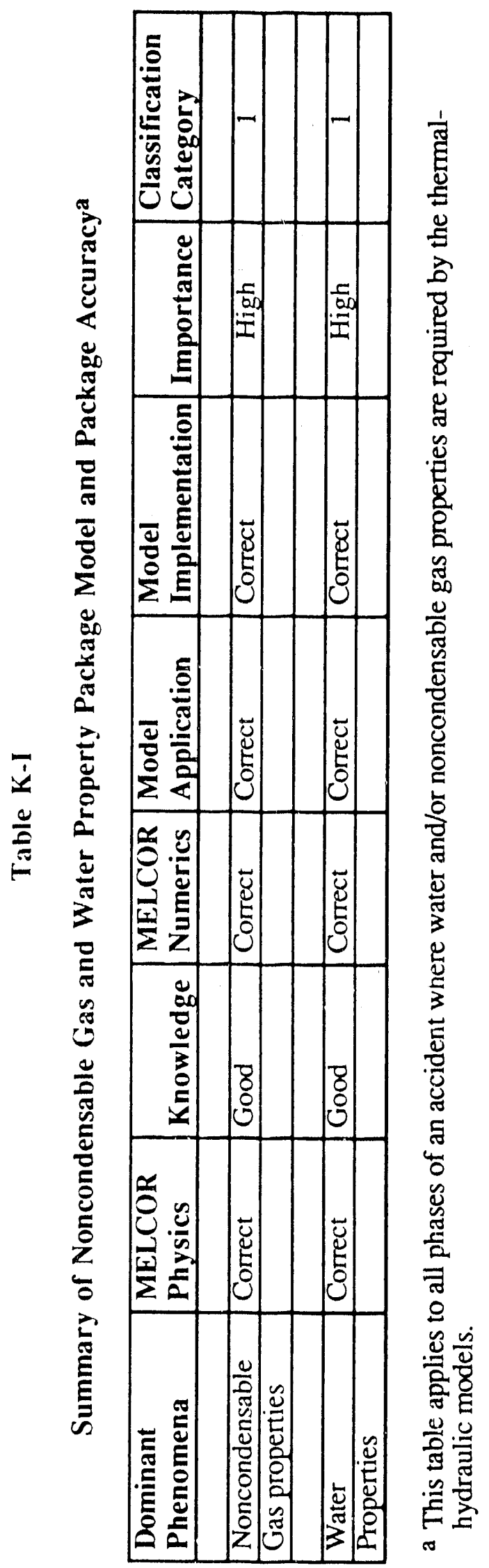

K-3 


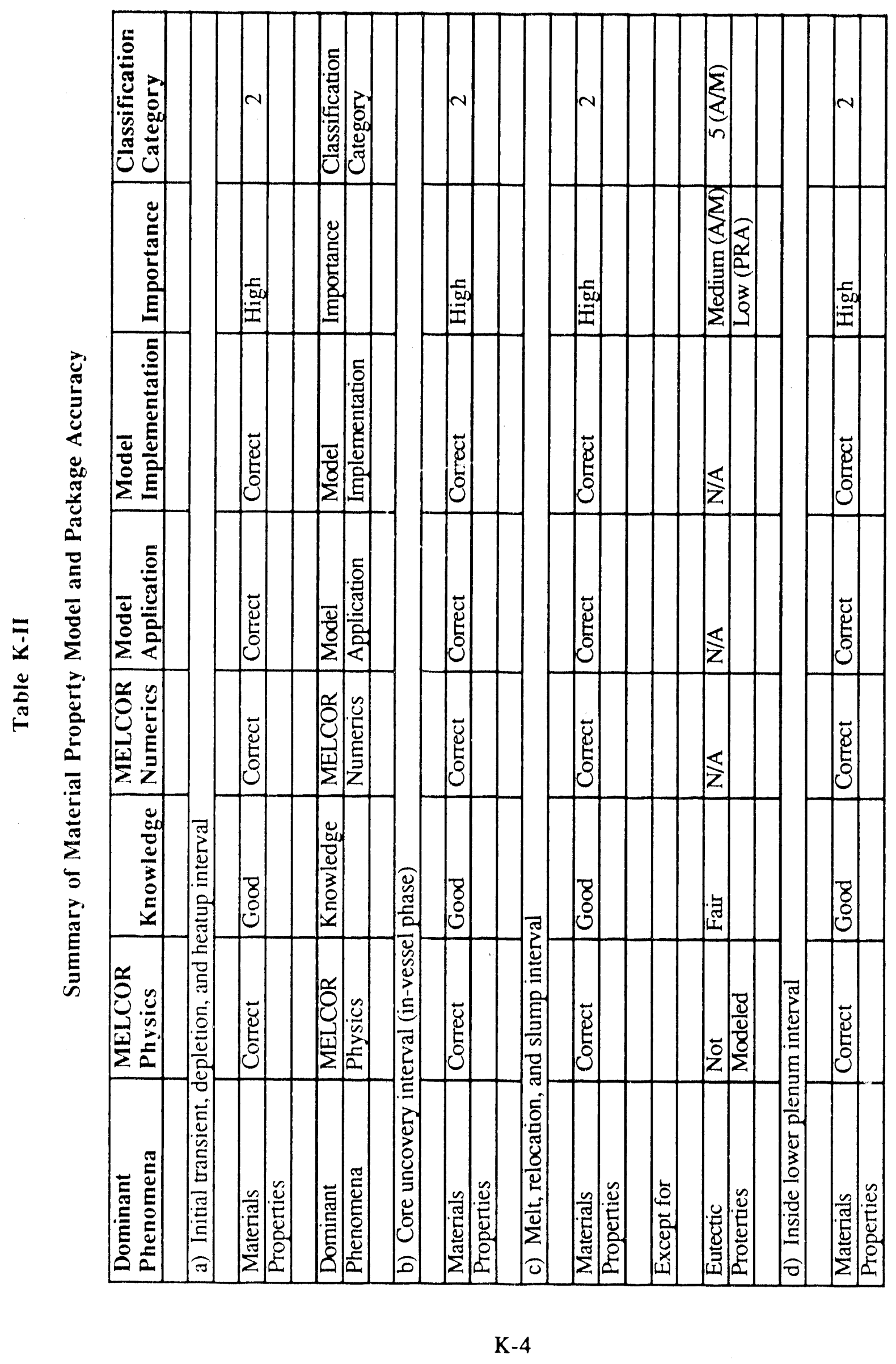




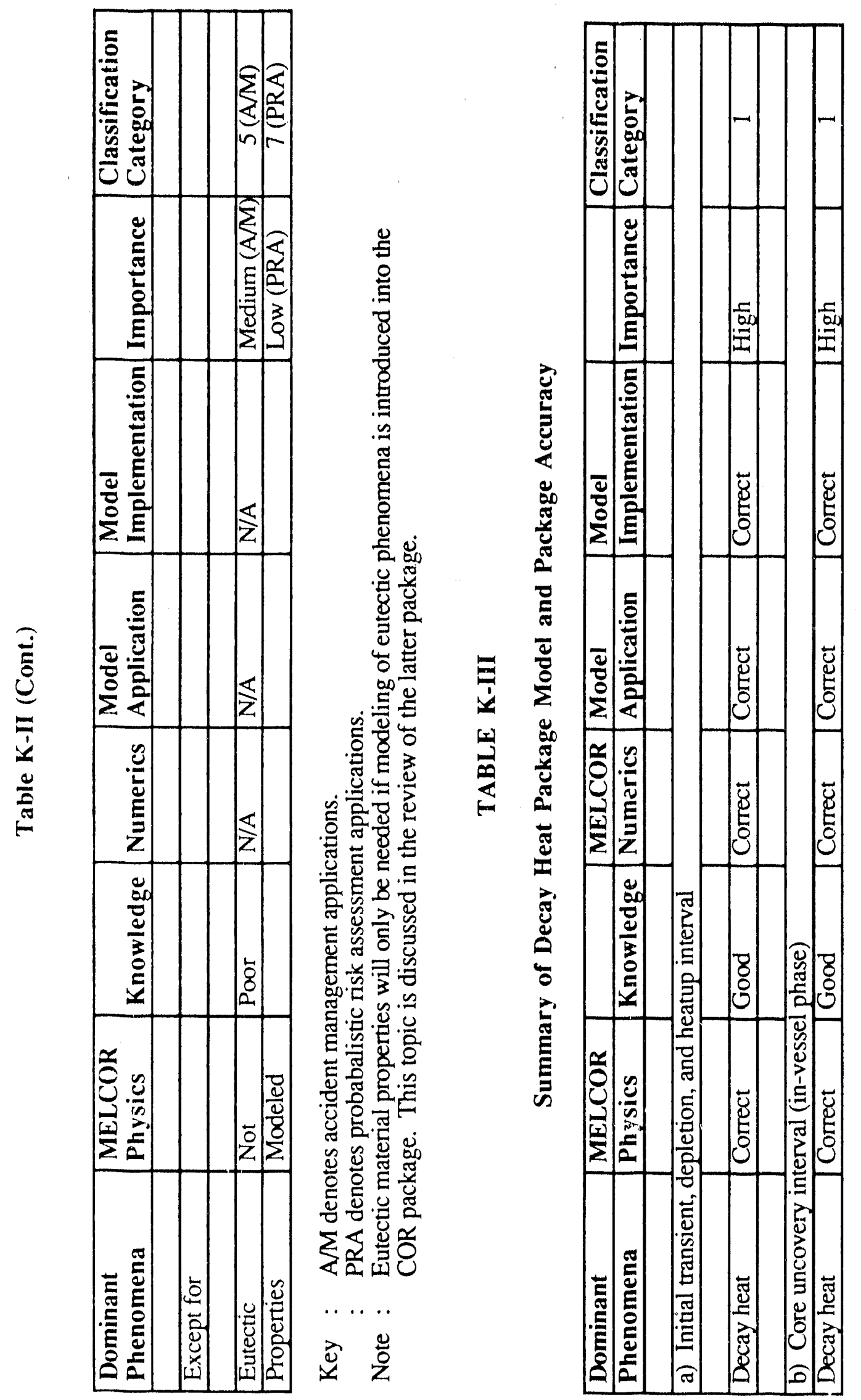




\section{MODEL TITLE: CONTROL VOLUME THERMODYNAMICS}

\section{MELCOR Package Identifier: CVT}

\section{Package Modeling Concepts:}

The package calculates the thermodynamic state of the materials in each volume using the volume, energy, and masses from the CVH package.

Each volume is divided into an atmosphere and a pool. In the "equilibrium" option these are in thermal and mechanical equilibrium. In the "nonequilibrium" option, only mechanical equilibrium exists (each part is in equilibrium internally, not a true disequilibrium model). These concepts, along with the partitioning of the water phases between the pool and the atmosphere, are dealt with implicitly in the review of the CVH package.

\section{Relationship to Dominant Phenomena:}

The package is relevant to the initial transient, heatup, core uncovery, discharge, and blowdown (all hydrodynamics and transport).

\section{Package Governing Equations:}

The governing equations are fully described in the Ref. K-5. The H2O and NCG packages are called to provide basic thermodynamic data for water/steam and non-condensable gases, respectively; these packages are reviewed separately.

\section{Completeness of Modeling Package:}

In view of the close connection between this package and the CVH package, the two packages were assessed together by using thermal-hydraulic "baby problems." The completeness of the present package is therefore discussed in the relevant section on CVH.

\section{Package Numerics:}

The package uses an analytic formulation, which ensures good consistency. However, the procedure is computationally expensive, which probably accounts for the slow-running characteristics of $\mathrm{CVH}$. 


\section{MODEL TITLE: DECAY HEAT POWER}

\section{MELCOR Package Identifier: DCH}

\section{Package Modeling Concepts:}

The package provides decay heat powers as required by other packages. It does not treat fission product transport or chemical interactions; these are dealt with elsewhere, e.g., in the Radionuclide (RN) package.

\section{Relationship to Dominant Phenomena:}

Decay heat is itself a dominant phenornenon.

\section{Package Governing Equations:} options.

There is a choice among an ORIGEN-based elemental/group approach and whole-core

\section{Completeness of Modeling Package:}

There are sufficient options available for the user to make intelligent choices from a number of state-of-the art options, with the facility for a range of sensitivity evaluations.

\section{Package Numerics:}

Tabular/interpolation methods are used in this package. 


\section{MODEL TITLE: DECAY HEAT POWER}

\section{MELCOR Package Identifier: DCH}

\section{Model Description and Pedigree:}

The package provides modeling of the heating resulting from the radioactive decay of fission products.

In the radionuclide option, ORIGEN calculations are used as the basis for a model that tracks the decay power of a number of elemental groups. Inventory masses are based on ORIGEN results or can be input by the user.

Whole-core options are available (summation of decay heat data from ORIGEN calculations, ANS-5.1-1979 standard, user-defined by a control or tabular function).

In the radionuclide option, daughter isotopes are assumed to be transported along with their parents. A total of 29 elemental groups are divided among 13 different chemical classes. A single table of normalized decay power after shutdown is used for both PWRs and BWRs.

The modeling is described in Refs. K-5 through K-7, with additional material in Ref.K-8. A recent assessment carried out at LANL is described in Refs. K-9 and K-10.

\section{Implementation Within MELCOR:}

DCH provides decay heat data to various packages as required as radioactive core materials relocate. Logarithmic interpolation in time is used to determine the power for each elemental group from tables.

No inconsistencies in the implementation have been found.

Comprehensive options are available for user-defined input of decay power, mass inventories, group classification, etc., through the MELGEN package.

\section{Results of Model and Parameter Sensitivity Studies:}

ORIGEN and ANS-5.1-1979 standalone results have been compared (Ref. K-11), the comparison showed some inconsistency (Ref. K-12).

\section{Results of Model Benchmarking/Validation Activities:}

No specific items have been identified.

\section{Identified Deficiencies and Planned Improvements:}

There is some need for clarification and documentation on the way decay heats are normalized and on the clarification of plot variables. Package identifiers can be confused with direct containment heating (Ref, K-13). There are no specific plans to deal with these minor deficiencies.

\section{Summary Findings:}

The package provides a choice of physically reasonable treatments of decay heat generation. The methods can be regarded as state-of-the-art. There is considerable scope for users to tailor the calculation in critical cases. 


\section{Importance Assessment:}

Decay heat is a major heat source and is a dominant phenomenon. However, the treatment is good and the contribution to the uncertainty in the source term calculation is judged to be small.

\section{Technical Adequacy:}

Category 1. 


\section{MODEL TITLE: MATERIALS PROPERTIES}

\section{MELCOR Package Identifier: MP}

\section{Package Modeling Concepts:}

This package provides material property data as requested by other packages such as the COR and HS modules.

\section{Relationship to Dominant Phenomena:}

The package is relevant to the initial transient, melt, relocation, slump, eutectic behavior, control rod/blade behavior, and refreezing.

Package Governing Equations and Numerics:

There is extensive use of of tabular data and interpolation techniques.

\section{Completeness of Modeling Package:}

A few properties are absent and the origin of some data is unknown. Further information is given in the detailed review of this package. 


\title{
MODEL TITLE: MATERIALS PROPERTIES
}

\author{
MELCOR Package Identifier: MP
}

\section{Model Description and Pedigree:}

The MP package supplies material property data mainly to the HS and COR packages. Some other discrete packages, such as CORCON, use their own libraries. Specific heat, melting temperature, heat of fusion, thermal conductivity, and density are evaluated for Zircalloy, Zircalloy oxide, steel, steel oxide, boron carbide, $\mathrm{Ag} / \mathrm{In} / \mathrm{Cd}$ alloy, uraniım, uranium dioxide, graphite, and concrete. Thermal conductivities of fluids such as water/steam, air, and hydrogen are also calculated, as well as the density of steam and air. Binary diffusivities of fluids can also be calculated. materials.

A wide variety of empirical correlations is used. There is provision for user-defined

The package is described in Refs. K-5 and K-6, with additional material in Ref. K-8.

\section{Implementation Within MELCOR:}

Properties for user-defined materials may be specified (in MELGEN). There is no check on the consistency of user-defined enthalpy, specific heat capacity, melting temperature, and latent heat of fusion; any inconsistency here may give run-time errors that may be difficult to trace.

On numerical methods, there is extensive use of tabular data.

\section{Results of Model and Parameter Sensitivity Studies:}

Model/parameter sensitivity studies are inappropriate for this package.

\section{Results of Model Benchmarking/Validation Activities:}

No direct testing of the MP package has been identified. Some indirect validation by comparing heatup data from melt progression experiments, e.g., PBF SFD 1.1 and 1.4 (Refs, K-1 and $\mathrm{K}-2$ ) and the Phebus ISP-28 analysis (Ref. K-3), shows no serious errors.

\section{Identified Deficiencies and Planned Improvements:}

The lack of consistency checking of thermal properties for user-specified material has been mentioned above. There are a few gaps in the table of physical property vs material type.

The sources of many of the data are unknown (although some of these data are consistent with established literature references).

In Version 1.8.0 of the code, boron carbide properties were used for $\mathrm{Ag} / \mathrm{ln} / \mathrm{Cd}$ alloy as well (replaced by suitable data from MELPROG in a later release of the code, as seen in Ref. $K \cdot 14$, although this change is not fully documented).

Sensitivity coefficients are needed for some correlations (e.g., for binary diffusivities).

The calculation of transport properties of mixtures should be centralized in the MP package. Transport properties for a user-defined gas are assumed to be those of steam (poor approximation for helium, for example). This problem can be circumvented be re-defining an existing gas, for which procedure the user needs skill and knowledge.

There are no properties for eutectic mixtures. These will be required if eutectic models are introduced into the COR package. The current state of knowledge is fair here (e.g., reasonably well known for $\mathrm{U} / \mathrm{Zr} / \mathrm{O}$ mixtures), becoming poorer as an accident proceeds. The large number of compositions would make a comprehensive treatment difficult. 
There are inconsistencies through the code in material data, since some of the packages (CORCON being a prime example) use their own formulations. The transfer process package (TP), which handles the transfer of mass and energy among the COR, FDI, CAV and RN packages, is assessed separately.

Several deficiencies within the MP package are recognized by Sandla, and a work program has been proposed to remedy them (Refs. K-13 and K-15). Substantial progress has been reported in the area of calculating thermal conductivities and viscosities of gas mixtures (using standard semi-empirical formulae, Ref, $\mathrm{K}-4$ ) and these improvements are included in Version 1.8 .1 of the conde.

\section{Summary Findings:}

There is a need for a considerable amount of review and tidying up in the MP package; the sources of some data need identifying, inconsistencies should be removed and documentation brought up to date. The MATPRO compilation would be a good baseline. Where references are cited, the choices are reasonable, though not necessarily the most recent. The adjudged technical adequacy level reflects mainly uncertainty in properties of mixtures; properties for unreacted core materials (e.g., $\mathrm{UO}_{2}$, Zirconium) are fully sufficient.

\section{Importance Assessment:}

The thermophysical properties of the major core constituents need to be well modeled. Uncertainties in the important properties are probably small, but more reassurance is needed. This package is unlikely to be a major contributor to the uncertainty in the calculation of the source tern.

\section{Technical Adequacy:}

Category 2.

Eutectics, AM: Category 6.

PRA: Category 7. 


\section{MODEL TITLE: NONCONDENSABLE GAS EQUATION OF STATE}

\section{MELCOR Package Identiffer: NCG}

\section{Model Description and Pedigree:}

Noncondensable gases are modeled as general ideal gases, with temperature-dependent specific heats. Basic data are from JANAF and other sources. Heats of formation are included in the enthalpy functions, using a thermochemical reference point so that heats of reaction are accounted for naturally.

Gases for which properties are held in an internal library are hydrogen, oxygen, carbon dioxide, carbon monoxide, nitrogen, nitrogen monoxide, nitrous oxide, ammonia, acetylene, methane, and ethylene (hellum not included). User-defined gases may also be specified. $\mathrm{K}-8$

The modeling is described in Refs. K-5 and K-6, with some additional material in Ref,

\section{Implementation Within MELCOR:}

The package is called from the CVT package. The consistency is reasonable for thermodynamic quantities.

There is provision for input of properties of user-defined gases, changes to coefficients of library gases (in MELGEN package), and a sensitivity coefficient for the natural temperature in the integral of specific heat.

The numerical methods involve analytic fits to temperature-dependent properties.

\section{Results of Model and Parameter Sensitivity Studies:}

No model or parameter sensitivity studies have been identified.

\section{Results of Model Benchmarking/Validation Activities:}

No model benchmarking or validation studies have been identified.

\section{Identified Deficiencies and Planned Improvements:}

The origin of many of the quantities in the library of pre-set data is unknown (code Version 1.8.0). This is recognized by SNL.

A revision of these data, with improved documentation, has been proposed by the SNL team (Ref. K-8). However, these changes are not included in the version of the code under review.

\section{Summary Findings:}

The methods are reasonable and adequate, but the data base is ill-defined in some areas. This deficiency is recognized and resolution is proposed by SNL, but not as a top priority item. This review accepts that there are more important deficiencies elsewhere. 


\section{Importance Assessment:}

The topic is of medium importance. The proposed changts should remove the identified deficiencies, when they are implemented. The overall contribution to uncertainty in the source term is, however, likely to be small.

\section{Technical Adequacy:}

Category 1. 


\section{MODEL TITLE: TRANSFER PROCESS}

\section{MELCOR Package Identifier: TP}

\section{Package Modeling Concepts:}

The transfer package provides a standard interface for modeling packages such as COR, FDI, CAV, and RN to transfer mass and energy among themselves. The properties are transferred in and out of a centrally maintained data base.

\section{Relationship to Dominant Phenomena:}

The package contains no physical modeling; therefore the findings are inappropriate under this heading.

\section{Package governing equations and numerics:}

This package uses a matrix approach, with the transfer processes currently being specified by the user.

\section{Completeness of Modeling Package:}

The package is judged to carry out all necessary functions. 


\section{MODEL TITLE: TRANSFER PROCESS}

\section{MELCOR Package Identifier: TP}

\section{Model Description and Pedigree:}

The transfer package provides a facility for transferring mass and energy among the COR, FDI, CAV, and RN modules. The data are stored in a central data base; data are moved into or out of this data base as required. The relationships between the "in" and "out" transfer processes are defined by the user. Masses may change identity between packages, e.g., steel might be referenced in one package while its constituent elements might be referenced in another. Total enthalpy may not be conserved if there are differences in equations of state between the packages involved in a given transfer.

The package is described in Refs. K-5 and K-6.

\section{Implementation Within MELCOR:}

The user is required to specify the translation matrices among packages. However, a number of defaults are available for use (given in the user's guide, Ref. K-5). Creation or retention of mass in any transfer is noted in the code output, along with any enthalpy alteration.

\section{Results of Model and Parameter Sensitivity Studies:}

Sensitivity studies are inappropriate for this package.

\section{Results of Model Benchmarking/Validation Activities:}

Benchmarking and validation are inappropriate for this package. No reporting on testing of this package has been identified.

\section{Identified Deficiencies and Planned Improvements:}

It is intended that much of the transfer of mass and energy data will be made transparent to the user, but no time scale for this code enhancement has been identified. At present, there is much reliance on the user providing sensible input.

\section{Summary Findings:}

The methods are reasonable and adequate, given the problems involved in transferring mass and energy data among packages that do not necessarily have consistent equations of state. It would be preferable if such inconsistencies were eliminated, but this is judged to be unnecessary in the shon term.

\section{Importance Assessment:} the code.

The accurate transfer of mass and energy data is important for the reliable functioning of 


\section{Technical Adequacy:}

This package cannot be formally assigned a category within the current system, since no modeling of phenomena is involved. However, the treatment is judged to be as reasonable as could be expected, given the problems involved. 


\section{MODEL TITLE: WATER PROPERTIES}

\section{MELCOR Package Identifier: H20}

\section{Model Description and Pedigree:}

The package calculates thermodynamic water properties based on an analytic formulation of the Keenan and Keyes equation of state extended at temperatures greater than $1589 \mathrm{~K}$ using JANAF data. derivatives.

Single-phase properties are evaluated using the Helmholtz function and its term-by-term

Mixed-phase properties are evaluated using tables for each phase $\left(1^{\circ} \mathrm{C}\right.$ intervals) calculated external to MELCOR. The coexistence curve is defined by points where pressure, temperature, and the Gibbs function are equal for two different values of density. Two-phase properties are evaluated from the tables using the lever rule.

The modeling is limited to temperatures above $0^{\circ} \mathrm{C}$ and pressures of less than $100 \mathrm{MPa}$ (reasonable for plant studies).

The modeling is described in Refs. K-5 and K-6.

\section{Implementation Within MELCOR:}

The package is called from the CVT package. The analytic formulation avoids discontinuities and inconsistencies in properties. The Keenan and Keyes formulation is consistent with the JANAF data at their common boundary $(1589 \mathrm{~K})$.

There is no user input to this package.

\section{Results of Model and Parameter Sensitivity Studies:}

Model and parameter sensitivity studies are not appropriate for this package.

\section{Results of Model Benchmarking/Validation Activities:}

Some unpublished checking has been carried out, but this is not available for review.

\section{Identified Deficiencies and Planned Improvements:}

No deficiencies have been identified and, therefore, no improvements are planned.

\section{Summary Findings:} and data.

This is a consistent, state-of-the-art package using well-established, reasonable methods

\section{Importance Assessment:}

It is highly important to calculate steam/water properties consistently and accurately. The $\mathrm{H} 20$ package meets these requirements. Contribution to source term uncertainty is judged to be small. 


\section{Technical Adequacy:}

Category 1.

\section{REFERENCES}

K-1. I. R. Madni, "MELCOR Sitimulation of the PBF Severe Fuel Damage Test 1-1," in Proceedings of the 26th National Heat Transfer Conference, AIChE Symposium Series on Heat Transfer, Philadelphia, Pennsylvania (1989).

K-2. I. K. Madni, "MELCOR 'Lessons Learned': A User Perspective," Brcokhaven National Laboratory draft letter report to R. Foulds, US Nuclear Regulatory Commission (January 9, 1991).

K-3. C. C. Wong, "A Standard Problem for HECTR-MAAP Comparison: "ncomplete Burning," Sandia National Laboratories report SAND87-1858 (NUREC CR-4993) (August 1988).

K-4. B. E. Boyack, Los Alamos National Laboratory, unpublished questions on "HS and COR Packages," by R. Viskanta, in letter to B. E. Boyack (December 27, 1990). Unpublished answer on "Responses to Additional Viskanta Questions on HS Package Modeling" by R. M. Summers, Sandia National Laboratories, Third MELCOR Peer Review Meeting, January 11, 1991. Unpublished answer on "Responses to Additional Viskanta Questions on COR Package Modeling," by R. M. Summers, Sandia National Laboratories, Third MELCOR Peer Review Meeting, January 11, 1991.

K-5 . R. M. Summers, "MELCOR Primer, Version 1.8.0," Sandia National Laboratories document (February 17, 1989). Primer includes the following sections:

MELCOR Architecture Programmer's Guide

Machine Dependent Code in MELCOR

MELCOR/MELGEN

Users' Guides

Burn Package

Cavity Package

Control Function Package

Core (COR) Package

Control Volume Hydrodynamics Package

Decay Heat Package

Fan Cooler (ESF/FCL) Package

External Data File Package

FDI Package

Flow Path

IS Package

Material Properties

MELPLT Users' Guide

Noncondensible Gas EOS

Radionuclide Package

Containment Sprays Package

Tabular Function

Transfer Process

Reference Manuals

Durn Package 
Core (COR) Package

Thermal Hydraulic Packages (CVH and FL)

Control Volume Thermodynamics Package (CVT)

Heat Structure (HS) Package

Radionuclide Package

Fan Cooler (ESF/FCL) Package

K-6. R. M. Summers, R. K. Cole, Jr., E. A. Boucheron, M. K. Carmel, S. E. Dingman, and J. E. Kelly, "MELCOR 1.8.0: A Computer Code for Severe Nuclear Reactor Accident Source Term and Risk Assessment Analyses," Sandia National Laboratories SAND9()0364 (NUREG/CR-5531) (January 1991).

K-7. R. M. Ostmeyer, "An Approach to Treating Radionuclide Decay Heating For Use in the MELCOR Code System," Sandia National Laboratories report SAND84-1404 (NUREG/CR-4169) (May 1985).

K-8. T. Haste, "Summary of Discussions on Decay Heat and Material Properties on November 8, 1990," Thermal Reactor Services, AEA Technology, Winfrith Technology Center summary report (December 18, 1990).

K-9. J. S. Elson, "MELCOR Peer Review: MELCOR Decay Heat Package," Los Alamos National Laboratory Group N-12 memorandum N-12-90-609 to B. E. Boyack (October 16, 1990).

K110. J. S. Elson, "MELCOR Decay Heat Package-Addendum," Los Alamos National Laboratory Group N-12 memorandum N-12-90-721 to B. E. Boyack (November 28, 1990).

K.11. F. Eric Haskin, "Whole-Core Decay Heat Power," Sandia National Laboratories memorandum (January 28, 1986).

K-12. W. T. Pratt, R. Denning, T. S. Kress, D. Powers, J. Gieseke, M. Khatib-Rahbar, and K. R. Perkins, "Evaluation of MELCOR as a Source Term Analysis Code," Brookhaven National Laboratory draft report (September 1), 1985).

K-13. R. M. Summers, "MELCOR 1.8.0 Shortcomings," Sandia National Laboratories draft document (January 10, 1990).

K.14. B. E. Boyack, Los Alamos National Laboratory, unpublished questions on "Core Modeling" and "Material Properties," by T. J. Haste, in letter to B. E. Boyack, Regarding: MELCOR Peer Review-Second Meeting, September 27, 199(). Unpublished answers on "Answers to Tim Haste's Questions on COR Package Modeling" by R. M. Summers, Sandia National Laboratories, Second MELCOR Peer Review Meeting, November 5-7, 1990. Unpublished answers on "Responses to Additional Haste Questions on COR Package Modeling," by R. M. Summers, Third MELCOR Peer Review Meeting, January 11, 1991.

K-15. R. M. Summers, R. K. Cole, Jr., and E. A. Boucheron, "MELCOR Improvement Needs Assessment," Sandia National Laboratories letter report to R. B. Foulds, US Nuclear Regulatory Commission (October 17, 1990). 


\section{APPENDIX L,}

\section{(Other Models Missing From MELCOR}

The following list of phenomena are not modeled in MELCOR. Their omission was judged to be less critical than those listed in Chapter 3. Nevertheless, they are listed here for completeness.

1. Melting of heat structures represented in the HS package, i.e. all those not represented by COR (see Appendix E).

2. Detonations of hydrogen or carbon monoxide (see Appendix I).

3. Dissociation of steam at high temperatures (see Appendix F).

4. Chemical reactions at metal surfaces undergoing film boiling (see Appendix E).

5. Plugging of gas flowpaths by settled aerosols (this can probably be modeled using control functions) (see Appendix G).

6. Resuspension of settled aerosols by high gas velocities (see Appendix $\mathrm{G}$ ).

7. Transport of liquid films created by aerosol settling, i.e., drainage under gravity or drag by high gas flows (see Appendix G).

8. Conduction of heat into the concrete underneath a debris pool when ablation is not occurring (see Appendix $\mathrm{H}$ ).

9. Nonablative thermal interaction of debris and concrete (see Appendix $\mathrm{H}$ ). 

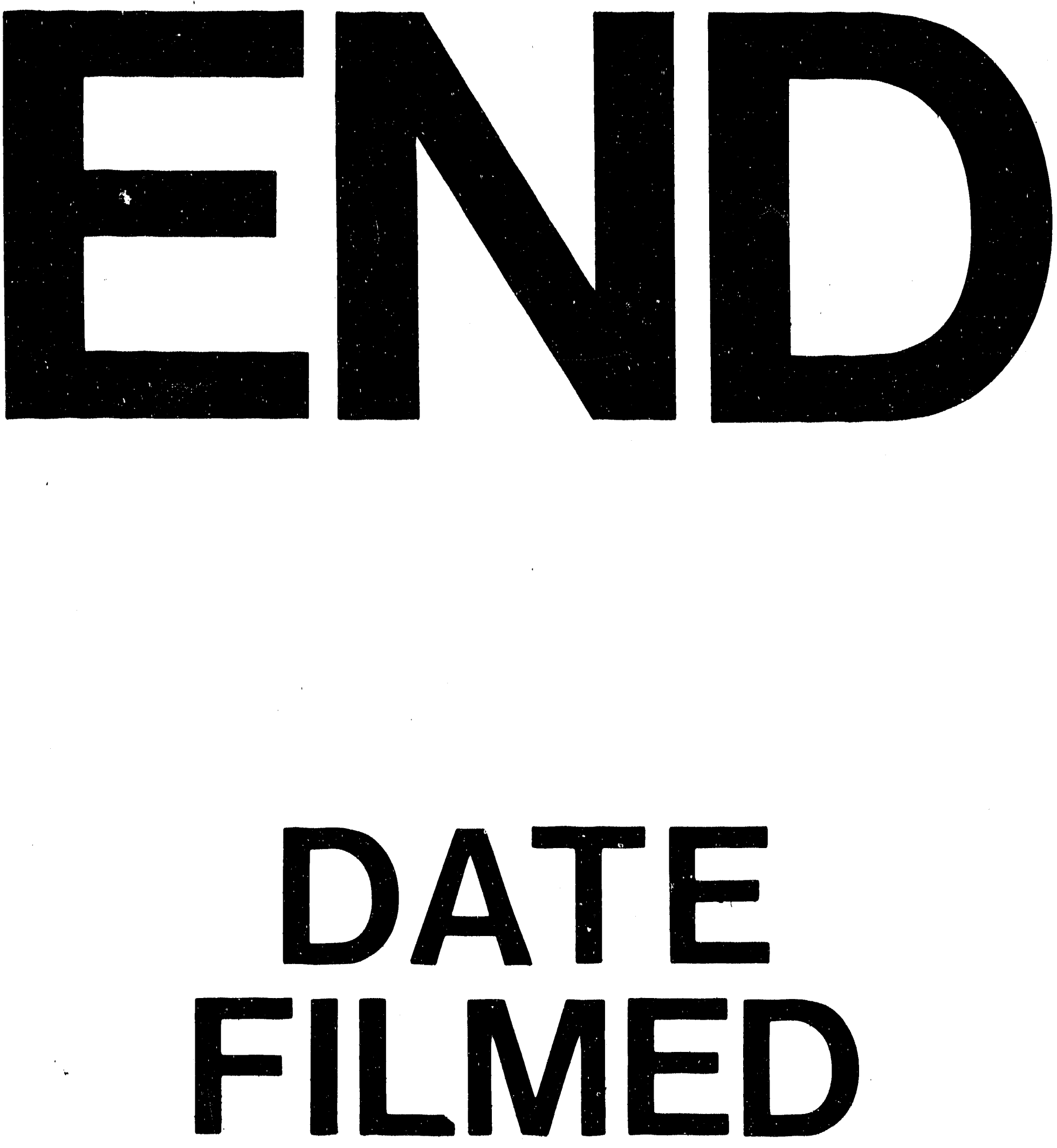

1

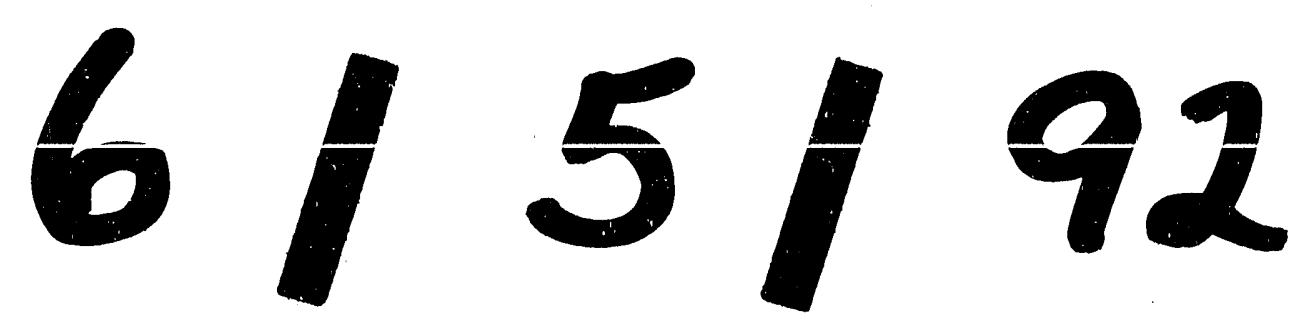


